\title{
ANNA SENNEBY \\ RE-EVALUATION OF \\ METHODS TO IDENTIFY \\ INDIVIDUALS WITH \\ INCREASED RISK OF CARIES
}
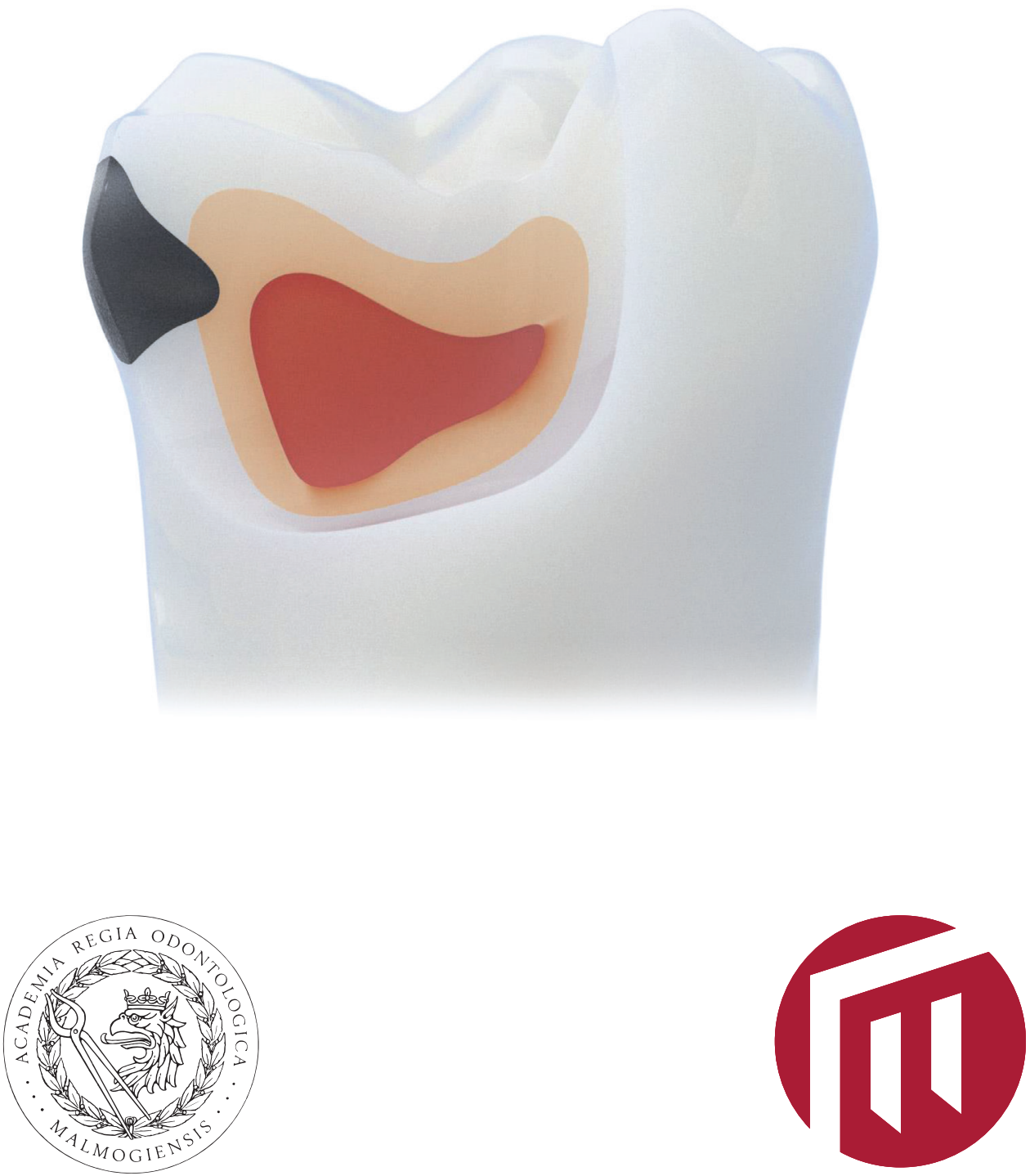

MALMÖ UNIVERSITY 

RE-EVALUATION OF METHODS TO IDENTIFY INDIVIDUALS WITH INCREASED RISK OF CARIES 
Malmö University, Faculty of Odontology Doctoral Dissertation 2017

(C) Anna Senneby, 20I7

Cover illustration: Johan Birger

ISBN 978-9I-7I04-722-9 (print)

ISBN 978-9I-7I04-723-6 (pdf)

Holmbergs, Malmö 2017 


\section{ANNA SENNEBY \\ RE-EVALUATION OF METHODS TO IDENTIFY INDIVIDUALS WITH INCREASED RISK OF CARIES}

Malmö University, 2017

Faculty of Odontology Malmö, Sweden 
This publication is also available in electronic format at: www.mah.se/muep 
To my family 



\section{CONTENTS}

LIST OF ARTICLES ........................................... 10

ABSTRACT ................................................. 11

POPULÄRVETENSKAPLIG SAMMANFATTNING

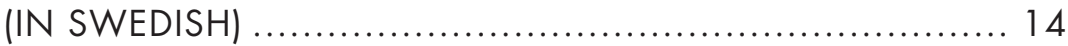

ABBREVIATIONS AND DEFINITIONS ....................... 16

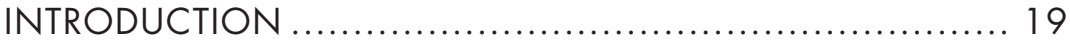

Oral Health and Diseases................................................. 19

Coronal Dental Caries ..................................................... 19

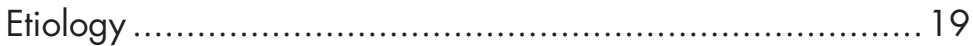

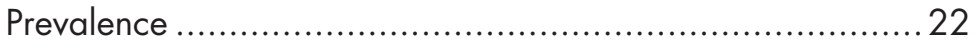

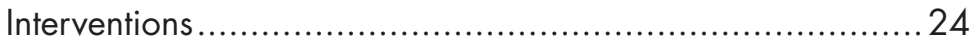

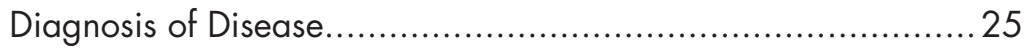

Quantification of Probabilities and Uncertainties.................26

Validity, Reliability and Agreement.................................29

Caries Lesion Detection ..................................................... 30

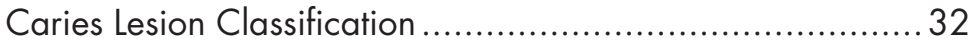

Caries Lesion Progression ................................................. 33

Caries Risk Assessment Methods .......................................... 34

OBJECTIVES ................................................ 36

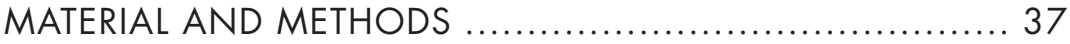

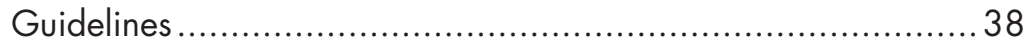

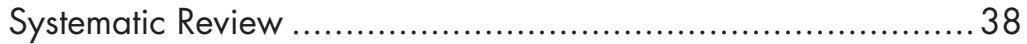

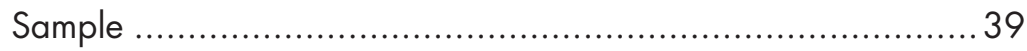


Clinical, Radiographic and Microbiological Examinations .........40 40

Evaluation of Diagnostic Methods ....................................... 43

Previous Caries Experience ............................................ 44

Dentists' Risk Assessments ............................................. 44

Acid Tolerance of Dental Biofilm ..................................... 44

Reference Standard - Caries Lesion Progression ..................... 45

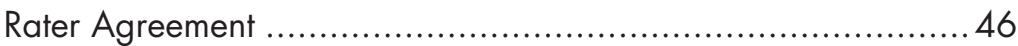

Radiological Assessment of Approximal Caries Lesions and Caries Lesion Progression ........................................ 46

Assessment of Proportion Acid Tolerant Cells ..................... 47

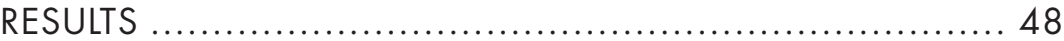

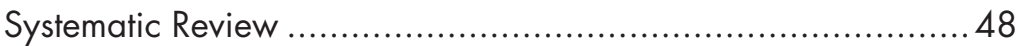

Assessment of Risk of Bias............................................49 49

Sample Characteristics Concerning Caries Status ....................50

Acid Tolerance in Dental Biofilm ..........................................50

Validity of the Interval Scale and Consistency

of Acid Tolerance Assessment Method .................................52

Diagnostic Accuracy of Methods to Identify Individuals with Increased Risk of Caries Development ...........................552

Previous Caries Experience .............................................55

Dentists'Risk Assessments ...............................................56

Acid Tolerance of Dental Biofilm .....................................56

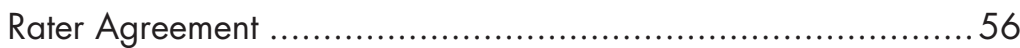

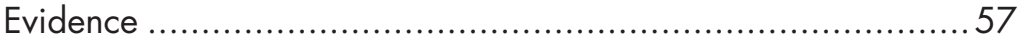

DISCUSSION............................................... 60

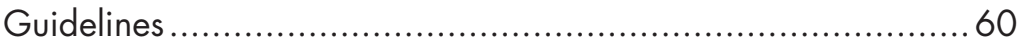

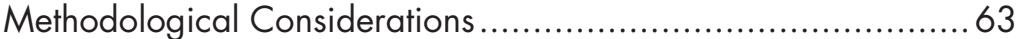

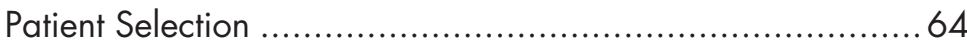

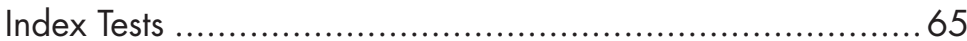

Reference Standard - The Not So Golden Standard .............68 68

Flow and Timing ......................................................... 70

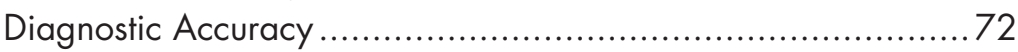

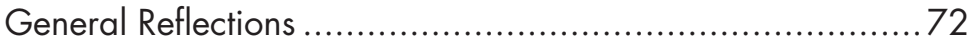

Some Factors Influencing Accuracy Estimates ....................73

Previous Caries Experience .......................................... 75

Dentists' Risk Assessments ..............................................76

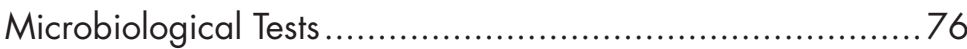

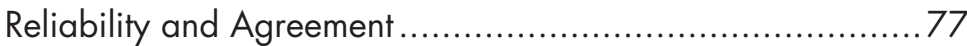


Risk .78

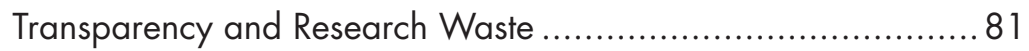

Evidence - or Rather the Absence of Evidence ........................ 82

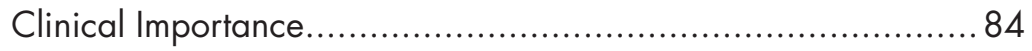

CONCLUSIONS ........................................... 85

FUTURE RESEARCH....................................... 87

ACKNOWLEDGEMENTS .................................. 89

REFERENCES ............................................... 92

PAPERS I - IV ............................................. 107 


\section{LIST OF ARTICLES}

This thesis is based on the following articles, referred to in the text by their Roman numerals. All articles are reprinted with the permission from the copyright holders and appended to the end of the thesis.

I. Diagnostic accuracy of different caries risk assessment methods. A systematic review. Senneby A, Mejàre I, Sahlin NE, Svensäter G, Rohlin M. J Dent. 2015;43(12):1385-93. doi: 10.1016/j. jdent.2015.10.011.

II. A novel classification system for assessment of approximal caries lesion progression in bitewing radiographs. Senneby A, Elfvin M, Stebring-Franzon C, Rohlin M. Dentomaxillofac Radiol. 2016;45(5):2016. doi: 10.1259/dmfr.20160039.

III. Acid tolerance properties of dental biofilms in vivo. Senneby A, Davies JR, Svensäter G, Neilands J. Submitted to BMC Microbiol. 2017.

IV. Threshold Values Affect Diagnostic Accuracy of Caries Risk Assessment Methods. Senneby A, Neilands J, Svensäter G, Axtelius B, Rohlin M. To be submitted to Acta Odontol Scand. 


\section{ABSTRACT}

The overall aim of this thesis was to re-evaluate methods used to identify individuals with increased risk of coronal caries lesion development. The specific aims were to:

- evaluate diagnostic accuracy of different methods used to identify individuals with increased risk of developing coronal caries lesions (study I)

- design and pilot a novel classification system for the assessment of caries lesion progression in bitewing radiography and to report rater agreement of the system (study II)

- assess intra-individual differences in biofilm acid-tolerance between different tooth surfaces and inter-individual variation as well as stability of acid tolerance over time (study III)

- estimate threshold effects on diagnostic accuracy of (i) previous caries experience, (ii) dentists' risk assessments and (iii) acid tolerance of dental biofilm to identify adolescents with increased risk of developing coronal caries lesions (study IV). 
Study I comprised a systematic review of caries risk assessment methods. Following methods were included for final analysis: previous caries experience, tests using microbiota, buffering capacity, salivary flow rate, dental plaque, dietary habits, and sociodemographic variables. The included studies were assessed for risk of bias with the aid of QUADAS-2, which resulted in identification of methodological limitations concerning study design, test technology and reporting. There was low evidence for methods using previous caries experience and salivary mutans streptococci and there was no evidence regarding other methods. The overall evidence concerning the validity of analysed methods used for caries risk assessment is limited. As methodological quality was low, there is a need to improve study design. Furthermore, candidate biomarkers for caries risk assessment was called for.

In study II a classification system with drawings and text was designed to assess caries lesion progression in bitewing radiographs. Guidelines for Reporting Reliability and Agreement Studies, GRRAS were used to design and report the study. Pairs of posterior bitewing radiographs (baseline and 1-year follow-up) of teeth with different status concerning caries lesion progression were selected and 10 raters assessed the radiographs with the aid of the classification system. Seven raters repeated their assessments.

Kappa for the interrater agreement of 10 raters assessing progression was 0.61 , indicating substantial agreement. Corresponding intrarater agreement was substantial (kappa 0.66-0.82). This classification system can provide a common framework for clinical decisionmaking on caries interventional methods and patient visiting intervals. Scientifically, the system allows for a comparative analysis of different methods of prevention and treatment of caries as well as of different caries risk assessment methods.

In study III dental biofilms were collected from supragingival approximal surfaces in adolescents at baseline and after 3 days as well as after 1, 6 and 12 months. After exposure to $\mathrm{pH} 3.5$ for 2 hours, biofilm bacteria were visualized with LiveDead ${ }^{\circledR}$ BacLight $^{\mathrm{TM}}$ staining and vitality examined using an inverted confocal scanning laser microscope. Bacteria stained green (live) were considered acid- 
tolerant and red bacteria (damaged) not acid-tolerant. Images of the biofilm were assessed using a scale (score 1-5) to determine the proportion acid-tolerant bacteria in each sample.

A majority of the adolescents showed low scores of biofilm acid tolerance. In 14 of 20 individuals, no differences were seen between three dental sites of the same individual. At 51 of total 60 sites, scores for acid tolerance were unchanged over a 3-day period. However, scores for acid tolerance changed over a 1 -year period in $50 \%$ of the individuals. Thus, acid tolerance of dental biofilm seems to be a candidate method that may be implemented in caries risk assessment after clinical prospective studies have been performed.

For the purpose of study IV, the STARD-statement "Standards for Reporting of Diagnostic Accuracy" was implemented for design and reporting of the study. Adolescents, aged 12, visiting two public dental clinics were examined by visual/tactile examination and bitewing radiography of posterior teeth at baseline and after 1 year. Two established methods (previous caries experience and dentists' risk assessments) and one candidate method (acid tolerance in dental biofilm) were applied with pre-specified thresholds. The reference standard comprised coronal caries lesion development after 1 year. Caries lesion progression occurred in $29 \%$ of the adolescents, mainly as approximal lesions within enamel.

Diagnostic test accuracy varied with thresholds of the index tests, of the reference standard and with different caries status and caries lesion progression. Most positive likelihood ratios for the methods were $<5$ indicating small increases in the post-test probabilities. Therefore, it may difficult to design a universal model for caries risk assessment. 


\section{POPULÄRVETENSKAPLIG SAMMANFATTNING (IN SWEDISH)}

Med en tillförlitlig riskbedömningsmetod kan man säkrare förutspå vem som får kariesskador i framtiden. Då ökar chansen att man sätter in förebyggande åtgärder till de patienter som har nytta av åtgärderna. Det medför också att besöksintervallet hos tandvården bättre kan anpassas utifrån patientens behov. De riskbedömningsmetoder som används inom tandvården idag, och som återfinns i vetenskaplig litteratur, är inte tillförlitliga. Resultatet blir att patienter med ökad risk för kariesutveckling kan missbedömas. De övergripande målen med denna avhandling var att grundligt utvärdera befintliga riskbedömningsmetoder för kariesutveckling samt skapa en plattform för nya bättre metoder.

När man utvärderar om en riskbedömningsmetod är tillförlitlig behöver man bedöma kariesutvecklingen hos de patienterna som genomgått metoden. En grundläggande förutsättning för detta är att bedömningen av kariesutvecklingen dvs. kariesprogressionen i en röntgenbild är adekvat. Därför har överensstämmelsen mellan olika tandläkare, som använt sig av en ny mall för att bedöma kariesprogression i röntgenbilder, analyserats. Tandläkarnas bedömningar stämde väl överens både mellan tandläkarna och när en tandläkare upprepade sin bedömning. 
En ny metod för riskbedömning har utvärderats i avhandlingen. I korthet innebär den att man bedömer proportionen syratoleranta bakterier i placket på tandytor. En hög andel syratoleranta bakterier är rimligen en förutsättning för att en kariesskada ska utvecklas. Syratoleranta bakterier favoriseras av en sur plackmiljö på tandytan, medan andra bakterier inte klarar sig $i$ en sådan miljö. Resultaten $i$ denna avhandling ger vissa belägg för att detta kan vara en användbar metod, men metoden behöver vidareutvecklas och utvärderas i fler och större vetenskapliga studier. Två andra metoder har också utvärderats i avhandlingen. Dessa bygger på att man bedömer patientens tidigare karieserfarenhet, dvs. om patienten har eller har haft kariesskador på tänderna. Metodernas tillförlitlighet varierar beroende på olika faktorer, till exempel sjukdomsförekomst och sjukdomspanorama (fördelning av svår respektive lindrig sjukdom) hos patienterna. Detta innebär att en metod, som är bra för en grupp patienter inte nödvändigtvis presterar lika bra om den används i en annan grupp av patienter.

Således är de vetenskapliga bevisen, "evidensen”, för riskbedömning av kariesutveckling hos patienter inte tillräckliga för att avgöra om metoderna fungerar på ett tillförlitligt sätt. Framtida studier behöver vara tydligare i sin beskrivning av de metoder som använts och hur de har använts för att man ska kunna avgöra i vilket sammanhang de har utvärderats och kan användas. 


\section{ABBREVIATIONS AND DEFINITIONS}

\begin{tabular}{|c|c|}
\hline Agreement & $\begin{array}{l}\text { Agreement is defined as the degree to which } \\
\text { scores or ratings are identical i.e. how close the } \\
\text { results of the repeated measurements are, by } \\
\text { estimating the measurement error in repeated } \\
\text { measurements (Kottner et al., } 2011 \text { 1). The } \\
\text { term includes reliability and reproducibility of } \\
\text { methods as well }\end{array}$ \\
\hline Caries increment & $\begin{array}{l}\text { Number of new caries lesions between two } \\
\text { points in time }\end{array}$ \\
\hline $\begin{array}{l}\text { Caries lesion development } \\
\text { (synonym: caries lesion } \\
\text { progression) }\end{array}$ & $\begin{array}{l}\text { Development of a caries lesion from sound } \\
\text { tooth surface to detectable lesion in enamel } \\
\text { or dentin or from detectable to more extensive } \\
\text { lesion }\end{array}$ \\
\hline $\begin{array}{l}\text { Caries risk assessment } \\
\text { (synonym: caries } \\
\text { prediction) }\end{array}$ & $\begin{array}{l}\text { Methods/tests used to identify individuals with } \\
\text { increased risk of developing coronal caries } \\
\text { lesions }\end{array}$ \\
\hline Diagnostic accuracy & $\begin{array}{l}\text { The capacity to discriminate among the } \\
\text { alternative states of the object under study } \\
\text { (Swets \& Pickett, 1982) }\end{array}$ \\
\hline Diagnostic test & $\begin{array}{l}\text { Diagnostic procedures, such as laboratory } \\
\text { tests and x-rays, routinely performed on all } \\
\text { individuals or specified categories of individuals } \\
\text { in a specified situation, e.g., patients being } \\
\text { admitted to the hospital (U.S. National Library } \\
\text { of Medicine, } 2017 \text { a) }\end{array}$ \\
\hline DMFS/DMFT & $\begin{array}{l}\text { Decayed Missing Filled Surfaces/ } \\
\text { Decayed Missing Filled Teeth } \\
\text { (permanent teeth) }\end{array}$ \\
\hline
\end{tabular}


ICDAS

International Caries Detection and Assessment System (Ismail et al., 2007)

\begin{tabular}{|c|c|}
\hline Index test & $\begin{array}{l}\text { Test/method under evaluation (Cohen et al., } \\
\text { 2016) }\end{array}$ \\
\hline Prevalence & $\begin{array}{l}\text { The proportion individuals exhibiting dental } \\
\text { caries lesions (in percentage) or the amount } \\
\text { of dental caries in an individual or a group of } \\
\text { individuals (DMFT/DMFS) (WHO, Oral health } \\
\text { database) }\end{array}$ \\
\hline $\begin{array}{l}\text { Rater } \\
\text { (synonym: observer) }\end{array}$ & $\begin{array}{l}\text { Every person who makes a judgement about a } \\
\text { person or object (Kottner et al., 2011) }\end{array}$ \\
\hline Reference standard & $\begin{array}{l}\text { The best available method for establishing the } \\
\text { presence or absence of the target condition } \\
\text { (Cohen et al., 2016) }\end{array}$ \\
\hline Risk & $\begin{array}{l}\text { The probability that an event will occur. It } \\
\text { encompasses a variety of measures of the } \\
\text { probability of a generally unfavourable } \\
\text { outcome (U.S. National Library of Medicine, } \\
2017 \mathrm{~b} \text { ) }\end{array}$ \\
\hline Risk of dental caries & $\begin{array}{l}\text { We considered coronal caries as an } \\
\text { unfavourable outcome for the individual. Risk } \\
\text { of dental caries was therefore defined as "The } \\
\text { development either (i) from sound tooth surface } \\
\text { to detectable lesion in enamel or dentin: i.e., } \\
\text { from health to disease or (ii) from detectable } \\
\text { to more extensive lesion: i.e., from disease to } \\
\text { more severe disease }\end{array}$ \\
\hline $\begin{array}{l}\text { Threshold value } \\
\text { (synonym: cut-off) }\end{array}$ & $\begin{array}{l}\text { A point dividing a population in subgroups } \\
\text { depending on the disease presence or severity }\end{array}$ \\
\hline
\end{tabular}





\section{INTRODUCTION}

The objective of this thesis was to develop, assess and validate methods to identify individuals with increased risk of coronal caries development.

Methods to identify individuals with increased risk of coronal caries lesion development are used daily in general dental practice and often form the basis for decisions concerning interventions and patients' visiting intervals. A prerequisite for increased effectiveness of preventive interventions is the accurate identification of target individuals i.e. those with an increased risk for future caries development and those not at risk for caries development (Mejàre et al., 2015). However, a knowledge gap exists regarding diagnostic accuracy of caries risk assessment methods.

\section{Oral Health and Diseases}

Dental caries is considered a serious public-health problem and oral diseases is the fourth most expensive disease to treat worldwide (WHO, 2007). Therefore, promotion of oral health is not only a cost-effective strategy, but also an integral part of health promotion in general, as oral health is a determinant of general health and quality of life. Dental caries experience in children of different ages is associated with a negative oral health-related quality of life (Do \& Spencer, 2007; Filstrup et al., 2003).

\section{Coronal Dental Caries}

\section{Etiology}

Throughout history, many theories on the aetiology of dental caries have been formed. These include tooth worms, parasites, disturbance 
of nutrition and many more (Miller, 1890). Already in the seventeenth century, Leeuwenhoek (1684) saw "little animals" in the scruff of teeth in his microscope, however for a long time his findings were doubted and even dismissed (Lane, 2015). In the twentieth century, the existence of Leeuwenhoek's' "little animals" was confirmed by the discovery of bacterial cells (Lane, 2015). Bacteria are one of the oldest known life forms on earth and definitely the most numerous. When mammals appeared on our planet bacteria had been established for billions of years (Amyes, 2014).

Today we believe that the human oral cavity exhibits a high degree of bacterial diversity with more than 700 taxa (Jenkinson, 2011). The oral microbiota is important to oral health as it prevents colonisation of pathogenic species and is thereby beneficial to the host (Marsh, 1994).

Numerous studies of the composition of microflora from sites with caries disease have been undertaken. As sites are normally colonised by complex microbial communities, the assessment of data presents a challenge. It is difficult to determine which species contribute to the development of disease, which ones have accumulated as a result of the disease, and which ones are merely innocent bystanders (Marsh, 2003).

The "non-specific plaque hypothesis" entered the field of caries aetiology in the 1960s and proposed that disease is a result of the overall interaction of all groups of bacteria in plaque and the amount of plaque. This theory is compromised by the fact that a limited subset of bacteria is consistently recovered in high numbers of diseased sites (Marsh, 2003).

For decades, Streptococcus mutans was considered the bacterial species principally responsible for the development of dental caries in humans (Keene \& Shklair, 1974; Tanzer et al., 2001) and most diagnostic, preventive, and therapeutic strategies have been targeted towards this microorganism (Simon-Soro \& Mira, 2015). Less frequently carious lesions were believed to be related to the activity of other streptococci or Lactobacillus spp (Loesche \& Straffon, 1979). This "specific plaque hypothesis" thus proposes that only a small proportion of the species in plaque cause caries (Loesche, 1976). However, the oral ecosystem is inhabited by hundreds of bacterial species, most of which are considered commensals, and species 
regarded as pathogens are frequently found in healthy individuals (Simon-Soro \& Mira, 2015). Caries can occur without mutans streptococci or mutans streptococci can be present without caries lesions developing (Marsh et al., 1989).

Since the mid-1980s, the "ecological plaque hypothesis" has prevailed. This hypothesis explains disease as a result of the characteristics of the microflora (phenotype) rather than as a result of which microorganism (genotype) that inhabits it (Marsh, 1994). Plaque-mediated disease is a consequence of a change in the local environment caused by enrichment of "oral pathogens" which disturbs the balance and thus the stability of the resident microflora (Marsh, 2003; Marsh, 1994; Marsh, 1991; Marsh \& Bradshaw, 1997).

Several bacteria, such as mutans streptococci and Actinomyces, is now considered to contribute to the demineralisation of tooth tissues (Beighton, 2005). Studies have shown that diseased sites are different from healthy ones in that they contain an increased amount of acidogenic and aciduric species, such as mutans streptococci (Streptococcus mutans and Streptococcus sobrinus) and lactobacilli, that contributes to demineralisation of enamel (Marsh, 2003).

As well as causing demineralisation, acids generated from carbohydrate metabolism by members of the biofilm affect the ecology of the biofilm itself. Support for a positive correlation between a flora with a higher acid-tolerance potential and lower plaque $\mathrm{pH}$ values has been found (Lingström et al., 2000). In the acidic environment created, these bacteria will have a competitive advantage and continue to flourish and achieve numerical dominance at the expense of more acid-sensitive species (Marsh, 2003). Consequently, an acid-tolerant microbiota will emerge promoting further change the oral ecosystem (Figure 1).

It has been shown that older biofilms have a higher proportion of acid-tolerant cells than newly formed biofilms (Welin-Neilands $\&$ Svensäter, 2007). However, little is known about the alteration over time of an acid tolerant biofilm in vivo. It is also not known if the biofilm acid tolerance is a site-specific phenomenon or if acid tolerance varies between different sites of the oral cavity. Furthermore, the proportion of acid tolerant bacteria in dental biofilm may be a candidate method to identify individuals at increased risk of caries lesion development. 
Societal level: Sociodemographic/economic variables

Individual level: Dietary variables, Oral hygiene measures, Salivary parameters
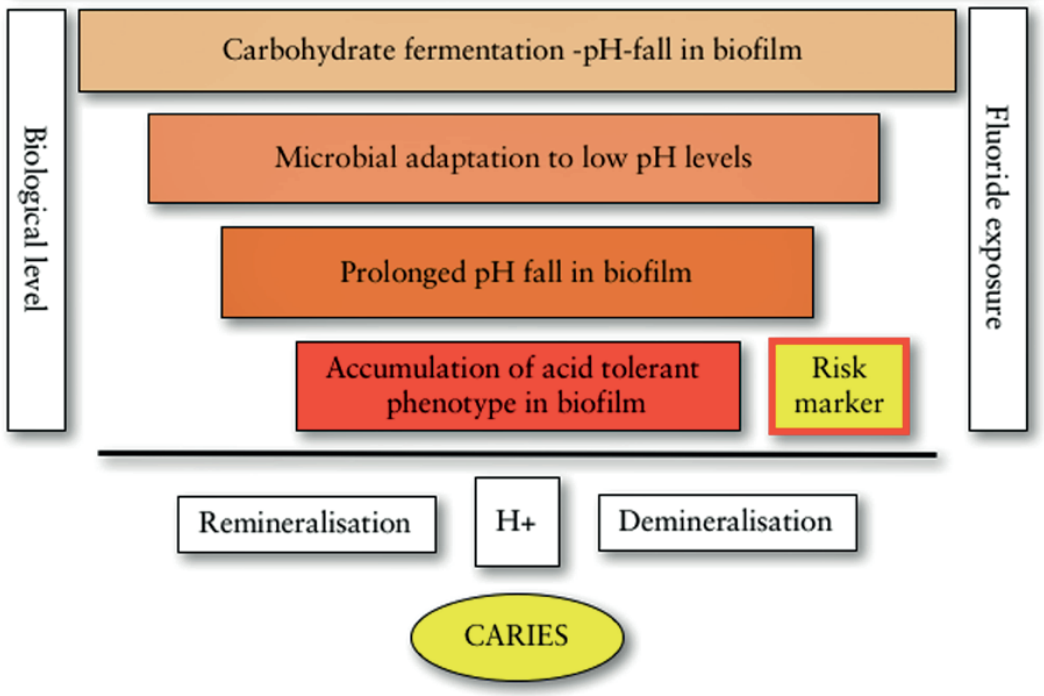

Figure 1. Factors on different levels influencing and contributing to caries lesion development. Original figure by Gunnel Svensäter, Malmö University.

\section{Prevalence}

Throughout history, humans, and indeed even the Neanderthals, have suffered from dental caries, albeit in low quantities (Topic et al., 2012). Iron Age remains found in Monte Bibele (Bologna) show a caries prevalence of about $10 \%$ (Gualandi, 1992). A similar frequency of carious teeth $(7 \%)$ was observed in remains from the Bronze Age found in Pakistan (Lukacs, 1992).

Teeth found in the Forum Romanum shows that some ancient Romans suffered from severe and prevalent caries (Fejeskov et al., 2012). This is comparable to contemporary populations in Africa and China without access to dental care (Luan et al., 2000; Manji et al., 1989). However, studies of skeletal remains from ancient Rome actually suggest that the caries prevalence among slaves and the middle-class was quite low, caries affected no more than $4-6 \%$ of the teeth found (Manzi et al., 1999). 
There was an increase in both caries lesions and severity from the middle Ages to the seventeenth century. This is believed to be connected to the dietary innovations of the seventeenth century, which included the consumption of cane sugar and refined flour (Moore \& Corbett, 1975). Prevalence of caries lesions continued to increase during the seventeenth century (Corbett \& Moore, 1976).

In Sweden, caries prevalence increased dramatically during the late 1800 s and the early 1900s. In the early 1960s, approximately one fourth of those over the age of 16 were completely edentulous due to caries or periodontal diseases, and nearly 100 per cent of all children and adolescents suffered from caries to a greater or lesser extent (SBU, 2002). This is in line with the first global map with data on DMFT for 12-year-olds during this period (1969), showing a high prevalence of caries in "industrialised countries" and lower values in "developing countries". Over the years, a change in caries prevalence with increasing levels of caries in certain "developing countries" and a decline in caries in many "industrialised countries" has occurred (WHO, 2017).

Thus, there have been improvements in the oral health of the populations of several countries, but in spite of this a global problem still persists. The problem is particularly noticeable among underprivileged groups in both developing and developed countries (Petersen, 2008). Trend of improving oral health has not been universal, it appears to have been reversed for some age groups in some of the countries in Eastern Europe during the period following the collapse of Communism (Künzel, 1996). This is especially the case in countries such as Bosnia and Herzegovina that have suffered wars (Ivankovic et al., 2003).

Major inequalities still exist, both within (The Swedish National Board of Health and Welfare, 2015) and between countries, in terms of disease severity and prevalence (Petersen et al., 2004). A decline in the prevalence and the severity of dental caries is particularly observed in countries with established public health programs using fluoride for dental caries prevention, coupled with changing living conditions, healthier lifestyles, and improved self-care practices (Petersen \& Ogawa, 2016).

In Sweden, the Swedish National Board of Health and Welfare (2017) states that the number of caries-free 19-year-olds has increased 
in recent years. Caries-lesion prevalence in Sweden is in accordance with WHO goals for 2020, lower than 1.5 DFT for 12-year-olds (0.70). In 2011, $65 \%$ of 12-year-olds were caries free which is an improvement with 2 units as compared to 2010 (The Swedish National Board of Health and Welfare, 2013).

However, according to Christensen et al. (2010) and Petersen (2008), there is also a striking difference between the social classes in the Nordic countries and a difference between regions is also evident (The Swedish National Board of Health and Welfare, 2017). The typically skewed distribution in caries prevalence seen in adolescents levels out during early adulthood (Mejàre et al., 2004).

\section{Interventions}

Fluoride enhances remineralisation of enamel, increasing mineral content of early carious lesions (Gao et al., 2016). To promote remineralisation, we need to detect early caries lesions (Ekstrand et al., 2001; Longbottom et al., 2009). Therefore, it's important to include bitewing radiography in the risk assessment enabling identification of individuals before cavity occur. There is a growing trend to delay operative intervention of caries lesions (Vidnes-Kopperud et al., 2011), emphasising early non-invasive intervention on the caries process (Pitts \& Wefel, 2009; SBU, 2002) instead of operative interventions. However, caries operative treatment thresholds, based on radiographic images, vary between dentists in different countries (Espelid et al., 1994; Gordan et al., 2009) and Scandinavian dentists predominantly restore surfaces that involve dentin (Espelid et al., 2001; Lith et al., 2002). In general dental health care, clinicians often have access to a series of radiographs of their patients taken over a period of several years. The decision concerning intervention of approximal caries can then be based not only on the clinical examination and the detection of caries lesions and lesion depth in the radiographs but also on the caries lesion progression rate.

Despite of extensive research undertakings, there are still uncertainties about evidence and the effect of non-surgical methods in relation to the prevention of caries (Axelsson et al., 2004; Mejàre et al., 2015; Tellez et al., 2013b) as well as the effectiveness of prevention in so-called "high risk" individuals (SBU, 2002). However, this does not mean that preventive methods are ineffective or that they should 
not be used (Axelsson et al., 2004). Several studies have found evidence for a caries-preventive effect including fluoride (Axelsson et al., 2004; SBU 2002; Tellez et al., 2013b). "Daily use of toothpaste with fluoride is an effective method of preventing caries in permanent teeth of children and adolescents (evidence grade 1)" (SBU, 2002). In locations where fluoride in toothpaste has been available for decades it is considered to be a main contributor to the decline in dental caries (Petersen \& Ogawa, 2016).

\section{Diagnosis of Disease}

When experiencing new symptoms, we wonder; what is wrong with me? This question relates to the diagnosis of disease, which is crucial for further treatment and prognosis (Whiting et al., 2008). The diagnosis is often reached through an investigation of patient history and diagnostic tests that can include clinical examination and imaging procedures (Whiting et al., 2008). The information will aid the clinician in making a proper diagnosis (Weinstein et al., 1980), thus benefiting the patient through an appropriate patient management process (Fryback \& Thornbury, 1991, Whiting et al., 2008) and prognosis (Whiting et al., 2008). Accurate diagnosis is also needed for professional communication, documentation, quality assurance, clinical research, theory building, and legal and reimbursement purposes (Kottner et al., 2011b).

But, clinical judgements and decisions on diagnosis are made under conditions of uncertainty. They are hampered by errors in clinical data, variations of interpretation, ambiguities in results, and uncertainties about relations between clinical information and presence of disease as well as about the effects of interventions (Weinstein et al., 1980).

The uncertainties about the relationship between clinical information and presence of disease relates to the diagnostic accuracy of tests. Diagnostic tests should ideally provide information that enables the patients to be divided into two groups - those who have and those who do not have the target condition, this will enable the clinician to decide on who will need treatment and who will not. Then, the diagnostic test can modify our prior expectation that the patient in question has the disease (Weinstein et al., 1980). Characteristics of diagnostic tests are quantified in diagnostic accuracy studies, comparing the results of the index test, the diagnostic test under 
evaluation, with the results of the reference standard, previously known as the "gold standard" (Leeflang et al., 2008; Whiting et al., 2008). For a robust reference standard, three universal criteria should be fulfilled. As formulated by Hinze \& Wenzel (2003) "it should (1) be reproducible, (2) reflect the patho-anatomical appearance of the disease to be detected, and (3) be independent of the diagnostic tests under assessment".

\section{Quantification of Probabilities and Uncertainties}

Probability is a perception of the decision-maker. The results of a test alter the decision-maker's perception of how probable it is that the patient has the disease in question. The first probability is that of a positive $(\mathrm{pT}+)$ or negative ( $\mathrm{pT}-$ ) test result. The second probability is that the patient has $(\mathrm{pD}+)$, or does not have the disease (pD-). The third type of probability is the conditional probability that the patient has the disease $(\mathrm{pT}+\mathrm{D}+)$, or does not have the disease (pT-D-) given the content of the clinical information (Weinstein et al., 1980) (Figure 2).

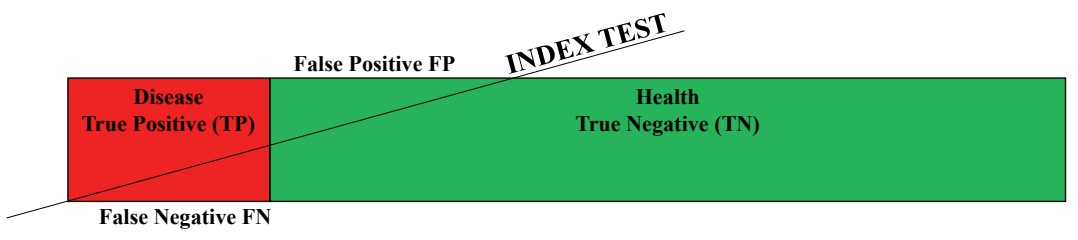

Figure 2. Visualisation of a population healthy (green) and diseased (red) individuals and the index test dividing them into four subgroups, true positive (TP), false positive (FP), false negative (FN), true negative (TN).

The true positive rate (TPR), or the sensitivity $(\mathrm{pT}+\mathrm{D}+)$, is the proportion of patients with the disease who have a positive test result (Figure 2). The true negative rate (TNR), or the specificity (pT-D-), is the proportion of patients without the disease who have a negative test result (Weinstein et al., 1980; Yerushalmy, 1947). As clinical decisions of diagnosis are made under conditions of uncertainty, not all patients will be included in the TPR or TNR. 
Tests have two features. The first is a measurable property related to a disease, for example radiolucency in tooth substance in bitewing radiographs relating to a caries lesion. The second is a positive criterion, a threshold value or cut-off point. This is a value of the measurable property distinguishing patients defined in the decision-making process as healthy/normal/acceptable from the diseased patients (Weinstein et al., 1980). The threshold could be caries visualised as radiolucency in enamel or in dentin. By defining this criterion, we dichotomise the results into only two categories; but the disease may or may not have numerous possible threshold values. This threshold, or thresholds, subsequently affects the distribution of patients in the pT-D- and $\mathrm{pT}+\mathrm{D}+$ groups.

Then, how about the uncertainties? As far as we know, no test is perfect. Therefore, some patients with the disease will exhibit negative test results (false negative rate, FNR) and some patients without the disease will exhibit positive test results (false positive rate, FPR) (Weinstein et al., 1980). These can be visualised using a cross tabulation, also called $2 \times 2$ table, from where measures of test accuracy can be derived (Figure 3).

Beside commonly known measures of sensitivity and specificity, it is possible to calculate the predictive values and likelihood ratios from such tables (Loong, 2003; Mulherin \& Miller, 2002). The probability of an index test giving the correct diagnosis for a patient is not provided by the sensitivity and specificity in the clinical situation (Altman, 1984). Instead, data from the test result itself when performed for a patient needs to be considered. The positive predictive value (PPV) is defined as the proportion of patients with a positive test result who are correctly diagnosed, and the negative predictive value (NPV) as the proportion of patients with a negative test result that are correctly diagnosed (Altman, 1984). This gives information on the test's usefulness in practice. The PPV and the NPV are commonly known to be adversely affected by disease prevalence (Altman, 1984) and this is also true for sensitivity and specificity (Bossyut et al., 2015; Feinstein 2002; Lachs et al., 1992; Leeflang et al., 2013; Mulherin \& Miller 2002). 


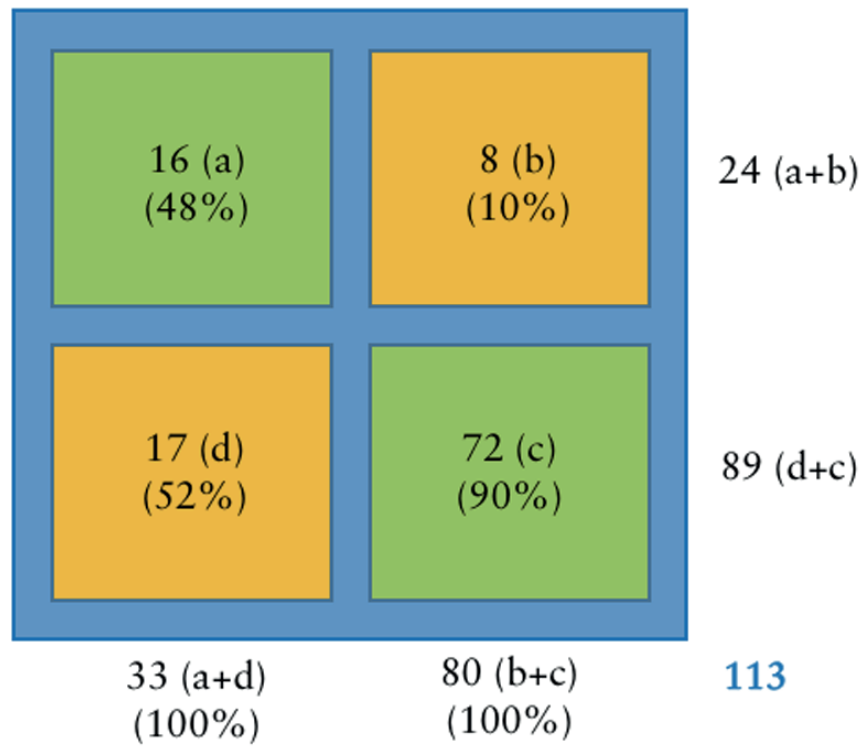

Sensitivity: $\mathrm{a} /(\mathrm{a}+\mathrm{d})=16 / 33=0.48$

Specificity: $c /(b+c)=72 / 80=0.90$

Predictive value positive: $\mathrm{a} /(\mathrm{a}+\mathrm{b})=16 / 24=$ 0.67

Predictive value negative: $\mathrm{c} /(\mathrm{d}+\mathrm{c})=72 / 89=$ 0.81

Likelihood ratio positive (LR+):

Sensitivity $/(1-$ Specificity $)=0.48 / 0.10=4.80$

Likelihood ratio negative (LR-):

$(1-$ Sensitivity $) /$ Specificity $=0.52 / 0.90=0.58$

Figure $3.2 \times 2$ table of true positive (a), false positive (b), false negative (c), true negative (d) individuals (numbers from study IV, threshold DFS $\geq 3$ for index test previous caries experience and reference standard DS $\geq 1$. Estimates for calculating test accuracy (sensitivity, specificity, predictive values and likelihood ratio). 
The likelihood ratio (LR) (positive likelihood ratio $((\mathrm{pT}+\mathrm{D}+) /$ $(\mathrm{pT}+\mathrm{D}-))$ and negative likelihood ratio $((\mathrm{pT}-\mathrm{D}+) /(\mathrm{pT}-\mathrm{D}-))$ is defined by Grimes and Schulz (2005) as "the percentage of ill people with a given test result divided by the percentage of well individuals with the same result" (Figure 3). Preferably, ill people should have a high percentage of positive test results and the positive likelihood ratio should be high. The size of the discrepancy is therefore of clinical importance (Grimes \& Schulz, 2005). Likelihood ratios have the advantage of incorporating all four cells of a $2 \times 2$ table and, according to Grimes and Schulz (2005), likelihood ratios refine clinical judgment stating that "application of a likelihood ratio to a working diagnosis generally changes the diagnostic probability, sometimes radically". Other common measures of diagnostic accuracy are the diagnostic odds ratios (ORs) and summary receiver operating characteristic curves (ROC-curves). In the ROC-curve, the FPR (1-specificity) is plotted against TPR (sensitivity) and the area under the curve indicates the test accuracy. Sub-group variation of sensitivity and specificity can also be examined by choosing a group based on relevant study characteristics and employing a chi-square test of association (Mulherin \& Miller, 2002).

\section{Validity, Reliability and Agreement}

Validity refers to the ability of a test or method to actually measure what is intended referring to the true state of the attribute estimated by a suitable reference standard (Chien \& Khan, 2001). A high validity requires a high reliability, but the reliability of a method can be high even though validity is low (SBU, 2004).

The last century's technical progress challenges the human eye and brain. Errors and variations in interpretation create a weak link and it directly affects the value of diagnostic tests and other measurements in clinical practice (Robinson, 1997). Errors or variations in interpretation have to be taken into account when evaluating methods and in any diagnostic yield. Numerous studies of reliability and agreement show a lack of transparency and incomplete reporting on crucial items (Kottner et al., 2011b). Because of inadequate reporting, interpretation and synthesis of the results becomes difficult (Kottner et al., 2009; Kottner \& Dassen 2008; Ratanawongsa et al., 2008; Stochkendahl et al., 2006). 
It has been argued that there are important differences between agreement and reliability (Streiner \& Norman, 2008; de Vet et al., 2006). Kottner and Streiner (2011) suggest that much of the confusion surrounding reliability and agreement estimation was and is caused by conceptual ambiguities. Reliability is defined as the ability of scores of a measuring device to differentiate among subjects or objects (Kottner et al., 2011a). It answers the question "How reliable is the measurement?" In other words, how well can patients be distinguished from each other, despite measurement errors? de Vet et al. (2006) defines reliability as follows: "reliability parameters assess whether study objects, often persons, can be distinguished from each other, despite measurement errors".

Agreement is defined as the degree to which scores or ratings are identical, i.e. how close the results of the repeated measurements are, by estimating the measurement error in repeated measurements (Kottner et al., 2011a). This is useful for clinicians because it answers the question "How good is the agreement between repeated measurements?" This concerns the measurement error and assesses exactly how close the scores for repeated measurements are (de Vet et al., 2006). Thus, it reflects the degree to which scores or ratings are identical between raters (inter-rater agreement) or between different points in time for the same objects and rater (intra-rater agreement).

There are several statistical approaches used in literature concerning the measurement of reliability and agreement according to GRRAS (Kottner et al., 2011a). Kappa statistics, intra-class correlation coefficients (ICC) and weighted kappa are examples of commonly statistical approaches. Agreement is also measured as percentage agreement (Kottner et al., 2011a; de Vet et al., 2013).

\section{Caries Lesion Detection}

Caries lesions can be detected by visual and tactile examination and/ or by using radiography. Gimenez et al. (2015) found that visual inspection presents good accuracy in the detection of carious lesions in teeth, with a trend towards a higher specificity than sensitivity. However, limited external validity and heterogeneity in results were present (Gimenez et al., 2015).

Bitewing radiography is "still state-of-the-art as an adjunct method in diagnosing carious lesions in clinically inaccessible approximal 
surfaces" (Wenzel, 2014). Its advantages include the possibility to detect approximal enamel lesions and the images provide a permanent record that permits assessment of longitudinal monitoring of caries lesions (Pitts, 1991). Visual and tactile examination in combination with radiography shows the highest accuracy for detection of caries lesions (SBU, 2007). In a study by Hintze and Wenzel (1993) of surfaces that first underwent clinical examination and then bitewing radiography, radiography detected enamel caries in $9 \%$ of the clinically sound approximal surfaces, and dentinal caries in $1 \%$. No histological validation was performed. This is in line with Pitts (1991) who found numerous additional approximal lesions including all severities when adding bitewings to the clinical examination; numbers were fewer of including dentinal lesions. Pitts (1991) concluded that "for posterior approximal surfaces, radiographic diagnosis will inevitably detect many more lesions than clinical examination alone".

According to a recent meta-analysis (Schwendicke et al., 2015), the sensitivity for radiographic caries detection of approximal lesions (any severity) in clinical studies was 0.24 to 0.42 and corresponding specificity was 0.70 to 0.97 . For approximal dentin lesions, sensitivity was higher, 0.36 and specificity was 0.94 .

There are different methods for validation of caries lesions, such as clinical examination, radiography, and histology (Hinze \& Wenzel 2003; Wenzel et al., 1993). For an in vitro evaluation of the accuracy of caries diagnostic methods, sectioning of the teeth with an assessment of the sections in a stereomicroscope has been confirmed to be an accurate validation (performed for occlusal caries lesions) (Hintze et al., 1995).

It is widely accepted that limited agreement is inherent to radiographic caries diagnosis (Wenzel \& Hintze, 1999a). Reported rater agreement varies among radiographic studies of caries detection (Pliskin et al., 1984; SBU, 2007; Senel et al., 2010; Wenzel, 2001). This may be owing to differences in the underlying sample characteristics, such as lesion depth, dentition, surface location, and caries prevalence, and to methodological heterogeneity, such as the number of surfaces, number of raters, and scoring categories.

There is ongoing research on the development of new clinical methods for caries detection, with varying accuracy. For this thesis, other more uncommon methods for caries detection occasionally 
included in caries prediction models, for example, fiber-optic transillumination or infrared trans-illumination technology, will not be considered.

\section{Caries Lesion Classification}

Caries lesion classification may facilitate patient-care giver communication and guide dental practitioners in treatment planning and prognosis assessments. Also, it is important for policy makers to have a reliable picture of the caries situation and development in a specific population. Giminez et al. (2015) found that studies using well-established scoring systems showed higher accuracy in detecting carious lesions in the proximal and occlusal surfaces of teeth when compared with studies that did not report the method used or that used their own criteria.

Since the late nineteenth century numerous systems for caries lesion classification have been developed. Ismail et al. (2004) investigated the content validity of a sample of caries detection criteria reported in the literature between January 1, 1966, and May 1, 2000. They found 29 criteria systems with considerable variability in disease processes measured, inclusion and exclusion criteria, and examination conditions. The most widely used carious lesion classification system is the one proposed by the WHO (WHO $4^{\text {th }}$ ed.). It includes clinical examination and caries is registered when cavitation is present. Its purpose is to allow meaningful comparison of the caries situation in various populations. When the diagnostic threshold was changed to include all lesions, DMFT values almost doubled in an examination of Hong Kong children as it went from 3.0 with dentinal lesions to 5.9 including enamel lesions (Pitts \& Fyffe, 1988) and in Scottish schoolchildren a similar discrepancy (Rimmer \& Pitts, 1991) was found when using clinical examination.

In 2001 a group of dental researchers took the initiative to develop a caries assessment system called ICDAS, based on visuaV tactile examination (Ismail et al., 2007). ICDAS is the result of an attempt to find a common caries assessment system based on the many available systems. This system includes codes for recording stages of carious lesions in enamel and in dentine, each in ascending order of severity.

For radiographic studies a classification system of approximal decayed and filled surfaces was presented by Gröndahl et al. (1977). 
Enamel caries lesions were included in this system, which was demonstrated to offer advantages compared with systems only taking new lesion into account.

\section{Caries Lesion Progression}

In general dental care, clinicians often have access to radiographs of their patients from different years. Such radiographs facilitate the assessment of changes of hard tissues over time. Clinical decisions on interventions can then be based on not only caries lesion detection but also on the assessment of caries lesion progression

Assessment of caries lesion progression is crucial also in studies of caries risk assessment, as caries increment, i.e. the development of new or more severe disease, comprises the reference standard of choice. For caries risk assessment studies, the caries increment is established through subtraction of baseline caries experience (number of lesions) from the results at the follow-up, in this way the number of new lesions is calculated (Mejàre et al., 2014). It is common to assess the reference standard through a visual and tactile examination (Bankel et al., 2011; Demers et al., 1992; Grindefjord et al., 1995b). The accuracy of this method has been deemed inadequate, for approximal lesions and for assessment of caries progression (SBU, 2007).

In view of the clinical and scientific importance of the radiographic assessment of caries lesion progression, an established classification system for this assessment could prove valuable. Accuracy of radiographic assessment of caries lesion progression in terms of sensitivity and specificity is difficult to achieve owing to difficulties in obtaining a reference standard. Wenzel et al. (1998) stated, "when no valid expression of the true state of the disease can be obtained a study of precision... is appropriate". Swets and Pickett (1982) also highlighted that observer performance could be considered as a measure of the extent to which the inaccuracy of a system is the result of decision-making errors. The level of agreement among raters for radiographic assessment of caries lesion progression is however, largely unknown. Only one previous study was found regarding the agreement of caries lesions progression (Wenzel et al., 2000). Based on the reported variation among dentists in the detection and depth estimation of caries lesions, it can be expected that this variation may also exist for the assessment of caries lesion progression. 


\section{Caries Risk Assessment Methods}

Contemporary caries risk assessment methods include previous caries experience, salivary parameters such as buffering capacity and salivary flow rate, oral hygiene measures such as plaque amount, tests using microbiota such as amount of genotypes in saliva or from dental biofilm, most common amount of S. mutans and Lactobacillus, dietary measures such as frequency of intake and/or economic- or socio-demographic variables such as educational years (Mejàre et al., 2014: SBU, 2007; Tellez et al., 2013a; Twetman, 2016). A perfect risk marker has a sensitivity of $100 \%$ and a specificity of $100 \%$, implying no errors in risk assessment. Unfortunately, no such caries risk assessment method exists. In general, the validity of caries risk assessment methods remains uncertain (Mejàre et al., 2015; Tellez et al., 2013a).

Previous caries experience is probably the most used and evaluated method to identify individuals with increased risk of dental caries lesions. This may be due to it being readily accessible, as it constitutes an integral part of the clinical examination. The method includes a visual- or a visual and tactile examination of teeth and, more uncommon in the scientific literature, bitewing radiography (Mejàre et al., 2014). Previous caries experience is considered to be the single best predictor in children (Mejàre et al., 2015; Tellez et al., 2013a; Hausen 1997; Zero et al., 2001). Baseline caries prevalence as index test performed better in pre-school children than in schoolchildren (Mejàre et al., 2015). For pre-school children, the sensitivity was $80 \%$ and specificity $79 \%$ to predict future caries lesions. In school children, specificity was higher than sensitivity (SBU, 2007). For children, the quality of the evidence was generally low and the studies were methodologically deficient (SBU, 2007). Tellez et al. (2013a) found only one risk assessment method that was validated, the Cariogram (multifactorial model), and it was found to be clinically useful in identifying caries risk levels using cavitation as disease threshold (Campus et al., 2012; Tellez et al., 2013a). Still, the external validity for this method, inside and outside Scandinavia, is unknown (Tellez et al., 2013a), this is probably the case for external validity in most caries risk assessment methods. 
Salivary secretion rate and buffer capacity were found to have a small impact on caries prediction in a multifactorial model (Cariogram) when predicting new caries lesions in children (Petersson et al., 2010). This is in line with the results of a systematic review (Mejàre et al. 2014) that there was not any increase in the predictive potential in models when implementing buffer capacity. Furthermore, there was no evidence for sociodemographic/economic methods, buffering capacity, salivary flow rate and oral hygiene measures as single factors for caries prediction. These methods showed low and scattered accuracy values (Mejàre et al., 2014).

Microbiological methods in caries risk assessment methods often includes measurements of amount of genotypes present in dental biofilm or in saliva, collected with, for example, a swab or a spatula (Mejàre et al. 2014; SBU 2007) possibly assessed with different threshold values (Kingman et al., 1988). Frequently, mutans streptococci or lactobacilli are evaluated as index tests in caries risk assessment studies (Bankel et al., 2011; Demers et al.1992; MattosGraner et al., 2000; Meurman \& Pienihäkkinen, 2010; Mejàre et al., 2014; SBU 2007). Mutans streptococci as a single predictor show inadequate accuracy for caries development in children (SBU, 2007). 


\section{OBJECTIVES}

The objectives of this thesis were to:

- Evaluate the diagnostic accuracy of different methods used to identify individuals with increased risk of developing coronal caries lesions (study I)

- Design and pilot a novel classification system for the assessment of caries lesion progression in bitewing radiography and to report rater agreement of the system (study II)

- Assess intra-individual differences in biofilm acid-tolerance between different tooth surfaces and inter-individual variation as well as stability of acid tolerance over time (study III)

- Estimate threshold effects on diagnostic accuracy of (i) previous caries experience, (ii) dentists' risk assessments and (iii) acid tolerance of dental biofilm to identify adolescents with increased risk of developing coronal caries lesions (study IV). 


\section{MATERIAL AND METHODS}

Firstly, scientific literature on methods used to identify individuals with an increased risk for caries development was critically evaluated through a systematic literature review (I). Thereby, knowledge gaps within the field were identified. The results from the systematic review influenced the planning, design, and reporting of studies II, III and IV. In an observer study (II), a novel classification system for radiographic assessment of caries lesion progression was designed and evaluated with regards to rater agreement. Study III constituted a clinical and laboratory pilot trial, evaluating a candidate method for identifying individuals with increased risk for caries development, acid tolerance of dental biofilm, with respect to distribution and stability in adolescents. Study IV comprised a prospective clinical trial on three methods to identify individuals with increased risk for caries development.

Knowledge on common deficiencies in study design and reporting from study I was used to avoid some pitfalls. The classification system for assessing caries lesion progression (study II) was implemented as one part of the reference standard (studies III and IV) and the acid tolerance of dental biofilm (study III) applied as one of three caries risk assessment methods (study IV). Study IV was designed to validate established methods on caries risk assessment, such as previous caries experience, which was identified as a necessity in future studies in the systematic review (study I).

Study II, III and IV involved adolescents from two public dental health clinics in the counties of Blekinge and Kronoberg, Sweden. The studies II, III, and IV followed the Declaration of Helsinki guidelines. Ethical approval was granted by the Regional Ethical Review Board, 
Lund, Sweden (studies II and IV, registration number: OD62-2012/122, study III registration number: 2016/146). Subjects in study III and IV received compensation upon completion of their participation.

\section{Guidelines}

In order to achieve an approach that was accurate and transparent, thus enabling the readership to assess potential bias, applicable guidelines were considered (Table 1). A modified QUADAS-2 protocol for risk of bias (low, high or unclear) was applied in four domains (study I): (i) sample selection, (ii) index test, (iii) reference standard, and (iv) flow and timing (Whiting et al., 2011). In accordance with QUADAS-2, the first three domains were also assessed for concerns regarding applicability. Protocols were piloted according to the problem specification of the review and different protocols were designed for the different index tests. Studies deemed to present a high risk of bias concerning the index test or reference standard were excluded from the final analysis in the review. The quality of evidence based on results of three or more studies was rated in four grades (high, moderate, low, very low) according to GRADE (Atkins et al., 2004). Limitations in study quality, inconsistency of results across studies, uncertainty about directness of the evidence, imprecise or sparse data and high risk of reporting bias lowered the grade of evidence. "Directness refers to the extent to which the people, interventions, and outcome measures are similar to those of interest" (Atkins et al., 2004).

\section{Systematic Review}

PubMed and two additional databases were searched. After reviewing the retrieved abstracts, selected publications were assessed in full-text according to inclusion and exclusion criteria formulated in accordance with recommendations from Centre for Reviews and Dissemination (CRD, 2009). Inclusion criteria were: primary studies/systematic reviews with a prospective and longitudinal design ( $\geq 1$ year), attrition $<40 \%$ and clinically applicable methods described to permit replication. Reference standard was caries increment/development. Data on included methods should allow for calculation of diagnostic accuracy. To facilitate comparison of results from different studies of the index test salivary mutans streptococci, the values concerning bacterial counts presented in the publications were re-calculated to counts per ml saliva. 
Table 1. Guidelines applied: PRISMA (Preferred Reporting Items for Systematic Reviews and Meta-Analysis) (Moher et al., 2010), AMSTAR (Assessing the Methodological Quality of Systematic Reviews) (Shea et al., 2007), CRD guidance for undertaking reviews in health care (Centre for Reviews and Dissemination, 2009), QUADAS-2 (Quality Assessment for Diagnostic Test Accuracy Studies) (Whiting et al., 2011), GRADE (Grading of Recommendations Assessment, Development and Evaluation) (Atkins et al., 2004), STARD (Standards for the Reporting of Diagnostic Accuracy Studies) (Bossyut et al., 2003), GRRAS (Guidelines for Reporting Reliability and Agreement Studies) (Kottner et al., $2011 \mathrm{a}$ ).

\begin{tabular}{lcccc}
\hline $\begin{array}{l}\text { Study/ } \\
\text { guideline }\end{array}$ & $\begin{array}{c}\text { Systematic } \\
\text { review }\end{array}$ & $\begin{array}{c}\text { Observer } \\
\text { study }\end{array}$ & $\begin{array}{c}\text { Clinical trial/ } \\
\text { laboratory } \\
\text { study }\end{array}$ & $\begin{array}{c}\text { Prospective } \\
\text { clinical trial }\end{array}$ \\
\hline PRISMA & $\mathrm{x}$ & & & \\
AMSTAR & $\mathrm{x}$ & $\mathrm{x}$ & & \\
CRD & $\mathrm{x}$ & & & $\mathrm{x}$ \\
QUADAS-2 & $\mathrm{x}$ & & $\mathrm{x}$ & $\mathrm{x}$ \\
GRADE & & & & $\mathrm{x}$ \\
STARD & & $\mathrm{x}$ & & \\
GRRAS & & & & \\
\hline
\end{tabular}

\section{Sample}

Adolescents, aged 12, visiting two public dental clinics, one in Kronoberg (clinic A) and one in Blekinge County (clinic S) (studies II, IV), Sweden, were approached during their regular dental check-up appointments. Individuals fulfilling the inclusion criteria were consecutively enrolled. For the purpose of an ongoing intervention study, some adolescents were randomly allocated to an intervention group. In addition to their normal oral hygiene routines, the patients drank a dose of fluoride solution $(0.75 \mathrm{mg}$ for children aged 12 years and $1.0 \mathrm{mg}$ for children $>13$ years) in $200 \mathrm{ml}$ of cow's milk or water on a daily basis. Patients in another group consumed $200 \mathrm{ml}$ fluid without fluoride daily. Between baseline and the 1-year follow-up, one general dental practitioner (GDP) at each clinic assessed the need for additional interventions individually and their decision was based 
on the results of the visual-/tactile and radiographic examination and was performed in accordance with the guidelines of each public dental clinic.

\section{Clinical, Radiographic and Microbiological Examinations}

All clinical examinations of individuals were performed by one and the same GDP at each clinic at baseline and follow-up (study IV). The clinical examinations included each tooth surface being examined separately (all surfaces of incisors and cuspids; occlusal, buccal and lingual surfaces of premolars and molars). The surfaces were scored according to ICDAS (Ismail et al. 2007), with a score from 0 to 6 . At baseline, radiographic assessment was performed of the approximal surfaces of the posterior teeth in bitewing images of right and left side (all visible mesial and distal surfaces) by two oral- and maxillofacial radiologists in consensus (study IV). Surfaces were assessed at baseline as sound, caries lesion in the outer enamel, caries lesion in the inner enamel (reaching the dento-enamel junction) and caries lesion in the dentine.

In studies II and IV the equipment (dental X-ray units and imaging plate systems) was checked for the following parameters prior to the radiographic examinations: tube voltage, exposure time (reproducibility and linearity), low contrast, and spatial resolution. The doses of radiation (microgray) for different exposure times were recorded. In addition, dose ratios (milligray per second) were recorded for applicable exposure times $(0.2,0.25$ and $0.32 \mathrm{~s})$. Regarding tube voltage, thresholds for kilovoltage were adjusted $(60,63,66$ and 70 $\mathrm{kV}$ ) and the actual tube voltage was recorded with RaySafe ThinX Intra equipment (Unfors RaySafe, Fluke Biomedical, WA). When exposure times were registered, a scheme of five repetitions was adopted for the reproducibility control.

Further, a series ranging from 0.01 to $0.5 \mathrm{~s}$ of exposure time was used for the linearity control. Doses of radiation were measured throughout and dose ratios calculated on the obtained values. Spatial resolution was measured by exposing a spatial resolution phantom three times with different exposure times intended to produce optimal visualization of the object in the computer software used. Two plexiglass plates served as a phantom meant to represent the soft tissues of patients, thus being placed in between the spatial resolution 
phantom and the imaging plate. A value, visible lines per millimetre, was obtained by reviewing the radiographic image (expressed as a number of 1-10). In order to obtain the best possible image of low contrast reproducibility, a plexiglass rectangle with depressions of different depths was used. All images were exported as digital imaging and communication in medicine files (DICOM) to portable storage media from the digital viewing software programs. The images were then imported to Romexis ${ }^{\circledR}$ software (Planmeca, Finland) and were anonymised (studies II, IV).

For the microbiological examinations, all collections of dental biofilm were performed by one and the same GDP at clinic A and $S$ (study IV) respectively at the time of baseline examinations. The dental biofilms were sampled with Quicksticks (Dab Dental AB, Upplands Väsby, Sweden). Each sample was transferred into a separate sterile microfuge tube, sealed, and sent to the laboratory for further analysis. Laboratory method included each sample from the clinic being exposed to acid shock at $\mathrm{pH} 3.5$ and after incubation the cells were stained using LIVE/DEAD ${ }^{\circledR}$ BacLight $^{\mathrm{TM}}$ Fluorescent Stain (Molecular Probes, Eugene, USA). Each biofilm sample was examined using an inverted confocal scanning laser microscope (Nikon Eclipse TE2000, Nikon Corp., Tokyo, Japan). Live bacteria (acid tolerant) appear green, while dead (non-acid tolerant) bacteria appear red. For every adolescent, ten random images were recorded from each of the pooled dental sites on each occasion. All ten images were scored separately by one oral microbiologist according to an interval scale (Figure 4) with 5 possible scores representing the proportion of acid tolerant cells in the biofilm (studies III, IV). The most representative score from the ten images was used to represent the sample (III, IV).

For half of the individuals in study III $(\mathrm{n}=20)$ samples were taken from all supragingival approximal surfaces between second premolars and first molars (four sites) and pooled to give one sample for each individual. Samples were taken at baseline and after 6 and 12 months. In the other half of the individuals $(n=20)$ dental biofilms were sampled and pooled in the same manner from all approximal surfaces between second premolars and first molars (four sites), between canines and first premolars (four sites) and between the central incisors in both jaws (two sites). This sampling took place at baseline, after three days and after one month. In study III, a change 


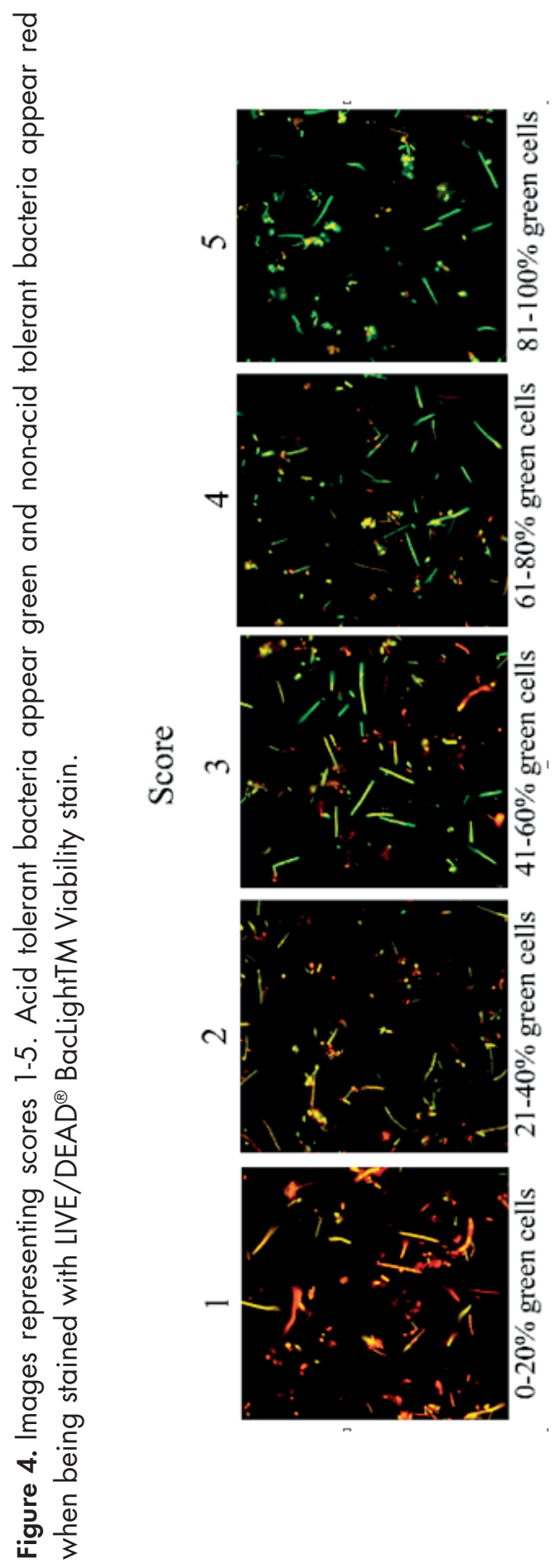


in the level of acid tolerance was defined as an increase or decrease of $\geq 2$ scores according to the interval scale. This corresponded to a difference of at least $20 \%$ in the proportion of acid-tolerant cells in the images.

\section{Evaluation of Diagnostic Methods}

Diagnostic methods were evaluated with regards to diagnostic accuracy and rater agreement. Diagnostic accuracy of methods for caries risk assessment was calculated through cross-classifying the number of true positive (TP), false positive (FP), false negative (FN), and true negative (TN) individuals for available (I) or selected threshold values (IV). Threshold values for the index tests and reference standard were pre-specified in study IV based on: thresholds assumed relevant for clinical practice, current risk assessment program of the public dental service in one region (Blekinge), and on acid tolerance scores implemented in an ongoing intervention study.

The sensitivity, specificity, predictive values and likelihood ratios, along with their corresponding $95 \%$ confidence intervals were calculated from the cross-classifications. In study I, forest plots on accuracy measures were generated for selected subgroups of individuals using the statistical software Meta-DiSc (Zamora et al., 2006). Heterogeneity was assessed visually, using the forest plots, and statistically, using a chi-square test, with $\mathrm{P}<0.05$ indicative of heterogeneity across studies.

Available measures of rater agreement were extracted (study I) or assessed for the classification system on caries lesion progression (study II) and for caries detection (study IV) as well as for acid tolerance (studies III, IV). The assessments included kappa values, with $95 \%$ confidence intervals, for intra-rater (studies II, III, IV), inter-rater (studies II, IV) and pair-wise rater agreement (studies II, IV) as well as agreement in percentage for intra-rater and pair-wise rater agreement. Inter-rater agreement calculations were based on the first reading (studies II, IV). Kappa values, varying from -1 to 1 , were categorised according to the scale suggested by Landis and Koch (1977). According to this scale, values less than zero are considered as poor agreement, 0.00 to 0.20 as slight, 0.21 to 0.40 as fair, 0.41 to 0.60 as moderate, 0.61 to 0.80 as substantial, and values higher than 0.81 as almost perfect agreement. 


\section{Previous Caries Experience}

Previous caries experience in study IV was based on the visual and tactile examination of all permanent teeth and bitewing radiography of the posterior teeth. The pre-specified thresholds of previous caries experience were defined as:

- DMFS $\geq 1, \geq 2, \geq 3, \geq 4$ or $\geq 5$

Decayed Missing Filled Surfaces of permanent teeth. Decayed included all coronal surfaces and caries lesion severities, missing were included if it was due to caries. Only fillings due to caries were included.

\section{Dentists' Risk Assessments}

Dentists' risk assessments in study IV was based on the visual and tactile examination of primary and permanent teeth and bitewing radiography of the posterior teeth. Each tooth surface was assessed as healthy, with enamel or dentine lesion. The GDP in each clinic performed a risk assessment on the basis of the results from their previous and present clinical and radiographic examinations. Since all adolescents had attended the clinics for two years or longer, it was possible to acquire their records and assess coronal caries lesion progression during two years. The pre-specified thresholds for dentists' risk assessments were applied according to criteria implemented in one of the public dental clinics and defined as:

- low risk $=$ no new enamel caries lesion during the previous two years

- high risk $=\geq 1$ dentinal caries lesion (arrested or progressed lesion) or $\geq 1$ new approximal caries lesion in enamel during the previous two years.

\section{Acid Tolerance of Dental Biofilm}

For the purpose of using acid tolerance method as an index test for caries risk assessment (study IV) dental biofilm from all supragingival approximal surfaces between second premolars and first molars (four sites) were pooled to give one sample for each individual at baseline. The individual received one score according to the interval scale (Figure 4). 
The pre-specified thresholds for acid tolerance of dental biofilm as an index test were:

- Score $\geq 1, \geq 2, \geq 3, \geq 4$ or 5

\section{Reference Standard - Caries Lesion Progression}

To determine the reference standard in study IV, clinical and radiographic examinations were repeated after 1 year with access to records from baseline. The records of the clinical examinations at baseline and after 1 year were compared for all surfaces of incisors and cuspids and for occlusal, buccal, and lingual surfaces of premolars and molars regarding the ICDAS scores. An increase in ICDAS scores over the year was interpreted as caries lesion progression for these surfaces.

Approximal surfaces of premolars and molars were assessed according to a classification system designed to visualise caries lesion progression in terms of no progression or progression of caries lesions developed in study II. The bitewing images were displayed side-by side on a monitor in bitewing images: one image pair from baseline and one from the 1-year follow-up (Figure 5).
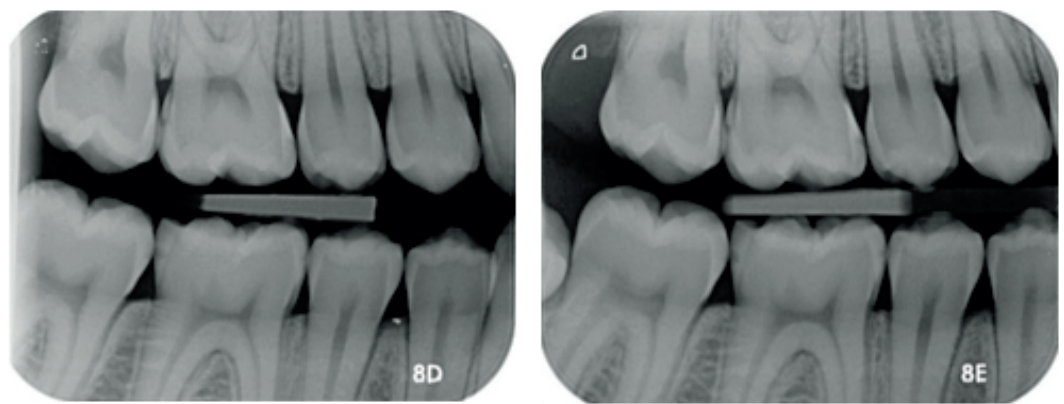

Figure 5. Examples of a bitewing image pair from study II, baseline examination left and follow-up right. 
The assessments were performed without access to the results of the clinical examinations. Figure 6 displays the system used for assessment of caries lesion progression of each approximal surface, based on the classification system designed to visualise sound, arrested and progressed caries lesions. The pre-specified thresholds for caries increment were defined as:

- DMS $\geq 1, \geq 2$ or $\geq 3$

Decayed Missing Filled Surfaces, of permanent teeth. Decayed included progressed coronal caries lesions, all coronal surfaces and caries lesion severities, missing were included if they were due to caries.

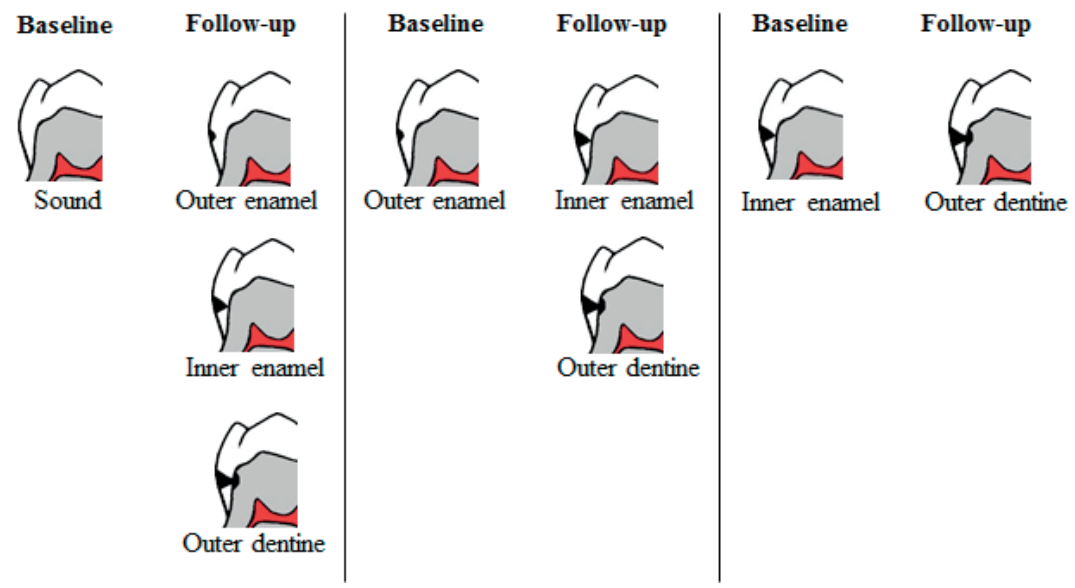

Figure 6. Modified classification system for assessment of caries lesion progression of approximal surfaces in bitewing radiography (full version presented in study III.

\section{Rater Agreement}

Radiological Assessment of Approximal Caries Lesions and Caries Lesion Progression

For study II, rater agreement of caries lesion progression, images of each selected patient were paired: one image from baseline and one image from the 1-year follow-up, resulting in 65 image pairs. The radiographs were selected to represent five surfaces for all categories 
presented in Figure 6, except for surfaces with caries lesion in the inner half of the dentine, which were not represented in the sample and not assessed by the raters. 20 sound surfaces without progression were included. Altogether, 65 approximal surfaces in the 65 image pairs with and without caries lesion progression were included for assessment. 10 raters assessed the surfaces and seven raters reassessed all surfaces in the same order and same setting after 2-4 weeks. During the second viewing, raters were blinded towards their results from previous assessments.

For study IV, intra-rater agreement of caries lesion detection and caries lesion progression, radiographs were re-assessed in the same setting after a minimum of 2 weeks. During the second viewing, raters were blinded towards the results of their previous assessments. For the assessments of caries detection, radiographs of 10 adolescents with different status concerning caries lesions $(50 \%$ adolescents with caries lesions) were selected. Surfaces were assessed as sound, caries lesion in the outer enamel, in the inner enamel (reaching the dento-enamel junction) or in the dentine. For assessment of caries lesion progression, pairs of radiographs of 10 other adolescents with different status concerning caries lesion progression $(50 \%$ adolescents with caries lesions) were selected. Caries lesion progression was assessed using a modified classification system (Figure 6) based on a classification system presented in study II.

\section{Assessment of Proportion Acid Tolerant Cells}

To establish inter- and intra-rater agreement of the assessments of proportion acid tolerant cells in images of dental biofilm, seven raters, all experienced within the field, were asked to assess 50 images of samples originating from clinic A in study III. The images were selected to represent 10 images of each score (score 1-5) (Figure 4). For inter-rater agreement, the images were presented to the raters in random order one by one. The raters independently assessed and scored each image. No calibration exercise preceded the sessions. For intra-rater agreement, two of the raters repeated their assessments after 14-21 days under the same conditions. The order of image presentation differed from the first session. Raters were blinded towards previous results. 


\section{RESULTS}

\section{Systematic Review}

From the search in three databases, 5776 publications were identified. After reviewing the abstracts, 315 were assessed in full-text and 18 publications (13 from PubMed) were included for final analysis. Many of the excluded studies focused on the correlations between caries-related factors and the development of caries. Another main reason for exclusion was the lack of data on diagnostic accuracy or on data to populate $2 \times 2$ tables.

The following methods (index tests) of identifying individuals with an increased risk of caries were investigated in the included studies: (i) previous caries experience, (ii) mutans streptococci sampled from saliva or plaque, (iii) lactobacilli sampled from saliva, (iv) buffer capacity $(v)$ salivary flow rate, (vi), dental plaque/oral hygiene, (vii) dietary habits, and (viii) sociodemographic variables. None of the included publications fulfilling the inclusion criteria clarified the risk concept. Sample size ranged from 27 to 832 individuals. Caries prevalence at baseline - based on cavity in some studies and on enamel and dentinal caries lesions in other - ranged from 0.2 to $83 \%$.

Caries lesions were mainly diagnosed using visual/visual-tactile examination at baseline, only five studies added radiography in all or selected study objects and tooth surfaces (Coogan et al., 2008; David et al., 2006; Skeie et al., 2006; Petti and Hausen, 2000; Sullivan \& Schröder, 1989). The reference standard, caries increment or caries development between baseline and follow-up ( $\geq 1$ year) was based on visual/visual-tactile examination but two studies added radiography for all study objects (Coogan et al., 2008; David et al., 2006). Regarding the other studies, implementing radiography, it 
was unclear exactly how and for which patients it was used (Skeie et al., 2006; Petti and Hausen 2000; Sullivan and Schröder 1989). Caries development included enamel and dentinal caries lesions in three studies (Coogan et al., 2008; Skeie et al., 2006; Sullivan and Schröder, 1989) and only dentinal lesions in the other two studies (Petti and Hausen, 2000; David et al., 2006).

\section{Assessment of Risk of Bias}

Assessment of risk of bias of included publications in study I using a protocol modified after QUADAS-2 (Whiting et al., 2011) identified several methodological limitations. No study presented low risk of bias in all four domains of the protocol. Most studies insufficiently reported a definition of the criteria for thresholds of the index tests and reference standard. For reference standard, poor reporting of how caries lesions and caries lesions progression was examined and assessed, resulting in "unclear risk of bias" for most studies. About half of the studies were deemed to present an unclear risk for flow and timing as withdrawals were not analysed. Five of the included studies in study I implemented radiography. The results from the assessment of risk of bias for those studies is presented in figure 7 .

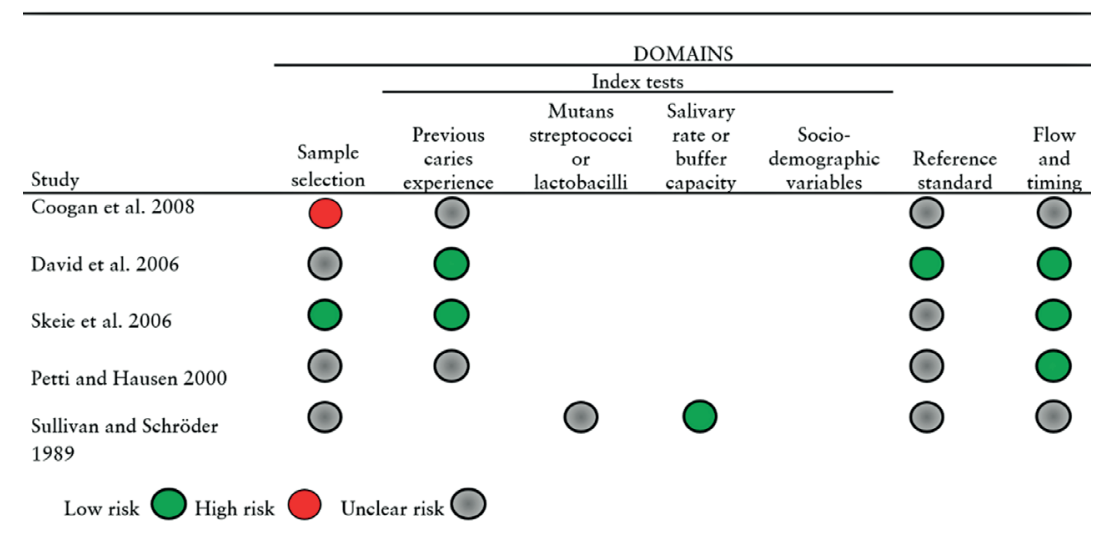

Figure 7. Risk of bias according to QUADAS-2 in four domains of the studies which included bitewing radiography for the assessment of caries lesions and caries lesion progression. 
All studies of microbiota presented unclear risk of bias for the index test since the sampling method, examination method, classification system, and method for interpreting results were unclear. Only two studies (Petti \& Hausen, 2000; Kingman et al., 1988) presented the amount of saliva sampled. Information to ascertain possible detection levels of microbiota for the method implemented was insufficient across all studies. It is unclear whether mutans streptococci were identified by their characteristic CFU-morphology as no study reported criteria for this. In only two studies (Petti \& Hausen, 2000; Kingman et al., 1988) the growth of mutans streptococci was confirmed by biochemical testing.

\section{Sample Characteristics Concerning Caries Status}

Figure 8 presents recruited, withdrawn, and included adolescents in study IV. At baseline, caries prevalence of included adolescents (all surfaces and severities) was 39\% (DFS 1.7) and 63\% (DFS 2.8) for withdrawals. Regarding dentinal lesions, prevalence was $8 \%$ for included adolescents and $23 \%$ for withdrawals. DFS of withdrawals was higher than that of included adolescents in both clinics. There were no teeth missing due to caries.

At baseline and follow-up, a majority of decayed approximal lesions were enamel lesions (for included adolescents, 86 and $78 \%$ respectively in each clinic). Caries lesion progression occurred in 25 $(33 \%)$ adolescents in clinic A and $8(21 \%)$ in clinic S, mainly in approximal surfaces and as enamel lesions. At the 1-year follow-up, caries prevalence expressed as percentage of adolescents with caries lesions was the same in both clinics $(45 \%)$ but expressed as DFS (enamel and dentinal lesions) it was higher in clinic A (2.5) than in clinic S (1.6).

\section{Acid Tolerance in Dental Biofilm}

To investigate whether acid tolerance was stable between different teeth surfaces of the same individual, biofilms were sampled on a single occasion from three different approximal sites in 20 individuals. In fourteen individuals, acid tolerance scores did not differ within the oral cavity. However, in six individuals, the acid tolerance at the premolar site differed from one of the other two sites. 


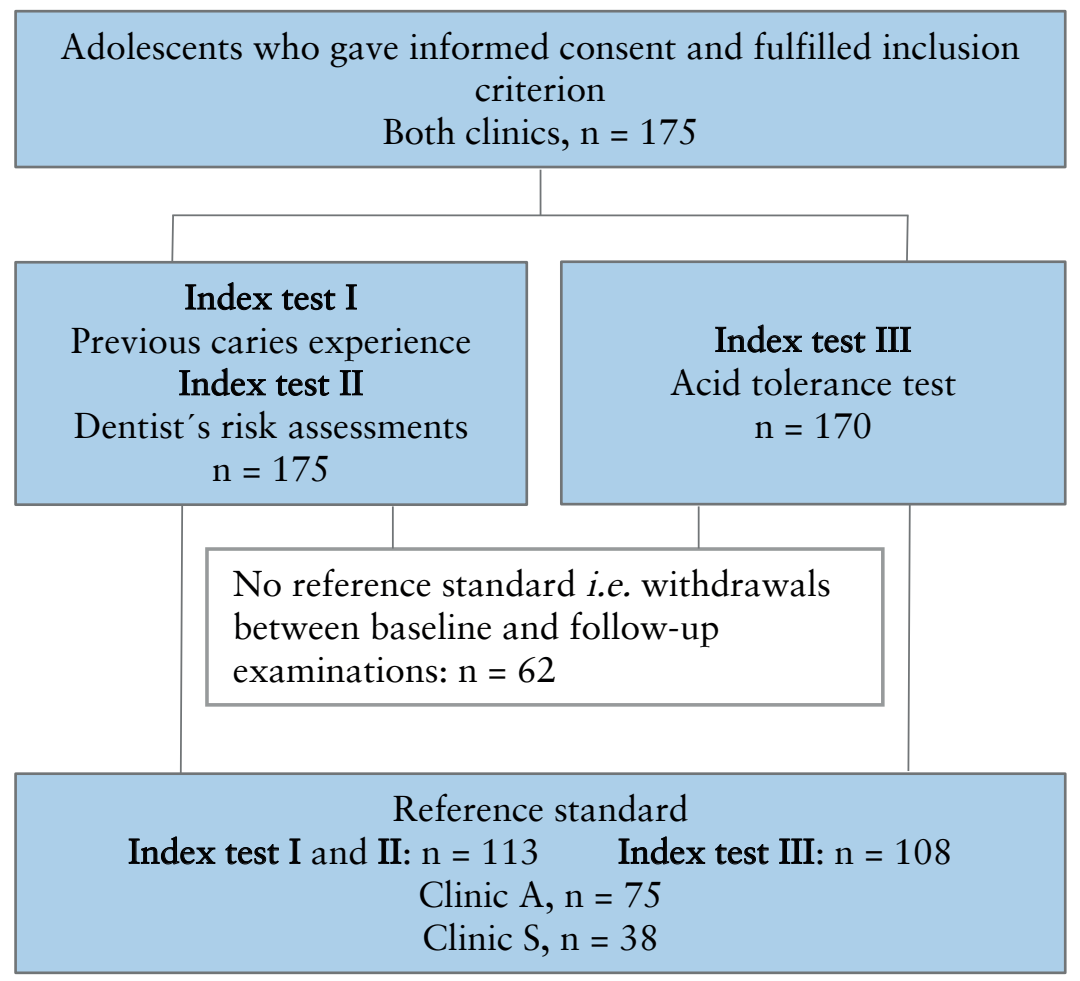

Figure 8. Modified STARD flow-chart (Bossuyt et al., 2015) of number of adolescents recruited, withdrawals and included for final analysis in study IV.

To investigate whether acid tolerance was stable over time, the values at baseline at 60 different sites ( 3 sites of 20 individuals) were compared with those obtained after 1 month. In $85 \%$ of the surfaces, low (score 1 and 2) or moderate (score 3 ) acid tolerance values at baseline and after one month were the same.

Since we postulated that caries development would require a high degree of acid tolerance in the biofilm over a considerable period, fluctuations in biofilm acid tolerance at one specific site over the longer term was also studied. $85 \%$ of the sites showed a low or moderate acid tolerance scores at baseline and the values remained low over a 1-year period. These results show that for most individuals in this study, acid tolerance was generally low and stable over the long term. 


\section{Validity of the Interval Scale and Consistency of Acid Tolerance Assessment Method}

For eight of ten images, the score assigned using the scale was exactly the same as that revealed by counting the visible bacteria. For two images, however, the rater's assessment differed by one score from that obtained by counting. In both images, the actual number of acid-tolerant cells was close to the threshold boundary, for example, an image given score 3 by the rater (corresponding to $41-60 \%$ acidtolerant cells) actually contained $62 \%$ acid-tolerant cells.

To investigate the consistency of the assessment method, sampling from between the central incisors, canine and first premolar, and second premolar and first molar sites was repeated after three days and the acid-tolerance values compared with the baseline measurements. This revealed a high degree of short-term consistency, with the same values obtained in $90 \%$ of the individuals at the incisor and molar sites and $85 \%$ of the individuals at the sites between the canines and pre-molars.

\section{Diagnostic Accuracy of Methods to Identify Individuals with Increased Risk of Caries Development}

Accuracy estimates retrieved from publications included in study I on previous caries experience varied both across studies and within the same sample. For children aged 2-2.5 at baseline and primary teeth at follow-up, pooled sensitivity was low $(0.30)$ and pooled specificity high (0.97), given thresholds of index test and reference standard $>0$ caries lesion (Bankel et al., 2011; Grindefjord et al., 1995; Pienihäkkinen et al., 2004). Pooled LR+ was 26.9 and LR0.71 (Table 2). There were few false-positive diagnoses but numerous false-negatives.

Predicting caries in permanent teeth based on previous caries experience in primary teeth (children aged $3.5-7)$ yielded higher pooled sensitivity (0.91) but low pooled specificity (0.29), given index test $\mathrm{dmft}>0$ and reference standard DMFT $>0$ (Li et al., 2002; van Palenstein Helderman 2006; Skeie et al., 2006; Zhang et al., 2006). Pooled LR+ was 1.33 at a LR- of 0.31. In this sample, false-positive diagnoses were numerous and false-negatives few. For permanent teeth (David et al., 2006) sensitivity varied between 0.21 and 0.62 given different thresholds of caries increment. 
Table 2. Pooled values and ranges of likelihood ratio positive $(L R+)$ and negative (LR-) in different subgroups of samples for index test previous caries experience. Reference standard (caries increment) threshold: $>0$ caries lesions (study I).

\begin{tabular}{lll}
\hline Sample & $\begin{array}{l}\text { Pooled LR+ } \\
\text { Range }\end{array}$ & $\begin{array}{l}\text { Pooled LR- } \\
\text { Range }\end{array}$ \\
\hline $\begin{array}{l}\text { Children, } 2 \text { - } 7 \text { yrs } \\
\text { Primary teeth at baseline }\end{array}$ & $\begin{array}{l}3.19 \\
1.17-63\end{array}$ & 0.43 \\
(7 studies) & & $0.14-0.73$ \\
Children, 2 - 7 yrs & 26.9 & 0.71 \\
$\begin{array}{l}\text { Primary teeth at baseline and follow-up } \\
\text { (3 studies) }\end{array}$ & $10.3-63.1$ & $0.43-0.73$ \\
$\begin{array}{l}\text { Children, 3.5 - 7 yrs } \\
\text { Primary teeth predicting caries in }\end{array}$ & 1.33 & 0.31 \\
permanent teeth (3 studies) & $1.17-1.75$ & $0.14-0.45$ \\
\hline
\end{tabular}

The highest accuracy estimates retrieved from publications included in study I on microbiota as index test was pooled LR +4 for threshold $\geq 10^{5} \mathrm{CFU} / \mathrm{ml}$ salivary mutans streptococci in children aged 2-7 at baseline, despite differences in the sampling techniques used in the included studies. The influence of different thresholds is evident in a study of permanent teeth (Kingman et al., 1988). Sensitivity ranged between 0.04 for $10^{6} \mathrm{CFU} / \mathrm{ml}$ and 0.75 for $>10^{2} \mathrm{CFU} / \mathrm{ml}$ with corresponding specificity 0.98 and 0.35 (Figure 9). Values on sensitivity fluctuated more depending on reference standard threshold as compared to specificity. The positive likelihood ratio ranged from 1.16 to 3.99 with corresponding negative likelihood ratio of 0.97 to 0.27 (Kingman et al., 1988).

For mutans streptococci sampled from plaque and for lactobacilli in children, accuracy estimates were similar to those for salivary mutans streptococci: sensitivity was low and specificity high. A combination of lactobacilli and mutans streptococci did not enhance diagnostic accuracy. Diagnostic accuracy of salivary rate, buffering capacity, and sociodemographic variables was low. For mother's or parents' educational level, sensitivity ranged between 0.35 and 0.69 and specificity between 0.57 and 0.77 . 


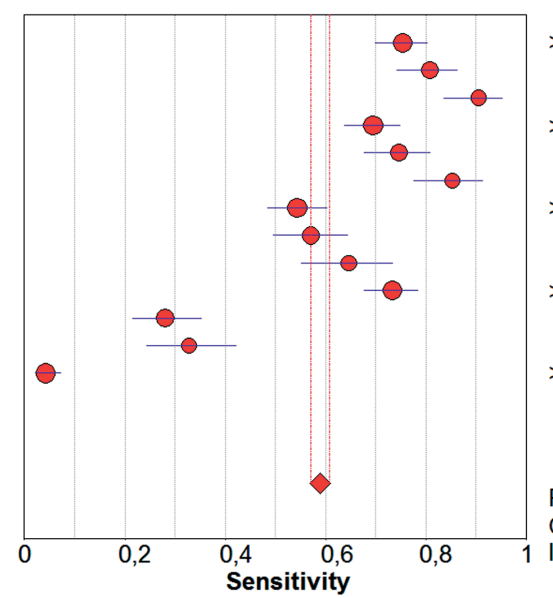

$>100 \mathrm{CFU} / \mathrm{ml}$

$>1000 \mathrm{CFU} / \mathrm{ml}$

$>10000 \mathrm{CFU} / \mathrm{ml}$

$>100000 \mathrm{CFU} / \mathrm{m}$

$>1 \mathrm{milj}$ CFU/ml

Pooled Sensitivity $=0,59(0,57$ to 0,61$)$

Chi-square $=735,98 ; \mathrm{df}=12(\mathrm{p}=0,0000)$

Inconsistency (I-square) $=98,4 \%$

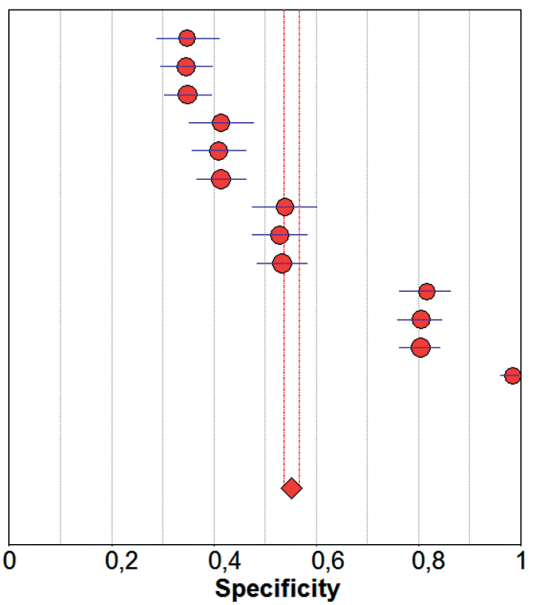

Sensitivity $(95 \% \mathrm{CI})$

$0,75 \quad(0,70-0,80)$

$0,81 \quad(0,74-0,86)$

$0,91 \quad(0,84-0,95)$

$0,69 \quad(0,64-0,75)$

$0,75 \quad(0,68-0,81)$

$0,85 \quad(0,78-0,91)$

$0,54 \quad(0,48-0,60)$

$0,57 \quad(0,50-0,64)$

$0,65 \quad(0,55-0,73)$

$0,73 \quad(0,68-0,78)$

$0,28 \quad(0,22-0,35)$

$0,33 \quad(0,24-0,42)$

$0,04 \quad(0,02-0,07)$

Pooled Specificity $=0,55(0,54$ to 0,57$)$

Chi-square $=825,37 ; \mathrm{df}=12(\mathrm{p}=0,0000)$

Inconsistency (I-square) $=98,5 \%$

Specificity $(95 \% \mathrm{CI})$

$>100 \mathrm{CFU} / \mathrm{ml}$

$0,35 \quad(0,29-0,41)$

$0,35 \quad(0,30-0,40)$

$0,35 \quad(0,30-0,40)$

$>1000 \mathrm{CFU} / \mathrm{ml}$

$0,41 \quad(0,35-0,48)$

$0,41 \quad(0,36-0,46)$

$>10000 \mathrm{CFU} / \mathrm{ml}$

$0,41 \quad(0,37-0,46)$

$0,54 \quad(0,48-0,60)$

$0,53 \quad(0,48-0,58)$

$0,53 \quad(0,49-0,58)$

$>100000 \mathrm{CFU} / \mathrm{ml}$

$0,82 \quad(0,76-0,86)$

$0,81 \quad(0,76-0,84)$

$0,80 \quad(0,76-0,84)$

$>1 \mathrm{milj}$ CFU/ml

$0,98 \quad(0,96-1,00)$

Figure 9. Forest plots presenting estimates of sensitivity and specificity and corresponding $95 \% \mathrm{Cl}$ of salivary mutans streptococci with thresholds from $>100$ to $>1000000 \mathrm{CFU} / \mathrm{ml}$. Forest plots based on data presented in the study by Kingman et al., (1988). Referencestandard (caries increment) thresholds were DMFS >0, DMFS >1, DMFS $>2$ for each index test threshold value of $\mathrm{CFU} / \mathrm{ml}$, in ascending order.

Regarding diagnostic accuracy estimates of three methods evaluated in study IV, accuracy varied between different methods, threshold values for index test and reference standard and for the samples of the clinics. The variations often followed distinctive patterns, reflecting differences in prevalence created by the threshold for disease. An 
increase in prevalence yielded an increase in sensitivity estimates and often a more moderate decrease in specificity. Follow-up prevalence expressed as percentage of adolescents with caries lesions was the same for the clinics $(45 \%)$ but accuracy estimates were not since disease spectrum differed with a higher DFS in clinic A.

Table 3. Diagnostic accuracy of selected thresholds for previous caries experience and caries prevalence (\%) correlating to each threshold (assessment included all surfaces and severities) at baseline and followup based on the sample of clinic $A$ and $S$ with corresponding accuracy estimates (study IV).

\begin{tabular}{ccccccccc}
\hline \multicolumn{2}{c}{ Threshold } & \multicolumn{2}{c}{ Prevalence (\%) } & \multicolumn{5}{c}{ Accuracy } \\
\hline $\begin{array}{c}\text { Index } \\
\text { test }\end{array}$ & $\begin{array}{c}\text { Reference } \\
\text { standard }\end{array}$ & Baseline & Follow-up & Sensitivity & Specificity & LR+ & LR- \\
\hline DFS $\geq 3$ & DS $\geq 1$ & 19 & 29 & 0.48 & 0.90 & 4.85 & 0.50 \\
DFS $\geq 3$ & DS $\geq 2$ & 19 & 12 & 0.79 & 0.87 & 5.98 & 0.25 \\
DFS $\geq 3$ & DS $\geq 3$ & 19 & 4 & 0.75 & 0.83 & 4.34 & 0.30 \\
DFS $\geq 1$ & DS $\geq 1$ & 51 & 29 & 0.76 & 0.59 & 1.84 & 0.41 \\
DFS $\geq 2$ & DS $\geq 1$ & 35 & 29 & 0.67 & 0.79 & 3.14 & 0.42 \\
DFS $\geq 3$ & DS $\geq 1$ & 19 & 29 & 0.48 & 0.90 & 4.85 & 0.50 \\
\hline
\end{tabular}

\section{Previous Caries Experience}

Diagnostic accuracy of previous caries experience as a method presented sensitivity values between 0.30 and 0.86 and specificity between 0.50 and 0.95 . Ranges for accuracy estimates were also wide for a sample within the same clinic. Negative predictive values were high (range $0.72-1$ ) being $\geq 0.90$ for many thresholds. That is, adolescents not exhibiting any previous caries experience are correctly identified with no caries development. The positive predictive values were lower in general (range 0.05 - 1). Thus, the method performs poorly at identifying adolescents with increased risk of coronal caries lesion development. For the sample in clinic $S$, high $L R+(7.5)$ and low LR- (0.3) was achieved given an index test threshold of DFS $\geq 2$ and reference standard DS $\geq 1$. With the same thresholds in clinic $A$, LR+ was 2.67 and LR- 0.47. Relatively high accuracy, in both clinics in terms of LRs, was achieved for index test thresholds DFS $\geq 4$. 


\section{Dentists'Risk Assessments}

Regarding accuracy of dentists' risk assessments, sensitivity and specificity estimates were somewhat lower than those for previous caries experience. Sensitivity for both clinics ranged between 0.33 and 0.75 and specificity between 0.81 and 0.84 . Most negative predictive values were around 0.90 whilst only two positive predictive values were $\geq 0.50$. For the whole sample, the best LRs were LR+ 3.9 and LR- 0.3, achieved for reference standard DS $\geq 3$.

\section{Acid Tolerance of Dental Biofilm}

For acid tolerance test, sensitivity was low and ranging between 0.04 and 0.75 , whilst specificity was higher ranging between 0.47 and 0.96. Negative predictive values were high in particular for the sample in clinic $\mathrm{S}$, whilst positive predictive values were low indicating that low acid tolerance scores were predictive of future health. All LR+ for the whole sample was $\leq 2.93$ and LR- $\geq 0.58$. For the sample in clinic $\mathrm{S}$, more favourable LRs were achieved than for the sample in clinic $\mathrm{A}$, in particular for score $\geq 4$ given reference standard DS $\geq 1(\mathrm{LR}+6.2$; LR- 0.17).

\section{Rater Agreement}

All included studies in the systematic review (study I), images and raters' assessments (studies II-IV) were included for analysis of reproducibility measures (Table 4). For acid tolerance (study IV) and caries lesion progression (study II), intra-rater agreement was generally higher than inter-rater agreement.

Substantial (>0.61) inter-rater agreement, as kappa values, was achieved for 10 raters for the assessment of caries lesion progression (study II). Intra-rater agreement in percentage was $69-92 \%$ depending on rater. Pair-wise agreement ranged from 0.42-0.84, with half of the values $>0.61$.

For inter-rater agreement on acid tolerance (study IV), with threshold for each score of 1-5, also resulted in substantial agreement (kappa $\geq 0.76$ ), though, threshold $1,2,3,4$ vs. 5 showed almost perfect agreement (kappa 0.89; CI 0.76 - 0.97). Pair-wise inter-rater agreement was $50-86 \%$, corresponding to fair to substantial agreement (kappa 0.38-0.83), most values being moderate to substantial agreement. 
Intra-rater agreement varied between substantial and almost perfect for all thresholds, with no major difference between raters, being $74 \%$ (kappa 0.67; CI 0.52 - 0.81) for both raters and all scores. The intra-rater agreement for all scores for the rater, who assessed samples included in study IV was $94 \%$ (kappa 0.92).

Regarding the other microbiological methods, only one included study in the systematic review (study I) presented examiner reproducibility of the index test (Demers et al., 1992). Further, for included studies in study I, the intra-examiner reproducibility of visual/visual-tactile examination varied between kappa 0.77 and 0.94 . Pair-wise interexaminer reproducibility ranged between 0.79 and 0.88 .

\section{Evidence}

Evidence was established for the methods previous caries experience and salivary mutans streptococci in study I. The quality of evidence was assessed as low according to GRADE (Atkins et al., 2004) due to heterogeneity in the results and limitations in study quality (Table $5)$. Since there were too few studies regarding other tests, evidence was lacking regarding mutans streptococci from plaque, lactobacilli, salivary parameters, and sociodemographic variables. The index tests dental plaque/oral hygiene and dietary habits were excluded from analysis based on insufficient description of index tests. 
Table 4. Kappa values with confidence intervals $(\mathrm{Cl})$ on inter- and intrarater agreement as well as agreement in percentage of caries lesion detection, caries lesion progression and acid tolerance test. Included methods are assessed in the systematic review (study I) or implemented as index tests or reference standard (studies II, III) in the study on caries risk assessment methods (study IV).

\begin{tabular}{|c|c|c|c|c|}
\hline \multirow[b]{2}{*}{$\begin{array}{l}\text { Reliability } \\
\text { measures }\end{array}$} & \multicolumn{2}{|c|}{$\begin{array}{l}\text { Caries detection, including } \\
\text { radiography } \\
\text { (studies I, IV) }\end{array}$} & \multirow{2}{*}{$\begin{array}{c}\text { Caries lesion } \\
\text { progression/no } \\
\text { progression (study II) } \\
\text { Enamel+dentine } \\
\text { lesions }\end{array}$} & \multirow{2}{*}{$\begin{array}{c}\begin{array}{c}\text { Acid tolerance } \\
\text { (study III) }\end{array} \\
\text { Score 1-5 }\end{array}$} \\
\hline & $\begin{array}{c}\text { Enamel+dentine } \\
\text { lesions }\end{array}$ & Dentine lesions & & \\
\hline \multicolumn{5}{|l|}{ Inter-rater } \\
\hline Kappa (Cl) & $\begin{array}{l}5 \text { raters: } \\
-0.71-0.88 \\
5 \text { raters: }{ }^{*} \\
-0.71-0.90\end{array}$ & $\begin{array}{l}3 \text { raters: }{ }^{* *} \\
-0.84-0.89\end{array}$ & $\begin{array}{l}10 \text { raters: } \\
\text { - } 0.61 \\
\text { (range } 0.54-0.70 \text { ) } \\
\text { Threshold: } \\
\text { - sound } 0.78 \\
\text { - enamel } 0.44 \\
\text { - dentin } 0.55\end{array}$ & $\begin{array}{l}7 \text { raters: } \\
0.59 \\
(0.50-0.69)\end{array}$ \\
\hline \multicolumn{5}{|l|}{ Intra-rater } \\
\hline Kappa (Cl) & $\begin{array}{l}\text { Baseline, } \\
5 \text { raters, } \\
\text { follow-up } 1 \\
\text { rater:* } \\
-0.84-0.91 \\
\text { Follow-up, } \\
1 \text { rater: * } \\
-0.62\end{array}$ & & $\begin{array}{l}7 \text { raters: } \\
\text { - mean } 0.76 \\
\text { (range } 0.66-0.82 \text { ) } \\
\text { Threshold: } \\
\text { - enamel range } \\
0.52-0.73 \\
\text { - dentine range } \\
0.52-0.90\end{array}$ & $\begin{array}{l}2 \text { raters: } \\
\text { similar values } \\
-0.67 \\
1 \text { additional } \\
\text { rater: } \\
-0.92\end{array}$ \\
\hline Agreement & $\begin{array}{l}\text { Caries vs healthy } \\
\text { (study IV) } \\
-96 \% \\
\text { all severities: } \\
-94 \% \\
-95 \% * * * * *\end{array}$ & $\begin{array}{l}2 \text { raters } * * * * \\
-100 \%\end{array}$ & $\begin{array}{l}\text { - }>80 \% \\
\text { - enamel range } \\
82-91 \% \\
\text { - dentine range } \\
86-97 \%\end{array}$ & $\begin{array}{l}2 \text { raters: } \\
\text { similar values } \\
-74 \% \\
1 \text { additional } \\
\text { rater: } \\
-94 \%\end{array}$ \\
\hline
\end{tabular}


Table 5. Summary of data and quality of evidence for studies with a reference-standard (caries increment) threshold $>0$ caries lesion for previous caries experience and salivary mutans streptococci used to identify individuals with increased risk of developing coronal caries. Quality of evidence rated as high, moderate, low or very low and reasons for grade of evidence applied according to GRADE (Atkins et al., 2004). $L R+=$ likelihood ratio positive; $L R-=$ likelihood ratio negative (study I).

\begin{tabular}{|c|c|c|c|c|}
\hline \multirow[b]{2}{*}{$\begin{array}{l}\text { Method } \\
\text { (Threshold) }\end{array}$} & \multirow[b]{2}{*}{$\begin{array}{c}\text { Number of } \\
\text { individuals/ } \\
\text { studies }\end{array}$} & \multicolumn{2}{|c|}{ Diagnostic accuracy } & \multirow{2}{*}{$\begin{array}{c}\text { Quality of } \\
\text { Evidence } \\
\text { - Reasons } \\
\text { for lower grade } \\
\text { of evidence }\end{array}$} \\
\hline & & $L R+$ (range) & LR- (range) & \\
\hline $\begin{array}{l}\text { Previous caries } \\
\text { experience } \\
\text { (>0 caries } \\
\text { lesion) }\end{array}$ & $3454 / 11$ & $\begin{array}{c}3.14 \\
(2.06-4.79)\end{array}$ & $\begin{array}{c}0.46 \\
(0.34-0.60)\end{array}$ & $\begin{array}{c}\text { Low } \\
\text { - Limitation of } \\
\text { study quality } \\
\text { - Significant }\end{array}$ \\
\hline $\begin{array}{l}\text { Salivary mutans } \\
\text { streptococci } \\
\text { (>5-105 } \\
\text { CFU/ml) }\end{array}$ & $2,523 / 6$ & $\begin{array}{c}3.32 \\
(2.21-8.11)\end{array}$ & $\begin{array}{c}0.64 \\
(0.33-0.80)\end{array}$ & $\begin{array}{l}\text { inconsistency } \\
\text { - Uncertainty } \\
\text { about evidence }\end{array}$ \\
\hline
\end{tabular}




\section{DISCUSSION}

The burden of dental caries development can be assessed in several ways depending on goals, priorities, assets, and level of aspiration. Global, societal, or individual approaches can be chosen and these can differ enormously. Here, an approach including the individual was chosen, and the increased risk of caries lesion development at surface level was examined. However, caries risk is a difficult concept since judgements have to be based on estimations of probabilities and uncertainties. The assessed probabilities may not be representative for each individual in question, but the uncertainty of this cannot be avoided. Only when we know what we do not know can we begin to improve caries risk assessment and ultimately patient care.

\section{Guidelines}

Previously, the absence of methodological guidelines concerning evaluation of clinical tests was a major impediment since tests are a critical part of the clinical process (Chien \& Khan, 2001). Efforts have been made to, for example, improve formulations of recommendations for research on the effects of treatment (EPICOT+, evidence, population, intervention, comparison, outcome and time) (Brown et al., 2006). Several websites have emerged, such as the Equator Network (http://www.equator-network.org) containing over 350 guidelines. There are also newer webpages under development, such as Penelope research (http://www.peneloperesearch.com). Penelope research allows for manuscripts to be uploaded and, among other things, instantly auto-checked for the use of appropriate guidelines. The GRADE working group stated recently that several BMJ journals joined forces and now demand that authors state the 
appropriate guidelines followed before submission, reinforcing the importance of adequate methods and reporting. Also, the GRADE working group and the Equator Network are active on social media, spreading their message and related literature on the internet. The "new" focus on overall methodological quality and limitations of poor research has the potential to steer the research community towards improvement. Also, agencies such as the Swedish Agency for Health Technology Assessment and Assessment of Social Services and the Cochrane collaboration has increased their visibility, or maybe they currently just draw more attention.

Appropriate guidelines were implemented in study I, II, and IV to underpin the study design and to facilitate transparent reporting. The systematic review (study I) was conducted in accordance with the PRISMA Statement (Moher et al., 2010) and CRDs' recommendations (Centre for Reviews and Dissemination, 2009). In addition, the overall approach of our review complies with the content of the validated AMSTAR-tool (Shea et al., 2007), a measurement tool used to assess the methodological quality of systematic reviews.

Assessment of the study quality of the included studies is an integral part of any systematic review. We defined quality according to Whiting et al. (2011) as "both the risk of bias and applicability of a study; 1) the degree to which estimates of diagnostic accuracy avoided risk of bias, and 2) the extent to which primary studies are applicable to the review's research question". If the results of individual studies are biased and synthesised without any consideration of quality, the results of the review will also be biased (Whiting et al., 2011). Bias occurs if systematic flaws or limitations in the design or conduct of a study distort the results, for example through recruiting an incorrect sample, or using an incorrect index test or reference standard, or carrying out the test in an incorrect manner. This is taken into account by the QUADAS-2 that helps reviewers to make judgements on potential bias and applicability concerns in diagnostic test accuracy studies (Whiting et al., 2011).

In our systematic review, risk of bias was assessed in relation to the problem specification with the aid of a modified QUADAS-2 protocol. To the best of our knowledge, our systematic review was the first in its field implementing the QUADAS-2 protocol, analysing study bias in the assessment of study quality. 
When implementing the QUADAS-2, it is essential to adapt the tool to the specific review question. In our review, the signalling questions were aligned and piloted considering the problem specification and additionally adapted to the actual index test analysed. The sections or "domains" of QUADAS-2, "Index test" and "Reference standard" were weighted to be more important than the domains "Patient selection" and "Flow and timing". This is because the index test and reference standard constitute the foundations of accuracy estimations. Since the caries risk assessment methods dietary variables and oral hygiene measures presented a high risk of bias in the index test domain, these methods were excluded from further analysis.

As proposed by Whiting et al. (2005) either the results of the entire assessment or of key components of the assessment should be taken into account for studies on diagnostic tests. In this respect the QUADAS-2 (Whiting et al., 2011) is a considerable improvement on the previous QUADAS-tool (Whiting et al., 2003) that was originally developed as a tool for quality assessment of studies of diagnostic accuracy. QUADAS-2 allows for a clearer evaluation of the risk of bias (Whiting et al., 2011) as sample selection, index test, and reference standard domains and flow and timing are separately assessed. Moreover, concerns regarding the applicability are judged whether a retrieved study matches the review question or not. However, the concerns regarding applicability are often assessed earlier in the process of publication selection, which was the case in our systematic review and probably in other systematic reviews as well. Our experience is that most publications included in full-text present no concerns regarding applicability if the review question is clearly defined and inclusion and exclusion criteria aligned to the review question. The applicability tells us if the included study matches the review but do not assess the external validity of the included studies since it comes down to the intended implementation of the results.

For studies III and IV, items of STARD-statement (Bossyut et al., 2015; Cohen et al., 2016) were implemented. It contains a checklist of essential elements to be included in diagnostic accuracy studies and was developed to improve the completeness and transparency of reports. STARD was first published in 2003 (Bossyut et al., 2003) and updated in 2015 (Bossyut et al., 2015; Cohen et al., 2016). 
Since 2003, evaluations have found small but statistically significant improvements in the reporting of diagnostic accuracy studies. But the situation remains far from ideal (Korevaar et al., 2015).

In the strive towards transparent and complete reporting, enabling validation and critical appraisal in studies on rater performance, other guidelines are at hand. One such set of guidelines are the GRRAS guidelines (Kottner et al., 2011a), which were implemented in study II. To the best of our knowledge, these guidelines have not previously been applied in studies published in dental journals. In both GRRAS and STARD the importance of a full description of sample and methods is emphasised. It is also explicit that statistical uncertainty, such as confidence intervals, should be included in estimates of agreement and accuracy respectively. In line with GRRAS, we reported agreement both as a percentage of agreement and as kappa statistics with CI to "allow the reader to get a detailed impression of the degree of the reliability and agreement" (Kottner et al., 2011a).

The recently developed TRIPOD (The Transparent Reporting of a multivariable prediction model for Individual Prognosis or Diagnosis) initiative presents recommendations for the reporting of studies developing, validating, or updating prediction models, whether for diagnostic or prognostic purpose (Collins et al., 2015). It differs from STARD as it is developed to be applicable to any method of prediction but mainly for multivariate models, whereas STARD (Bossyut et al., 2015; Cohen et al., 2016) is costumed towards diagnostic test accuracy studies.

\section{Methodological Considerations}

In general, there was heterogeneity in study design and methodology between included studies of our systemic review. Sources of bias were identified in all included studies, which is in line with previous systematic reviews on caries risk assessment (Mejàre et al., 2015; Tellez et al., 2013a). As found by Ioannidis (2014), in medical research there is a lack of written protocols and poor documentation. To be able to replicate a study, a thorough description of the methods used is a necessity. Our assessment of study quality was hindered by a lack of clear reporting on pre-specification of threshold values, on examiner reliability and reporting concerning whether or not examiners were 
blinded to previous results in follow-up examinations. Deficiencies in the reporting of diagnostic accuracy studies have been identified in an increasing number of studies (Cohen et al., 2016; Collins et al., 2015; Korevaar et al. 2015) as well as potential sources of bias (Lijmer et al. 1999; Whiting et al. 2013). In our experience, the QUADAS-2 protocol with four domains facilitated our evaluation of the research methodology of included studies in our systematic review. Therefore, I will apply the four domains, (i) patient selection, (ii) index test, (iii) reference standard, and (iv) flow and timing when discussing methodological considerations.

\section{Patient Selection}

A study ideally should enroll a consecutive or random sample of eligible patients to prevent the potential for bias. One common error according to Kim et al. (2011) is when a selected sample is described as being random when it is not. Self-selected samples are often known to be biased and can lead to misleading results (Kim et al., 2011). Studies that make inappropriate exclusions may result in overestimation of diagnostic accuracy. Concerns about applicability according to QUADAS-2 may exist if patients included in the study differ from those targeted by the research question in terms of severity of the target condition, demographic features, presence of differential diagnosis, setting of the study, and previous testing protocols. The consecutive enrolment of adolescents in study IV resulted in few individuals with numerous caries lesions, few individuals with dentinal lesions, and none with dentinal lesions exceeding $1 / 3$ of dentine. Only $12 \%$ of the included adolescents exhibited dentinal lesions, which is less than in a Swedish study of adolescents which showed a $22 \%$ prevalence of unrestored dentinal lesions (Ridell et al., 2008). Further, the Swedish National Board of Health and Welfare (2013) found $34 \%$ of 12 -year-olds exhibiting dentine lesions.

Caries prevalence, including enamel lesions, in adolescents examined in study IV is difficult to compare with epidemiological data since these data usually are based on clinical examinations and only cavitated caries lesions (Hintze \& Wenzel, 1993). The representability of the samples in $\mathrm{WHO}$ data on caries prevalence is scarce (Patel et al. 2016). For example, the Croatian DMFT score is said to be derived from two schools in Zagreb (Dukic et al., 
2011). Furthermore, examples on low representability are given for additional countries. Thus, the generalisability of results for each county may be questionable.

The two different samples included in study IV facilitated a comparison and demonstrated that patient characteristics differ between clinics. This may contribute to the generalisability of our results. On the other hand, sample size was limited, which is compromising since extreme outcomes, both high and low, affect results on overall estimates on diagnostic accuracy to a greater extent in smaller samples.

\section{Index Tests}

The index test should be described in sufficient details to allow for other researchers to replicate a study or to allow readers to assess the feasibility of the test. For caries risk assessment, different index tests have been used, as reported in our systematic review (study I). We excluded studies of some tests, as there were potential areas of concerns regarding the description of and the execution of these tests. The index tests previous caries experience and dentist's risk assessments are based on the detection of caries lesions, which are examined by visual and/or tactile examination and radiography. In the following I will discuss these examination methods and caries classification systems, which were implemented in studies I and IV. Additionally, I will reflect on microbiological tests, which were used in several included studies (I, III, IV).

\section{Visual/Tactile Examination and Bitewing Radiography}

The current view of the caries diseases process is a slow progress under an intact outer occlusal enamel. This contributes to the difficulties in detecting occlusal lesions by visual examination alone (Kidd et al., 1992; Wenzel et al., 1991). Wenzel et al. (1991) found the true positive detection rate for occlusal lesions to be double for radiography as compared to visual inspection, without increasing the number false positive. We do not know to what extent the GDPs used the bitewing images as a supplement to their visual and tactile examination. If so, they may have identified more lesions than with the visual and tactile method alone (study IV). Even if this could have improved accuracy of caries detection and so increased the number 
of decayed surfaces (and DMFS), this was neither the instruction nor the task. The caries lesion distribution in the sample of one clinic (clinic S) does not strongly imply such a bias, since the occurrence of occlusal/buccal and lingual lesions was low. In the other clinic (clinic A), the occlusal and buccal/lingual surfaces constituted about half of the decayed surfaces. This discrepancy in disease distribution between the clinics cannot be explained for; this was merely the characteristics of the sample given in the specific catchment area. The follow-up examination at 2 years of the sample at clinic $S$, demonstrate the same trend with a low caries frequency of occlusal and buccal/ lingual caries lesions. The trend in clinic A is maintained with a larger proportion of such lesions after 2-year follow-up.

For approximal caries lesions, accuracy of visual and tactile examination has been shown to be lower than that of visual/tactile examination together with radiography (SBU, 2007). Bitewing radiography was implemented in study IV, enhancing the internal and external validity of the results. The validity of the studies included in study I, which based the index test previous caries experience on visual or visual/tactile examination alone, may be questionable. The validity is of course dependent on the intended role and placement of the index test or the clinical context. Therefore, visual examination alone as a basis for previous caries experience may be appropriate for research of large populations and as long as the lower index test thresholds of few lesions is accounted for. The consequence may be many false negative diagnoses and lower accuracy estimates expressed as sensitivity and LR+.

Various diagnostic classification systems were implemented in the studies on caries risk assessment included in our review. The systems used different numbers of scoring categories and caries severity was recorded differently. The content of the categories, thus the lesion characteristics subsequently differed. The lack of consistency among the criteria systems made it difficult to synthesise the results of the included studies. In the WHO caries classification criteria ( $4^{\text {th }}$ ed.) there is a clear threshold value for caries, i.e. a cavitated dentinal lesion. This will lead to a serious underestimation of the prevalence and extent of dental caries in the included populations (Baelum et al., 2007). 
The GDPs in clinic A and S (study IV) assessed all surfaces except the approximal surfaces using the ICDAS visual and tactile caries classification system, which is often reported to be used in clinical trials on caries (Ismail et al., 2007). Each tooth surface was examined clinically and classified using a six-point-scale. This system provides detailed exclusion criteria of non-carious lesions and has shown substantial (or higher) inter- and intra-rater agreement in terms of kappa values. ICDAS has been applied in studies on risk indicators for dental caries and in evaluating a new scoring system (Ismail et al., 2011; Ismail et al., 2008), but there are complicating factors seen in longitudinal studies using ICDAS (Ismail et al., 2011).

\section{Microbiological Tests}

In study I we concluded that microbiological tests in caries risk assessment were seldom described in a way that permitted replication. Any method, microbiological tests included, must be scrutinised to the same extent as other diagnostic and/or predictive methods. The limitations in reporting may reflect the scientific traditions in this field that usually do not embrace measures of, for example, reliability of methods. The use of a strict preconceived protocol might not be feasible for some exploratory research, but nonetheless researchers should rigorously document the sequence of decisions and findings made in the course of the study, and reasons for those decisions (Ioannidis et al., 2014).

There are several factors that may influence the results of microbiological tests (Griner et al., 1981), such as the acid tolerance test analysed in studies III and IV. First, the sampling requires an adequate amount of dental biofilm, allowing for proper assessment of acid tolerance. As the adolescents were not instructed to refrain from oral hygiene habits, the amount of biofilm may be limited from some of the tooth surfaces in the adolescents. Secondly, a successful transport of samples depends, among other things, on keeping the sample moist. If the environment is too dry the sample might dry out and this affects the viability of the bacteria. The consequence would be an assessment of the acid tolerance as low, which may result in false negative diagnoses. However, Neilands et al. (2012) performed similar transport of a large number of samples, with sustained viability. Furthermore, in the laboratory, optimal $\mathrm{pH}$-value for acid shock of 
the sample was previously established (Neilands et al., 2012). Since it is not validated, this is a comprising fact. In summary, sampling technique, transport difficulties or chosen optimal $\mathrm{pH}$ for acid shock of dental biofilm samples might have influenced the results of studies III and IV i.e. the scores and subsequent the caries risk assessment.

\section{Reference Standard - The Not So Golden Standard}

A reference standard cannot be ambiguous and cannot itself be the test and reference. An imperfect reference standard can lead to either positive or negative bias (Walter, 2012). There is evidence from several medical studies that the choice of reference standard affected the results of diagnostic accuracy estimates (Whiting et al., 2013). This has been shown also in radiographic studies of caries lesion detection (Hintze \& Wenzel 2002; Wenzel \& Hintze 1999b; Wenzel, 1993). The criteria for the reference standard are to: "(1) be reproducible, (2) reflect the patho-anatomical appearance of the disease, and (3) be independent of the diagnostic method under evaluation" as stated by Wenzel and Hintze (1999b).

Poor reporting on the definition of the reference standard and how it was examined resulted in "unclear risk of bias" for the domain reference standard for most studies included in our review. In only one study, the reference standard was clearly described "the caries increment was calculated by surface subtracting the baseline dmfs or DMFS score from the last available dmfs or DMFS score, without considering reversals" (Sánchez-Pérez et al., 2009). Two studies (Pienihäkkinen et al., 2004; Sánchez-Pérez et al., 2009) provided information that the reference standard was interpreted without knowledge of index test results, which is an important item when assessing risk of bias. In study IV, one potential for bias was that the GDPs performed the 1-year follow-up examinations with access to previous records of the adolescents. On the other hand, it adds to the generalisability of results, as it is the common procedure at patients' regular dental check-up appointments in Swedish general dental practice.

If the purpose of caries risk assessment is to apply interceptive prevention, it was remarkable that enamel lesions were included in few studies included in our review. One can argue that the validity of predictive studies with caries lesion development as reference standard is reduced if enamel lesions are not included in the criteria. 
As more focus and effort is put to early detection and prevention of lesions, images are vital to reveal enamel, which comprised the major part of lesions in adolescents in study IV. To improve the validity of studies of caries risk assessment and be able to evaluate preventive measures, we found that a classifications system for caries lesion progression including enamel lesions is needed. The classification system, which is based on bitewing radiography, was developed and piloted in study II.

The classification system take advantage of by side-by-side comparison of approximal surfaces in bitewing radiographs to detect small hard tissue changes. According to our collaborators working in the public dental service, such a side-by-side comparison of bitewing radiographs is the standard practise at their follow-up examinations of patients. This practice is in line with perceived recommendations for operative intervention threshold, which is partly determined by an observed lesion progression in radiographs.

Quality controls of the radiographic equipment and resulting images as performed in studies II and IV are prerequisites for an accurate assessment of caries lesions, in particular caries lesion progression. A high and comparable quality of baseline and follow-up images creates conditions for detecting small differences and changes in radiolucency. This is especially important for the radiographic examination of approximal surfaces, where progression of caries lesions is common. The images were performed in general dental clinics in an everyday clinical environment without any standardisation of exposure times or projection geometry (studies II and IV). This adds to the generalisability of results but may increase the number of images with, for example, overlapping surfaces as compared to studies optimising these features.

"Estimates of test accuracy are based on the assumption that the reference standard is $100 \%$ sensitive and specific, disagreements between the reference standard and the index test are assumed to result from incorrect classification by the index test" (from QUADAS-2, Whiting et al., 2011). In studies of caries lesion detection, histology is often the reference standard of choice, as it is deemed to fulfil the three criteria presented by Wenzel and Hintze (1999b). Though, the reference standard itself needs to be assessed whether or not it actually is a trustworthy indicator of prescience of disease (Hintze et al., 1995). There are different approaches to how 
to section a tooth. Hintze and Wenzel (2003) found significantly more caries lesions in serially sectioned than hemi-sectioned teeth, irrespective of caries lesion depth. Of surfaces assessed as sound in the hemi-sectioned teeth, $35 \%$ presented caries lesions when they were recorded following serial sectioning. This seems logical and is probably not due to an increased false positive rate. When storing specimen, it is important to recognise the deleterious impact on the radiographic optical density that has been shown to be the outcome of prolonged storage in formalin. Radiographic optical density of specimens stored in formalin decreased with time and could alter the quality of radiographic images (Fonseca et al., 2008).

There are difficulties to establish a reference standard for caries lesion progression. When the three criteria for a reference standard cannot be achieved, alternative approaches have been applied in previous studies of diagnostic accuracy. Consensus assessment of several observers or experts as an expression of the reference standard has been used. However, such an approach does not fulfill two of the criteria for a reference standard. This practice is discouraged since it can give rise to high and incorrect accuracy estimates (Hintze \& Wenzel, 2002; Wenzel \& Hintze, 1999a). Results can also be unpredictable and have a limited generalisability as they depend on the observer or experts chosen (Wenzel \& Hintze, 1999a). Another alternative approach to a reference standard would be artificial lesion progression, using a technique for lesions with increasing size, as performed in studies on periapical bone tissue in situ (Kullendorf et al., 1988). It may however, be difficult to simulate caries lesion progression as its appearance may differ in different individuals. Furthermore, initial approximal lesions in human premolars and molars may be found not only in one typical site but in three sites: within the contact area, in the subcontact area, and in the cervical areas (Arnold et al., 2000). Therefore, it may be difficult to simulate "natural" caries lesions or lesion progression as its appearance differs in different individuals and sites.

\section{Flow and Timing}

Flow is about whether all patients who were recruited into the study were included in the analysis. There is a potential bias if the number of patients enrolled differs from the number of patients included in 
the $2 \times 2$ tables of results. The patients lost to follow-up may differ systematically from those who remain, which may inflate diagnostic accuracy. This was the case in study IV where the withdrawals in both clinics presented higher caries prevalence and different caries characteristics at baseline. The term used for this is verification bias, which in study IV probably contributed to the estimated accuracy of analysed tests being underestimated when compared to ideal circumstances. To judge this bias, a flow diagram that provide information about the recruited and withdrawals is recommended in QUADAS-2 for studies included in systematic review as well as by the STARD statement for studies of diagnostic accuracy. Additionally, the flow diagram should contain an analysis of the reasons for withdrawals, which was seldom presented in studies included in our review.

Timing in many studies of diagnostic tests refers to the time between the index test and reference standard. Results of the index test and reference standard are often collected for the same patients at the same time. Conversely, a reference standard that involves follow-up may require a minimum follow-up period to assess whether a progression is present. What is acceptable as follow-up time is dependent on the patient sample and outcomes. A follow-up time of 1 year was considered reasonable for the primary dentition, as the progression rate is fast (Grindefjord et al. 1995). However, in adolescents the short follow-up time in study IV is a compromising fact as the progression of carious lesions has been shown to be slow in general (Mejàre et al., 2004). The median survival time of approximal lesions located just beyond the enamel-dentin border until they had progressed to the outer half of the dentin was 3.1 years in a population aged 12-21 years (Mejàre et al., 1999). The survival time was only 1 year for $10 \%$ of the dentine lesions. This underlines the importance of detecting dentin lesions, particularly in young teenagers, since the rate of lesion progression was highest from the age of 12 to 16 (Mejàre et al., 2004).

When a delay occurs between performing the index test and the reference standard, as in predictive and prognostic studies, interventions may be started after doing the index test. If the interventions introduced are successful, the reference standard in analysed studies, i.e. caries lesion progression, will be influenced. As 
stated by Hänsel Petersson et al. (2002), it is important to recognise that past caries experience is the effect and not the cause of caries disease, so this variable loses its predictive ability if successful interventions are introduced and risk factors are removed. A detailed description of the interventions is therefore helpful to understand the applicability of the study findings. In study IV, the intervention is compromising since about half of the included individuals underwent an intervention with fluoridated milk, which may affect caries progression.

\section{Diagnostic Accuracy \\ General Reflections}

Data on diagnostic accuracy must be properly processed and analysed in a technically sound and meaningful way to uncover the hidden clues. We presented diagnostic accuracy as several estimates and in appending tables in line with many medical studies of diagnostic studies to facilitate comparisons with results of other studies. We considered the likelihood ratios of special interest as they have the advantage of incorporating all four cells of the $2 \times 2$ table in contrast to sensitivity, specificity, and predictive values, which make use of only two cells. Likelihood ratios are considered to be more clinically meaningful than other measures (Chien \& Khan 2001; Grimes \& Schulz 2005; Honest $\&$ Khan 2002). Yet no study included in study I presented this accuracy estimates. Deeks (2001) claims that the ORs and ROC-curves are considered by some as the most statistically valid for combining test results when there is heterogeneity between studies. For the clinician, though, these measures cannot compute the probability of disease associated with specific test outcomes (Deeks, 2001). Irwig et al. (1995) argues for a cautious use of new metaanalytic techniques and for the assessment of study quality and how quality or test and patient characteristics determine test accuracy. This is in line with our considerations in study IV.

There is a danger of misinterpretation of results if not taking into account wide confidence intervals, which indicate considerable uncertainty (Altman \& Bland, 1995). No study included in study I described the statistical uncertainty, as recommended by STARD (Bossyut et al., 2015). The underuse of confidence intervals in studies is in line with the results of a review of papers published in dental 
journals (Kim et al., 2011). However, we calculated the confidence intervals of included studies and judged it as high. The results from study IV with different thresholds produced results with wide confidence intervals due to few affected individuals.

\section{Some Factors Influencing Accuracy Estimates}

When creating a threshold, the index test and reference standard are dichotomised i.e. divided into two groups of health and disease or disease and more severe disease.

The choice of threshold is not a statistical decision but takes into account what is clinically meaningful and is associated with the relative costs of false positive and false negative results (Altman, 1991). However, in dichotomising one loses information (Irwig et al., 1995; Jaeschke et al., 1994; Habbema et al., 2002; Streiner et al., 2002). Factors relating to tests included in caries risk assessment are seldom natural dichotomies; therefore, we need to artificially dichotomise them (Hausen et al., 1997). Streiner (2002) argues that one pays a penalty in terms of power or sample size, with the consequent risk of failing to detect real differences because of this loss of information. Similar criticism towards dichotomisation was stated by Bratthall and Hänsel-Peterson (2005), who considered sensitivity and specificity measures for a multifactorial disease like caries has many times failed and set back proper use of cariesrelated information and tests for many years. Estimates calculated in diagnostic accuracy studies differ, however, from the presentation in studies of risk factors. One alternative to dichotomisation is the regression analysis, also mentioned by Streiner (2002). Examples of two excluded studies from study I were Beck et al. (1992) and Disney et al. (1992) from the North Carolina caries risk assessment study with large samples performing regression analysis. Regression models are widely used in medicine to analyse prognostic studies, where the aim is to quantify the risk of a future event (Royston and Altman, 2010). The models in such analyses are generally multivariable, i.e., several explanatory variables are required to predict the outcome acceptably well (Royston and Altman, 2010). However, regression analysis has also been criticised. Amongst other things, criticism has focused on the assumption of the relationships of risk factors between the variables studied to be linear (Keynes, 2015). 
Add-on tests (extra tests after the "ordinary test" have been applied) can increase the sensitivity, possibly at the expense of specificity. Alternatively, add-on tests may be used to limit the number of false positives (Bossyut et al., 2006). This is contradictory to a study of periapical bone lesions by Åkerblom et al. (1988) where add-on tests increased the false positive. This approach was not evaluated for the tests in study IV.

Literature has shown that the sensitivity and specificity may vary with disease prevalence (Feinstein 2017; Lachs et al., 1992; Mulherin and Miller 2002; Tellez et al., 2013a; Whiting et al., 2013). These effects are not always specific; higher prevalence does not systematically lead to either higher or lower sensitivity or specificity but they will vary with clinical and patient characteristics, disease severity, and details of test performance (Hlatky et al., 1984; Mulherin \& Miller 2002) as well as shortcomings in study design (Rutjes et al., 2006). This is known as the clinical variability as defined by Leeflang et al. (2009). The decline in caries prevalence in the last decades will theoretically lead to a fall in the positive predictive value of a diagnostic test. This means that the lower the disease prevalence, the less trustworthy a positive outcome of the test may be (Gröndahl, 1994). Thus, when evaluating the results of a caries risk assessment method it is of the utmost importance to take into consideration the threshold levels that have been used (Hausen, 1997; Wenzel \& Hintze 1999a).

For example, accuracy estimates on previous caries experience varied across studies both due to the age of the sample population and different thresholds in study I. Accuracy also varied within a sample due to different thresholds, exemplified by Kingman et al. (1988), in study I and in study IV since the ranges for different accuracy estimates were wide for the three index tests for a sample within the same clinic. The threshold effect is explicit in study IV, with the same caries prevalence at 1 year and threshold values at clinic $A$ and $S$ the accuracy estimates differ (as do the DFS). This effect can be ascribed to the variation in disease spectrum between clinics, which includes the caries lesion severity at baseline as well as the progression of lesions. The explanation for the higher sensitivity is probably that when caries prevalence increases, the spectrum of disease also changes so that the proportion of more advanced (and more easily detected) caries lesions increases. The reason for the decreased specificity is 
not as obvious but could be that the corresponding proportion of false positives decreases.

Thus, the thresholds of the index tests and of the reference standard as well as the sample characteristics influenced the estimates of diagnostic accuracy in study IV. In general, higher thresholds for index tests previous caries experience and acid tolerance produced higher accuracy estimates and the highest for score $\geq 4$ or DMFS $\geq 4$. Relatively few individuals in these groups lead to a low prevalence of positive individuals at baseline of these "severe" cases. They were subsequently easier to identify at follow-up with a smaller proportion false negative. Since many individuals are negative at follow-up, this must be interpreted, as signifying that an identification of a negative individual will almost always be true.

After developing a prediction model, it is recommended that one evaluates the method performance in other samples (Altman et al., 2009; Collins et al., 2015; Moons et al., 2012). Prediction models can be expected to perform optimistically in the data sample from which they are developed compared with the performance found in new but comparable individuals (overfitting) (Moons et al., 2012). Furthermore, Whiting et al. (2013) have produced evidence that case-control studies overestimate both sensitivity and specificity and that disease prevalence and severity influence estimates in diagnostic accuracy studies.

Therefore, it seems challenging to design a universal method that is applicable to all patients and rather methods can only be used in the context in which they work depending on prevalence but also on disease spectrum. In general, the accuracy of diagnostic methods varies with disease prevalence and the disease spectrum. Therefore, the generalisability is often limited to similar populations, which decreases the external validity for given methods.

\section{Previous Caries Experience}

In general, reviews on studies of caries risk assessment methods show scattered results on sensitivity for previous caries experience (Mejàre et al., 2014; Schwendicke et al., 2015) which is in line with the results from study I. Estimates on specificity were generally higher, suggesting that the identification of individuals with "low risk" of developing caries is more certain (study I). 
Previous caries experience did not stand alone as the best method but displayed equal accuracy values expressed as LRs as the index test mutans streptococci from saliva (study I). Pooled estimates of LR $+\geq 5$ or LR- $\leq 0.2$ that is judged to provide strong diagnostic yield (Deeks, 2001,) were achieved for some thresholds (study I, IV).

\section{Dentists' Risk Assessments}

Beside previous caries experience, clinicians' intuition or "gut feeling" is claimed to be accurate, even more accurate, for caries risk assessment than current diagnostic technologies. Clinicians' intuition is, however, not easily quantifiable for the validation of this criterion and this diagnostic strategy is not well supported by evidence in health care. Dentists' risk assessments, which were evaluated in study IV, seem at first glance to take clinicians' intuition into account. However, the GDPs were not free to score according to their intuition as high and low risk was based on the definition applied in the public dental health of one county. As the definition includes previous caries experience of both primary and permanent teeth, it was remarkable that accuracy estimates were somewhat lower than those of the index test previous caries experience.

\section{Microbiological Tests}

The ecological plaque hypothesis changed our perception of the cause of oral diseases are caused with an emphasis on physiological adaptation to environmental conditions, rather than on specific bacteria. New predictive biomarkers should consequently adhere to this assumption, but the literature on genotypic expression is still numerous. Some claim that new methods should be assessed on the value they add to existing prediction models or predictors, rather than simply being tested on their predictive ability alone (Moons et al., 2015). However, the added value of the new microbiological method would be difficult to distinguish from the contribution of other methods in a multifactorial model. To decide on additive value of the new method one needs to test the old and new model simultaneously in the exact same setting. Microbiological methods are often compromised by methodological deficiencies (study I), presumed additional examination time, and monetary means lost in the process of sampling and analysis. 
There are several possible reasons for sensitivity being lower than specificity seen for tests that rely on the count of specific bacterial species (study I). For example, it is well known that dental plaque harbours other streptococci that are capable of exhibiting an acid tolerant phenotype (e.g. Streptococcus gordonii and Streptococcus oralis), which would lead to a false negative result in this type of test (de Soet et al., 2000). On the other hand, the presence of non-aciduric mutans streptococci in the sample would give rise to false positive results. In evaluating acid tolerance of dental biofilm in caries risk assessment, one way of possibly achieving higher accuracy would be to sample biofilm at several occasions to obtain the bigger picture of what the actual score of the individual is and not the score for that particular period.

For a caries disease, it seems valid to concentrate on the lowest abstraction level where the disease is found, i.e. the actual site with disease and the local risk factors associated with disease development at that site. Acid tolerance of dental biofilm is suggested to be such a "factor". Considering the current state of knowledge on caries aetiology, it seems fair to argue that acid tolerance is a local environmental feature essential for disease development. There are difficulties though since this test is not binary, at least not yet. In caries risk assessment, difficulties arise on dichotomisation of the test 5 score scale. To meet this challenge and to visualise the possible influence of different thresholds on accuracy estimates, data on multiple thresholds were assessed (study IV). This is in line with the Cochrane handbook that clarifies the usefulness of several selected threshold values (Macaskill, 2010)

\section{Reliability and Agreement}

It is argued that there are important differences between agreement and reliability (Streiner \& Norman, 2008; de Vet et al., 2006). Kottner and Streiner (2011) suggest that much of the confusion surrounding reliability and agreement estimation was and is caused by conceptual ambiguities. This is in line with our findings from the scientific literature on reliability and agreement with different definitions of concepts as well as different ways of expressing these in terms of statistical data. In addition, some literature deals with the concept of reproducibility, also with various definitions. 
It is vital to define these concepts, this enabling comparison of studies, evaluation of methods, and to facilitate the appropriate statistical approach.

The definitions aside, it comes down to the actual application of data. de Vet et al. (2013) suggest that clinicians are interested in agreement and pose questions such as "Is my diagnosis in agreement with that of my colleagues?" i.e. inter-rater agreement, and "Would I obtain the same result if I repeated the assessment?", i.e. intrarater agreement. Therefore, both kappa and agreement in percentage was presented in study II. Because statistical measures were often developed within different disciplines, no single approach can be regarded as standard or be appointed as the "best" method. The choice of method should be dependent on the aim and design of the individual study (Kottner et al., 2011a).

Rater variation is said to be the Achilles' heel of diagnostic imaging (Robinson, 1997). The index test and the reference standard are seldom perfect with respect to validity and reproducibility (studies I, IV). The reproducibility varies, and limited reproducibility adversely affects diagnostic accuracy since it is one determinant of prevalence and severity. Therefore, the test reproducibility and methods for calculating reproducibility should be presented in studies of test accuracy. However, for the clinical examination performed by GDPs in clinic A and S (study IV) rater agreement was never established.

As we aimed to imitate clinical practice, no effort was made to calibrate the observers for study II. The prevalence of caries lesions in the sample of study II were higher than for a general population of adolescents in Sweden. This approach is usually recommended for agreement studies but the generalisability can be questioned (SBU, 2007). Observers did not receive prior information on the prevalence of lesions since an expectation to find a high prevalence has been proven to lead to more positive findings (Gröndahl, 1979).

\section{Risk}

As risk is an often-used term in dental research I find some reflections on risk justified. Although risk is commonly mentioned in oral health care and public dental service guidelines, the concept of risk has not been thoroughly defined, maybe because risk is a vague concept. There is more to the concept of risk than outcome risk, regardless 
of how it is defined and there is not one but numerous definitions of the term risk (Brinck et al., 2005).

The general view is that risk has something to do with the outcomes of our actions - with their character, seriousness, and likelihood. Thus, risk can be taken to mean something like "an outcome below an aspiration point", "the probability of a negative outcome", "the frequency with which a negative outcome occurs", "negative utility", information about "objective" probabilities. We do not say that we are at risk if all possible outcomes are good, favourable, or make us happy. We are at risk or we take a risk when, at least one, of the possible outcomes is negative, harmful or below our level of aspiration. In addition, if the outcome is certain there is no risk. If I know that my only remaining molar will be lost, that is it. I can like it or not, but there is no risk involved. In other words, risk means that there is an element of uncertainty and that at least one possible outcome is negative.

When we talk about risk we add a value statement to our probability judgment. There are also different definitions of risk factors, risk indicators, risk markers, and risk assessment in the scientific literature dealing with risk and oral diseases. Since the 1980s there has been an increase in the publications found when using the search terms "risk assessment" and "dental caries" in PubMed database. There are 3 publications found indexed during 1980s, 106 during 1990s, and 408 during the twenty-first century (accessed March 2017). This could be due to a change in the use of the wording in title and/or abstract and/or a change in the indexing systems of PubMed database. It could also reflect an increased interest in this field. I analysed 17 carefully selected publications on caries risk assessment regarding their definition of the risk concept and though the publications elucidated risk of caries, none defined the concept of risk itself.

During the past 40 years, individual risk estimation has been broadly accepted in medicine (Stern, 2012). For this thesis, risk was defined according to the US National Library of Medicine (2017) as "the probability that an event will occur. It encompasses a variety of measures of the probability of a generally unfavourable outcome". In this context probability means an individual or personal probability. If the decision-maker can put an objective measure on this uncertainty, we can assess risk. In the ideal situation, there is an objective probability that the event will occur. 
According to the literature, a person's risk of caries varies with time since many "risk factors" are changeable (Selwitz et al., 2007). Stern (2012) gives a slightly different perspective and states that “... patients don't have an "individual risk" or unique probability of an outcome...risks are conditional probabilities and differ as the risk factors included differ, at times substantially. This lack of reliability is an inherent limitation and is not resolved by including additional risk factors". This seems fair! There are numerous difficulties in assessing risk and subsequently it is impossible to endorse any specific kind of caries risk assessment method and implementing it onto an individual patient. The methods cannot be scientifically evaluated for each and every individual patient at every point in time. Major generalisations are (unfortunately) required.

Referring back to the statement of Stern (2012), given the knowledge and information the clinician has, the probability that caries lesion will cause any serious problem for the patient is probably very low but we do not know the "individual risk". However, this particular patient, a middle-aged, bewildered and candy-addicted man, is a different story. The known frequencies have to be adjusted; a subjective probability assessment is required for the factors viable at this point of time. However, we lack the tools to do so in an accurate manner.

Also, oral health care involves more than one decision-maker. We have the patient and the clinician, but also society at large. It is obvious that the patient's, the clinician's and our society's levels of aspiration may differ. They have different knowledge, information, and experience influencing their assessments. In addition, the level of aspiration is affected by the measurability of the event, in this example, dental caries. If we are able to measure the slightest molecular decrease in mineral content of enamel, would we then escalate our level of aspiration or would we settle for less?

From our systematic review, it seems that the aspiration level is rather high as the threshold value for new caries lesion includes one lesion. Is there more or less a zero tolerance towards dental caries development in oral health care for populations? The level of aspiration seems very high but it also has to be feasible. One can assume that this level of aspiration collides with many individuals' view on oral health. The middle-aged, bewildered, and candy-addicted man may find it more problematic refraining from sugary pastries and brushing his teeth 
than getting one cavity per year. This supposition problematises the difficulties in risk-communication between caregiver and patient. It also elucidates the importance of understanding the concept of risk when communicating with different individuals as well as when communicating within the scientific community.

By now the general knowledge is that clinical methods for caries risk assessment display inherent deficiencies and evidence of caries risk assessment methods and several preventive measures are scarce. This discrepancy between level of aspiration and lack of tools illustrates a major challenge in the struggle to predict and limit development of dental caries lesions in populations.

\section{Transparency and Research Waste}

The Lancet REWARD (REduce research Waste And Reward Diligence) campaign has encouraged researchers to examine how they work and make efforts to reduce waste and maximise efficiency (Ivers \& Grimshaw, 2016). The campaign calls for more, but above all, well conducted research within field after field in health research. When it comes to research methodology, guidelines seem to be more frequently implemented today than previously in dental research.

Although replication of previous research is a core principle of science, at some point, duplicative investigations may contribute little additional value (Ioannidis, 2014). Thus, to reach high evidence for any intervention, diagnostic, predictive, preventive or treatment, consistent results across several high-quality studies are required. Health technology assessments and other high quality systematic reviews in specific topics, therefore bears repeating.

Our systematic review partly overlapped with the SBU report no 188 (2007) and the study by Mejàre et al. (2014), but the problem specification, criteria, and analyses were different. In spite of this, we argue that some results of the systematic reviews are comparable and may contribute to evidence. Our results tell us that there is no overwhelming increase in well-conducted studies of caries risk assessment between 2007 and 2015. More remarkably, with deep analyses of methodological limitations in our review, some results were not in conjunction with the results of the two other reviews.

It is not viable to perform an overall evaluation of the recent research that emerge regarding methods on caries risk assessment. The question is, does the scientific community respond by shifting 
focus? Or is it perhaps easier to continue with ongoing projects based on old ideas that easily produces numerous publications?

Previous caries experience has for a long time been considered to be the best method. It shows that an individual has had the capacity to develop caries lesions, indicating that the factors required for caries development have been present. The use of previous caries experience as a predictor for new lesions builds upon the expectation that these conditions will be maintained over time and new caries lesions will eventually develop. However, this is not necessarily the case since factors such as dietary habits, oral hygiene routines, and fluoride exposure change over time.

A shift in focus could be to identify a biomarker that is directly related to the demineralisation process. The acid tolerance test attempts to measure a property of caries inducing biofilms as an indicator of their acid producing potential. According to the biomarker development pipeline, study III lies in the verification phase to verify the acid tolerance test, i.e. to begin to assess the specificity of the candidate and its reliability (Rifai et al., 2006). In contrast to previous caries experience, the focus of the method is not retrospective but on prevailing conditions and the method could be prospective. Study IV is a pilot attempt to assess the diagnostic accuracy estimates of the acid tolerance test. We hope that this will represent a shift in focus towards a more biological approach to diagnostic methods for caries prediction.

\section{Evidence - or Rather the Absence of Evidence}

Absence of evidence is not evidence of absence (Alderson 2004; Altman \& Bland, 1995).

"More research is needed" is a well-known conclusion that fits most systematic reviews. Brown et al. (2006) stated that review authors need to be more specific to create helpful research recommendations. Failing to do so lead to lost opportunities to identify knowledge gaps and the potential value of these recommendations is lost (Brown et al., 2006). Chalmers (2010) stated that the reason for the reviews not ending with clear instructions, leaving readers dissatisfied, is that there is simply inadequate research evidence to provide reliable information in order to guide the choices people must make. "Evidence-based medicine offers little help in the many grey zones 
of practice where the evidence about risk benefit ratios of competing clinical options is incomplete or contradictory" (Naylor, 1995). The limits to medical evidence continue to limit the influence of evidence-based medicine (Naylor, 1995). It is difficult to endorse uncertainties. However, researchers either accept the uncertainties or reduce them by additional research (Chalmers, 2010).

Taking this advice and elaborating on the conclusions from study I, which stated an "urgent need to improve study design, test technology, and particularly reporting of studies on caries risk assessment", a suggestion would be i) for primary studies to adhere to appropriate guidelines such as STARD (Bossyut et al., 2015) and QUADAS-2 (Whiting et al., 2011). Doing so would lead to an enormous improvement in design of the trial and reporting. Furthermore, ii) the index tests used, in particular those based on microbiota, needs to be scrutinised like any other diagnostic method and described accordingly. The upper boundary for detection levels of microbiota, appropriate validation of growth of species as well as parameters such as rater agreement should be included. Additionally, iii) the external validity of results needs to be considered. Many primary studies included in our review failed to include bitewing radiography to assess enamel lesions, even though the results of these studies were probably intended to be implemented in clinical environments.

Following the philosophy of interceptive prevention, accurate detection of enamel lesions is important for the reduction of progression of lesions. Though, the need for bitewing images may vary depending on local resources and the intent of the investigation in question. Further, iv) it would be crucial to include experts from different fields when designing and performing a primary study, avoiding the pitfalls described in our review. Knowledge of diagnostic and predictive methods, research methodology as well as the actual condition/disease researched, in this case caries, is a prerequisite for successful planning and execution of studies. Diagnostic test accuracy studies are challenging to perform and numerous possible sources of bias have been acknowledged (Whiting et al., 2004).

"The (Cochrane) Collaboration has made it clear that research evidence isn't everything. Other factors need to be taken into account when people decide what action - if any - is needed when making 
treatment and policy decisions. And it is very unusual for these other factors to be the same the world over" (Chalmers, 2010).

Ours is an era of chronic and expensive diseases. However, medical muddling is a profitable business, and the proliferation of new tests, devices, and drugs continues at an unprecedented pace. Although life would be simpler if these new technologies were always appraised in rigorous studies with clinically relevant endpoints, current data are often insufficient to guide practice (Naylor, 1995).

\section{Clinical Importance}

Using a common system for classification of caries lesion progression can facilitate communication between clinicians and visualize caries development characteristics for each patient. Following patient characteristics almost yearly enables clinicians to choose a caries risk assessment method tailored to their clinical question and setting, appropriate thresholds can then be applied.

Knowledge on evidence on caries risk assessment methods can further aid clinicians in their choice. Since previous caries experience express reasonable accuracy, and is established as an integral part of the clinical examination, the method could be an appropriate choice in caries risk assessment. Predicting health is more accurate than predicting disease, with this in mind the preventive care to healthy individuals can be reduced, which may be beneficial for both patients and clinics. 


\section{CONCLUSIONS}

- The results on diagnostic accuracy for the different methods of caries risk assessment analysed were insufficiently robust to determine the comparative accuracy of methods using microbiota vs. that of previous caries experience. The overall evidence for caries risk assessment methods previous caries experience and salivary mutans streptococci was low. Evidence on other analysed methods were lacking

- For the three clinically evaluated risk assessment methods, negative predictive values were high in general, indicating that it is more efficient to identify individuals at low risk of developing caries lesions

- Diagnostic test accuracy varies with threshold values of the index tests and of the reference standard. Furthermore, accuracy estimates vary with clinical variability

- Study design and reporting needs to be improved in caries risk assessment studies. The heterogeneity found in study design and in results of caries risk assessment studies suggest that a common approach on reference standard is desirable using bitewing radiography to assess caries lesion progression. This can improve validity and facilitate comparison of studies

- The classification system for the assessment of caries lesion progression in bitewing radiography demonstrated moderate to substantial rater agreement, although further studies of different samples and under different conditions are warranted 
- There is a need to develop novel approaches on caries risk assessment. A candidate biomarker, acid tolerance of dental biofilm, showed short-term stability, low intra-individual variation as well as substantial rater agreement. The method shows potential but further evaluation in prospective clinical trials are needed

- It is crucial to include experts from different fields when designing and performing a primary study, avoiding the pitfalls described in study I. Knowledge of diagnostic and predictive methods, research methodology as well as the actual condition/ disease researched is a prerequisite for successful planning and execution of studies. 


\section{FUTURE RESEARCH}

By now it is general knowledge that clinical methods for caries risk assessment display inherent deficiencies. At the time being, it may not be possible to quantify an objective probability of individual risk. There is ongoing research on the development of new clinical methods, such as the candidate method acid tolerance of dental biofilm. Candidate biomarkers need to be evaluated in prospective clinical trials and methods need to be chair-side in order to integrate to clinical everyday practice. Microbiological procedures used to predict caries must be scrutinised to the same extent as other diagnostic methods and their limitations must be set out clearly if the methodological quality of future research is to be improved.

We have established that characteristics of risk assessments methods alter depending on time, cofounding factors, disease spectrum, threshold values, validation methods for caries and caries progression. Therefore, in each clinic or region, with a defined patient sample, the aim of the risk assessment model needs to be established. This could include a local aim, such as making an effort to reduce the progression of enamel caries lesions, or a societal aim of reducing costs by prolonged visiting intervals for healthy individuals. Thus, the aim is dependent on the results one wishes to achieve. When the aim is settled, the appropriate risk assessment method can be chosen and implemented. After some time, the performance of the method in question needs to be evaluated systematically by the clinicians using it. It is only in their own patient sample, with their specific reliability and aim that they can accurately determine the efficacy. 
In the future, caries risk assessment methods probably have the ability to work, and maybe even to do it well. What if the main error in caries risk assessment is not the questionable accuracy, but rather that we do not understand risk as a concept in oral health care and research and thereby how to implement risk assessment methods to achieve the results we are hoping for? If there is a science of risk in dentistry, it is in its infancy, and we have far to go. 


\section{ACKNOWLEDGEMENTS}

This thesis would not have been possible without the valuable support and contribution from the people surrounding me. Thank you all. In particular, I wish to thank:

Madeleine Rohlin, for your commitment and for everything I have learnt. Your support has truly been exceptional. Your sharp eye for good research quality and novel ideas is something else. Thank you for giving me this opportunity, it has been a privilege.

Gunnel Svensäter, for your support and your creative big thinking. Thank you for sharing your outstanding expertise and, last but not least, for giving me the opportunity to continue doing research.

Nils-Eric Sahlin, for your important and difficult questions making us all think twice. Thank you for contributing with another perspective and for all the work with study I.

Jessica Neilands, my "go-to" person if in microbiological doubt. Thank you for walking me through the lab procedures and for all your work with the acid tolerance samples and manuscripts. Last but not least, thanks for nice chats.

Julia Davies, for critically revising texts and for your valuable support and contribution in studies III and IV. Also, thank you for all PhD student related questions you helped me with. 
Björn Axtelius, for giving another perspective in study IV and for keeping me updated through sending me interesting texts. Also, thank you for kindly answering my questions about statistics.

Christina Stebring-Franzon and Margareta Elfvin, for your commitment in the Borrow-project and for your contributions to study II.

Alvesta and Sölvesborg public dental clinics for the cooperation and efforts, making study II, III and IV possible. A special thanks to Maria Hilding at Alvesta clinic.

Ingegerd Mejàre, for contributing with your experience when working long and hard through study I.

Helena Christell, your smart and caring advice and ability to understand and listen means a lot. Thank you for helping me along, reading, commenting and with your esthetical eye revising my figures and soon my garden too. And above all, thank you for being my friend.

Christina Lindh, for generously sharing your knowledge and giving me opportunities, such as being a part of seminars and lectures. Thank you for encouraging me over the years.

Ayman Al-Okshi for nice cooperation with methodology seminars and friendly encouraging advice.

Mats Nilsson for assisting me with the design of the quality control of radiographic equipment.

My loving and supporting parents, sister and brothers, and all you other guys in my big and lovely family, can't cope without you!

Johan, my kind brother, for making the cover illustration of the thesis.

Linnea and Lovisa, my little stars. Love you more than you can ever imagine.

Erik, my calmer half. You made it possible. Thank you for looking out for my best at all times. Love you. 
This thesis was supported by the Borrow foundation, KOF-Syd (Clinical Research in Odontology, Sweden), the Swedish Dental Association, the Foundation for Dental Research in Malmö (for study III) and the Faculty of Odontology at Malmö University. 


\section{REFERENCES}

Alderson P. Absence of evidence is not evidence of absence. BMJ. 2004;328(7438):476-7.

Altman DG. Practical Statistics for Medical Research. 1st ed. London, UK: Chapman and Hall; 1991.

Altman DG, Bland JM. Absence of evidence is not evidence of absence. Aust Vet J. 1996 Oct;74(4):311.

Altman DG, Vergouwe Y, Royston P, Moons KG. Prognosis and prognostic research: validating a prognostic model. BMJ. 2009;338:b605. doi: 10.1136/ bmj.b605.

Amyes SGB. Kort om bakterier (In Swedish). Stockholm: Fri Tanke förlag; 2014.

Arnold WH, Gaengler P, Saeuberlich E. Distribution and volumetric assessment of initial approximal caries lesions in human premolars and permanent molars using computer-aided three-dimensional reconstruction. Arch Oral Biol. 2000;45(12):1065-71.

Atkins D, Best D, Briss PA, Eccles M, Falck-Ytter Y, Flottorp S, Guyatt GH, Harbour RT, Haugh MC, Henry D, Hill S, Jaeschke R, Leng G, Liberati A, Magrini N, Mason J, Middleton P, Mrukowicz J, O’Connell D, Oxman AD, Phillips B, Schünemann HJ, Edejer T, Varonen H, Vist GE, Williams JW Jr, Zaza S; GRADE Working Group. Grading quality of evidence and strength of recommendations. BMJ. 2004;328(7454):1490.

Axelsson S, Söder B, Nordenram G, Petersson LG, Dahlgren H, Norlund A, Källestål C, Mejàre I, Lingström P, Lagerlöf F, Holm AK, Twetman S. Effect of combined caries-preventive methods: a systematic review of controlled clinical trials. Acta Odontol Scand. 2004;62(3):163-9.

Baelum V, van Palenstein Helderman W, Hugoson A, Yee R, Fejerskov O. A global perspective on changes in the burden of caries and periodontitis: implications for dentistry. J Oral Rehabil. 2007;34(12):872-906. 
Bankel M, Robertson A, Köhler B. Carious lesions and caries risk predictors in a group of Swedish children 2 to 3 years of age. One year observation. Eur J Paediatr Dent. 2011;12(4):215-9.

Beck JD, Weintraub JA, Disney JA, Graves RC, Stamm JW, Kaste LM, Bohannan HM. University of North Carolina Caries Risk Assessment Study: comparisons of high risk prediction, any risk prediction, and any risk etiologic models. Community Dent Oral Epidemiol. 1992;20(6):313-21.

Beighton D. The complex oral microflora of high-risk individuals and groups and its role in the caries process. Community Dent Oral Epidemiol. 2005;33(4):248-55.

Bossuyt PM, Irwig L, Craig J, Glasziou P. Comparative accuracy: assessing new tests against existing diagnostic pathways. BMJ. 2006;332(7549):1089-92. Erratum in: BMJ. 2006 Jun 10;332(7554):1368.

Bossuyt PM, Reitsma JB, Bruns DE, Gatsonis CA, Glasziou PP, Irwig L, Lijmer JG, Moher D, Rennie D, de Vet HC, Kressel HY, Rifai N, Golub RM, Altman DG, Hooft L, Korevaar DA, Cohen JF; STARD Group. STARD 2015: An updated list of essential items for reporting diagnostic accuracy studies. Clin Chem. 2015;61:1446-52. doi: 10.1373/clinchem.2015.246280.

Bossuyt PM, Reitsma JB, Bruns DE, Gatsonis CA, Glasziou PP, Irwig LM, Lijmer JG, Moher D, Rennie D, De Vet HC: Standards for reporting of diagnostic accuracy: Towards complete and accurate reporting of studies of diagnostic accuracy: the STARD initiative. Standards for Reporting of Diagnostic Accuracy. Clin Chem. 2003;49:1-6.

Bratthall D, Hänsel Petersson G. Cariogram -a multifactorial risk assessment model for a multifactorial disease. Community Dent Oral Epidemiol. 2005;33(4):256-64.

Brinck I, Halldén S, Maurin A-S, Persson J (eds). Risk and the vital human. Nora: Nya Doxa; 2005.

Brown P, Brunnhuber K, Chalkidou K, I Chalmers, M Clarke, M Fenton, C Forbes, J Glanville, N J Hicks, J Moody, S Twaddle, H Timimi, P Young. How to formulate research recommendations. BMJ. 2006;333:804-6.

Campus G, Cagetti MG, Sale S, Carta G, Lingström P. Cariogram validity in schoolchildren: a two-year follow-up study. Caries Res. 2012;46(1):16-22. doi: $10.1159 / 000334932$.

Centre for Reviews and Dissemination. Systematic reviews: CRD's guidance for undertaking reviews in health care. York: York Publishing Services Ltd.; 2009 (cited April 30th, 2017). Available from: http://www.york.ac.uk/inst/ crd/pdf/Systematic_Reviews.pdf.

Chalmers I. Systematic reviews and uncertainties about the effects of treatments [editorial]. Cochrane Database of Systematic Reviews 2010;(5):10.1002/14651858.ED000004. 
Chien PF, Khan KS. Evaluation of a clinical test. II: Assessment of validity. BJOG. 2001;108(6):568-72.

Christensen LB, Twetman S, Sundby A. Oral health in children and adolescents with different socio-cultural and socio-economic backgrounds. Acta Odontol Scand. 2010;68(1):34-42. doi: 10.3109/00016350903301712.

Cohen JF, Korevaar DA, Altman DG, Bruns DE, Gatsonis CA, Hooft L, Irwig L, Levine D, Reitsma JB, de Vet HC, Bossuyt PM. STARD 2015 guidelines for reporting diagnostic accuracy studies: explanation and elaboration. BMJ Open. 2016;6(11):e012799. doi: 10.1136/bmjopen-2016-012799.

Collins GS, Reitsma JB, Altman DG, Moons KG. Transparent Reporting of a multivariable prediction model for Individual Prognosis or Diagnosis (TRIPOD): the TRIPOD statement. Ann Intern Med. 2015;162(1):55-63. doi: 10.7326/M14-0697.

Coogan MM, Mackeown JM, Galpin JS, Fatti LP. Microbiological impressions of teeth, saliva and dietary fibre can predict caries activity. J Dent. 2008;36(11):892-9. doi: 10.1016/j.jdent.2008.07.004.

Corbett ME, Moore WJ. Distribution of dental caries in ancient British populations. IV. The 19th century. Caries Res. 1976;10(6):401-14.

David J, Raadal M, Wang NJ, Strand GV. Caries increment and prediction from 12 to 18 years of age: a follow-up study. Eur Arch Paediatr Dent. 2006;7(1):31-7.

Deeks JJ. Systematic reviews in health care: Systematic reviews of evaluations of diagnostic and screening tests. BMJ. 2001;323(7305):157-62.

Demers M, Brodeur JM, Mouton C, Simard PL, Trahan L, Veilleux G. A multivariate model to predict caries increment in Montreal children aged 5 years. Community Dent Health. 1992;9(3):273-81.

Disney JA, Graves RC, Stamm JW, Bohannan HM, Abernathy JR, Zack DD. The University of North Carolina Caries Risk Assessment study: further developments in caries risk prediction. Community Dent Oral Epidemiol. 1992;20(2):64-75.

Do LG, Spencer A. Oral health-related quality of life of children by dental caries and fluorosis experience. J Public Health Dent. 2007;67(3):132-9.

Dukic W, Delija B, Lulic Dukic O. Caries prevalence among schoolchildren in Zagreb, Croatia. Croat Med J. 2011;52(6):665-71.

Ekstrand KR, Ricketts DN, Kidd EA. Occlusal caries: pathology, diagnosis and logical management. Dent Update 2001;28:380-7.

Espelid I, Tveit AB, Mejàre I, Sundberg H, Hallonsten AL. Restorative treatment decisions on occlusal caries in Scandinavia. Acta Odontol Scand. 2001;59(1):21-7. 
Espelid I, Tveit AB, Riordan PJ. Radiographic caries diagnosis by clinicians in Norway and Western Australia. Community Dent Oral Epidemiol. 1994;22(4):214-9.

Equator center, the Centre for Statistics in Medicine. The Equator Network. Oxford, UK: University of Oxford (cited April 30th, 2017). Available at: www.equator-network.org.

Feinstein AR. Misguided efforts and future challenges for research on "diagnostic tests”. J Epidemiol Comm Health. 2002;56:330e2.

Fejerskov O, Guldager Bilde P, Bizzarro M, Connelly JN, Skovhus Thomsen J, Nyvad B. Dental caries in Rome, 50-100 AD. Caries Res. 2012;46(5):46773. doi: 10.1159/000339664.

Filstrup SL, Briskie D, da Fonseca M, Lawrence L, Wandera A, Inglehart MR. Early childhood caries and quality of life: child and parent perspectives. Pediatr Dent. 2003;25(5):431-440.

Fonseca AA, Cherubini K, Veeck EB, Ladeira RS, Carapeto LP. Effect of 10\% formalin on radiographic optical density of bone specimens. Dentomaxillofac Radiol. 2008;37(3):137-41. doi: 10.1259/dmfr/18109064.

Fryback DG, Thornbury JR. The efficacy of diagnostic imaging. Med Decis Making. 1991 Apr;11(2):88-94.

Gao SS, Zhang S, Mei ML, Lo EC, Chu CH. Caries remineralisation and arresting effect in children by professionally applied fluoride treatment - a systematic review. BMC Oral Health. 2016;16:12. doi: 10.1186/s12903016-0171-6.

Gimenez T, Piovesan C, Braga MM, Raggio DP, Deery C, Ricketts DN, Ekstrand KR, Mendes FM. Visual Inspection for Caries Detection: A Systematic Review and Meta-analysis. J Dent Res. 2015;94(7):895-904. doi: 10.1177/0022034515586763.

Gordan VV, Garvan CW, Heft MW, Fellows JL, Qvist V, Rindal DB, Gilbert GH; DPBRN Collaborative Group. Restorative treatment thresholds for interproximal primary caries based on radiographic images: findings from the Dental Practice-Based Research Network. Gen Dent. 2009;57(6):654-63.

Grimes DA, Schulz KF. Refining clinical diagnosis with likelihood ratios. Lancet. 2005;29;365(9469):1500-5.

Griner PF, Mayewski RJ, Mushlin AI, Greenland P. Selection and interpretation of diagnostic tests and procedures. Principles and applications. Ann Intern Med. 1981;94(4 Pt 2):557-92.

Grindefjord M, Dahllöf G, Modéer T. Caries development in children from 2.5 to 3.5 years of age: a longitudinal study. Caries Res. 1995; 29:449-54.

Gröndahl H-G. Radiologic diagnosis in caries management. In: Thylstrup A, Fejerskov O, eds. Textbook of clinical cariology. 2nd ed. Copenhagen: Munksgaard; 1994. 
Gröndahl HG. Radiographic caries diagnosis. A study of caries progression and observer performance. Swed Dent J Suppl. 1979;(3):1-32.

Gröndahl H-G, Hollender L, Malmcrona E, Sundquist B. Dental caries and restorations in teenagers. I. Index and score system for radiographic studies of proximal surfaces. Swed Dent J. 1977;1:45-50.

Gualandi PB. Food habits and dental disease in an iron-age population. Anthropol Anz. 1992;50(1-2):67-82.

Hausen H. Caries prediction -state of the art. Community Dent Oral Epidemiol. 1997;25(1):87-96.

Hausen H, Kärkkäinen S, Seppä L. Application of the high-risk strategy to control dental caries. Community Dent Oral Epidemiol. 2000;28(1):26-34.

Helderman WH, Mulder J, van T Hof MA, Truin GJ. Validation of a Swiss method of caries prediction in Dutch children. Community Dent Oral Epidemiol. 2001;29(5):341-5.

Hintze H, Wenzel A. Diagnostic outcome of methods frequently used for caries validation. A comparison of clinical examination, radiography and histology following hemisectioning and Serial Tooth Sectioning. Caries Res. 2003;37:115-24. DOI: 10.1159/000069016.

Hintze H, Wenzel A. Influence of the validation method on diagnostic accuracy for caries. A comparison of six digital and two conventional radiographic systems. Dentomaxillofac Radiol. 2002;31(1):44-9.

Hintze H, Wenzel A, Larsen MJ. Stereomicroscopy, film radiography, microradiography and naked-eye inspection of tooth sections as validation for occlusal caries diagnosis. Caries Res. 1995;29(5):359-63.

Hlatky MA, Pryor DB, Harrell FE Jr, Califf RM, Mark DB, Rosati RA. Factors affecting sensitivity and specificity of exercise electrocardiography. Multivariable analysis. Am J Med. 1984;77(1):64-71.

Honest H, Khan KS. Reporting of measures of accuracy in systematic reviews of diagnostic literature. BMC Health Serv Res. 2002;2:4.

Hänsel Petersson G, Twetman S, Bratthall D. Evaluation of a computer program for caries risk assessment in schoolchildren. Caries Res. 2002;36(5):327-40.

Ioannidis JP, Greenland S, Hlatky MA, Khoury MJ, Macleod MR, Moher $\mathrm{D}$, Schulz KF, Tibshirani R. Increasing value and reducing waste in research design, conduct, and analysis. Lancet. 2014;383(9912):166-75. doi: 10.1016/S0140-6736(13)62227-8.

Irwig L, Macaskill P, Glasziou P, Fahey M. Meta-analytic methods for diagnostic test accuracy. J Clin Epidemiol. 1995;48(1):119-30.

Ismail A. Diagnostic levels in dental public health planning. Caries Res. 2004;38(3):199-203. 
Ismail AI, Lim S, Sohn W. A transition scoring system of caries increment with adjustment of reversals in longitudinal study: evaluation using primary tooth surface data. Community Dent Oral Epidemiol. 2011;39(1):61-8. doi: 10.1111/j.1600-0528.2010.00565.x.

Ismail AI, Sohn W, Tellez M, Willem JM, Betz J, Lepkowski J. Risk indicators for dental caries using the International Caries Detection and Assessment System (ICDAS). Community Dent Oral Epidemiol. 2008;36(1):55-68. doi: 10.1111/j.1600-0528.2006.00369.x.

Ismail AI, Sohn W, Tellez M, Amaya A, Sen A, Hasson H, Pitts NB. The International Caries Detection and Assessment System (ICDAS): an integrated system for measuring dental caries. Community Dent Oral Epidemiol. 2007;35(3):170-8.

Ivanković A, Lukić IK, Ivanković Z, Radić A, Vukić I, Simić A. Dental caries in postwar Bosnia and Herzegovina. Community Dent Oral Epidemiol. 2003;31(2):100-4.

Ivers NM, Grimshaw JM. Reducing research waste with implementation laboratories. Lancet. 2016;388(10044):547-8. doi: 10.1016/S01406736(16)31256-9.

Jenkinson HF. Beyond the oral microbiome. Environ Microbiol. 2011;13(12):3077-3087.

Keene HJ, Shklair IL. Relationship of Streptococcus mutans carrier status to the development of carious lesions in initially cariesfree recruits. J Dent Res. 1974 Sep-Oct;53(5):1295.

Keynes. A Treatise on Probability. Scholar Choice. Rochester; Macmillan \& Co.; 2015.

Kidd EA, Naylor MN, Wilson RF. Prevalence of clinically undetected and untreated molar occlusal dentine caries in adolescents on the Isle of Wight. Caries Res. 1992;26(5):397-401.

Kim JS, Kim DK, Hong SJ. Assessment of errors and misused statistics in dental research. Int Dent J. 2011;61(3):163-7. doi: 10.1111/j.1875595X.2011.00037.

Kingman A, Little W, Gomez I, Heifetz SB, Driscoll WS, Sheats R, Supan P. Salivary levels of Streptococcus mutans and lactobacilli and dental caries experiences in a US adolescent population. Community Dent Oral Epidemiol. 1988;16(2):98-103.

Korevaar DA, Wang J, van Enst WA, Leeflang MM, Hooft L, Smidt N, et al. Reporting diagnostic accuracy studies: some improvements after 10 years of STARD. Radiology. 2015;274:781-9.

Kottner J, Audige L, Brorson S, Donner A, Gajewski BJ, Hróbjartsson A, Roberts C, Shoukri M, Streiner DL. Guidelines for Reporting Reliability and Agreement Studies (GRRAS) were proposed. Int J Nurs Stud. 2011a;48(6):661-71. doi: 10.1016/j.ijnurstu.2011.01.016. 
Kottner J, Dassen T, Tannen A. Inter- and intrarater reliability of the Waterlow pressure sore risk scale: a systematic review. Int J Nurs Stud. 2009;46:369e79.

Kottner J, Dassen T. Interpreting interrater reliability coefficients of the Braden scale: A discussion paper. Int J Nurs Stud. 2008;45:1239e46.

Kottner J, Streiner DL. The difference between reliability and agreement. J Clin Epidemiol. 2011b;64(6):701-2; author reply 702. doi: 10.1016/j. jclinepi.2010.12.001.

Kullendorff B, Gröndahl K, Rohlin M, Henrikson CO. Subtraction radiography for the diagnosis of periapical bone lesions. Endod Dent Traumatol. 1988;4(6):253-9.

Künzel W. Trends in caries experience of 12-year-old children in east European countries. Int J Paediatr Dent. 1996;6(4):221-6.

Lachs MS, Nachamkin I, Edelstein PH, Goldman J, Feinstein AR, Schwartz JS. Spectrum bias in the evaluation of diagnostic tests: lessons from the rapid dipstick test for urinary tract infection. Ann Intern Med. 1992;117:135-40.

Lane N. The unseen world: reflections on Leeuwenhoek (1677) 'Concerning little animals’. Philos Trans R Soc Lond B Biol Sci. 2015;370(1666). doi: 10.1098/rstb.2014.0344.

Landis JR, Koch GG. The measurement of observer agreement for categorical data. Biometrics 1977;33:159-74. doi: 10.2307/2529310.

Leeflang MM1, Bossuyt PM, Irwig L. Diagnostic test accuracy may vary with prevalence: implications for evidence-based diagnosis. J Clin Epidemiol. 2009;62(1):5-12. doi: 10.1016/j.jclinepi.2008.04.007. Epub 2008 Sep 7.

Leeflang MM, Deeks JJ, Gatsonis C, Bossuyt PM; Cochrane Diagnostic Test Accuracy Working Group. Systematic reviews of diagnostic test accuracy. Ann Intern Med. 2008;149(12):889-97.

Leeflang MM, Rutjes AW, Reitsma JB, Hooft L, Bossuyt PM. Variation of a test's sensitivity and specificity with disease prevalence. CMAJ. 2013;185(11):E537-44. doi: 10.1503/cmaj.121286.

van Leeuwenhoek A. An abstract of a letter from Antonie van Leeuwenhoek, about animals in the scurf of the teeth. Philos Trans R Soc Lond. 1684;14:568-74.

Li Y, Wang W. Predicting caries in permanent teeth from caries in primary teeth: an eight-year cohort study. J Dent Res. 2002;81(8):561-6.

Lijmer JG, Mol BW, Heisterkamp S, Bonsel GJ, Prins MH, van der Meulen JH, Bossuyt PM. Empirical evidence of design-related bias in studies of diagnostic tests. JAMA. 1999;282(11):1061-6. Erratum in: JAMA 2000;283(15):1963. 
Lingström P, van Ruyven FO, van Houte J, Kent R. The pH of dental plaque in its relation to early enamel caries and dental plaque flora in humans. J Dent Res. 2000;79(2):770-7.

Lith A, Lindstrand C, Gröndahl HG. Caries development in a young population managed by a restrictive attitude to radiography and operative intervention: II. A study at the surface level. Dentomaxillofac Radiol. 2002;31(4):232-9.

Loesche WJ. Role of Streptococcus mutans in human dental decay. Microbiol Rev. 1986;50(4):353-80.

Loesche WJ, Straffon LH. Longitudinal investigation of the role of Streptococcus mutans in human fissure decay. Infect Immun. 1979;26(2):498-507.

Longbottom C, Ekstrand K, Zero D, Kambara M. Novel preventive treatment options. Monogr Oral Sci. 2009;21:156-63. doi: 10.1159/000224220.

Loong TW. Understanding sensitivity and specificity with the right side of the brain. BMJ. 2003;327(7417):716-9.

Luan W, Baelum V, Fejerskov O, Chen X. Ten-year incidence of dental caries in adult and elderly Chinese. Caries Res. 2000;34(3):205-13.

Lukacs JR. Dental paleopathology and agricultural intensification in South Asia: new evidence from Bronze Age Harappa. Am J Phys Anthropol. 1992;87:133-50.

Macaskill P, Gatsonis C, Deeks JJ, Harbord RM, Takwoingi Y. Chapter 10: Analysing and Presenting Results. In: Deeks JJ, Bossuyt PM, Gatsonis C (editors), Cochrane Handbook for Systematic Reviews of Diagnostic Test Accuracy Version 1.0. The Cochrane Collaboration, 2010 (citer April 30, 2017). Available from: http://srdta.cochrane.org/.

Manji F, Fejerskov O, Baelum V. Pattern of dental caries in an adult rural population. Caries Res. 1989;23(1):55-62.

Manzi G, Salvadei L, Vienna A, Passarello P. Discontinuity of life conditions at the transition from the Roman imperial age to the early middle ages: Example from central Italy evaluated by pathological dento-alveolar lesions. Am J Hum Biol. 1999;11(3):327-41.

Marsh PD. Are dental diseases examples of ecological catastrophes? Microbiology 2003;149:279-94.

Marsh PD. Microbial ecology of dental plaque and its significance in health and disease. Adv Dent Res. 1994;8:263-71.

Marsh PD. The significance of maintaining the stability of the natural microflora of the mouth. Br Dent J. 1991;171(6):174-7.

Marsh PD, Bradshaw DJ. Physiological approaches to the control of oral biofilms. Adv Dent Res. 1997;11(1):176-85. 
Marsh PD, Featherstone A, McKee AS, Hallsworth AS, Robinson C, Weatherell JA, Newman HN, Pitter AF. A microbiological study of early caries of approximal surfaces in schoolchildren. J Dent Res. 1989;68(7):1151-4.

Mattos-Graner RO, Smith DJ, King WF, Mayer MP. Water-insoluble glucan synthesis by mutans streptococcal strains correlates with caries incidence in 12- to 30-month-old children. J Dent Res. 2000;79(6):1371-7.

Mejàre I, Axelsson S, Dahlén G, Espelid I, Norlund A, Tranæus S, Twetman S. Caries risk assessment. A systematic review. Acta Odontol Scand. 2014;72(2):81-91. doi: 10.3109/00016357.2013.822548.

Mejàre IA, Klingberg G, Mowafi FK, Stecksén-Blicks C, Twetman SHA, Tranæus SH. A systematic map of systematic reviews in pediatric dentistry—what do we really know? PlosOne 2015;10:1-21.

Mejàre I, Stenlund H, Zelezny-Holmlund C. Caries incidence and lesion progression from adolescence to young adulthood: a prospective 15 -year cohort study in Sweden. Caries Res. 2004;38(2):130-41.

Meurman PK, Pienihäkkinen K. Factors associated with caries increment: a longitudinal study from 18 months to 5 years of age. Caries Res. 2010;44(6):519-24. doi: 10.1159/000320717.

Miller WD. The Micro-organisms of the Human Mouth. The Local and General Diseases which are Caused by them. Philadelphia: The S.S. White Dental Mfg. Co.; 1890.

Moher D, Liberati A, Tetzlaff J, Altman DG; PRISMA Group. Preferred reporting items for systematic reviews and meta-analyses: the PRISMA statement. PLoS Med. 2009;6(7):e1000097. doi: 10.1371/journal. pmed.1000097.

Moons KG, Kengne AP, Woodward M, Royston P, Vergouwe Y, Altman DG, Grobbee DE. Risk prediction models: I. Development, internal validation, and assessing the incremental value of a new (bio)marker. Heart. 2012;98(9):683-90. doi: 10.1136/heartjnl-2011-301246.

Moore WJ, Corbett ME. Distribution of dental caries in ancient British populations. III. The 17th century. Caries Res. 1975;9(2):163-75.

Mulherin SA, Miller WC. Spectrum bias or spectrum effect? Subgroup variation in diagnostic test evaluation. Ann Intern Med. 2002;137:598e602.

Naylor CD. Grey zones of clinical practice: some limits to evidence-based medicine. Lancet. 1995;345(8953):840-2.

Neilands J, Petersson LG, Beighton D, Svensäter G. Fluoride-supplemented milk inhibits acid tolerance in root caries biofilms. Caries Res. 2012;46(2):156-60. doi: 10.1159/000337390.

Patel R. N, Eaton, K. A, Pitts N, Schulte A, Pieper K, White S. Variation in methods used to determine national mean DMFT scores for 12-year-old children in European countries. Community Dent Health. 2016;4(33):28691. 
Penelope research. London, UK (cited March 14th, 2017). Available from: www.peneloperesearch.com.

Petersen, PE. Oral Health. International Encyclopedia of Public Health. 2008;4:677-85. (Available from: https://www.mah.se/upload/FAKULTETER/OD/Avdelningar/who/doc/EncycPubORHpep.pdf.

Petersen PE. Challenges to improvement of oral health in the 21st century -the approach of the WHO Global Oral Health Programme. Int Dent J. 2004;54(6 Suppl 1):329-43.

Petersen PE, Ogawa H. Prevention of dental caries through the use of fluoride -the WHO approach. Community Dent Health. 2016;33(2):66-8.

Petersson GH, Isberg PE, Twetman S. Caries risk assessment in school children using a reduced Cariogram model without saliva tests. BMC Oral Health. 2010;10:5. doi: 10.1186/1472-6831-10-5.

Petti S, Hausen HW. Caries prediction by multiple salivary mutans streptococcal counts in caries-free children with different levels of fluoride exposure, oral hygiene and sucrose intake. Caries Res. 2000;34:380-7.

Pienihäkkinen K, Jokela J, Alanen P. Assessment of caries risk in preschool children. Caries Res. 2004;38:156-62.

Pitts NB. Diagnostic methods for caries: what is appropriate when? J Dent. 1991 Dec;19(6):377-82.

Pitts NB, Fyffe HE. The effect of varying diagnostic thresholds upon clinical caries data for a low prevalence group. J Dent Res. 1988;67(3):592-6.

Pitts NB, Wefel JS. Remineralization/desensitization: what is known? What is the future? Adv Dent Res. 2009;21(1):83-6. doi: $10.1177 / 0895937409335644$.

Pliskin JS, Shwartz M, Gröndahl HG, Boffa J. Reliability of coding depth of approximal carious lesions from non-independent interpretation of serial bitewing radiographs. Community Dent Oral Epidemiol. 1984;12:366-70. doi: http://dx.doi.org/10.1111/j.1600-0528.1984.tb01473.x.

Ratanawongsa N, Thomas PA, Marinopoulos SS, Dorman T, Wilson LM, Ashar BH, Magaziner JL, Miller RG, Prokopowicz GP, Qayyum R, Bass EB. The reported validity and reliability of methods for evaluating continuing medical education: a systematic review. Acad Med. 2008;83(3):274-83. doi: 10.1097/ACM.0b013e3181637925.

Ridell K, Olsson H, Mejàre I. Unrestored dentin caries and deep dentin restorations in Swedish adolescents. Caries Res. 2008;42(3):164-70. doi: 10.1159/000128560.

Rifai N, Gillette MA, Carr SA. Protein biomarker discovery and validation: the long and uncertain path to clinical utility. Nat Biotechnol. 2006;24(8):971-83. 
Rimmer PA, Pitts NB. Effects of diagnostic threshold and overlapped approximal surfaces on reported caries status. Community Dent Oral Epidemiol. 1991;19(4):205-12.

Robinson PJ. Radiology's Achilles' heel: error and variation in the interpretation of the Röntgen image. Br J Radiol. 1997;70:1085-98.

Royston P, Altman DG. Visualizing and assessing discrimination in the logistic regression model. Stat Med. 2010 Oct 30;29(24):2508-20. doi: 10.1002/ sim.3994.

Rutjes AW, Reitsma JB, Di Nisio M, Smidt N, van Rijn JC, Bossuyt PM. Evidence of bias and variation in diagnostic accuracy studies. CMAJ. 2006;174(4):469-76.

Sánchez-Pérez L, Golubov J, Irigoyen-Camacho ME, Moctezuma PA, AcostaGio E. Clinical, salivary, and bacterial markers for caries risk assessment in schoolchildren: a 4-year follow-up. Int J Paediatr Dent. 2009;19(3):186-92.

SBU. Att förebygga karies. En systematisk litteraturöversikt (In Swedish). Stockholm: The Swedish Council on Technology Assessment in Health Care (SBU); 2002. SBU report nr 161. ISBN 91-87890-81-X. English summary available at: http://www.sbu.se/globalassets/publikationer/content0/1/dentalcare_2002/dentalcarieslut.pdf (cited April 29, 2017).

SBU. Karies - diagnostik, riskbedömning och icke-invasiv behandling. En systematisk litteraturöversikt (In Swedish). Stockholm: The Swedish Council on Technology Assessment in Health Care (SBU); 2007. SBU report nr 188. ISBN: 978-91-85413-21-8. English summary available at: http://www.sbu. se/en/publications/sbu-assesses/caries--diagnosis-risk-assessment-and-noninvasive-treatment/ (cited April 29, 2017).

SBU. Kronisk parodontit - prevention, diagnostik och behandling. En systematisk litteraturöversikt (In Swedish). Stockholm: The Swedish Council on Technology Assessment in Health Care (SBU); 2004. SBU report nr 169. ISBN 91-87890-96-8. English summary available at: http:/www.sbu.se/ globalassets/publikationer/content1/1/sbu_parodontit_eng.pdf (cited April 30, 2017)

Schwendicke F, Tzschoppe M, Paris S. Radiographic caries detection: A systematic review and meta-analysis. J Dent. 2015;43(8):924-33. doi: 10.1016/j. jdent.2015.02.009.

Selwitz RH, Ismail AI, Pitts NB. Dental caries. Lancet 2007;369:53-9.

Skeie MS, Raadal M, Strand GV, Espelid I. The relationship between caries in the primary dentition at 5 years of age and permanent dentition at 10 years of age - a longitudinal study. Int J Paediatr Dent. 2006;16(3):152-60.

Senel B, Kamburoglu K, Uçok O, Y“uksel SP, Ozen T, Avsever H. Diagnostic accuracy of different imaging modalities in detection of proximal caries. Dentomaxillofac Radiol. 2010;39:501-11. doi: http://dx.doi.org/10.1259/ $\mathrm{dmfr} / 28628723$. 
Shea BJ, Grimshaw JM, Wells GA, Boers M, Andersson N, Hamel C, Porter AC, Tugwell P, Moher D, Bouter LM. Development of AMSTAR: a measurement tool to assess the methodological quality of systematic reviews. BMC Med Res Methodol. 2007;7:10.

Simón-Soro A, Mira A. Solving the etiology of dental caries. Trends Microbiol. 2015;23(2):76-82. doi: 10.1016/j.tim.2014.10.010.

de Soet JJ, Nyvad B, Kilian M. Strain-related acid production by oral streptococci. Caries Res. 2000;34:486-90.

Stern RH. Individual risk. J Clin Hypertens. 2012;14(4):261-4. doi: 10.1111/j.1751-7176.2012.00592.x.

Stochkendahl MJ, Christensen HW, Hartvigsen J, Vach W, Haas M, Hestbaek L, Adams A, Bronfort G. Manual examination of the spine: a systematic critical literature review of reproducibility. J Manipulative Physiol Ther. 2006;29(6):475-85.

Streiner DL, Norman GR. Health measurement scales. 4th edition. Oxford, UK: Oxford University Press; 2008.

Streiner DL. Breaking up is hard to do: the heartbreak of dichotomizing continuous data. Can J Psychiatry. 2002;47(3):262-6.

Sullivan A, Schröder U. Systematic analysis of gingival state and salivary variables as predictors of caries from 5 to 7 years of age. Scand J Dent Res. 1989;97(1):25-32.

Swets JA, Pickett RM. Evaluation of diagnostic systems: methods from signal detection theory. New York, USA: Academic Press; 1982.

Tanzer JM, Livingston J, Thompson AM. The microbiology of primary dental caries in humans. J Dent Educ. 2001;65(10):1028-37.

Tellez M, Gomez J, Pretty I, Ellwood R, Ismail AI. Evidence on existing caries risk assessment systems: are they predictive of future caries? Community Dent Oral Epidemiol. 2013a;41(1):67-78. doi: 10.1111/cdoe.12003.

Tellez M, Gomez J, Kaur S, Pretty IA, Ellwood R, Ismail AI. Non-surgical management methods of noncavitated carious lesions. Community Dent Oral Epidemiol. 2013b;41(1):79-96. doi: 10.1111/cdoe.12028.

The Swedish National Board of Health and Welfare (Socialstyrelsen). Caries in children and adolescents, epidemiologic information from 2015 (In Swedish). Sweden: The Swedish National Board of Health and Welfare; 2017 (cited April 30th, 2017). Available at: http://www.socialstyrelsen.se/ publikationer2015/2015-3-20.

The Swedish National Board of Health and Welfare (Socialstyrelsen). Caries in children and adolescents, epidemiologic information from 2013 (In Swedish). Sweden: The Swedish National Board of Health and Welfare; 2013, updated 2015. (cited April 30th, 2017). Available at: http://www.socialstyrelsen.se/publikationer2015/2015-3-20. 
Topić B, Raščić-Konjhodžić H, Cižek Sajko M. Periodontal disease and dental caries from Krapina Neanderthal to contemporary man -skeletal studies. Acta Med Acad. 2012;41(2):119-30. doi: 10.5644/ama2006-124.45.

Twetman S. Caries risk assessment in children: how accurate are we? Eur Arch Paediatr Dent. 2016;17(1):27-32. doi: 10.1007/s40368-015-0195-7.

U.S. National Library of Medicine, National Center for Biotechnology Information. PubMed database MeSH "Diagnostic Tests Routine”. Bethesda: U.S. National Library of Medicine. (cited April 30th, 2017a). Available from: https://www.ncbi.nlm.nih.gov/mesh/68003955.

U.S. National Library of Medicine, National Center for Biotechnology Information. PubMed database MeSH "Risk". Bethesda: U.S. National Library of Medicine. (cited April 30th, 2017b). Available from: gov.proxy.mah.se/ $\mathrm{mesh} /$ ?term=relative+risk.

de Vet HC, Mokkink LB, Terwee CB, Hoekstra OS, Knol DL. Clinicians are right not to like Cohens $\kappa$. BMJ 2013;346:f2125. doi: 10.1136/bmj.f2125.

de Vet HC, Terwee CB, Knol DL, Bouter LM. When to use agreement versus reliability measures. J Clin Epidemiol. 2006;59(10):1033-9.

Vidnes-Kopperud S, Tveit AB, Espelid I. Changes in the treatment concept for approximal caries from 1983 to 2009 in Norway. Caries Res. 2011;45(2):113-20. doi: 10.1159/000324810.

Walter SD, Macaskill P, Lord SJ, Irwig L. Effect of dependent errors in the assessment of diagnostic or screening test accuracy when the reference standard is imperfect. Stat Med. 2012 May 20;31(11-12):1129-38. doi: 10.1002/sim.4444.

Weinstein MC, Fineberg HV, Elstein AS., Frazier HS, Neuhauser D, Neutra RR, McNeil BJ. Clinical decision analysis. Philadelphia; Saunders; 1980.

Welin-Neilands J, Svensäter G. Acid tolerance of biofilm cells of Streptococcus mutans. Appl Environ Microbiol. 2007;73:5633-38.

Wenzel A. Radiographic display of carious lesions and cavitation in approximal surfaces: Advantages and drawbacks of conventional and advanced modalities. Acta Odontol Scand. 2014;72(4):251-64. doi: 10.3109/00016357.2014.888757.

Wenzel A. Computer-automated caries detection in digital bitewings: consistency of a program and its influence on observer agreement. Caries Res. 2001;35:12-20. doi: http://dx.doi.org/10.1159/000047425.

Wenzel A. Digital radiography and caries diagnosis. Dentomaxillofac Radiol. 1998;27: 3-11. doi: http://dx.doi.org/10.1038/sj.dmfr.4600321.

Wenzel A, Anthonisen PN, Juul MB. Reproducibility in the assessment of caries lesion behavior: a comparison between conventional film and subtraction radiography. Caries Res. 2000;34:214-8. doi: http://dx.doi. org/10.1159/000016593. 
Wenzel A, Hintze H. Comparison of microscopy and radiography as gold standards in radiographic caries diagnosis. Dentomaxillofac Radiol. 1999a;28(3):182-5.

Wenzel A, Hintze $\mathrm{H}$. The choice of gold standard for evaluating tests for caries diagnosis. Dentomaxillofac Radiol. 1999b;28(3):132-6.

Wenzel A, Hintze H. Perception of image quality in direct digital radiography after application of various image treatment filters for detectability of dental disease. Dentomaxillofac Radiol. 1993;22(3):131-4.

Wenzel A, Larsen MJ, Fejerskov O. Detection of occlusal caries without cavitation by visual inspection, film radiographs, xeroradiographs, and digitized radiographs. Caries Res. 1991;25(5):365-71.

Wenzel A, Pitts N, Verdonschot EH, Kalsbeek H. Developments in radiographic caries diagnosis. J Dent. 1993;21(3):131-40.

Whiting PF, Rutjes AW, Westwood ME, Mallett S; QUADAS-2 Steering Group. A systematic review classifies sources of bias and variation in diagnostic test accuracy studies. J Clin Epidemiol. 2013;66(10):1093-104. doi: 10.1016/j.jclinepi.2013.05.014.

Whiting PF, Rutjes AW, Westwood ME, Mallett S, Deeks JJ, Reitsma JB, et al. QUADAS-2: a revised tool for the quality assessment of diagnostic accuracy studies. Ann Intern Med. 2011;155(8):529-536.

Whiting P, Harbord R, de Salis I, Egger M, Sterne J. Evidence-based diagnosis. J Health Serv Res Policy. 2008;13 Suppl 3:57-63. doi: 10.1258/ jhsrp.2008.008025.

Whiting P, Harbord R, Kleijnen J. No role for quality scores in systematic reviews of diagnostic accuracy studies. BMC Med Res Methodol. 2005;5:19. doi: 10.1186/1471-2288-5-19.

Whiting P, Rutjes AW, Reitsma JB, Glas AS, Bossuyt PM, Kleijnen J. Sources of variation and bias in studies of diagnostic accuracy: a systematic review. Ann Intern Med. 2004;140(3):189-202.

Whiting P, Rutjes AWS, Reitsma JB, Bossuyt PMM, Jos Kleijnen. The development of QUADAS: a tool for the quality assessment of studies of diagnostic accuracy included in systematic reviews. BMC Med Res Methodol. 2003;3:25. doi: 10.1186/1471-2288-3-25.

WHO, World Health Organization. Oral health information systems. Oral health surveillance. 2017 (cited April 30, 2017) Available at: http://www. who.int/oral_health/action/information/surveillance/en/.

WHO, World Health Organization, report. Oral health: action plan for promotion and integrated disease prevention. Executive board 120th session, provisional agenda item 4.6. EB120/10, 2007 (cited April 30, 2017). Available from: http://apps.who.int/iris/handle/10665/21909.

WHO, World Health Organization. Health Surveys. Basic Methods. 4th edn. Geneva: World Health Organization. 1997. 
WHO, World Health Organization. Oral health database. Malmö, Sweden: Malmö university (cited April 30th, 2017). Available from: https://www. mah.se/CAPP/Methods-and-Indices/for-Caries-prevalence/.

Yerushalmy J. Statistical problems in assessing methods of medical diagnosis, with special reference to X-ray techniques. Public Health Rep. 1947;62(40):1432-49.

Zamora J, Abraira V, Muriel A, Khan K, Coomarasamy A. Meta-DiSc: a software for meta-analysis of test accuracy data. BMC Med Res Methodol. 2006;6:31.

Zero D, Fontana M, Lennon AM. Clinical applications and outcomes of using indicators of risk in caries management. J Dent Educ. 2001;65(10):112632.

Zhang Q, van Palenstein Helderman WH. Caries experience variables as indicators in caries risk assessment in 6-7-year-old Chinese children. J Dent. 2006;34:676-81.

Åkerblom A, Rohlin M, Hasselgren G. Individualised restricted intraoral radiography versus full-mouth radiography in the detection of periradicular lesions. Swed Dent J. 1988;12:151-9. 
I 

Review article

\section{Diagnostic accuracy of different caries risk assessment methods. A systematic review}

\section{Anna Senneby ${ }^{a}$, Ingegerd Mejàre ${ }^{b}$, Nils-Eric Sahlinn ${ }^{c}$, Gunnel Svensäter ${ }^{a}$,} Madeleine Rohlin ${ }^{\mathrm{a}, *}$

a Faculty of Odontology, Malmö University, 20506 Malmö, Sweden

The Swedish Council on Technology Assessment in Health Care, Box 3657, 10359 Stockholm, Sweden

'Department of Medical Ethics, Lund University, 22184 Lund, Sweden

A R T I C LE IN FO

Article history:

Received 28 April 2015

Received 28 April 2015 Accepted 15 October 2015

\section{Keywords:}

Sensitivity

Specificity

Likelihood ratio

Microbiota

Dental caries

QUADAS-2

\section{A B S T R A C T}

Objectives: To evaluate the accuracy of different methods used to identify individuals with increased risk of developing dental coronal caries.

Data: Studies on following methods were included: previous caries experience, tests using microbiota, buffering capacity, salivary flow rate, oral hygiene, dietary habits and sociodemographic variables. QUADAS-2 was used to assess risk of bias. Sensitivity, specificity, predictive values, and likelihood ratios (LR) were calculated. Quality of evidence based on $>3$ studies of a method was rated according to GRADE. Sources: PubMed, Cochrane Library, Web of Science and reference lists of included publications were Sources: PubMed, Cochrane 2 searched up to January 2015 .
seaty

searched up to January 2015.
Study selection: From 5776 identified articles, 18 were included. Assessment of study quality identified Study selection: From 5776 identified articles, 18 were included. Assessment of study quality identified
methodological limitations concerning study design, test technology and reporting. No study presented low risk of bias in all domains. Three or more studies were found only for previous caries experience and salivary mutans streptococci and quality of evidence for these methods was low. Evidence regarding other methods was lacking. For previous caries experience, sensitivity ranged between 0.21 and 0.94 and specificity between 0.20 and 1 . Tests using salivary mutans streptococci resulted in low sensitivity and hecificity between 0.20 and 1 . Tests using salivary mutans streptococci resulted in low sensitivity and
previous caries experience and 4 for salivary mutans streptococci, given a threshold $\geq 10^{5} \mathrm{CFU} / \mathrm{ml}$. previous caries experience and 4 for salivary mutans streptococci, given a threshold $\geq 10^{5} \mathrm{CFU} / \mathrm{ml}$.
Conclusions: Evidence on the validity of analysed methods used for caries risk assessment is limited. As methodological quality was low, there is a need to improve study design.

Clinical significance: Low validity for the analysed methods may lead to patients with increased risk not being identified, whereas some are falsely identified as being at risk. As caries risk assessment guides individualized decisions on interventions and intervals for patient recall, improved performance based on best evidence is greatly needed.

(C) 2015 Elsevier Ltd. All rights reserved.

\section{Introduction}

To increase the effectiveness of preventive interventions, it is necessary to identify individuals not at risk and those with increased risk for future caries development. For those not at risk, redundant examinations and interventions will be reduced. When individuals at risk are effectively identified, resources and interventions can be directed towards those in need and they can be better cared for. This is reflected in an increasingly emphasis

\footnotetext{
* Corresponding author at: Malmö University, Faculty of Odontology, SE-205 06 Malmö, Sweden.
}

E-mail address: madeleine.rohlin@mah.se (M. Rohlin). on the importance of prevention and early non-invasive intervention, the aim of which is to prevent progression of a caries lesion. Moreover, the prevention given in risk categories is to be proportional to the estimated risk. Thus, one can hope to optimize resource allocation at both the individual and societal level.

Previous caries experience, based on visual and tactile examinations, is commonly used for caries risk assessment performed in everyday clinical practice. To increase diagnostic accuracy of the detection of approximal caries lesions, radiography is added in some dental clinics. Risk assessment using previous caries experience is a widely implemented method since it is combined with the detection of caries lesions and does not require any additional materials in the clinic. The addition of radiography is seldom applied in scientific studies on risk assessment methods, 
thus contributing to uncertainties surrounding the results of risk assessment performed with the aid of previous caries experience as a method. Other methods used for caries risk assessment are based on microbiological tests, including numbers of mutans streptococci and lactobacilli in the oral cavity. Other approaches, such as oral hygiene, frequency of carbohydrate intake, salivary flow rate and buffer capacity, are on an individual level. A sociodemographic perspective e.g. parents' education and ethnic background, has also been put forward for consideration when identifying individuals with increased caries risk. In addition, multifactorial models that embrace several methods/tests, such as the Cariogram, are used in clinical practice. In general, study results on caries risk assessment are heterogeneous and indicate variations with respect to their predictive potential with the risk for misinterpreting the clinical value when identifying individuals with increased risk for caries [1]. An appraisal of four caries risk assessment systems/guidelines identified that evidence on the validity among such systems is limited and that collecting information on a large number of factors is not more accurate than collecting information on just a few [2].

Systematic reviews have proven useful to summarize and assess applied health research-not just for weighing existing evidence but also for identifying knowledge gaps. Compared with systematic reviews of therapeutic interventions, reviews of diagnostic tests accuracy carry additional complexities. To tackle these complexities, several advances in review methodology have been made in recent years. Since its publication, the QUADAS (Quality Assessment of Diagnostic Accuracy Studies) tool [3] has been widely used. The QUADAS-2 is an improved, redesigned tool that is based on both experience using the original tool and new evidence about sources of bias and variation in diagnostic accuracy studies [4]. To the best of our knowledge, the present review is among the first in dentistry to implement QUADAS-2-while at the same time, paying careful attention to other methodological issues developed for systematic reviews presented in the Assessment of Multiple Systematic Reviews (AMSTAR) [5] and the Preferred Reporting Items for Systematic reviews and Meta-Analyses (PRISMA) Statement [6].

Several factors threaten the validity of a study of diagnostic accuracy. Some of these factors are related to the study design, the selection of patients and the analysis of data. Differences in the execution of the method and reference standard are potentia sources of variation in diagnostic accuracy. Therefore carefu assessment of the quality of included studies is essential for systematic reviews of diagnostic accuracy studies. The aim of this systematic review is to analyse the diagnostic accuracy of different methods used to identify individuals with increased risk of dental coronal caries. Specifically, we will analyse the execution of the reported methods and reference standard thoroughly and propose details to facilitate improvements of future studies of caries risk assessment.

\section{Materials and methods}

To achieve a systematic approach, we conducted the review in accordance with the PRISMA Statement [6] and CRD's guidance for undertaking reviews in health care [7].

\subsection{Problem specification}

What is the diagnostic accuracy of different methods used for identifying individuals with increased risk of dental corona caries?

In this context we defined risk as the probability of an unwanted (negative) event. The event is not certain but may or may not occur. It is a risk for the patient, i.e. we considered dental coronal caries as a negative outcome. Risk of dental caries was THEREFORE defined as "The development either (i) from sound tooth surface to detectable lesion in enamel or dentin: i.e., from health to disease or (ii) from detectable to more extensive lesion: i.e., from disease to more severe disease.

\subsection{Literature search}

The plan for the search was conducted with the aid of university librarians. Three databases were searched: PubMed, the Web of Science and the Cochrane Library in Cochrane Database of Systematic Reviews, updated to January 2015. The searches are summarized in Appendix Table 1. The Web of Science search was performed in all citation databases. The PubMed and Web of Science searches were screened for duplicate publications using a reference management program and by manual search. In addition, the reference lists of included publications and systematic reviews were screened for additional publications, which were considered for inclusion.

\subsection{Publication retrieval}

Two authors independently reviewed the retrieved titles and abstracts. When at least one reader identified a publication as matching the problem specification, it was ordered in full-text. Then, two authors (AS, MR) independently reviewed all complete texts and included or excluded them using a piloted protocol with criteria formulated in accordance with CRD's guidance for undertaking reviews in health care [7]:

Population: all age groups, attrition $<40 \%$, caries prevalence at baseline expressed, or possible to calculate, as percentage of total sample.

Study design: primary study with a prospective and longitudinal design or systematic review, follow-up time $\geq 1$ year.

Methods/criteria for assessing caries: described to permit replication. When no explicit methods/criteria were presented but were referred to, the referenced publication was retrieved and read to find key information.

Index test: clinically applicable, i.e. can be used in a general dental clinic. Described to permit replication, with thresholds to allow calculation of diagnostic accuracy.

Reference standard: caries increment/incidence/experience, described to permit replication with thresholds to allow calculation of diagnostic accuracy.

Outcome: data required to populate $2 \times 2$ tables cross-classifying index test and reference standard to enable calculation of sensitivity, specificity, predictive values and likelihood ratios with confidence intervals.

Language: English.

\subsection{Quality assessment and data synthesis}

Quality assessment was conducted by three authors independently (AS, MR, GS) using the QUADAS-2 tool [4] for risk of bias (low, high or unclear) in four domains: (i) sample selection, (ii) index test, (iii) reference standard, and (iv) flow and timing. The first three domains were also assessed for concerns regarding applicability. A QUADAS-2 protocol (Appendix Table 2) was tailored to the problem specification. The signalling questions were further adapted to the index test analysed and supplemented with items from guidelines for assessing the quality of prognostic studies [8]. Disagreement on the assessments was resolved by consensus. Studies assessed to present high risk of bias concerning the index test or reference standard were excluded from further analysis.

Two authors (AS, MR) extracted and tabulated the study characteristics of each study; two other authors (IM, GS) then 
checked their work. To facilitate a comparison of results from different studies of salivary mutans streptococci, the values were transferred to counts per ml saliva. Two-by-two $(2 \times 2)$ tables were generated using data derived from the studies. Where diagnostic accuracy estimates were presented without the possibility to generate $2 \times 2$ tables, these estimates were excluded. Based on the $2 \times 2$ tables, sensitivity, specificity, predictive values, and likelihood ratios were calculated, along with their corresponding $95 \%$ confidence intervals.

Pooled estimates were calculated for results of an index test wherever three or more studies showed similar characteristics concerning age group, thresholds, and reference standard. Forest plots were generated using the statistical software Meta-DiSc (version 1.4) [9]. Heterogeneity was assessed visually, using Forest plots, and statistically, using a chi-square test, with $P<0.05$ indicative of heterogeneity across studies.

The quality of evidence based on results of three or more studies was rated in four grades (high, moderate, low, very low) according to GRADE [10]. Limitations in study quality, inconsistency of results across studies, uncertainty about directness of the evidence, imprecise or sparse data and high risk of reporting bias lowered the grade of evidence.

\section{Results}

\subsection{Literature identification}

Fig. 1 presents a flow diagram of the literature identification and reasons for exclusion. $43 \%$ of the full-text publications were excluded due to "Other research question/main topic". Of these, many focused on correlations between caries related factors and the development of caries. In many studies of multifactorial risk assessment methods, such as the Cariogram, no data was available to populate $2 \times 2$ tables.

Of 18 studies included, 13 came from PubMed, 4 from the Web of Science, and one from the reference lists. As the same sample and the same methodology was analysed in two studies [11,12] data from only one study [12] was included in the description and analysis.

\subsection{Study characteristics}

The following methods (index tests) were identified: (i) previous caries experience, (ii) mutans streptococci sampled from saliva or plaque, (iii) lactobacilli sampled from saliva, (iv) buffer

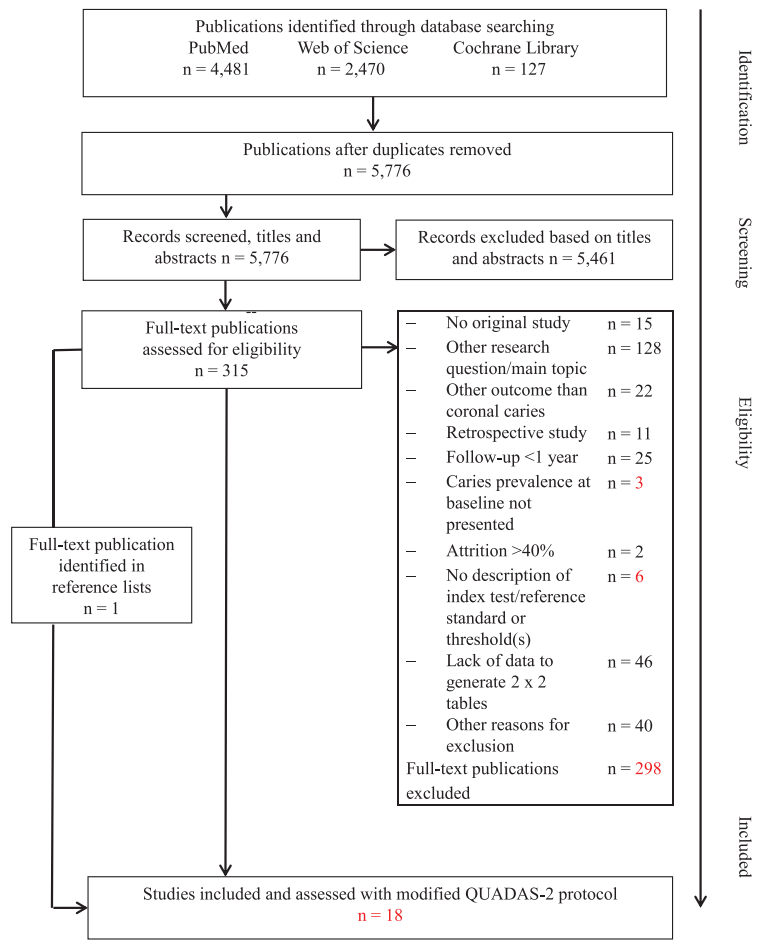

Fig.1. Flow diagram according to the PRISMA Statement [6] presenting study selection process with number of publications retrieved, included, and excluded for systematic review of diagnostic accuracy of different methods used to identify individuals with risk of coronal caries. 
capacity (v) salivary flow rate, (vi) oral hygiene, (vii) dietary habits, and (viii) sociodemographic variables.

The included studies were conducted in 10 countries. Sample size ranged from 27 to 832 individuals. Caries prevalence at baseline - based on cavity in some studies and on enamel and dentinal caries lesions in other - ranged from 0.2 to $83 \%$.

Caries lesions were diagnosed using visual/visual-tactile examination. Four studies added radiography, one fiber-optic transillumination, and one fiber-optic light. The reference standard was phrased as caries increment, caries incidence, caries development, caries experience or new cavities. Caries increment will be used in the following text. Caries increment included enamel and dentinal caries lesions in three studies but only dentinal lesions in the remaining ones. Intra-examiner reproducibility of visual/ visual-tactile examination varied between kappa 0.77 and 0.94 . Pair-wise inter-examiner reproducibility ranged between 0.79 and 0.88 .

Detailed characteristics of included studies of previous caries experience ( $n=12$ [12-23]) are presented in Appendix Table 3 and studies of microbiota sampled from saliva or plaque $(n=10$ [1216,24-28]) in Appendix Table 4, studies of buffer capacity and salivary flow rate $(n=2[12,25])$ and of sociodemographic variables $(n=2[12,16])$ in Appendix Table 5.

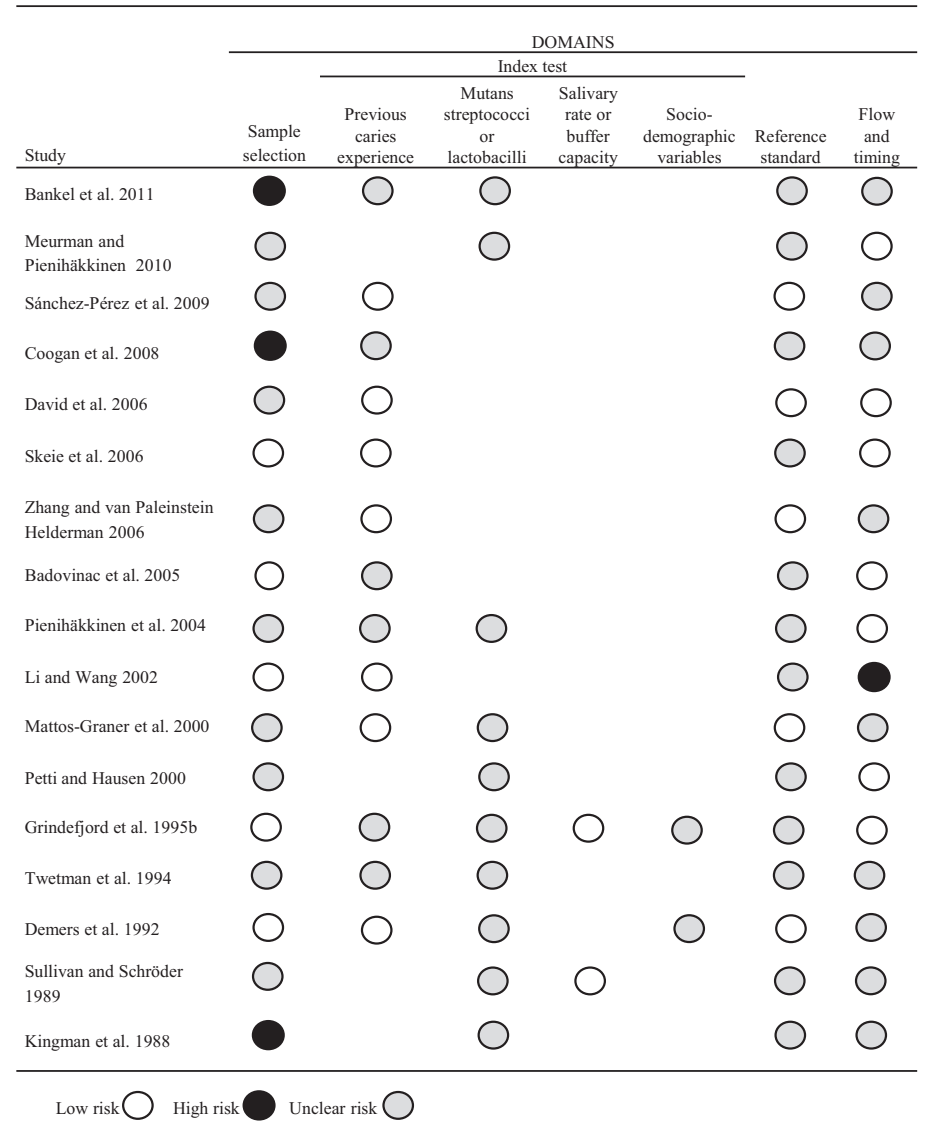

Fig. 2. Risk of bias according to QUADAS-2 [4] for domains: sample selection, index test, reference standard, flow and timing assessed with protocol presented in Appendix Table 2. 


\subsection{Assessment of methodological quality}

As studies on presence of oral hygiene and dietary habits were assessed to present high risk of bias in the index test domain, these studies were excluded from further analysis. Assessment of study quality by QUADAS-2 [4] raised several methodological limitations. No study presented low risk of bias in all domains (Fig. 2). Most studies insufficiently reported the definition of - and, in particular, the rationale for - the criteria for thresholds of the index tests and reference standard. For reference standard, poor reporting of how caries was examined and assessed, resulted in "unclear risk of bias" for most studies. About half the studies were assessed to present unclear risk for flow and timing as withdrawals were not analysed. Concerns regarding applicability were assessed as low except for studies on sociodemographic variables for which it was unclear.

Regarding the domain index test, the studies of microbiota were special as all studies presented unclear risk of bias because their sampling method, examination method, classification system, and method for interpreting results were unclear. Only two studie $[24,26]$ presented the amount of saliva sampled. Information to ascertain detection levels for number of microbiota was insufficient across all studies. All studies used selective MSB-agar, but none used any other growth media to analyse the accuracy of the sampling procedure. It is unclear whether mutans streptococci were identified by their characteristic CFU-morphology as no study offered inclusion/exclusion criteria for their colony morphology. In only two studies $[24,26]$ the growth of mutans streptococci was confirmed by biochemical testing. The highest detectable level of mutans streptococci was not given in any study. Only one study presented examiner reproducibility of the index test [16].

\subsection{Diagnostic accuracy}

Accuracy of each of the 12 studies [12-23] on previous caries experience is presented in Appendix Table 6. Accuracy estimates varied both across studies due to the age of the sample population and different thresholds and within a sample due to different thresholds. Overall, the confidence intervals of sensitivity were wide.

Table 1

Pooled likelihood ratio positive $\left(\mathrm{LR}^{+}\right)$and likelihood ratio negative $(\mathrm{LR}-)$ for previous caries experience and salivary mutans streptococci used to identify children ( $2-7$ years at baseline) with increased risk for coronal caries. Reference-standard (caries increment) threshold: $>0$ caries lesion for included studies. Heterogeneity: $P<0.05$.

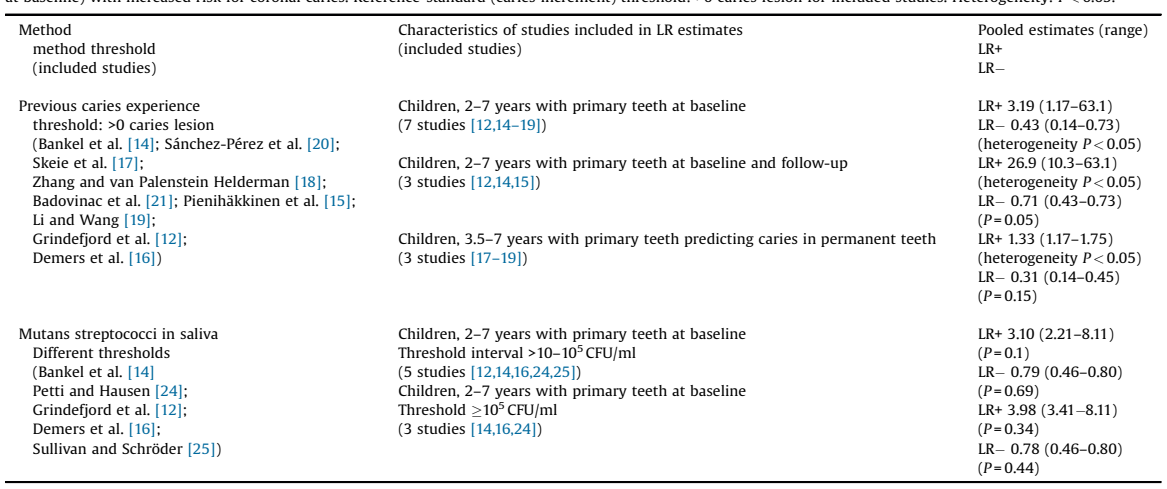

For children aged $2-2.5$ at baseline and primary teeth at followup, pooled sensitivity was low $(0.30)$ and pooled specificity high (0.97), given thresholds of index test and reference standard $>0$ lesion $[12,14,15]$. Pooled LR+ was 26.9 and LR- 0.71 (Table 1 ). There were many false-negative diagnoses but few false-positive.

Predicting caries in permanent teeth based on previous caries experience in primary teeth (children aged 3.5-7) yielded higher pooled sensitivity $(0.91)$ but low pooled specificity $(0.29)$, given index test $\mathrm{dmft}>0$ and reference standard DMFT $>0$ [17-19].Pooled $\mathrm{LR}+$ was 1.33 at a $\mathrm{LR}-$ of 0.31 (Table 1 ). In this sample, false-positive diagnoses were numerous and false-negatives few. For permanent thresholds of caries increment (Fig. 3).

Accuracy of each of the 10 studies [12-16,24-28] on microbiota (mutans streptococci and lactobacilli) sampled from saliva or plaqueis presented in Appendix Table 7. The highest pooled LR+ (3.98) was achieved with threshold $>10^{5} \mathrm{CFU} / \mathrm{ml}$ in children aged 2-7 at baseline (Table 1 ) despite differences in sampling techniques. The influence of different thresholds for the index test is evident in a study of permanent teeth [26] given the same reference standard. Sensitivity ranged between 0.04 for $10^{6} \mathrm{CFU} / \mathrm{ml}$ and 0.75 for $>10^{2} \mathrm{CFU} / \mathrm{ml}$ with corresponding specificity 0.98 and 0.35 (Fig. 4).

For mutans streptococci sampled from plaque and for lactobacilli in children, accuracy estimates were similar to those for salivary mutans streptococci: sensitivity was low and specificity high. A combination of lactobacilli and mutans streptococci did not enhance diagnostic accuracy. Accuracy of studies on salivary flow rate or buffer capacity $[12,25]$ is presented in Appendix Table 8. Diagnostic accuracy of salivary rate was low as was that of buffer capacity (LRs close to 1). Accuracy of studies on sociodemographic variables $[12,16]$ is presented in Appendix Table 8. For mother's or parents' educational level, sensitivity ranged between 0.35 and 0.69 and specificity between 0.57 and 0.77 .

\subsection{Data synthesis}

Data synthesis was performed only for tests using previous caries experience and salivary mutans streptococci. Quality of evidence evaluated according to GRADE [10] was low (Table 2) for previous caries experience and salivary mutans streptococci indicating that further research is very likely to have an important teeth [22] sensitivity varied between 0.21 and 0.62 with different 


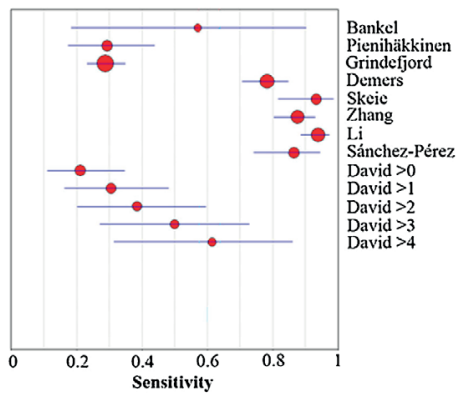

Sensitivity $(0.95 \% \mathrm{CI})$

$0.57(0.18-0.90)$

$0.29(0.23-0.35)$

$0.78(0.71-0.85)$

$0.93(0.82-0.99)$

$0.88(0.80-0.93)$

$0.94(0.89-0.97)$

$0.87(0.74-0.94)$

$0.31(0.16-0.48)$

$0.30(0.27-0.33)$

$0.62(0.32-0.86)$

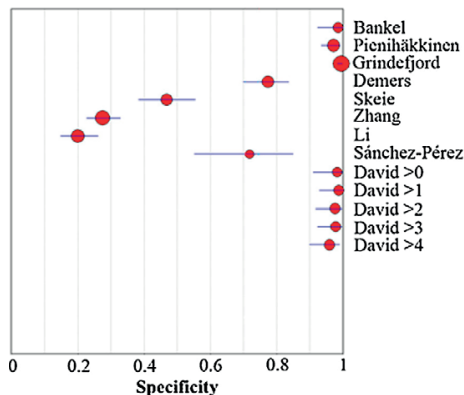

Specificity $(95 \% \mathrm{CI})$

$0.99(0.92-1.00)$

$0.97(0.93-0.99)$

$1.00(0.98-1.00)$

$0.77(0.70-0.84)$

$0.47(0.38-0.55)$

$0.28(0.23-0.33)$

$0.20(0.15-0.26)$

$0.72(0.55-0.85)$

$0.98(0.91-1.00)$

$0.99(0.93-1.00)$

$0.98(0.92-1.00)$

$0.96(0.90-0.99)$

Specificity

Fig. 3. Forest plot presenting estimates of sensitivity and specificity and corresponding $95 \%$ Cls of previous caries experience with threshold $>0$ caries lesion from studies of children aged 2-7 and one study by David et al. [22] of a sample aged 12. Reference-standard (caries increment) threshold was $>0$ caries lesion. As the study of the sample aged 12 reported data for several reference-standard thresholds ( $>0>4$ caries lesions), estimates are included more than once. Size of points reflects total sample size.

impact on our confidence in the estimates. Evidence regarding other tests was lacking as there were too few studies.

As there was significant heterogeneity between studies, presented visually for previous caries experience (Fig. 3) and for salivary mutans streptococci (Fig. 4), no meta-analysis was performed. Clinical heterogeneity arose from differences between sample characteristics e.g. caries severity and prevalence and methodological heterogeneity depended largely on the way index tests and reference standard were conducted and interpreted and different index-test thresholds.

For subgroups of children of the same age-group, there was heterogeneity between studies of previous caries experience, which was not the case between studies of salivary mutans streptococci using the same thresholds (Table 1). Pooled LR+ in children aged 2-7 years and with primary teeth at baseline were 3.19 for previous caries experience and 3.98 for salivary mutans streptococci with threshold $\geq 10^{5} \mathrm{CFU} / \mathrm{ml}$ (Table 1).

\section{Discussion}

4.1. Methodological considerations on this review and study quality

Because diagnostic accuracy studies are often poorly indexed, the resulting search was non-specific resulting in a retrieval of many publications. The review of thousands titles and abstract lead to exclusion of many publications out of which a few may have been incorrectly discarded. Many of the excluded publications investigated correlations between risk factors and caries. A correlation between the index test and reference standard results is a prerequisite for obtaining reasonable diagnostic accuracy but insufficient for analysing diagnostic accuracy and thereby to estimate the potential utility of the test in a clinical setting. The search limitations regarding language and so-called "grey literature" may have resulted in missing publications. Although we conducted a wide search, it is always possible that there may be studies as yet not identified. However, the most effective method of identifying additional relevant studies appears to be reference scanning of included publications [29]. The criteria for inclusion of studies and for QUADAS-2 assessment are open for debate, so some discussion of these is warranted. A follow-up time of one year was considered reasonable given that the progression of caries lesions in primary dentition is relatively rapid $[12,30]$. In permanent dentition, however, a short follow-up time compromises the study, because the progression of carious lesions in permanent teeth has been shown to be slow in general [31]. The smallest sample size was set to 37 individuals, which represents a very small sample. Extreme outcomes - both high and low - are likelier to be found in small samples [32]. Furthermore, it was considered essential that caries prevalence at baseline be stated; diagnostic accuracy may co-vary with prevalence as discussed below.

Our assessment of study quality was hindered in most cases by lack of clear reporting that was uniformly poor with respect to 


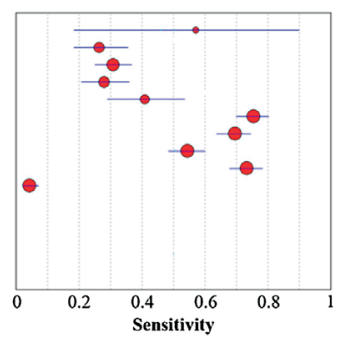

$\begin{array}{lr} & \text { Sensitivity }(0.95 \% \mathrm{C}) \\ \text { Bankel }(>100000) & 0.57(0.18-0.90) \\ \text { Petti }(>500000) & 0.26(0.18-0.36) \\ \text { Grindefjord }(>10) & 0.31(0.25-0.37) \\ \text { Demers }(>100000) & 0.28(0.21-0.36) \\ \text { Sullivan }(>50000) & 0.41(0.29-0.54) \\ \text { Kingman }(>100) & 0.75(0.70-0.80) \\ \text { Kingman }(>100) & 0.69(0.64-0.75) \\ \text { Kingman }(>1000) & 0.54(0.48-0.60) \\ \text { Kingman }(>100000) & 0.73(0.68-0.78) \\ \text { Kingman }(>1000000) & 0.04(0.02-0.07)\end{array}$

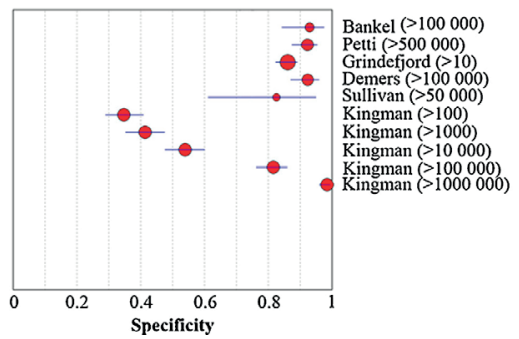

Specificity $(0.95 \% \mathrm{CI})$

$0.93(0.84-0.98)$

$0.92(0.88-0.96)$

$0.86(0.82-0.89)$

$0.92(0.87-0.96)$

$0.85(0.29-0.41)$

$0.41(0.35-0.48)$

$0.54(0.48-0.60)$

$0.82(0.76-0.86)$

$0.82(0.76-0.86)$
$0.98(0.96-1.00)$

Specificity

Fig. 4. Forest plot presenting estimates of sensitivity and specificity and corresponding $95 \% \mathrm{Cls}$ of salivary mutans streptococci with thresholds from $>10$ to $>1,000,000 \mathrm{CFU} /$ $\mathrm{ml}$ from studies of children aged $2-7$ and one study of a sample aged $10-15$ by Kingman et al. [26] As the study of the sample aged $10-15$ reported data for several indexthresholds $(>100->1,000,000 \mathrm{CFU} / \mathrm{ml}$ ), estimates are included more than once. Reference-standard (caries increment) threshold was $>0$ caries lesion. Size of points reflects total sample size.

methods applied, threshold values, examiner reproducibility, and analysis of withdrawals. As emphasized by Hanningan and Lynch [33], sufficient information on the methodology needs to be provided so that a study can be carried out based solely on the information given in a paper. Furthermore, limitations of the methods used for the index tests and reference standard were hardly discussed. Visual/tactile examination was used in most studies on previous caries experience as an index test and in all studies to obtain the reference standard. Although sensitivity of this method is limited and, indeed, insufficient, limitations were not discussed although the risk of misclassification merits further analysis, given how misclassification affects estimates of prevalence and diagnostic accuracy. Examiner reproducibility raises another quality issue; limited reproducibility adversely affects diagnostic accuracy. Many studies failed to report this data.
Intra-examiner agreement, when reported, ranged between kappa 0.77 and 0.94 when detecting caries in primary teeth and pair-wise inter examiner agreement raged between 0.79 and 0.88 . Given that, in most studies, the visual/tactile examinations were performed in daylight, these values seem remarkably high. A systematic review of high and moderate quality studies of caries diagnosis reported corresponding values to range between 0.42 and 0.81 and to be 0.46 , respectively [1]

To improve the methodological quality of research and reporting of tests using microbiota, sampling, culturing and identification procedures and their limitations must be set out clearly. Thus, volume of saliva sampled should be reported to set the lower level of detection for mutans streptococci. Growth medium, allowing total growth of oral bacterial species for control of the sampling procedure should be described. The highest

Table 2

Summary of data and quality of evidence for studies with a reference-standard (caries increment) threshold $>0$ caries lesion for previous caries experience and salivary mutans streptococci used to identify individuals with increased risk of coronal caries. Quality of evidence rated as high, moderate, low or very low and reasons for grade of mutans streptococci used to identify individuals with increased risk of coronal caries. Quality of evidence
evidence applied according to GRADE [10].LR+= likelihood ratio positive; LR-=likelihood ratio negative.

\begin{tabular}{|c|c|c|c|c|}
\hline \multirow{2}{*}{$\begin{array}{l}\text { Method } \\
\text { (threshold) }\end{array}$} & \multirow[t]{2}{*}{ Number of individuals/studies } & \multicolumn{2}{|c|}{ Diagnostic accuracy } & \multirow{2}{*}{$\begin{array}{l}\text { Quality of evidence-Reasons for } \\
\text { lower grade of evidence }\end{array}$} \\
\hline & & $\mathrm{LR}+$ (range) & LR-(range) & \\
\hline $\begin{array}{l}\text { Previous caries experience } \\
\quad(>0 \text { caries lesion })\end{array}$ & $3454 / 11$ & $\begin{array}{l}3.14 \\
(2.06-4.79)\end{array}$ & $\begin{array}{l}0.46 \\
(0.34-0.60)\end{array}$ & Low \\
\hline $\begin{array}{l}\text { Salivary mutans streptococci } \\
\left(>5-10^{5} \mathrm{CFU} / \mathrm{ml}\right)\end{array}$ & $2523 / 6$ & $\begin{array}{l}3.32 \\
(2.21-8.11)\end{array}$ & $\begin{array}{l}0.64 \\
(0.33-0.80)\end{array}$ & $\begin{array}{l}\text { - Limitation of study quality } \\
\text { - Significant inconsistency } \\
\text { - Uncertainty about evidence }\end{array}$ \\
\hline
\end{tabular}


detectable level of mutans streptococci should be given to distinguish 1 from 10 million mutans streptococci per $\mathrm{ml}$ saliva. Dentocult-SM Strip mutans might underestimate mutans streptococci in saliva, since only those mutans streptococci that adhere to and grow on the plastic strip are taken into consideration. In order to validate the growth of mutans streptococci, criteria for colony morphology and/or identification procedure of mutans streptococci should be presented. Otherwise, other species than mutans streptococci capable of growth on MSB-agar, - e.g. Streptococcus mitisand enterococci - may be counted as mutans streptococci.

When it comes to caries increment, only three studies analysed the development from enamel to dentinal caries. When ename caries was included, it was found that considerable proportion of caries increment was due to progression from approximal ename caries to dentinal caries in permanent teeth [22]. Following the philosophy of interceptive prevention, detection of enamel caries lesions is important for reducing the progression of lesions. One can argue that the validity of predictive studies is reduced if enamel lesions are excluded from the caries criteria. Therefore more accurate methods should be used and the inclusion of enamel caries is critical not only to evaluate the method for risk assessment but also to evaluate preventive interventions offered for individuals with increased risk of caries. It should be noted that there is a knowledge gap concerning the effects of non-surgical methods to stop or reverse non-cavitated approximal caries [34].

For many studies, flow and timing were assessed as presenting unclear risk of bias; information on the drop-out group and intervention was insufficient. Readers need to know whether or not patients with characteristics known to affect the results were excluded, which raises the risk of inflated diagnostic accuracy and poses a "limited challenge bias" [35]. Subjects lost to follow-up often differ systematically from those who remain, exemplified in the study by David et al. [22], where the drop-outs had significantly higher baseline caries than the follow-up group.

\subsection{Diagnostic accuracy}

In general, the results of included tests showed low sensitivity. None of the studies described their statistical uncertainty; however, we judged it, using confidence intervals, as high. The underuse of confidence intervals is in line with the results of a review of papers published in dental journals [36]. Estimates on specificity were generally higher, suggesting that it is more certain to identify individuals with low risk of developing caries. Although that likelihood ratios are considered to be more clinically meaningful than other measures [37-39] no study presented this accuracy estimates. Such values have the advantage of incorporating all four cells of the $2 \times 2$ table in contrast to sensitivity and specificity, which make use of only two cells. Likelihood ratios describe how many times likelier a person with the target condition is to receive a certain test result than one without. In the present review, pooled estimates of $\mathrm{LR}+>5$ or $\mathrm{LR}-\leq 0.2$ that is judged to provide strong diagnostic yield [40] were not achieved for any method. Some of the literature [41] on caries prediction claims that sensitivity and specificity are independent of disease prevalence and incidence, but this is contradicted by the results of some included studies in our review $[22,26]$. Furthermore, a systematic review of 17 studies of diagnostic accuracy demonstrated a clear association between disease prevalence and accuracy [42]. As shown in some included studies where prevalence is low, overall accuracy more closely resembles specificity; where prevalence is high, overall accuracy more closely resembles sensitivity in accord with Leeflang et al [43] Therefore a likelihood ratio calculated from a study with a prevalence of $5 \%$ cannot be carelessly used to calculate the posttest probability in a population with a prevalence of $20 \%$ [43].
The present review makes clear that, besides caries prevalence, the thresholds for the index test and the reference standard influence diagnostic accuracy. Consequently, it may be difficult to design a prediction model with a universal index that can apply to all types of populations. Furthermore, the prediction model should be aligned with available health care resources and appropriate interventions offered.

Previous caries experiencehas, typically, been considered "the single best predictor for future caries" (e.g. studies by Demers et al. [16] and van Palenstein Helderman et al. [41]). The likelihood ratios indicate a varied ability with vast differences for different age groups. For two studies $[12,14]$ on children with primary teeth at baseline and follow-up, LR+ estimates were extremely high, but with extreme statistical uncertainty. Contrary to conclusions of previous studies (e.g. Skeie et al. [17]), caries lesions in primary teeth do not accurately predict caries in the permanent teeth according the results of this review with pooled LR+ around 1 . The pooled LR - of 0.3 seems to provide a better estimate, indicating that the odds that increased risk for caries given a negative test result will be low. The fact that the results varied across age groups shows that sub-group analysis of this index test is important.

Microbiotasubjects with the same level of $\mathrm{ms} / \mathrm{ml}$ were classified differently depending on the study: e.g. subjects with $100,000 \mathrm{~ms} /$ $\mathrm{ml}$ were classified at increased risk for caries in four studies $[12,14,16,25]$ and at low risk in two studies $[13,24]$. Kingman et al. [26] alone presented data for several index-test and referencestandard thresholds, making possible sub-groups analyses.

Sampling technique and thresholds aside, using microbiota sampled from saliva or plaque for the index test tended to result in higher specificity than sensitivity: i.e. test results showing few mutans streptococci or lactobacilli seem to be an adequate predictor for lower probability of developing coronal caries. The highest LR+ for mutans streptococci was achieved with the threshold $>10^{5} \mathrm{CFU} / \mathrm{ml}$ saliva despite differing sampling techniques in primary teeth $[14,16,24]$ as well as in permanent teeth [26]. The numerous false negative diagnoses may indicate that bacterial species other than mutans streptococci are involved in caries development. At the same time, several false positives suggest that some individuals seem to harbour numerous mutans streptococci without increased risk for caries. This may support the ecological plaque hypothesis, whereby the total number of aciduric microbiota - rather than the number of mutans streptococci and lactobacilli - is the underlying cause of caries [44].

\section{Conclusions}

Our review reveals an urgent need to improve study design, test technology, and particularly reporting of studies on caries risk assessment. Inadequate reporting can lead to misleading findings with consequences for patient care. There was significant heterogeneity between the results of included studies caused by clinical and methodological issues. Evidence on the validity of analysed methods is limited. The results on diagnostic accuracy are insufficiently robust to determine the comparative accuracy of methods using microbiota vs. that of previous caries experience, though some results suggest comparable estimates expressed as likelihood ratios. Whether previous caries experience is a better predictor than tests using salivary mutans streptococci remains uncertain, particularly for pre-school children. The low LR of both methods highlights a need to develop methods with improved performance and/or a novel approach with new candidate biomarkers.

\section{Conflict of interest}

\section{None.}




\section{Authors contributions}

M. Rohlin, N.-E. Sahlin, G. Svensäter designed the review; A Senneby together with librarians performed the literature search; I. Mejàre, M. Rohlin, A. Senneby, G. Svensäter performed data acquisition, analysis and interpretation, all authors drafted and revised the manuscript.

\section{Acknowledgements}

The review was funded by grants received from The Swedish Dental Association and The Swedish Foundation for the Humanities and the Social Sciences (Riksbankens jubileumsfond) project "Scientific evidence and practical experience". The foundations had no role in study design, data collection, analysis and interpretation of data, in the writing of the report, and the decision to submit the article for publication.

\section{Appendix A. Supplementary data}

Supplementary data associated with this article can be found, in the online version, at http://dx.doi.org/10.1016/j.jdent.2015.10.011.

\section{References}

[1] I. Mejàre, S. Åxelsson, G. Dahlén, I. Espelid, A. Norlund, Å. Svensson, et al., Caries risk assessment. A systematic review, Acta Odontol. Scand. 72 (2014)

[2] M. Tellez, J. Gomez, I. Pretty, R. Ellwood, A.I. Ismail, Evidence on existing caries risk assessment systems: are they predictive of future caries? Commun. Dent. Oral Epidemiol. 41 (2013) 67-78.

[3] P. Whiting, A.W.S. Rutjes, J.B. Reitsma, P.M.M. Bossuyt, J. Kleijnen, The development of QUADAS: a tool for the quality assessment of studies of 10 (2003) 25-48.

[4] P.F. Whiting, A.W. Rutjes, M.E. Westwood, S. Mallett, J.J. Deeks, J.B. Reitsma, et al., QUADAS-2: a revised tool for the quality assessment of diagnostic accuracy studies, Ann. Intern. Med. 155 (2011) 529-536.

[5] B.J. Shea, J.M. Grimshaw, G.A. Wells, M. Boers, N. Andersson, C. Hamel, Development of AMSTAR: a measurement tool to assess the methodological quality of systematic reviews, BMC Med. Res. Methodol. 15 (2007) 10-17.

[6] D. Moher, A. Liberati, J. Tetzlaff, D.G. Altman, PRISMA Group. Preferred reporting items for systematic reviews and meta-analyses: the PRISMA statement, Int. J. Surg. 8 (2010) 336-341. (accessed 1.27.15) www.prismastatement.org.

[7] Centre for Reviews and Dissemination, Systematic Reviews: CRD's Guidance or Undertaking Reviews in Health Care, York Publishing Services Ltd., York 2009. (accessed 10.02.15)

[8] J.A. Hayden, P. Cote, C. Bombardier, Evaluation of the quality of prognosis studies in systematic reviews, Ann. Intern. Med. 144 (2006) 427-437.

[9] J. Zamora, V. Abraira, A. Muriel, K. Khan, A. Coomarasamy, Meta-DiSc a software for meta-analysis of test accuracy data, BMC Med. Res. Methodol. 12
(2006) 31-43. MetaDiSc version 1.4, (accessed 23.02.15) ftp://ftp.hrc.es/pub (2006) $31-43$. MetaDiSc version 1.4, (acces
programas/metadisc/Metadisc_update.htm.

[10] D. Atkins, D. Best, P.A. Briss, M. Eccles, Y. Falck-Ytter, S. Flottorp, et al., Grading quality of evidence and strength of recommendations, Br. Med. J. 328 (2004) 1490 .

[11] M. Grindefjord, G. Dahllöf, B. Nilsson, T. Modéer, Prediction of dental caries development in 1-year-old children, Caries Res. 29 (1995) 343-348.

12] M. Grindefjord, G. Dahllöf, T. Modeer, Caries development in children from 2.5 to 3.5 years of age: a longitudinal study, Caries Res. 29 (1995) 449-454

[13] R.O. Mattos-Graner, D.J. Smith, W.F. King, M.P. Mayer, Water-insoluble glucan synthesis by mutans streptococcal strains correlates with caries incidence in 12- to 30-month-old children, J. Dent. Res. 79 (2000) 1371-1377.

[14] M. Bankel, A. Robertson, B. Köhler, Carious lesions and caries risk predictors in a group of Swedish children 2 to 3 years of age: one year observation, Eur. J.

[15] K. Pienihäkkinen, J. Jokela, P. Alanen, Assessment of caries risk in preschoo children, Caries Res. 38 (2004) 156-162.

[16] M. Demers, J.M. Brodeur, C. Mouton, P.L. Simard, L. Trahan, G. Veilleux, A increment in Montreal children aged 5 years, Commun. Dent. Health 9 (1992) 273-281.
[17] M.S. Skeie, M. Raadal, G.V. Strand, I. Espelid, The relationship between caries in the primary dentition at 5 years of age and permanent dentition at 10 years of age-a longitudinal study, Int. J. Paediatr. Dent. 16 (2006) 152-160.

[18] Q. Zhang, W.H. van Palenstein Helderman, Caries experience variables as indicators in caries risk assessment in 6-7-year-old Chinese children, J. Dent. 34 (2006) 676-681.

[19] Y. Li, W. Wang, Predicting caries in permanent teeth from caries in primary teeth: an eight-year cohort study, J. Dent. Res. 81 (2002) 561-566.

[20] L. Sánchez-Pérez, J. Golubov, M.E. Irigoyen-Camacho, P.A. Moctezuma, E. Acosta-Gio, Clinical, salivary, and bacterial markers for caries risk assessment in schoolchildren: a 4-year follow-up, Int. J. Paediatr. Dent. 19 (2009) 186-192.

[21] R.L. Badovinac, K.E. Morgan, J. Lefevre, S. Wadhawan, L. Mucci, L. Schoeff, et al., Risk assessment criteria applied to a screening exam: implications for improving the efficiency of a sealant program, J. Public Health Dent. 65 (2005) 203-208.

[22] J. David, M. Raadal, N.J. Wang, G.V. Strand, Caries increment and prediction from 12 to 18 years of age: a follow-up study, Eur. Arch. Paediatr. Dent. 7 (2006)

[23] M.M. Coogan, J.M. MacKeown, J.S. Galpin, L.P. Fatti, Microbiological impressions of teeth, saliva and dietary fibre can predict caries activity. J. Dent. (2008) 892-899.

[24] S. Petti, H.W. Hausen, Caries prediction by multiple salivary mutans streptococcal counts in caries-free children with different levels of fluoride

[25] A. Sullivan, U. Schröder, Systematic analysis of gingival state and salivary variables as predictors of caries from 5 to 7 years of age, Scand. J. Dent. Res. 97 (1989) 25-32.

[26] A. Kingman, W. Little, I. Gomez, S.B. Heifetz, W.S. Driscoll, R. Sheats, et al, Salivary levels of Streptococcus mutans and lactobacilli and dental caries experiences in a US adolescent population, Commun. Dent. Oral Epidemiol. 16 (1988) 98-103.

[27] P.K. Meurman, K. Pienihäkkinen, Factors associated with caries increment: a longitudinal study from 18 months to 5 years of age, Caries Res. 44 (2010) 519-524.

[28] S. Twetman, B. Stähl, T. Nederfors, Use of the strip mutans test in the assessment of caries risk in a group of preschool children, Int. J. Paediatr. Dent. 4 (1994) $245-250$

[29] P. Whiting, R. Harbord, L. de Salis, M. Egger, J. Sterne, Evidence-based diagnosis,

J. Health Serv. Res. Policy 13 (Suppl. 3) (2008) S57-63.
[30] A.C. André Kramer, M.S. Skeie, A.B. Skaare, I. Espelid, A.L. Östberg, Caries increment in primary teeth from 3 to 6 years of age: a longitudinal study of

[31] I. Mejàre, H. Stenlund, C. Zelezny-Holmlund, Caries incidence and lesion progression from adolescence to young adulthood: a prospective 15-year

[32] D. Kahneman, Thinking, Fast and Slow, Farrar, Straus and Giroux, New York, 2011.

33] A. Hannigan, C.D. Lynch, Statistical methodology in oral and dental research: pitfalls and recommendations, J. Dent. 41 (2013) 385-392.

[34] I.A. Mejàre, G. Klingberg, F.K. Mowafi, C. Stecksén-Blicks, S.H.A. Twetman, S.H. Tranæus, A systematic map of systematic reviews in pediatric dentistry-what do we really know? Plos One 10 (2015) 1-21 (accessed 16.06.15).

[35] P.M. Bossuyt, J.B. Reitsma, D.E. Bruns, C.A. Gatsonis, P.P. Glasziou, L.M. Irwig. J.G. Lijmer, D. Moher, D. Rennie, H.C.De Vet, Standards for Reporting of Diagnostic Accuracy: towards complete and accurate reporting of studies of diagnostic accuracy: the STARD initiative Standards for Reporting of Diagnostic Accuracy, Clin. Chem. 49 (2003) 1-6.

[36] J.S. Kim, D.K. Kim, S.J. Hong, Assessment of errors and misused statistics in dental research, Int. Dent. J. 61 (2011) 163-167.

[37] D.A. Grimes, K.F. Schulz, Refining clinical diagnosis with likelihood ratios, Lancet 365 (2005) 1500-1505.

[38] H. Honest, K.S. Khan, Reporting of measures of accuracy in systematic reviews of diagnostic literature, BMC Health Serv. Res. 2 (2002) 1-4.

[39] P.F. Chien, K.S. Khan, Evaluation of a clinical test. II: assessment of validity, BJOG Int. J. Obstet. Gynaecol. 108 (2001) 568-572.

[40] J.J. Deeks, Systematic reviews in health care: systematic reviews of evaluations of diagnostic and screening tests, Br. Med. J. 323 (2001) 157-162.

[41] W.H. van Palenstein Helderman, M.A. Van't Hof, C. van Loveren, E. Bronkhorst, Utility technology in the assessment of the cut-off between a negative and

42] P.E. Whiting A.W. Rutjes, M.E. Westwood, S. Mallett OUADAS-2 Steering Group. A systematic review classifies sources of bias and variation in Group. A systematic review classifies sources of bias and variation in
diagnostic test accuracy studies, J. Clin. Epidemiol. 66 (2013) 1093-1104.

[43] M.M. Leeflang, P.M. Bossuyt, L. Irwig, Diagnostic test accuracy may vary with prevalence: implications for evidence-based diagnosis, J. Clin. Epidemiol. 62 (2009) 5-12.

[44] P.D. Marsh, Are dental diseases examples of ecological catastrophes? Microbiology 149 (2003) 279-294. 


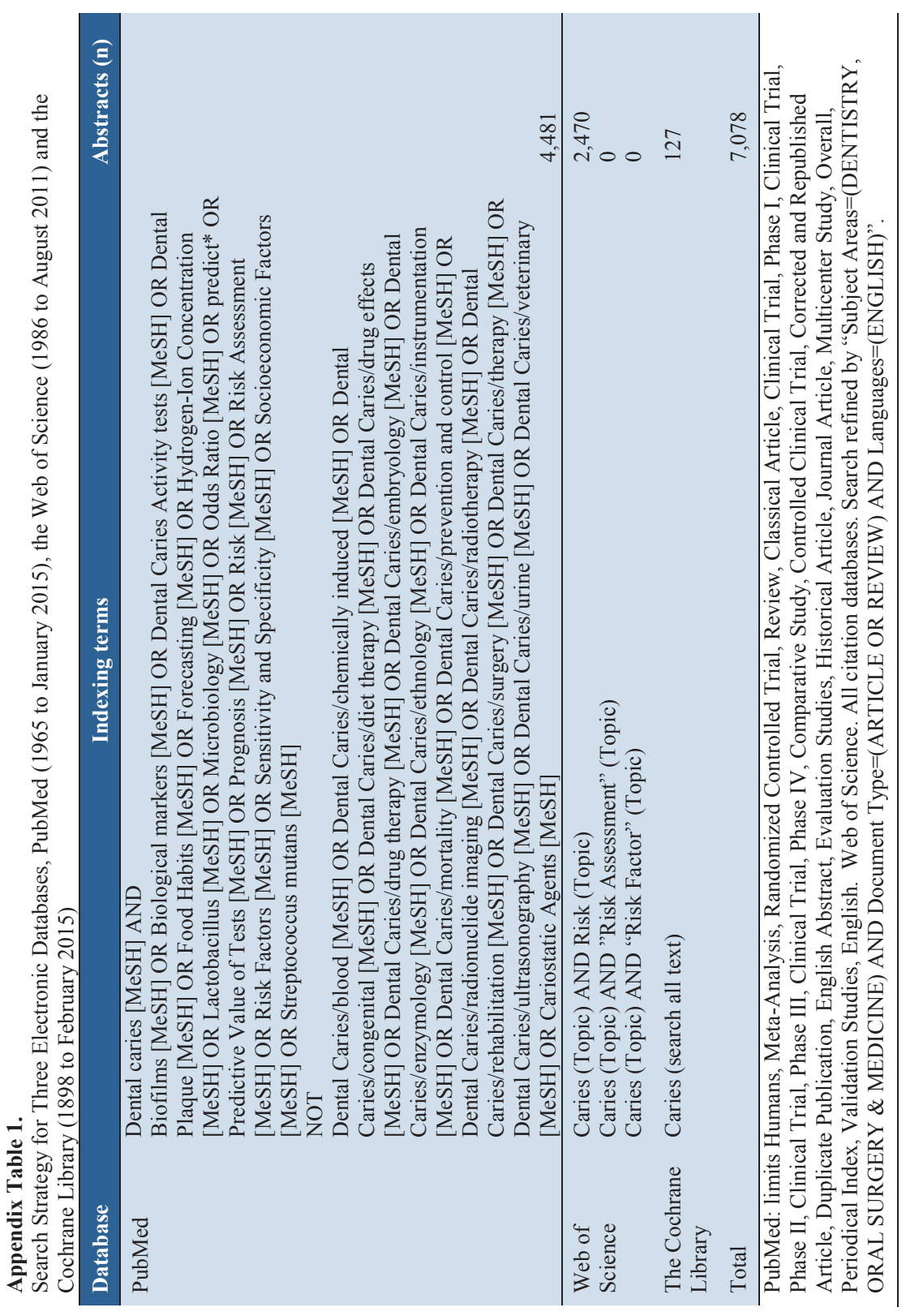




\title{
Appendix Table 2.
}

Modified QUADAS-2 Protocol for Assessment of Potential Risk of Bias and Concerns Regarding Applicability of Studies of Diagnostic Accuracy of Methods for Caries Risk Assessment

\section{A. RISK OF BIAS}

\section{DOMAIN 1: SAMPLE SELECTION}

Describe methods of sample selection

- Source population (population of interest)

- Setting of recruitment

- Method to identify and invite sample

- Inclusion and exclusion criteria

Was a consecutive or random sample of individuals enrolled?

Did the study avoid inappropriate exclusions?

Could the selection of individuals have introduced bias?

RISK of bias

$$
\begin{gathered}
\text { Adequately described } \\
\text { Yes/No/Unclear } \\
\text { Yes/No/Unclear } \\
\text { Yes/No/Unclear } \\
\text { Yes/No/Unclear }
\end{gathered}
$$

Yes/No/Unclear

Yes/No/Unclear

Low/Unclear/High

\section{B. APPLICABILITY}

Describe included individuals:

- Age, number and dentition

- Caries status expressed as number of individuals with and without caries and caries severity

Do the included individuals and setting match the review question? CONCERN regarding applicability

\author{
Adequately described: \\ Yes/No/Unclear \\ Yes/No/Unclear
}

\begin{tabular}{|c|} 
Yes/No/Unclear \\
\hline Low/Unclear/High \\
\hline
\end{tabular}

\section{A. RISK OF BIAS}

\section{DOMAIN 2: INDEX TEST}

Describe the index test and how it was conducted and interpreted:

- Examination method(s) and classification system

- Method of interpretation of results

- Laboratory materials and equipment

Adequately described:

Yes/No/Unclear

Yes/No/Unclear

Yes/No/Unclear

Was the index test adequately described to permit replication?

Yes/No/Unclear Yes/No/Unclear Yes/No/Unclear Was the threshold clearly described?

Was the number of examiner and their experience presented?

Yes/No/Unclear

Was the intra-examiner reproducibility and how it was calculated presented? Yes/No/Unclear Was the inter-examiner reproducibility and how it was calculated presented? Yes/No/Unclear Could the conduct or interpretation of the index test have introduced bias?

$$
\text { RISK of bias }
$$

$$
\text { Low/Unclear/High }
$$

\section{B. APPLICABILITY}

Are there concerns that the index test, its conduct or interpretation differ from the review question?

$$
\begin{array}{ll} 
& \text { Yes/No/Unclear } \\
\cline { 2 - 2 } \text { CONCERN regarding applicability } & \text { Low/Unclear/High } \\
\cline { 2 - 2 } &
\end{array}
$$




\section{Appendix Table 2. \\ (continued)}

\section{DOMAIN 3: REFERENCE STANDARD}

\section{A. RISK OF BIAS}

Describe the reference standard and how it was conducted and interpreted:

- Examination method(s)

- Criteria for caries increment

- Caries increment expressed as sound to caries

- Caries increment expressed as sound to initial and from initial to more severe caries

Adequately described:

Yes/No/Unclear

Yes/No/Unclear

Yes/No/Unclear

Yes/No/Unclear

Was method(s) to obtain the reference standard described to permit replication? Yes/No/Unclear Were the reference standard results interpreted without knowledge of the results of the index test? $\mathrm{Yes} / \mathrm{No} /$ Unclear

Was the threshold pre-specified? Yes/No/Unclear

Was the threshold clearly described regarding number and severity of caries lesions?

Yes/No/Unclear

Was the number of examiner(s) and their experience presented?

Yes/No/Unclear

Was the intra-examiner reproducibility and how it was calculated presented? Yes/No/Unclear

Was the inter-examiner reproducibility and how it was calculated presented? Yes/No/Unclear

Could the reference standard, its conduct or its interpretation have introduced bias?

$$
\text { RISK of bias }
$$

Low/Unclear/High

\section{B. APPLICABILITY}

Are there concerns that the target condition as defined by the reference standard does not match the review question?

Yes/No/Unclear

CONCERN regarding applicability

Low/Unclear/High

\section{A. RISK OF BIAS}

\section{DOMAIN 4: FLOW AND TIMING}

Describe any individuals who did not receive the reference standard or who were excluded from the $2 \times 2$ table:

Describe the time interval and any interventions between index test and reference standard:

Was there any intervention between the index test and reference standard that could influence the caries status compromising the reference standard? Yes/No/Unclear Was sample lost to follow-up analyzed (number, caries prevalence and status)? Yes/No/Unclear Could flow and timing have introduced bias?

$$
\text { RISK of bias Low/Unclear/High }
$$




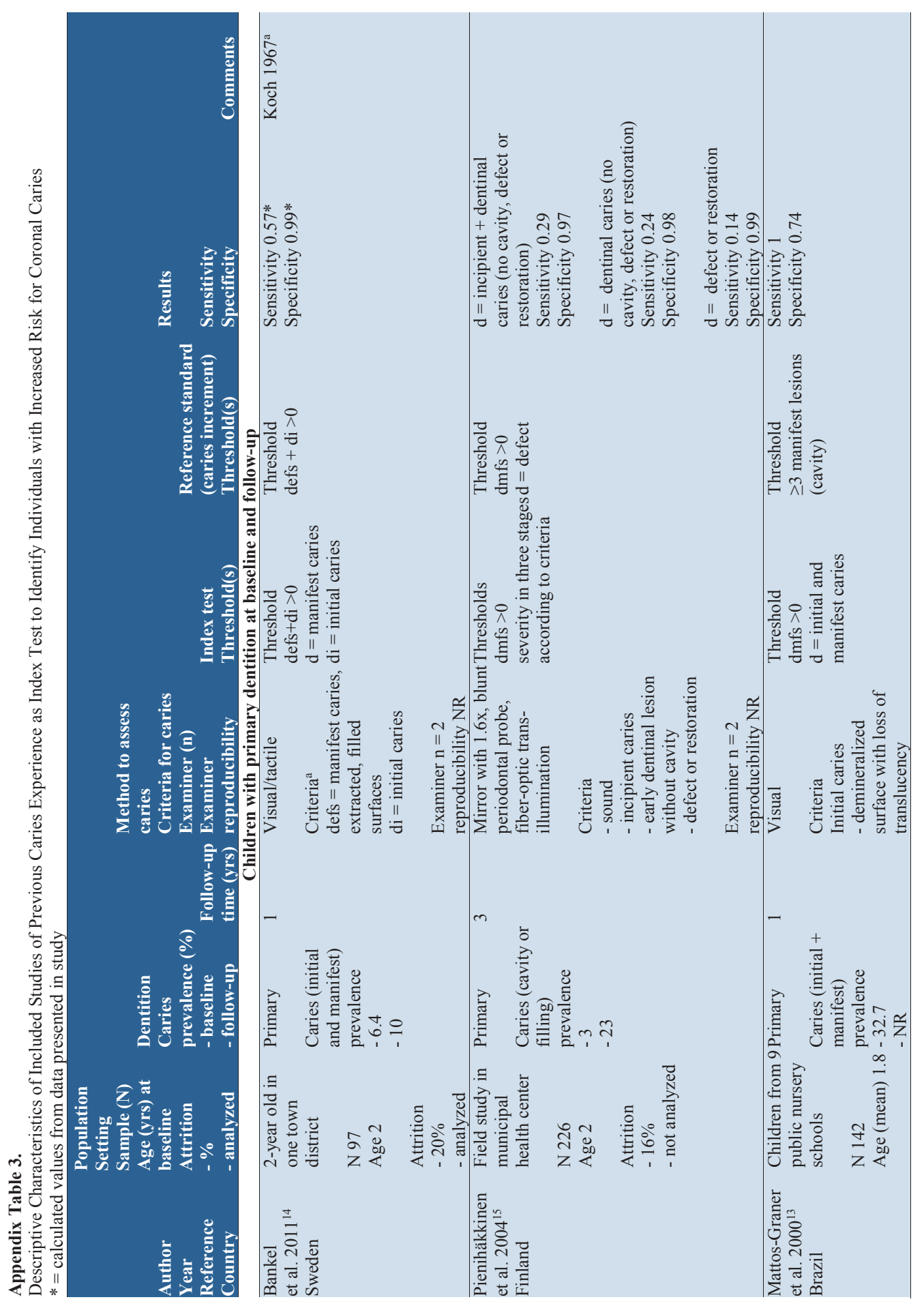




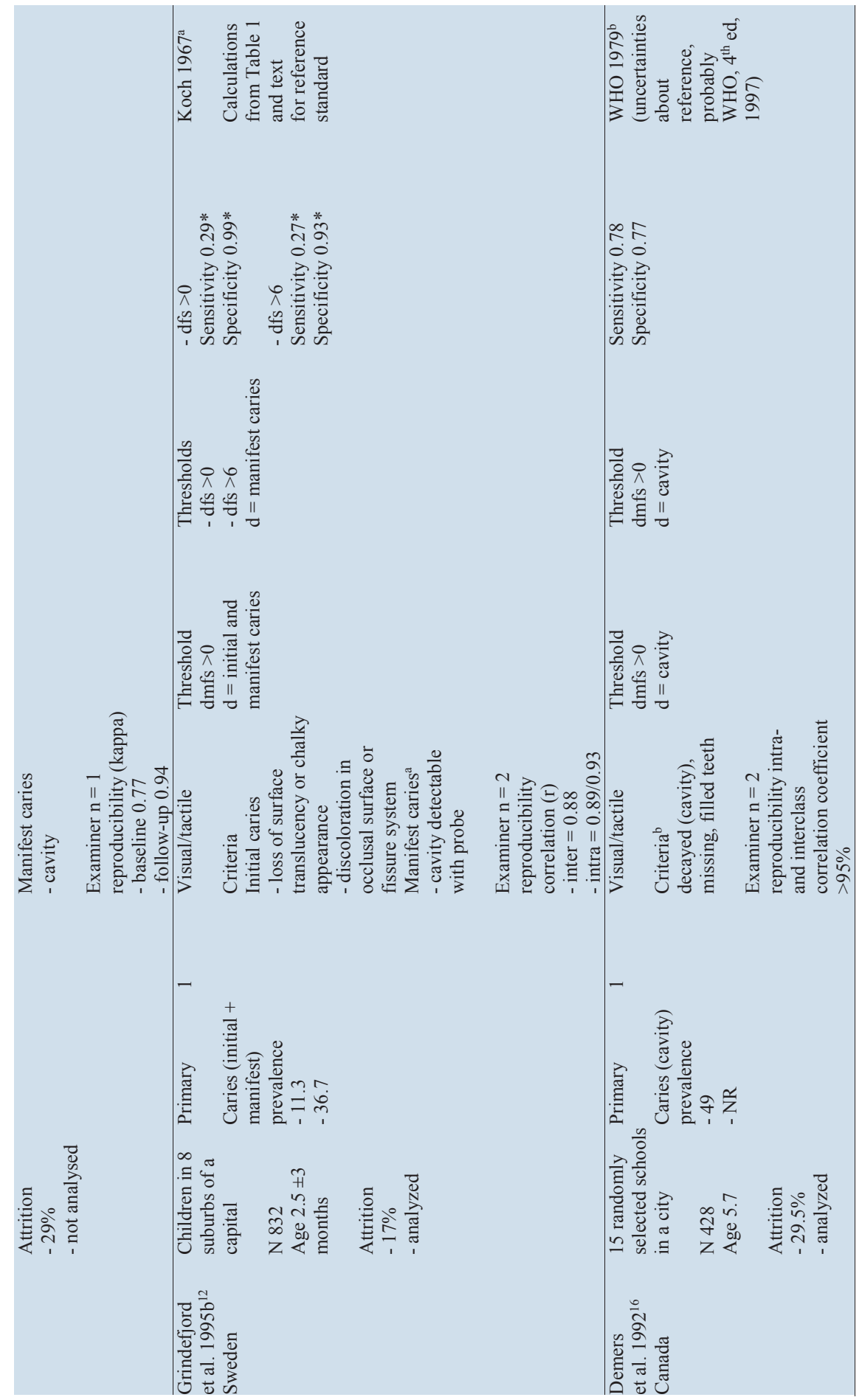




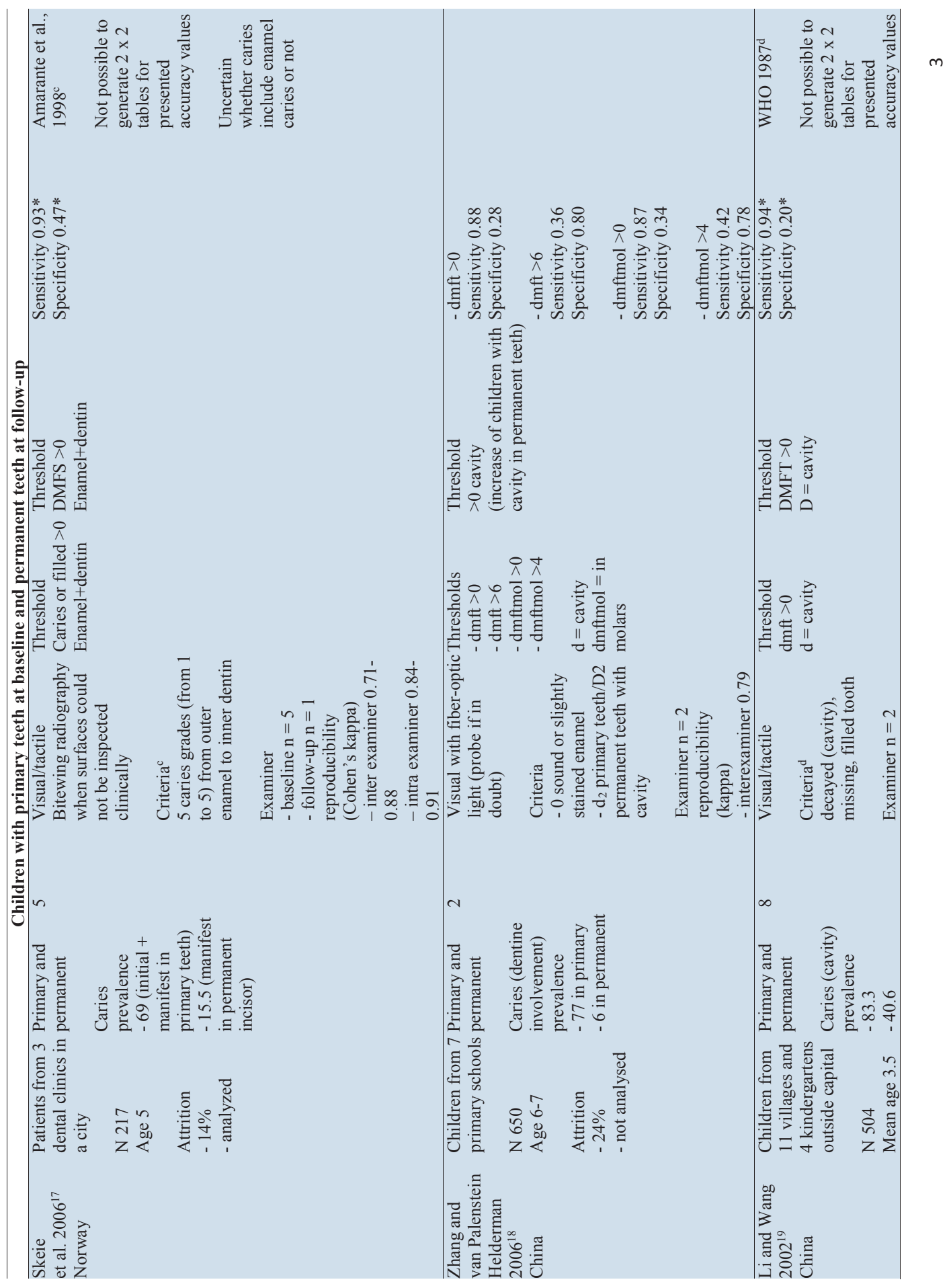




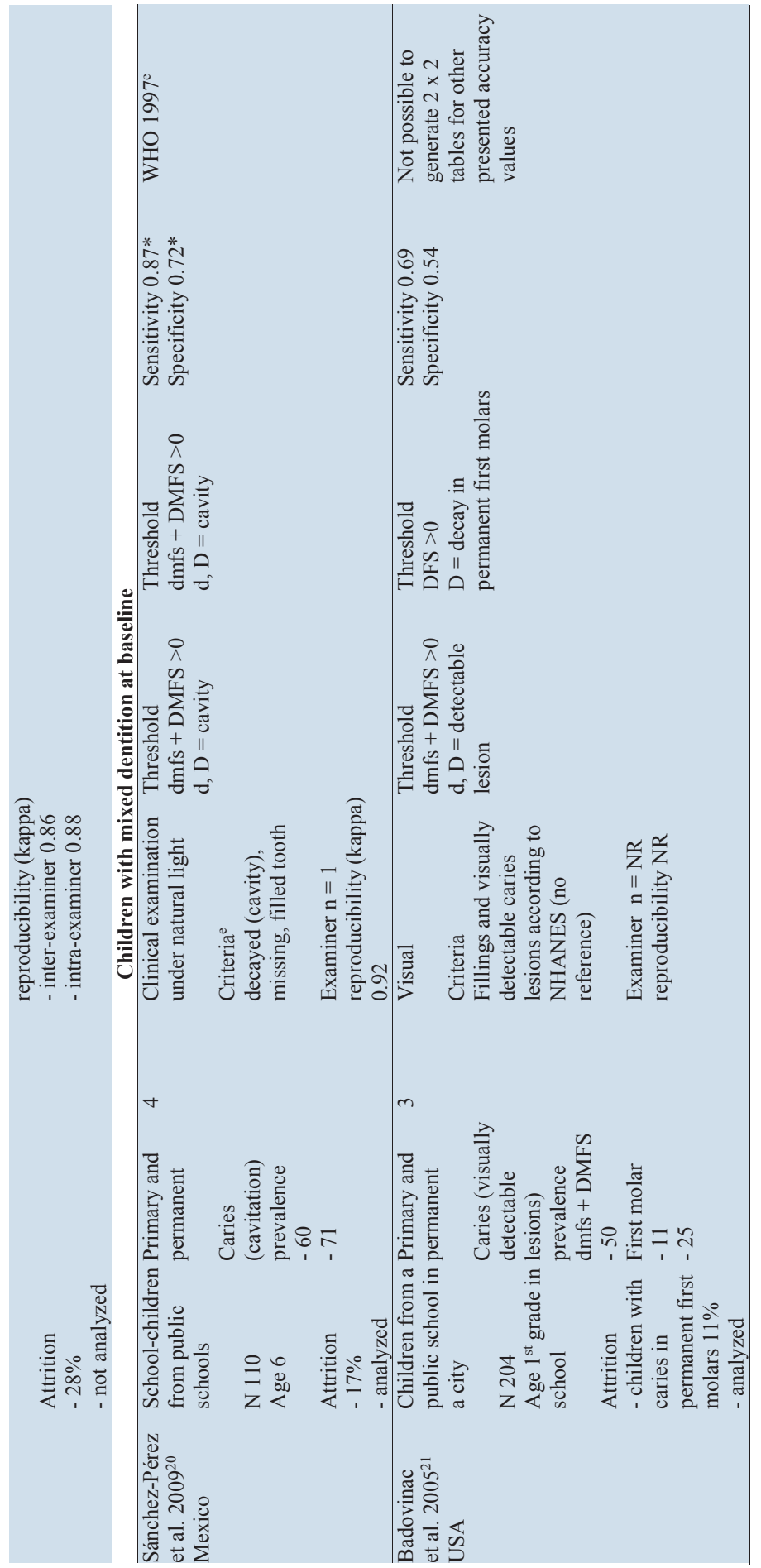




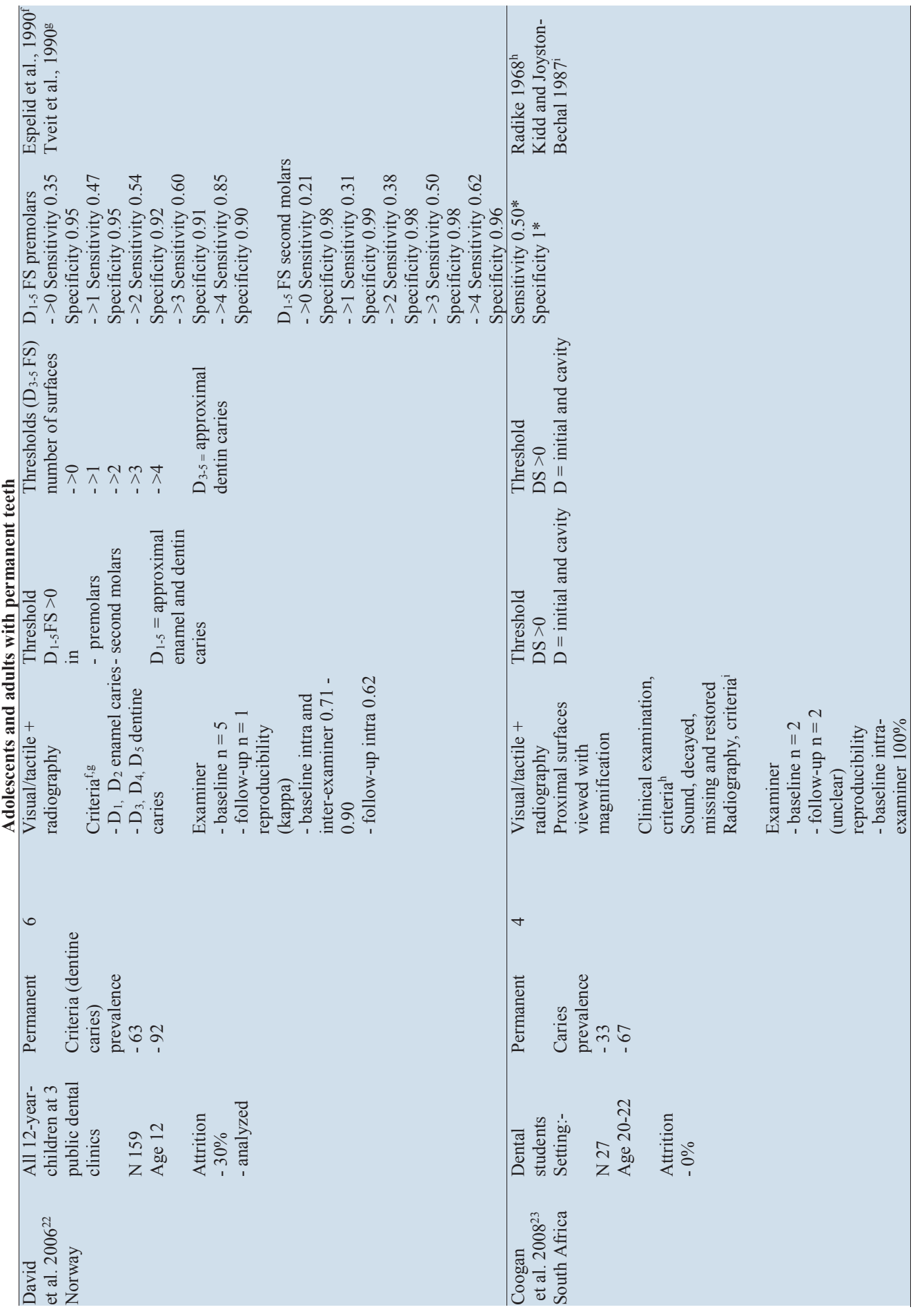




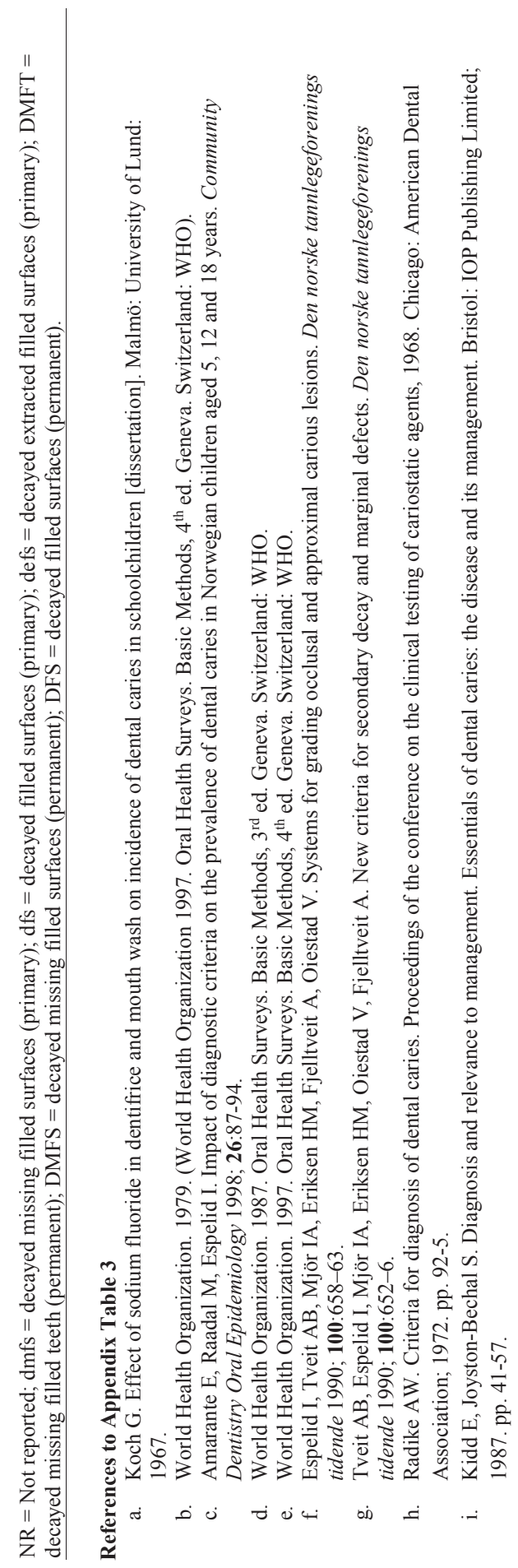




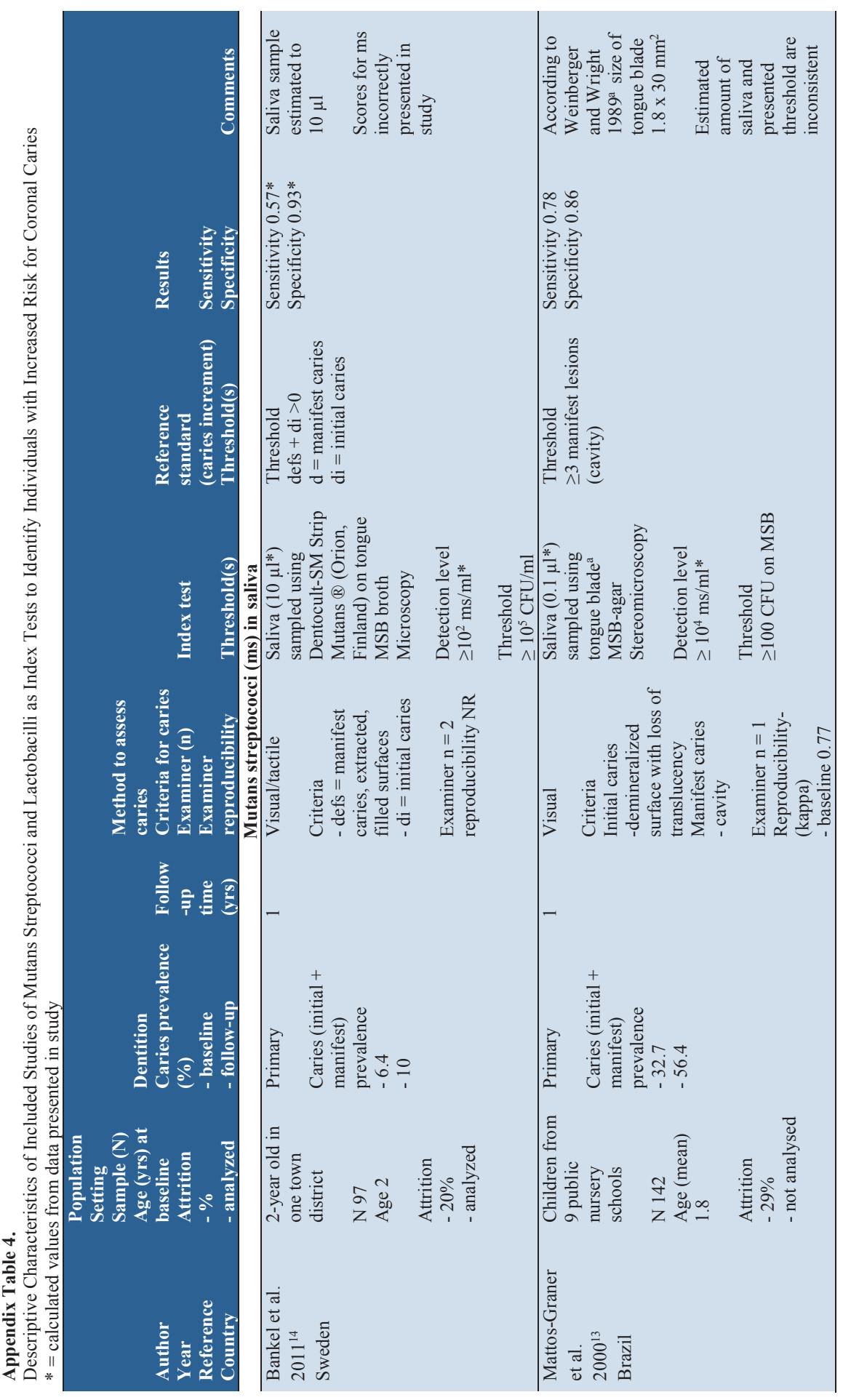




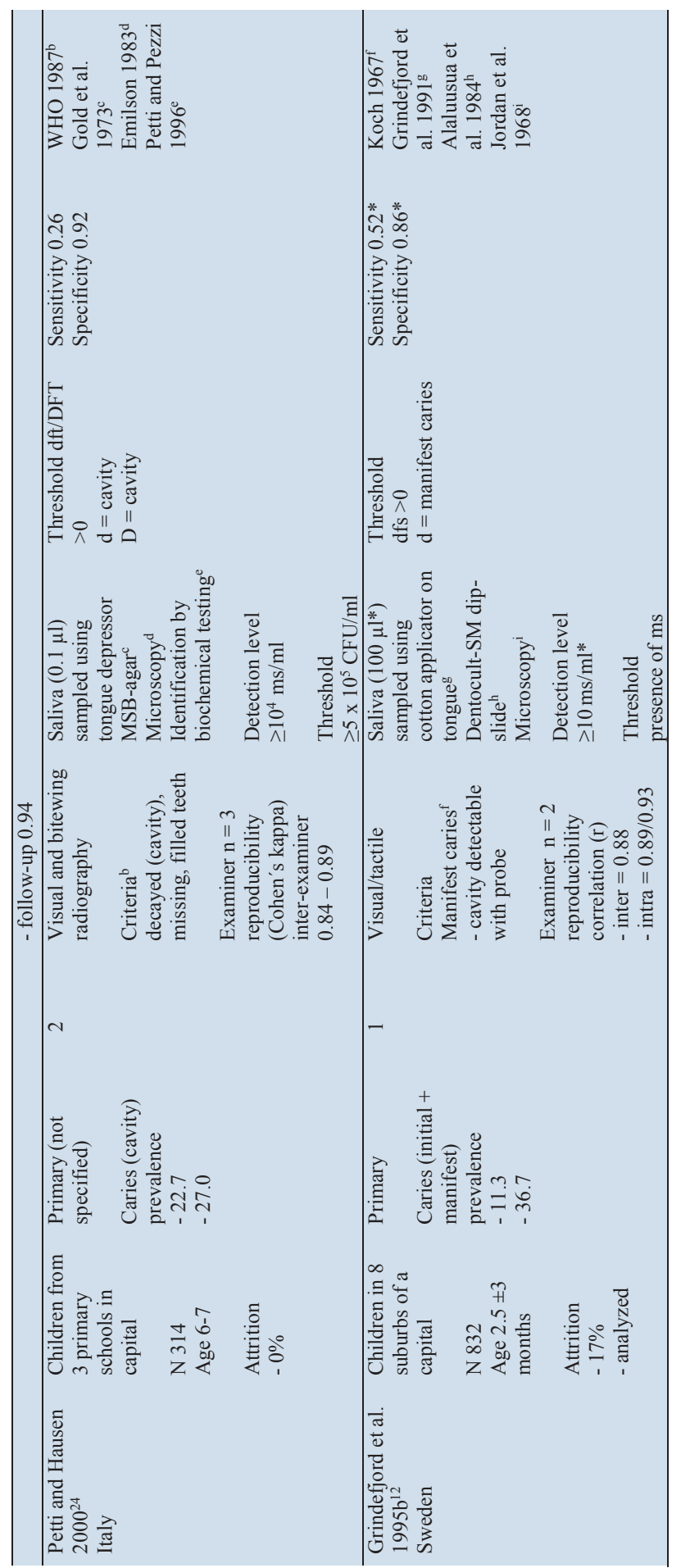




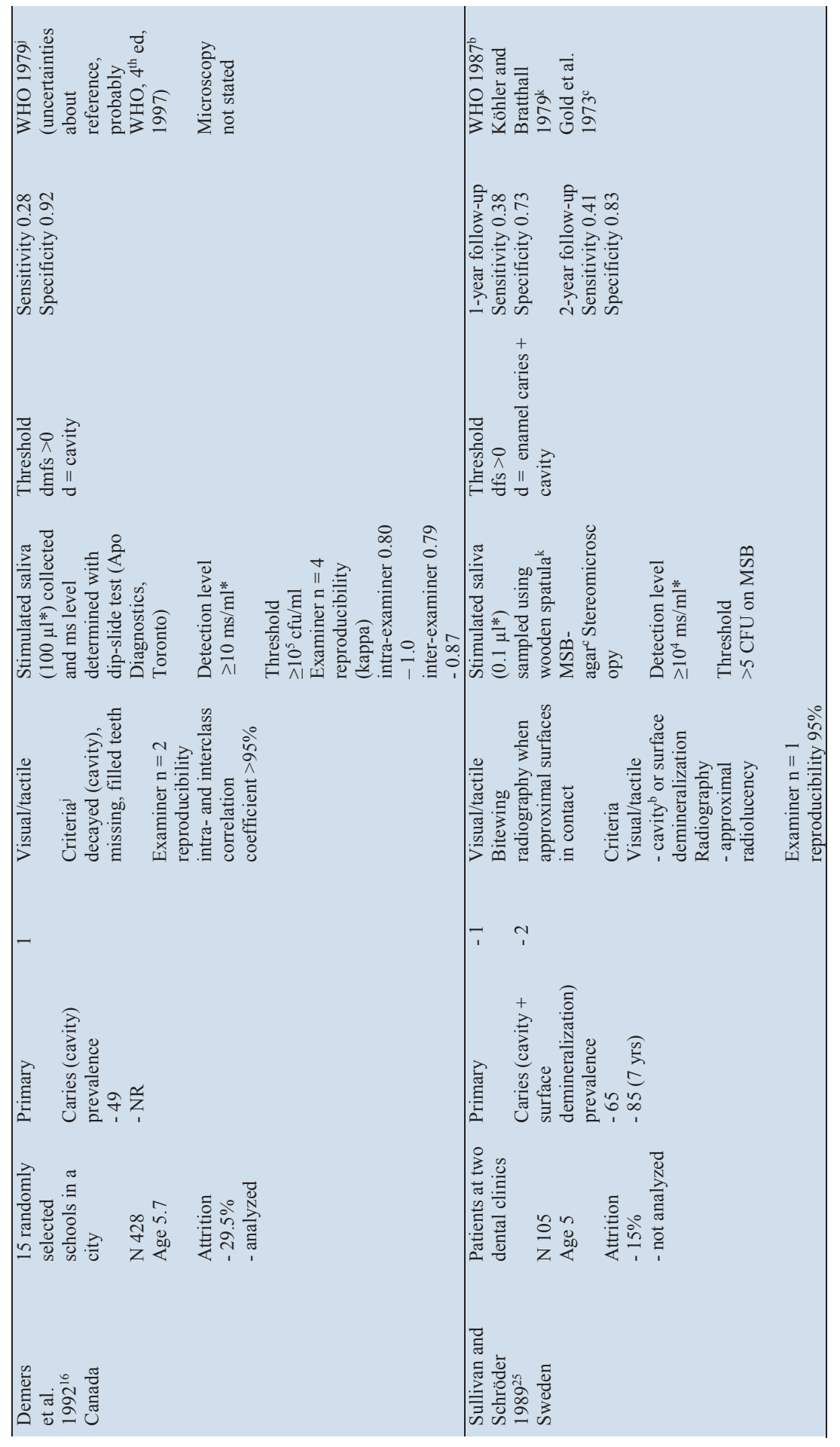


Inn

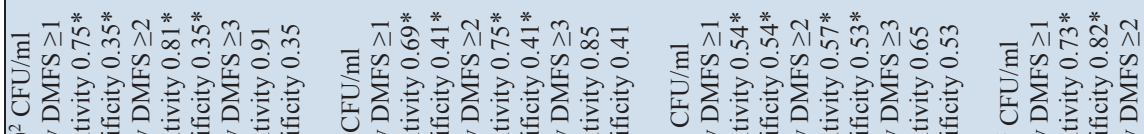

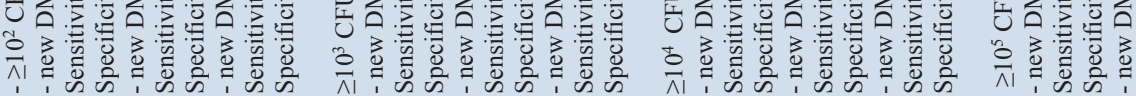

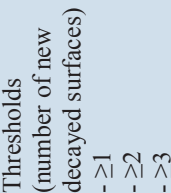

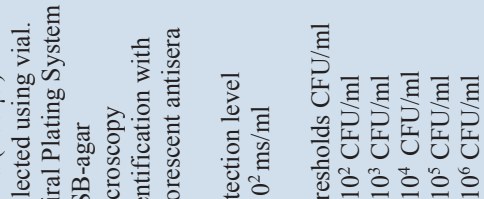

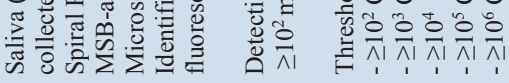

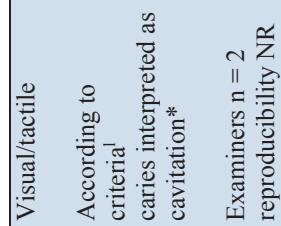

乞

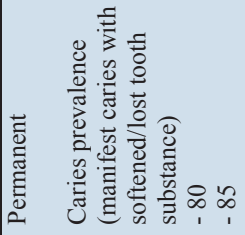

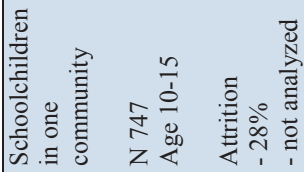

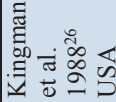




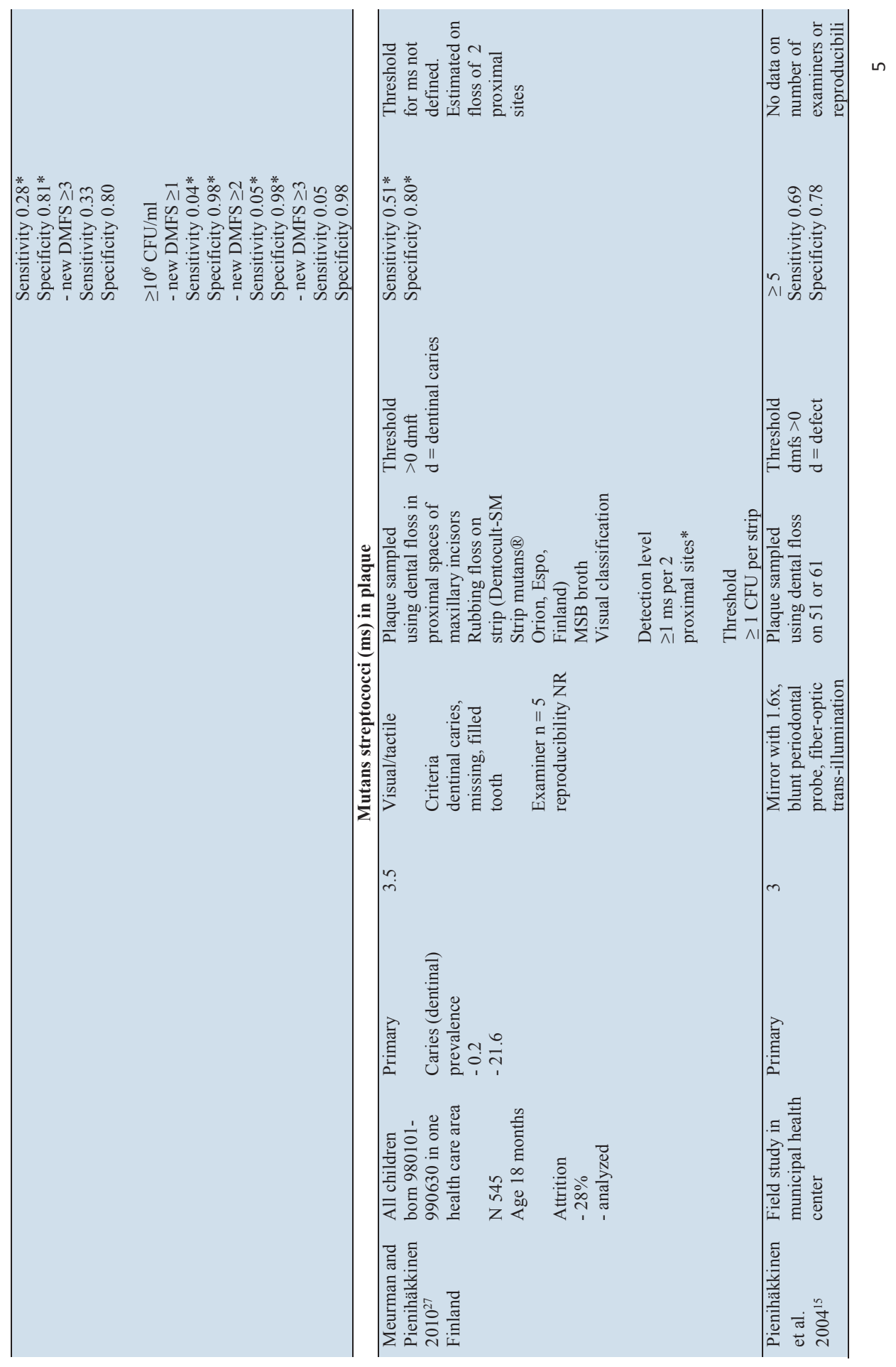




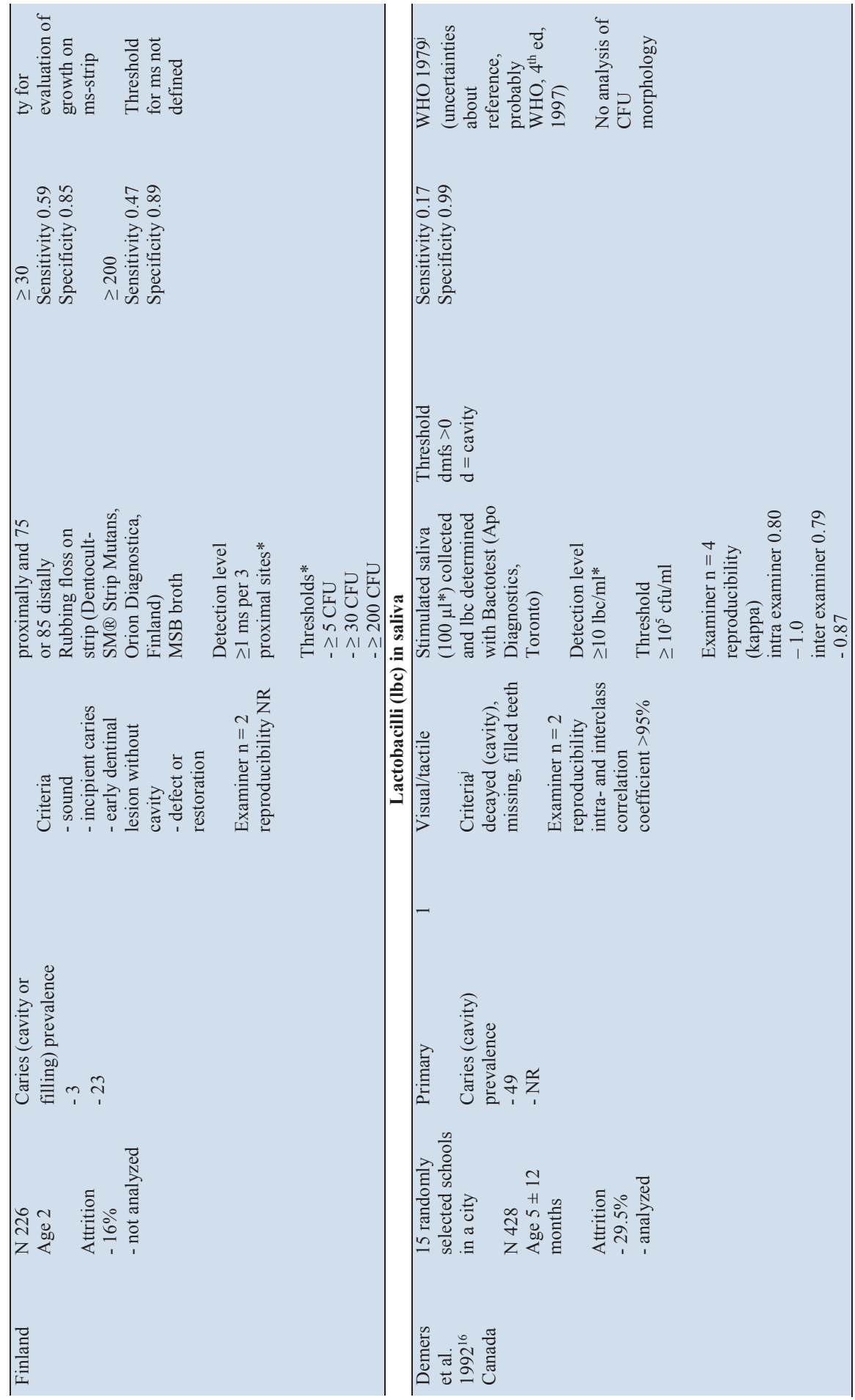




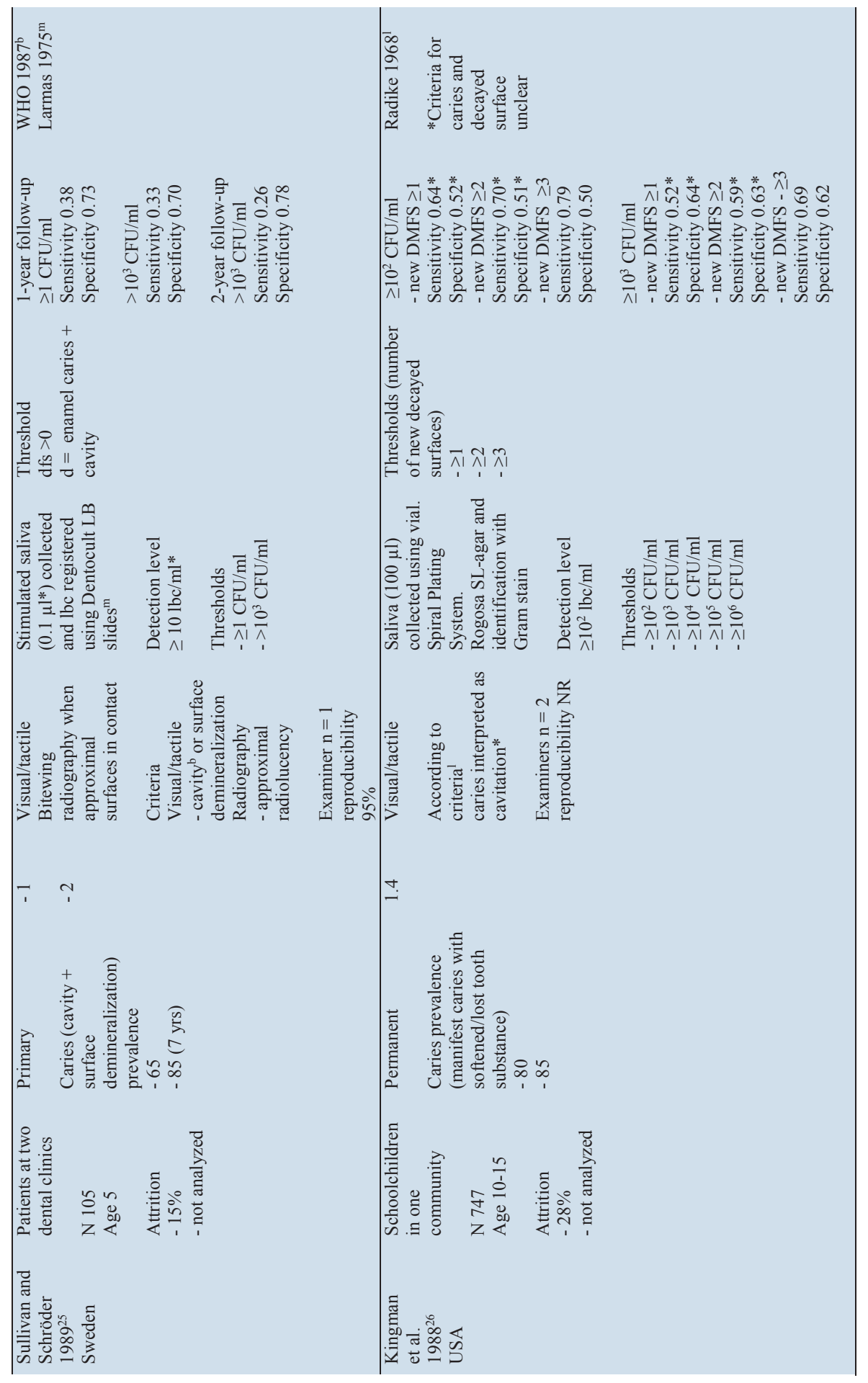




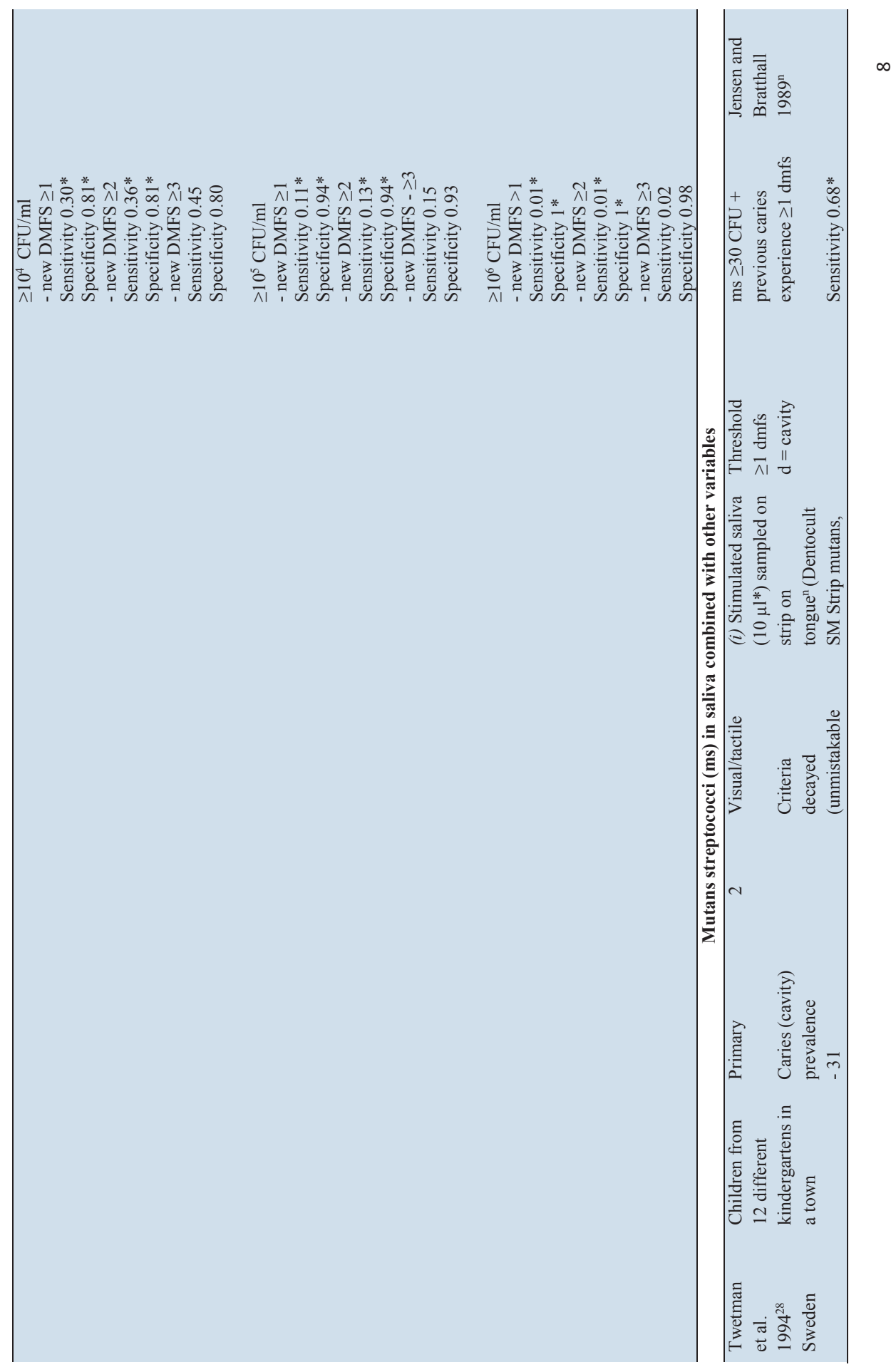




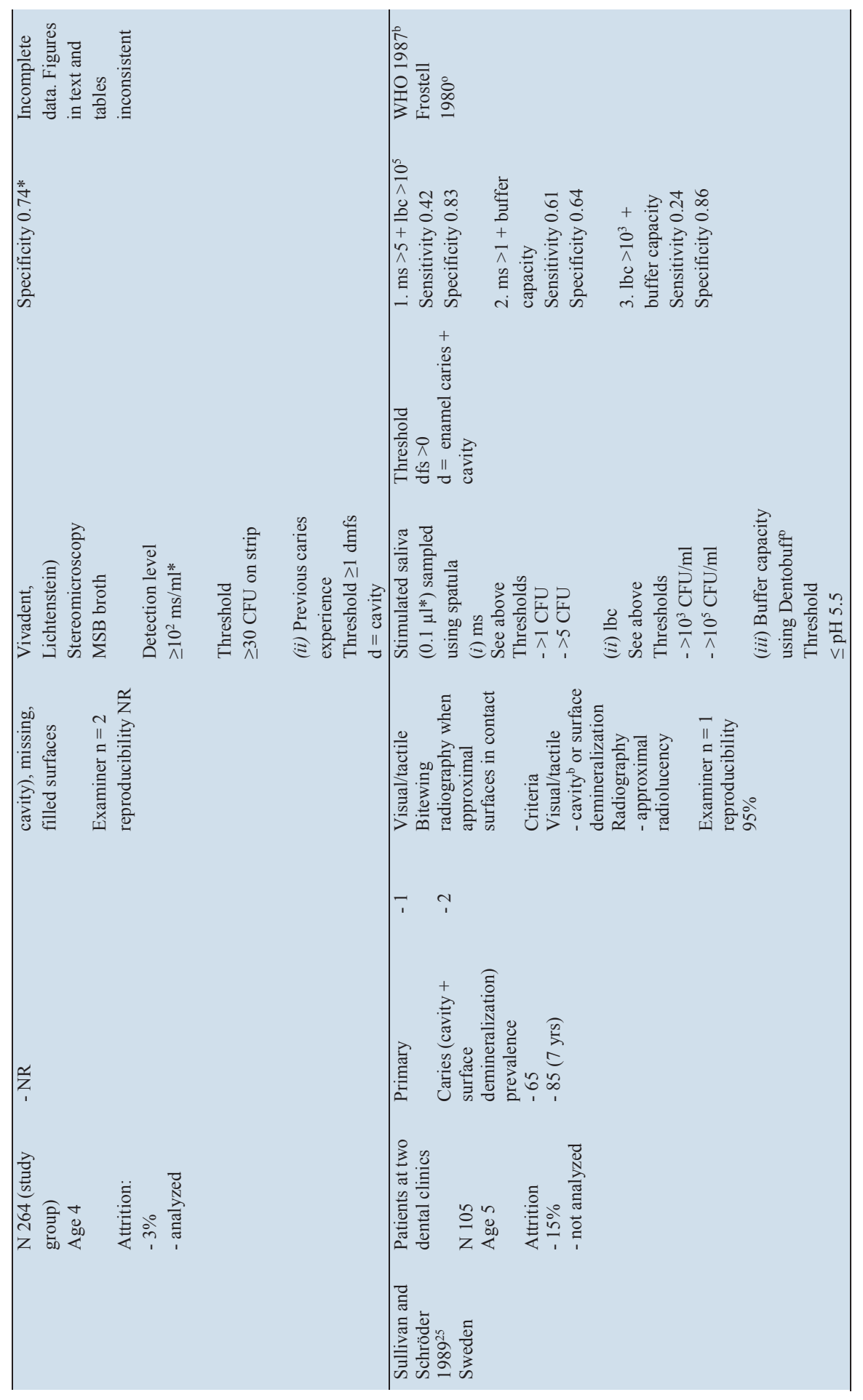




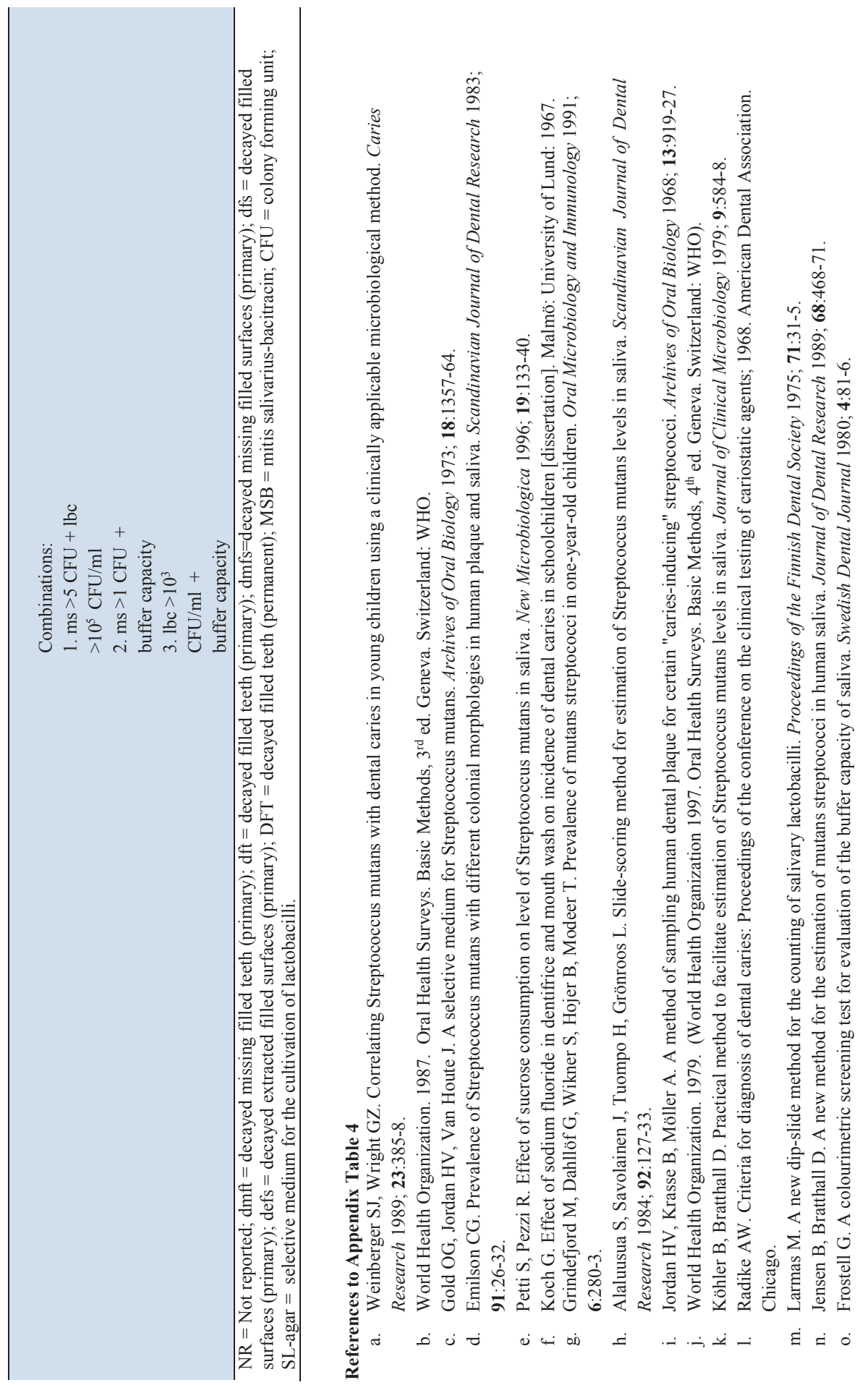




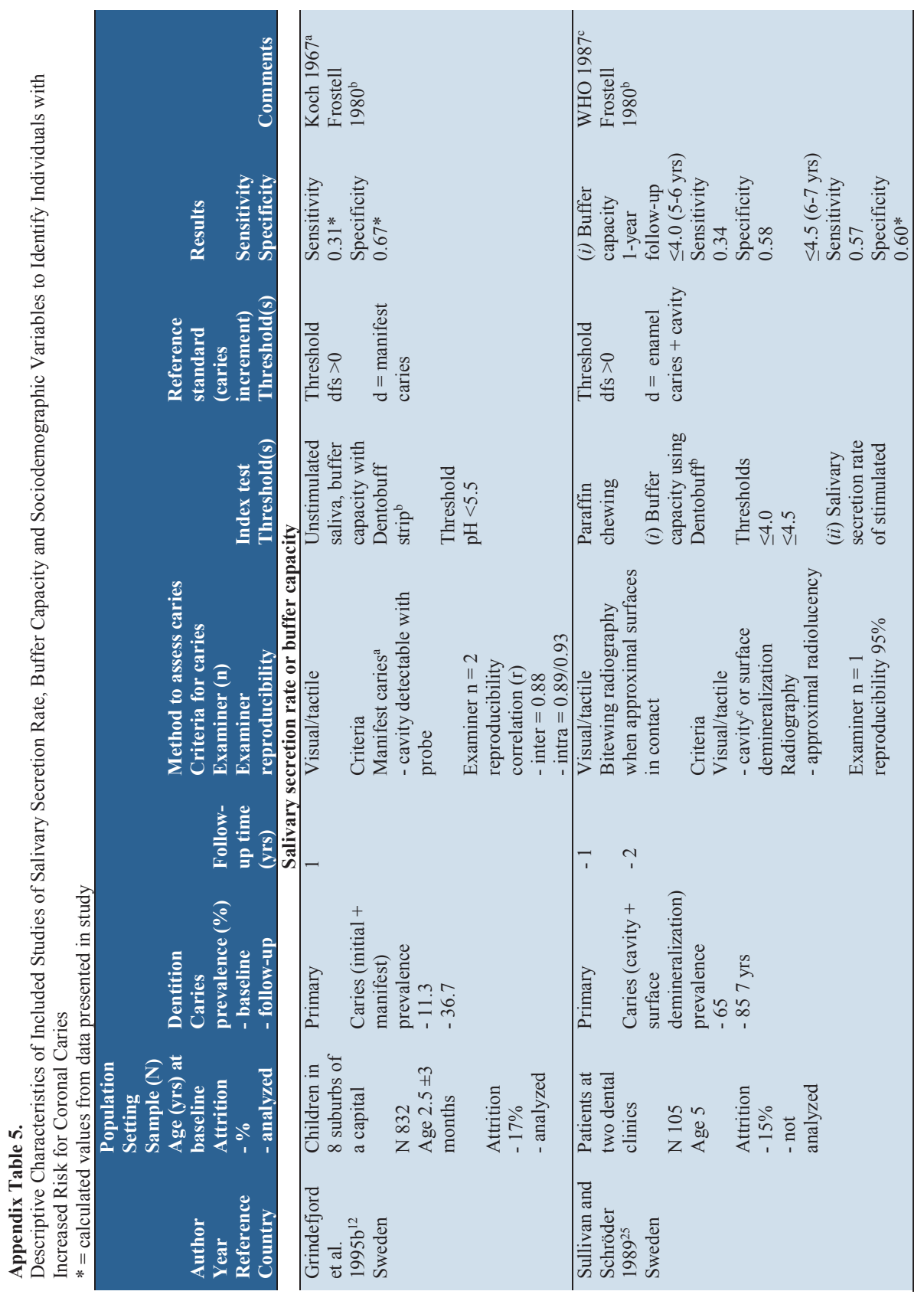




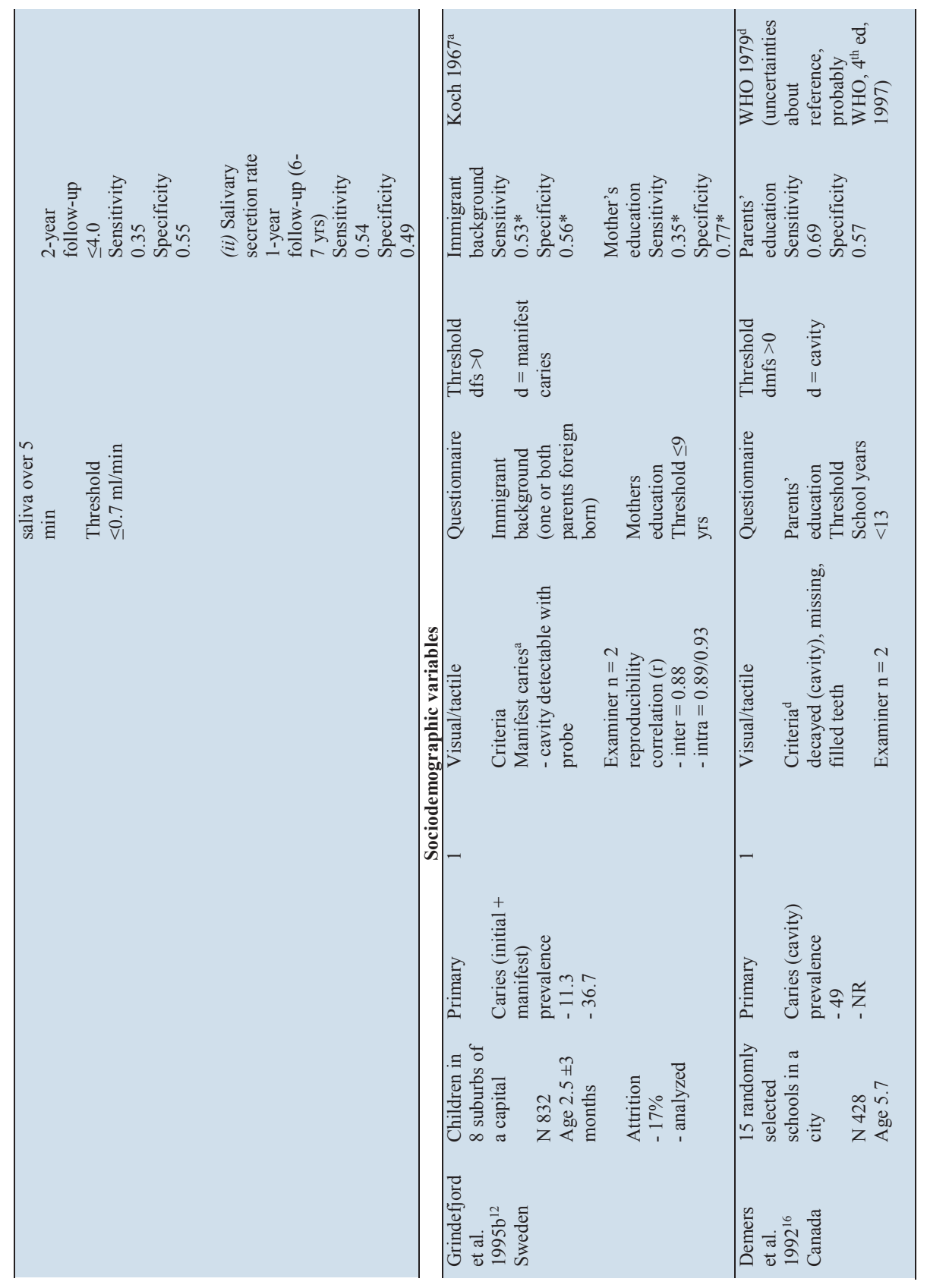




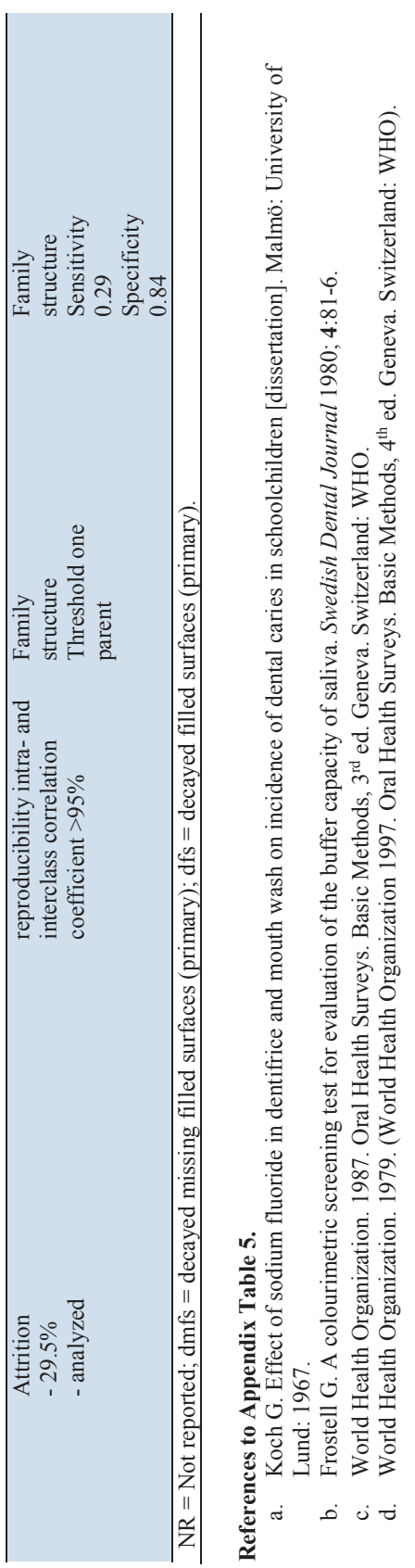




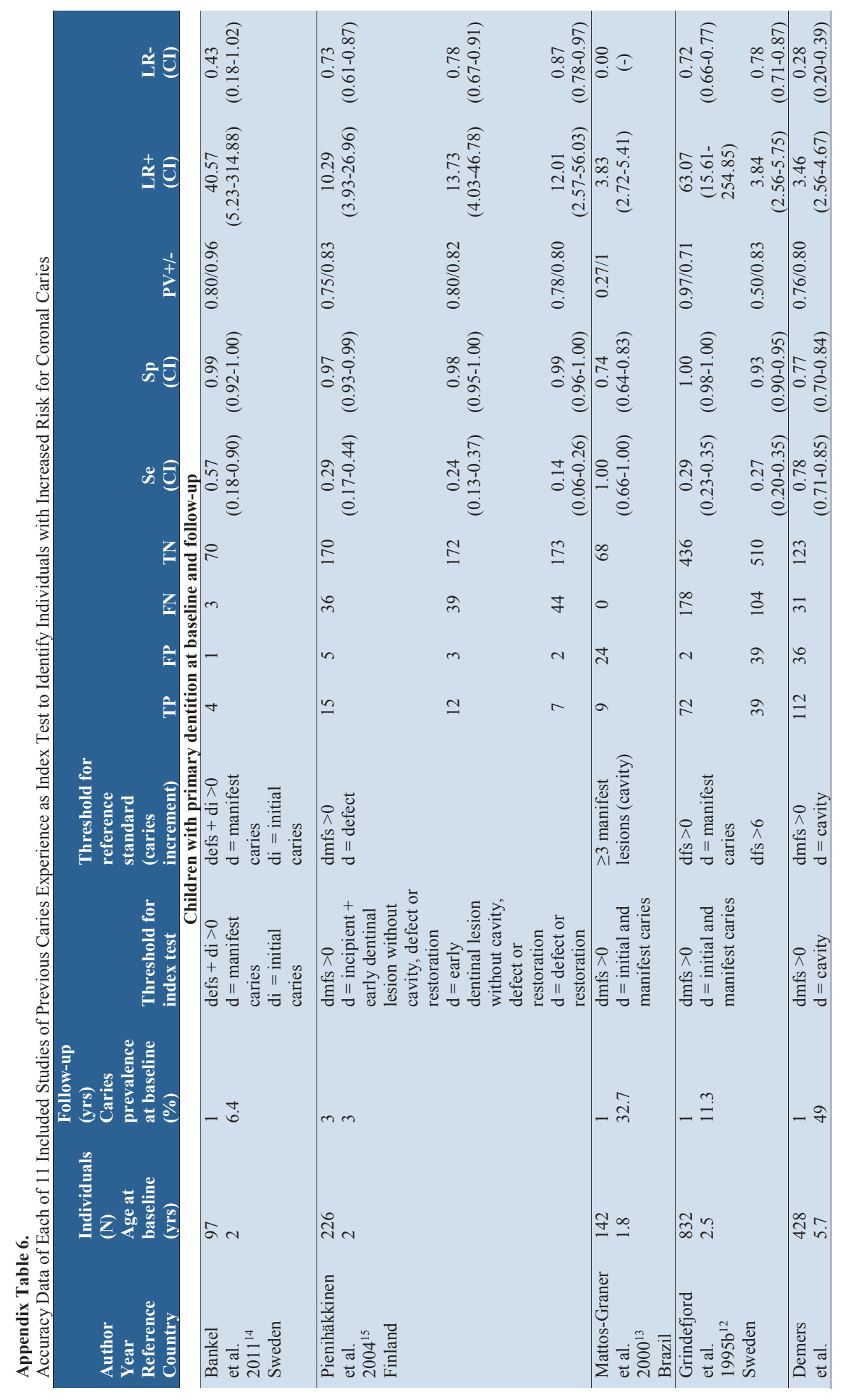




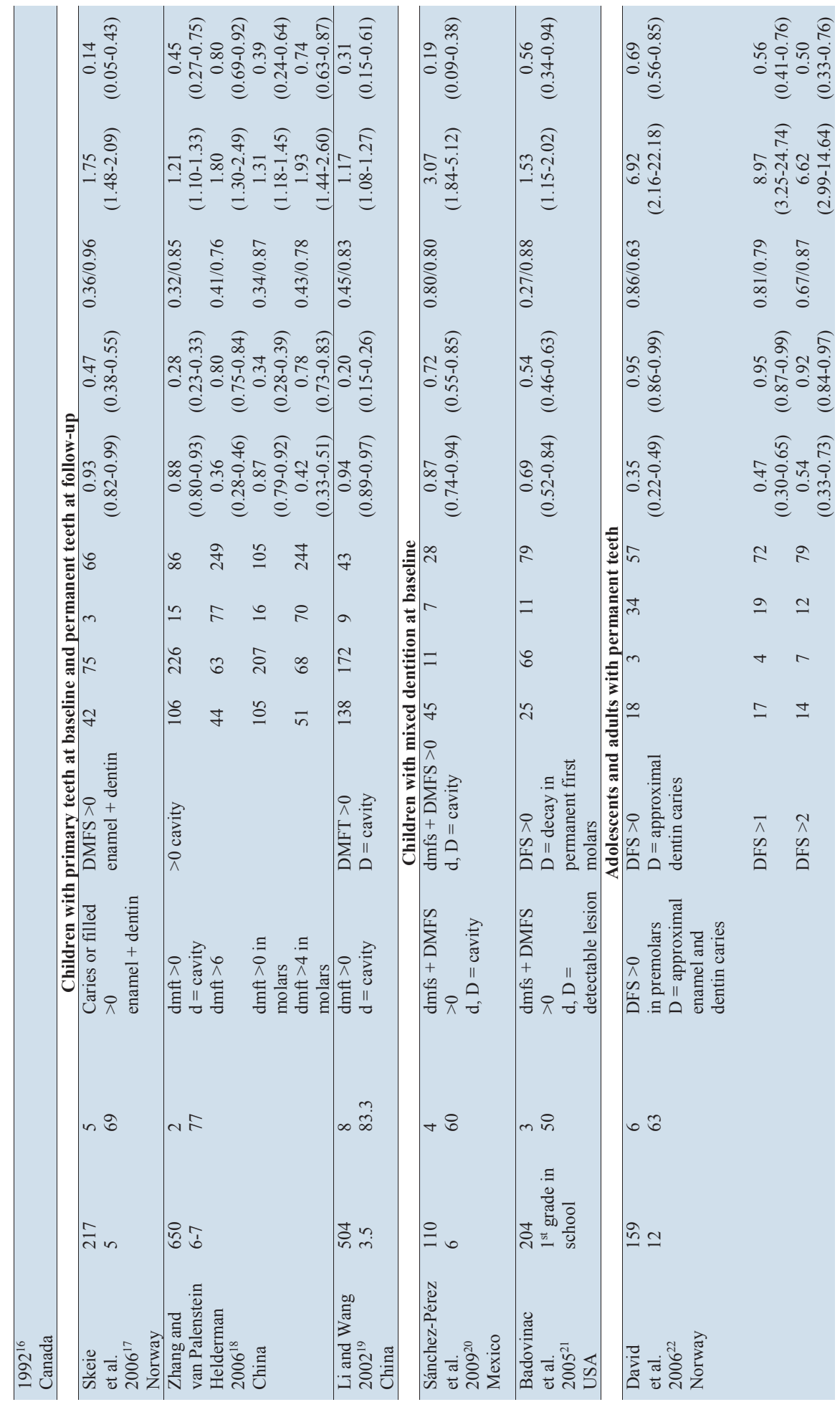




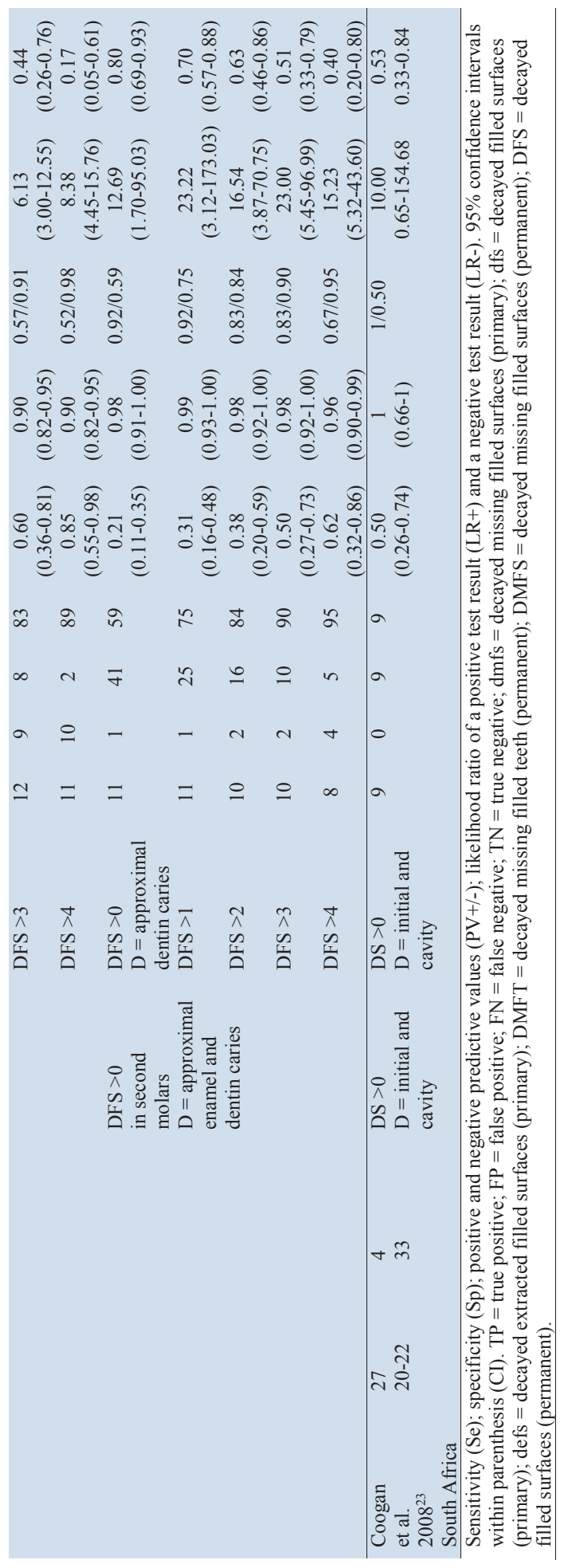




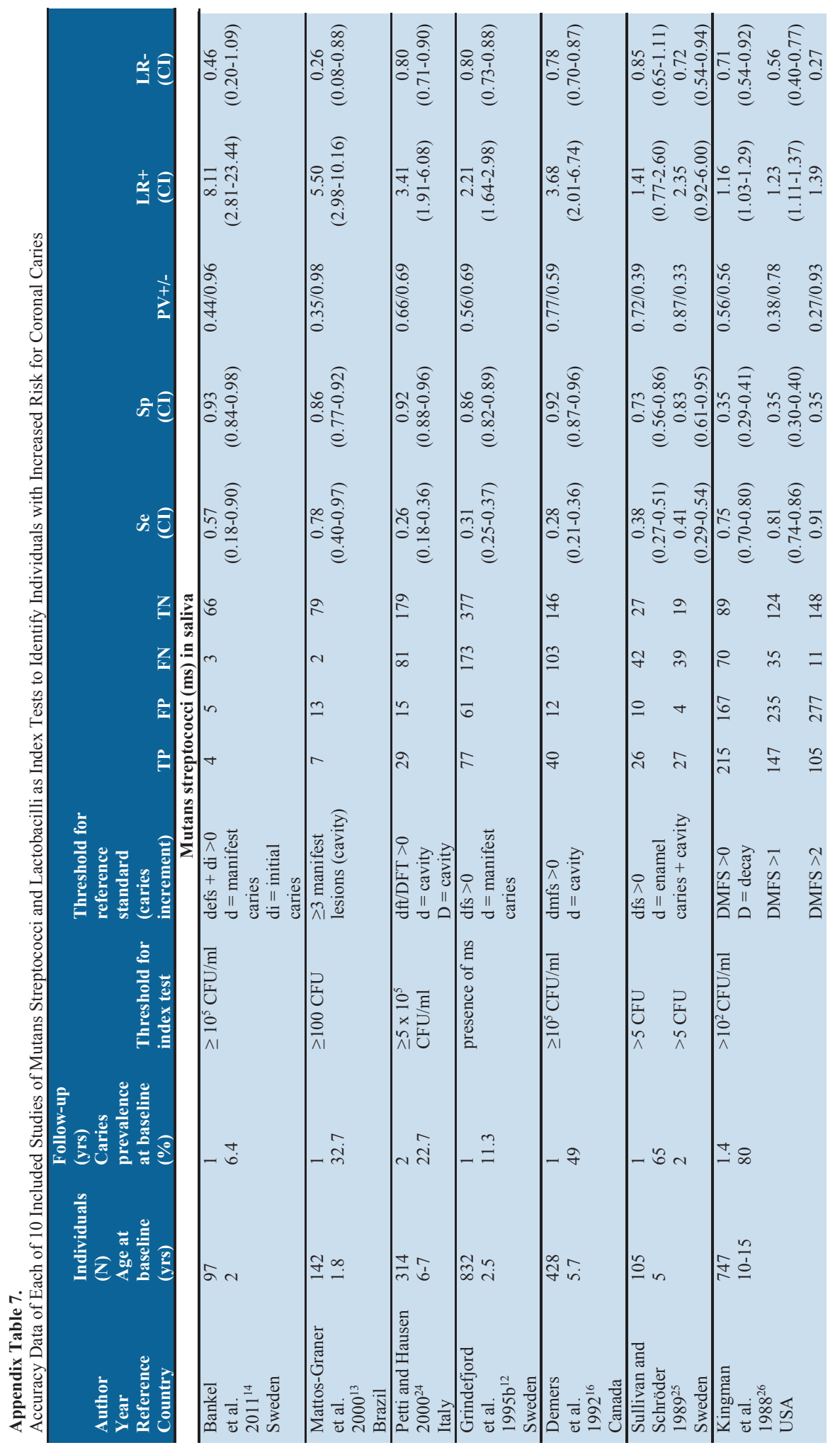




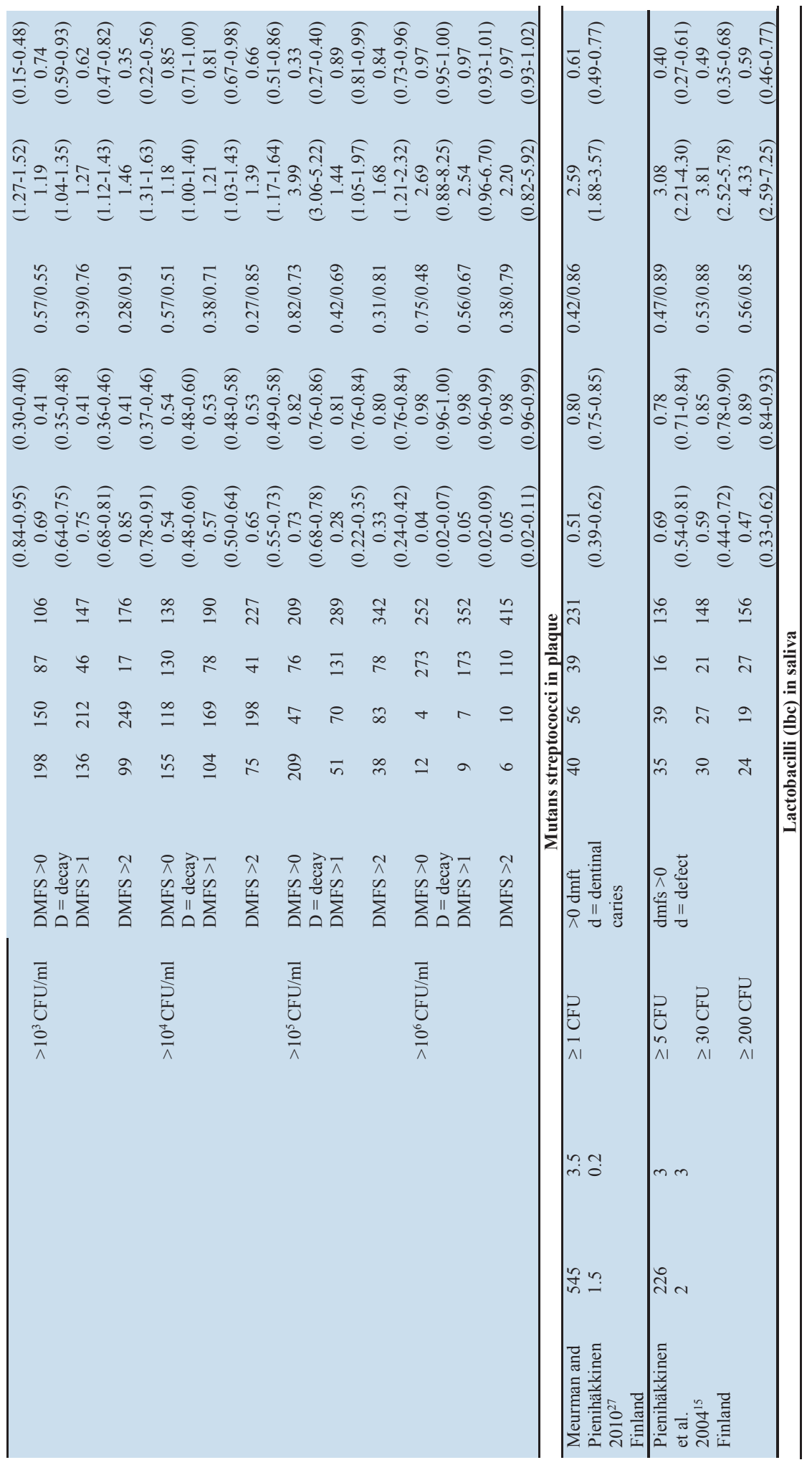




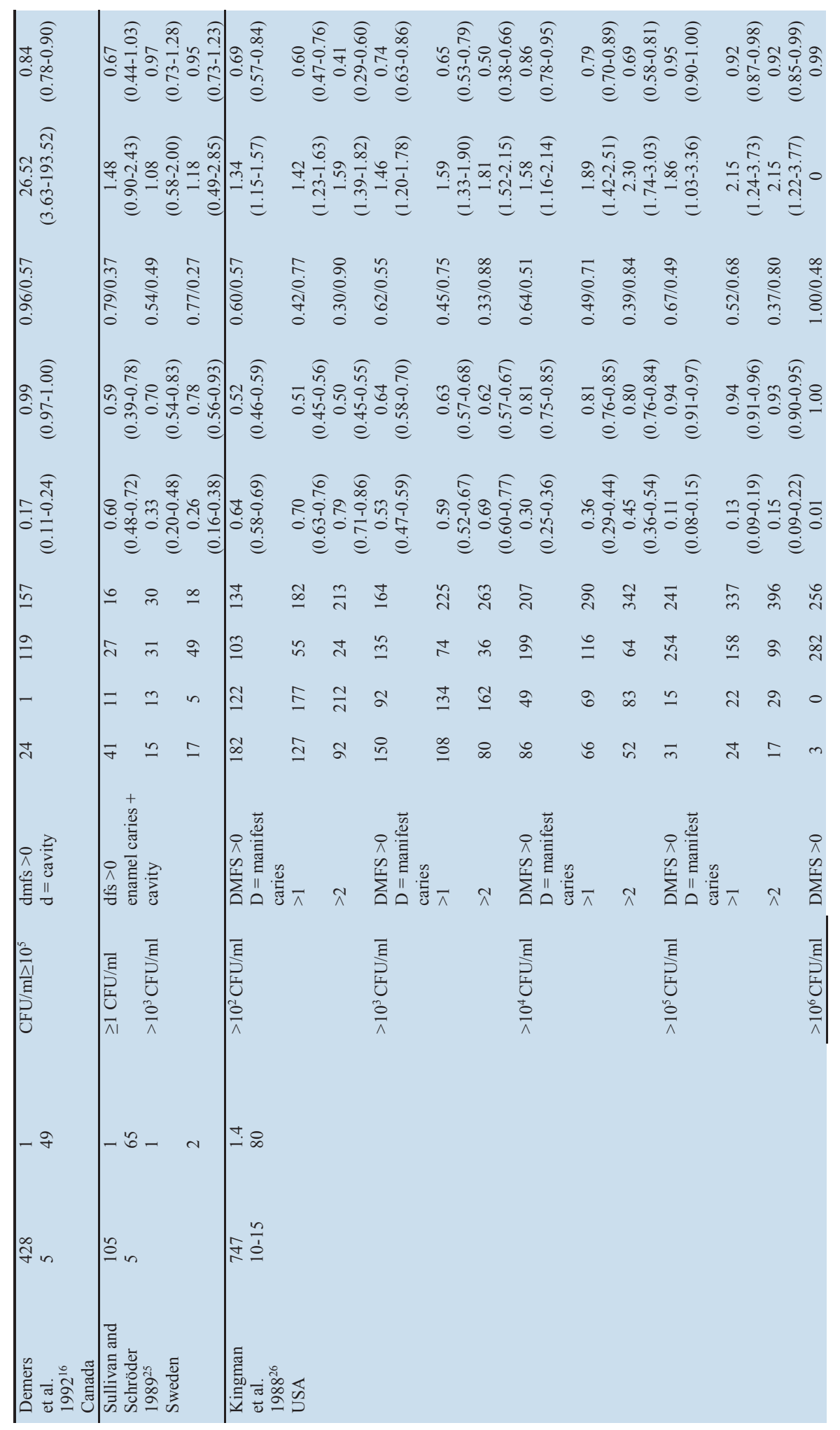




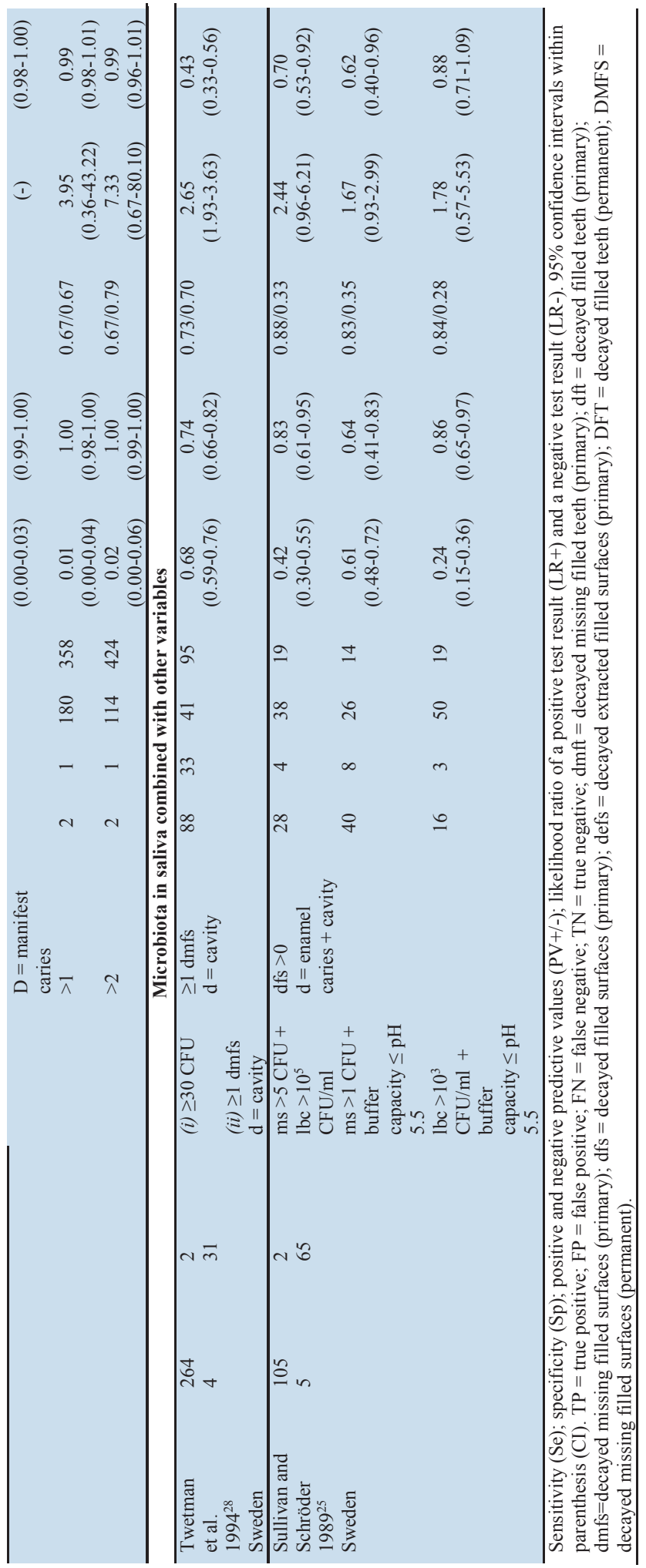




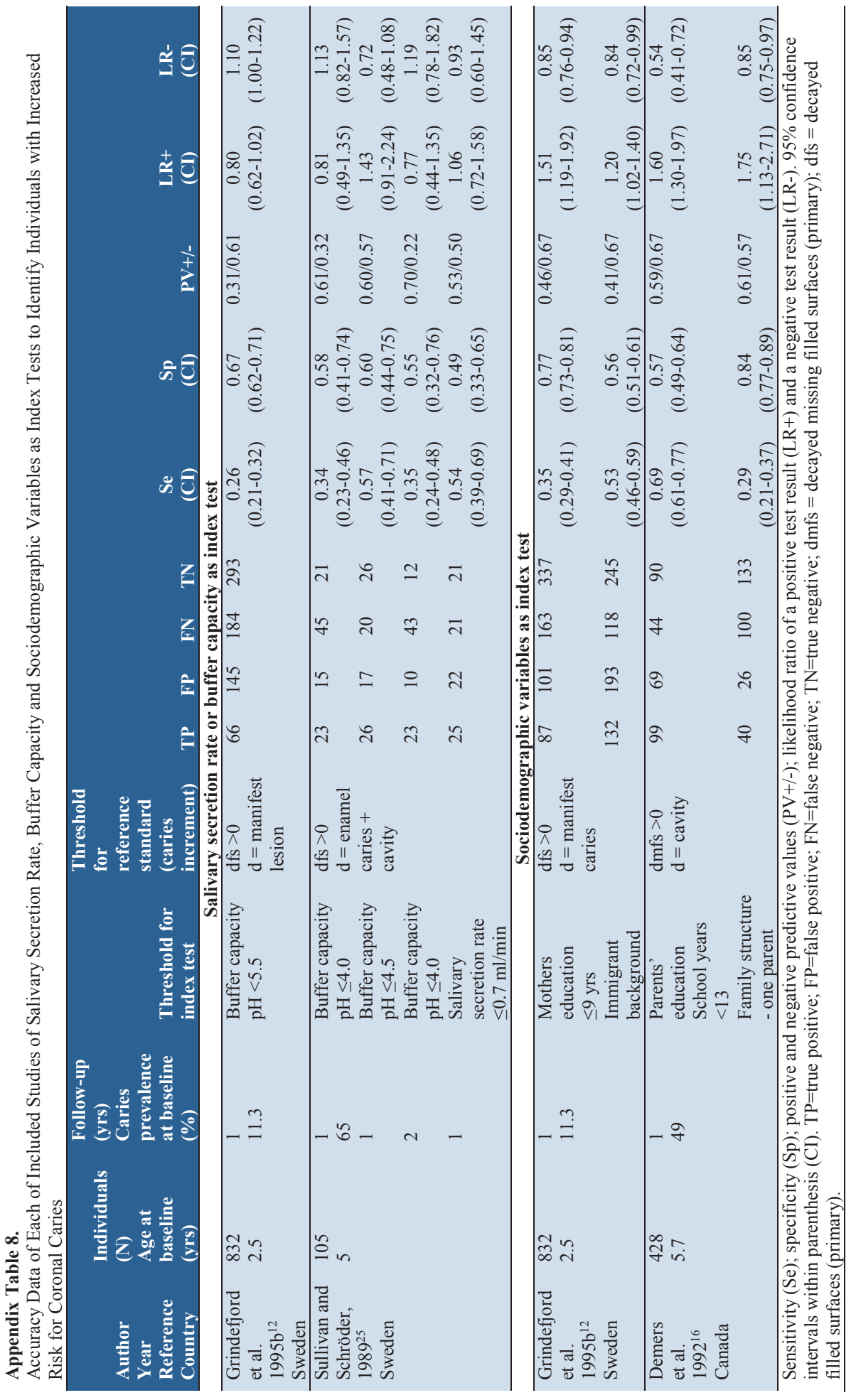



II 



\title{
RESEARCH ARTICLE \\ A novel classification system for assessment of approximal caries lesion progression in bitewing radiographs
}

\author{
${ }^{1}$ Anna Senneby, ${ }^{2}$ Margareta Elfvin, ${ }^{3}$ Christina Stebring-Franzon and ${ }^{1}$ Madeleine Rohlin \\ ${ }^{1}$ Faculty of Odontology, Malmö University, Malmö, Sweden; ${ }^{2}$ Färjestaden General Dental Clinic, Public Dental Health Service, \\ Sweden; ${ }^{3}$ Alvesta General Dental Clinic, Public Dental Health Service, Sweden
}

\begin{abstract}
Objectives: To design and pilot a novel classification system for the assessment of caries lesion progression in bitewing radiography and to report rater agreement of the system.

Methods: A classification system with drawings and text was designed to assess caries lesion progression. Guidelines for Reporting Reliability and Agreement Studies were used to study and report rater agreement. Pairs of posterior bitewing radiographs (baseline and 1-year follow-up) with different status concerning caries lesion progression were selected from files from public dental health clinics. 10 raters, 5 general dental practitioners and 5 specialists in oral and maxillofacial radiology were asked to assess the radiographs with the aid of the classification system. Seven raters repeated their assessments. Rater agreement was expressed as percentage of agreement and kappa. Results: Kappa for the interrater agreement of 10 raters assessing progression was 0.61 , indicating substantial agreement. Agreement was moderate for progression in the outer half of the dentine (kappa 0.55) and within enamel (kappa 0.44). Pairwise interrater agreement varied (range 69-92\%; kappa $0.42-0.84$ ). For about half of the pairs of raters, kappa was substantial $(\geq 0.61)$. Intrarater agreement assessing progression was substantial (kappa 0.66-0.82).

Conclusions: We demonstrated the applicability of the proposed classification system on caries lesion progression with respect to rater agreement. This system can provide a common framework for clinical decision-making on caries interventional methods and patient visiting intervals. Scientifically, this system allows for a comparative analysis of different methods of prevention and treatment of caries as well as of different caries risk assessment methods. Dentomaxillofacial Radiology (2016) 45, 20160039. doi: 10.1259/dmfr.20160039
\end{abstract}

Cite this article as: Senneby A, Elfvin M, Stebring-Franzon C, Rohlin M. A novel classification system for assessment of approximal caries lesion progression in bitewing radiographs. Dentomaxillofac Radiol 2016; 45: 20160039.

Keywords: dental caries; disease progression; visual perception; observer variation; radiography bitewing; classification

\section{Introduction}

Alongside visual/tactile examination, radiography is well established as a diagnostic tool to detect caries. According to a recent meta-analysis, the sensitivity for detecting caries

Correspondence to: Anna Senneby. E-mail: anna.senneby@mah.se

The study was funded by a grant received from Malmö University "Grant for research students" and from the Borrow Foundation. The Borrow Foundation had no role in the study design, data collection, analysis and interpretation of data, in the writing of the report or in the decision to submit the article for publication.

Received 24 January 2016; revised 23 March 2016; accepted 31 March 2016 in clinical and in vitro studies is 0.24 and 0.42 , respectively, for approximal lesions and corresponding specificity is, 0.97 and $0.89 .{ }^{1}$ Based on the results of clinical studies, the mean sensitivity for detecting dentinal lesions in radiographs is 0.36 and based on in vitro studies, it is 0.45 . The mean specificity is 0.94 and 0.95 , respectively. ${ }^{1}$ Reported rater agreement varies among radiographic studies of caries detection; ${ }^{2-5}$ this may be owing to differences in underlying sample characteristics, such as lesion depth, dentition, surface location and caries prevalence, and to 
methodological heterogeneity, such as the number of surfaces, number of raters and scoring categories.

In general dental healthcare, clinicians often have access to a series of radiographs of their patients from different years. The decision on intervention of approximal caries can then be based on not only the clinical examination and the detection of caries lesions and lesion depth in the radiographs but also the caries lesion progression rate. In addition, when evaluating different types of interventions, accurate assessment of caries lesion progression is important in everyday clinical practice and in the context of scientific studies. Assessment of caries lesion progression is also crucial in studies of caries risk assessment, as caries increment, i.e. the development of new or more severe disease, comprises the reference standard of choice. A recent systematic review of methods for caries risk assessment demonstrated a lack of clear definition of diagnostic criteria and data on rater variability for the assessment of caries lesion progression. This resulted in a methodological heterogeneity among the included studies. ${ }^{6}$ Although caries lesion progression is assessed radiographically daily in clinical practice and in several scientific studies, the level of agreement among raters is largely unknown. To our knowledge, there is only one previous publication on the reproducibility of radiographic assessment of caries lesion progression. ${ }^{7}$

In view of the importance of the radiographic assessment of caries lesion progression, an established classification system for this assessment could prove valuable. It would have the potential to facilitate clear communication among clinicians and researchers. Preferably, a novel classification system should be evaluated concerning its diagnostic accuracy and correspond to changes in disease status. However, accuracy of radiographic assessment of caries lesion progression in terms of sensitivity and specificity is difficult to achieve owing to difficulties in obtaining a reference standard. Wenzel et $\mathrm{al}^{8}$ stated, "when no valid expression of the true state of the disease can be obtained a study of precision ... is appropriate". Swets and Pickett ${ }^{9}$ also highlighted that observer performance could be considered as a measure of the extent to which the inaccuracy of a system is the result of decision-making errors. Based on the reported variation among dentists in the detection and depth estimation of caries lesions, it can be expected that this variation may also exist for the assessment of caries lesion progression.

Hence, the objective of this study was to design a classification system for the assessment of approximal caries lesion progression in bitewing radiography and to pilot its applicability in terms of rater agreement among a group of general dental practitioners (GDPs) and oral and maxillofacial radiologists.

\section{Material and methods}

In order to achieve an approach that enables the readership to assess potential bias, this study implemented the guidelines proposed in the Guidelines for Reporting
Reliability and Agreement Studies (GRRAS). ${ }^{10} \mathrm{We}$ used a GRRAS checklist with modifications (Supplementary Table A). ${ }^{11}$

\section{Sample of adolescents}

Adolescents, aged 12 years, from two public dental health clinics (Kronoberg and Blekinge County, Sweden) were consecutively enrolled when visiting the clinic for regular check-up appointments. The enrolment took place during a recruitment period for a prospective clinical trial in 2012-2014. The adolescents were examined by visual and tactile examination using a mirror and probe. Then, one bitewing radiograph was exposed in each side of the mouth. The examinations were repeated yearly. Ethical approval was given by the Regional Ethical Review Board, Lund, Sweden (diary number: OD62-2012/122).

\section{Radiographic equipment and quality control}

Dental X-ray units (Planmeca Intra, Helsinki, Finland) operating at $8 \mathrm{~mA}$ and $63 \mathrm{kV}$ with a rectangular positioning device and a focus-to-skin distance of $0.35 \mathrm{~m}$ were used. The X-ray units were equipped with electronic timers. Exposure time was set to $0.25 \mathrm{~s}$, but in applicable cases, this was adjusted to $0.32 \mathrm{~s}$. The two imaging plate systems used were Soredex Digora ${ }^{\mathbb{R}}$ (Soredex, Finland) with a $40 \times 30 \mathrm{~mm}^{2}$ effective area and Dürr VistaScan ${ }^{\odot}$ (Dürr Dental AG, Germany) with a $28 \times 36 \mathrm{~mm}^{2}$ effective area. The imaging plates offered a pixel size of $39 \times 39$ and $45 \times 45 \mu^{2}$, respectively. The imaging plates were enveloped in a plastic protective sheet and fixed to a Kwik-Bite ${ }^{\mathbb{B}}$ film holder (Kerr Corporation, USA).

Prior to the radiographical examination, the first author (AS) checked the radiological equipment for the following parameters: tube voltage, exposure time (reproducibility and linearity), low contrast and spatial resolution. The doses of radiation (microgray) for different exposure times were recorded. In addition, dose ratios (milligray per second) were recorded for applicable exposure times $(0.2,0.25$ and $0.32 \mathrm{~s})$. Regarding tube voltage, thresholds for kilovoltage were adjusted $(60,63,66$ and $70 \mathrm{kV})$ and the actual tube voltage was recorded with RaySafe ThinX Intra equipment (Unfors RaySafe, Fluke Biomedical, WA). When exposure times were registered, a scheme of five repetitions per time setting based on the clinically applicable exposure times was adopted for the reproducibility control. Further, a series ranging from 0.01 to $0.5 \mathrm{~s}$ of exposure time was used for the linearity control. Doses of radiation were measured throughout and dose ratios calculated on the obtained values. Spatial resolution was measured by exposing a spatial resolution phantom three times with different exposure times intended to produce optimal visualization of the object in the computer software used. Two plexiglass plates served as a phantom meant to represent the soft tissues of patients, thus being placed in between the spatial resolution phantom and the imaging plate. A value, visible 
lines per millimetre, was obtained by reviewing the radiographic image (expressed as a number of 1-10). In order to obtain the best possible image of low contrast reproducibility, a plexiglass rectangle with depressions of different depths was used.

The images were saved in digital software programs: Arion (Pro Curis AB, Sweden) or Effica Viewer (Tieto Healthcare and Software AB, Finland). All images were exported as digital imaging and communication in medicine files ${ }^{12}$ to portable storage media from the digital viewing software programs. The images were then imported to Romexis ${ }^{\mathbb{R}}$ software (Planmeca, Finland) and were anonymized.

\section{Classification system and selection of bitewing} radiographs and approximal surfaces

A classification system with drawings was designed to visualize disease status in terms of no caries lesion progression or caries lesion progression (Figure 1). Parallel to the drawings, text and indices were included to clarify different status of the approximal surfaces.

Two of the authors (AS, MR) selected the bitewing radiographs of approximal surfaces to be included in the study. During the selection process, image quality regarding contrast, sharpness, brightness and projection as well as lesion progression were considered. Images in each patient were paired: one image from baseline and one image from the 1-year follow-up. Radiographs were selected to represent five surfaces for all categories presented in Figure 1, except for surfaces with caries lesion in the inner half of the dentine (Categories 4,8 , 11,13 and 14), which were not represented in the sample and not assessed by the raters. 20 sound surfaces without progression were included. Altogether, 65 approximal surfaces with and without caries lesion progression were included, 39 surfaces in first molars and 26 surfaces in premolars from any quadrant with 1 surface reoccurring in the sample. All selected surfaces were non-restored, five surfaces were adjacent to a restored occlusal surface and one surface was adjacent to a restored approximal surface. Fillings in other surfaces were composite alike in radiolucency. Moreover, of the 65 surfaces with 2 radiographs of each (resulting in 130 images), 17 surfaces overlapped with the neighbouring surface, but the overlap did not extend beyond the outer half of the enamel.

\section{Raters and ratings}

10 raters, 5 GDPs and 5 specialists in oral and maxillofacial radiology were asked to assess the approximal surfaces. The GDPs worked at five different general dental clinics in three different public dental health counties. The specialists were currently employed at or had recently been employed at the Department of Oral

\section{NO PROGRESSION}

0<smiles>ClCCCC1CC2CC3CC(C2)CC1C3</smiles>

1

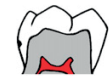

Outer enamel

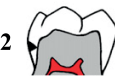

Inner enamel

3

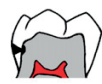

Outer dentine

4

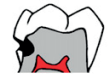

Inner dentine

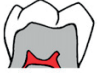

Sound

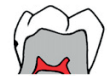

Outer enamel

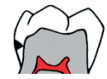

Inner enamel

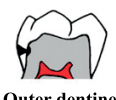

Outer dentine

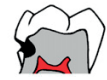

Inner dentin
5

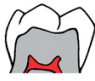

Sound

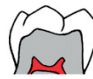

Sound

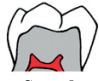

Sound

8

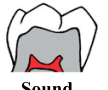

Sound

\section{PROGRESSION}

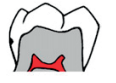

Outer enamel

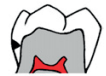

Inner enamel

10
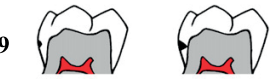

Outer enamel Inner enamel

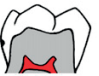

Outer ename

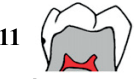

Outer enamel

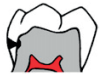

Outer dentine
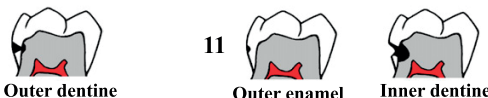

Inner dentin

12
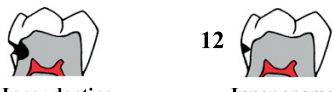

Inner enamel

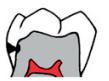

13

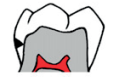

Outer dentine

Inner enamel

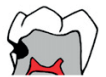

14

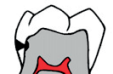

Outer dentine Inner dentin

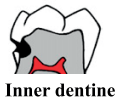

Figure 1 Classification system for the radiographic assessment of caries lesion progression in approximal surfaces. Caries lesions were categorized as follows: "Outer enamel" represents lesions in the outer half of enamel; "Inner enamel" represents lesions in the inner half of enamel; "Outer dentine" represents lesions in the outer half of dentine; "Inner dentine" represents lesions in the inner half of dentine. 
and Maxillofacial Radiology, Malmö University. The selection of raters was made in such a way as to ensure a diverse level of professional experience. The professional experience of the GDPs ranged between 1 and 36 years (mean 13.8 years). The experience of the specialists in their field of speciality ranged between 1 and 33 years (mean 19.2 years).

All assessments took place in one viewing session at the Department of Oral and Maxillofacial Radiology, Malmö University, using Romexis software and a computer (HP Compaq Elite 8100 SFF; HewlettPackard, CA). Before the assessments, contrast and brightness of the monitor were visually adjusted with test images TG18-QC and TG18-CT from the American Association of Physicists in Medicine Task Group 18 Assessment of Display Performance for Medical Imaging Systems. ${ }^{13}$

Images were viewed on an 18.1-inch greyscale liquid crystal display monitor (Medical Flat Greyscale Display, 1318, Barco, Belgium) set at a screen resolution of $1280 \times 1024$ pixels, 8-bit colour depth, with an original luminance of $400 \mathrm{~cd} \mathrm{~m}^{-2}$ and 0.2805 pixel pitch. The room had dimmed light (ambient light below 20 lux), and the viewing distance to the screen was $30-40 \mathrm{~cm}$. No image enhancement or altering of image characteristics was allowed. The images were displayed side by side. The software, which was applied, used bilinear interpolation for scaling of images on screen. During the viewing sessions, which were performed independently by each observer, one of the authors (AS) was available to answer questions and to fill out the protocols. For each included approximal surface, the images from baseline were read simultaneously with those from the follow-up. Prior to the ratings, the raters were allowed to view pairs of bitewing images serving as examples of upcoming assessments. The raters were informed that the bitewing radiographs were from adolescents and that the pair of radiographs represented one from baseline and one from a follow-up examination. They did not receive any additional clinical information. The raters were aware that their ratings were to be compared with those of other raters.

The raters independently assessed the surfaces according to the classification system (Figure 1), which was available to the raters during their assessments. The raters were told which tooth and surface to assess. Seven raters (three GDPs and four specialists) reassessed all surfaces in the same order and same setting after 2 4 weeks. During the second viewing, raters were blinded towards their results from their previous assessments.

\section{Statistical analysis}

Statistical analysis was performed with the aid of a statistician at Malmö University. The analysis was performed using STATA 10 (StataCorp, LP, TX). Kappa values with $95 \%$ confidence intervals (CI) were generated for intrarater and interrater agreement. For the pairwise interrater and intrarater agreement, the percentage of agreement was also calculated. Interrater agreement calculations were based on the first reading. Kappa values, varying from -1 to 1 , were categorized according to the scale suggested by Landis and Koch. ${ }^{14}$ According to this scale, values $<0$ are considered as poor agreement, $0.00-0.20$ slight, $0.21-0.40$ fair, $0.41-0.60$ moderate, $0.61-0.80$ substantial and values $>0.81$ as almost perfect agreement.

\section{Results}

All selected 65 surfaces and all rater assessments were included for final analysis. Substantial interrater agreement was achieved for 10 raters for the assessment of caries lesion progression. Intrarater agreement was generally higher than interrater agreement.

\section{Interrater agreement}

Agreement in rating progression was substantial among 10 raters (kappa 0.61 ), being 0.57 among 5 GDPs and 0.65 among 5 specialists (Table 1). Agreement of 10 raters was moderate for the assessment of progression in the outer half of the dentine (kappa 0.55) and within the enamel (kappa 0.44) (Table 1). For surfaces assessed as sound without progression, agreement was substantial (kappa 0.78).

Pairwise interrater agreement regarding progression ranged between $69 \%$ and $92 \%$ with corresponding kappa values from 0.42 to 0.84 (Table 2). About half of the kappa values indicated at least substantial agreement $(\geq 0.61)$. Two raters (GDP 2 and oral radiologist 4) presented moderate pairwise agreement with all other raters (Table 2)

Table 1 Interrater agreement expressed as kappa with confidence intervals (CI) for the assessment of caries lesion progression of approximal surfaces $(n=65)$ in bitewing radiography of adolescents

\begin{tabular}{|c|c|c|c|}
\hline \multirow[b]{2}{*}{ Raters } & \multicolumn{3}{|l|}{ Agreement } \\
\hline & $\begin{array}{l}\text { All surfaces assessed to present } \\
\text { progression }(5,6,7,9,10,12)^{a} \\
\text { Kappa }(C I)\end{array}$ & $\begin{array}{l}\text { Surfaces assessed to present } \\
\text { progression within the enamel } \\
(5,6,9)^{a} \\
\text { Kappa }(C I)\end{array}$ & $\begin{array}{l}\text { Surfaces assessed to present } \\
\text { progression in the outer half of the } \\
\text { dentine }(7,10,12)^{a} \\
\text { Kappa }(C I)\end{array}$ \\
\hline All raters $(n=10)$ & $0.61(0.54-0.70)$ & $0.44(0.34-0.54)$ & $0.55(0.37-0.73)$ \\
\hline $\begin{array}{l}\text { General dental practitioners } \\
(n=5)\end{array}$ & $0.57(0.45-0.69)$ & $0.53(0.38-0.67)$ & $0.61(0.36-0.80)$ \\
\hline Oral radiologists $(n=5)$ & $0.65(0.52-0.77)$ & $0.43(0.30-0.57)$ & $0.64(0.49-0.79)$ \\
\hline
\end{tabular}

${ }^{a}$ Numbers refer to categories presented in Figure 1 . 
Intrarater agreement

Intrarater agreement was above $80 \%$ for the assessment of caries progression (Table 3). Expressed as kappa, the agreement was substantial for all observers (mean 0.76; range 0.66-0.82). Agreement varied among raters for the assessment of progression in the outer half of the dentine (86-97\%; kappa $0.52-0.90$ ) (Table 3) and for progression within the enamel (82-91\%; kappa 0.52-0.73) (Table 3). For surfaces assessed as sound without progression, agreement ranged between $94 \%$ and $100 \%$ (kappa $0.86-1.00$ ). No rater consistently presented the highest or lowest intrarater agreement, but the CI were somewhat wider for some raters.

\section{Discussion}

The present study proposes a novel classification system for the assessment of caries lesion progression in bitewing radiography. The results on rater agreement demonstrate that it is possible to achieve substantial agreement between several raters as well as within raters.

During the last decades, numerous systems have been proposed for the radiographic detection of approximal caries lesions. These systems are based on the interpretation of one radiograph at one point in time. ${ }^{15-20}$ Since a single radiograph with radiolucency should not be misinterpreted as indicating active disease, ${ }^{21}$ an approach with a series of images may be more beneficial to clinical decision-making. Even though it has long been called for in the scientific literature, ${ }^{16,22,23}$ to our knowledge, no classification system based on the radiographic assessment of change of approximal surfaces over time has ever been presented. The classification system presented in this study builds on existing systems of caries lesion detection such as the International Caries Classification and Management System (ICCMS ${ }^{\mathrm{TM}}$ ), ${ }^{24}$ which in turn is based on the widely used International Caries Detection and Assessment System (ICDAS $\left.{ }^{\mathrm{TM}}\right) .{ }^{20}$ It might be beneficial to follow these criteria, as the staging of caries lesion is already known to clinicians and researchers. However, the fundamental difference is that the classification system presented in this study is designed for the comparison of two images performed with a time interval, in order to detect changes of the dental tissues. The drawings in the classification system are designed to show the caries lesion depths and lesion progression. To facilitate the interpretation of the drawings, the caries lesions within the enamel are presented as triangles. Other appearances of enamel caries lesions, such as a band or a dot, were also represented in the sample. Minor differences of contrast, e.g. in intraoral radiography of caries lesion progression, are more evident when comparing a series of images. This is particularly the case when the small changes in density within the same object are imaged against a homogeneous background. ${ }^{2}$ 
Table 3 Intrarater agreement expressed as percentage of agreement (\%) and kappa with confidence interval (CI) for the assessment of caries lesion progression of approximal surfaces $(n=65)$ in bitewing radiography of adolescents

\begin{tabular}{|c|c|c|c|c|c|c|c|}
\hline \multirow[b]{3}{*}{ Raters } & \multicolumn{7}{|c|}{ Agreement } \\
\hline & & \multicolumn{2}{|c|}{$\begin{array}{l}\text { All surfaces assessed to present } \\
\text { progression }(5,6,7,9,10,12)^{a}\end{array}$} & \multicolumn{2}{|c|}{$\begin{array}{l}\text { Surfaces assessed to present } \\
\text { progression within enamel }(5,6,9)^{a}\end{array}$} & \multicolumn{2}{|c|}{$\begin{array}{l}\text { Surfaces assessed to present progression in } \\
\text { outer half of dentine }(7,10,12)^{a}\end{array}$} \\
\hline & & $\%$ & Kappa $(C I)$ & $\%$ & Kappa $(C I)$ & $\%$ & Kappa $(C I)$ \\
\hline General dental & 18 & 89 & $0.75(0.58-0.92)$ & 89 & $0.67(0.45-0.90)$ & 91 & $0.52(0.19-0.86)$ \\
\hline \multirow{2}{*}{ practitioners } & 28 & 88 & $0.74(0.57-0.91)$ & 88 & $0.70(0.52-0.89)$ & 97 & $0.82(0.57-1.00)$ \\
\hline & 38 & 89 & $0.78(0.62-0.93)$ & 83 & $0.55(0.32-0.79)$ & 94 & $0.76(0.54-0.98)$ \\
\hline \multirow[t]{4}{*}{ Oral radiologists } & 18 & 83 & $0.66(0.47-0.84)$ & 82 & $0.52(0.29-0.76)$ & 92 & $0.74(0.52-0.96)$ \\
\hline & 29 & 91 & $0.82(0.68-0.96)$ & 83 & $0.57(0.34-0.80)$ & 86 & $0.60(0.37-0.83)$ \\
\hline & 38 & 89 & $0.78(0.63-0.93)$ & 89 & $0.71(0.51-0.91)$ & 88 & $0.56(0.29-0.83)$ \\
\hline & 49 & 91 & $0.81(0.67-0.95)$ & 91 & $0.73(0.53-0.93)$ & 97 & $0.90(0.76-1.00)$ \\
\hline
\end{tabular}

${ }^{a}$ Numbers refer to categories presented in Figure 1.

Psychologically, the comparison of two images side by side in order to detect signs of tissue changes over time involves a different mindset than the study of a single image. In the diagnostic process, raters make a series of inferences derived from existing observations, one example being historical data. ${ }^{26}$ In our context, historical data refer to the possibility of comparing two images exposed at different times. With access to serial radiographs over time, any small change of the hard tissues should be assessed. With respect to dental caries, not only lesion progression but also lesion regression, i.e. surface remineralization of enamel lesions, is important to assess, in particular when evaluating the efficacy of different non-invasive methods. Caries lesion regression was not included in the present classification system.

Research on observer performance views diagnosis as both a perceptual and a cognitive phenomenon that consists of learning the cues. ${ }^{27,28}$ Various aids have been applied to present cues characteristic of different categories, in order to decrease errors in perception and diagnostic reasoning. The present classification system designed and then piloted in our study has the advantage of presenting cues both as verbal descriptions and as visual patterns. There is ample confirmation from studies in visual diagnosis, primarily in radiology, that when data are elicited as discrete features, there is a large perceptual component that rapidly recognizes patterns rather than a cognitive component that seeks data for further discrimination. ${ }^{29}$ In line with this concept of pattern recognition, the present classification system was designed to describe caries lesion progression with illustrations. The side-by-side illustrations are drawn to resemble radiographic images with features illustrating approximal surfaces with and without caries lesion progression. Seeing features within an appropriate context induces familiarity effects and will underpin categorization. ${ }^{26}$

As different raters may have different visual concepts and prior experience, a study of rater agreement anticipates a design with several raters, in particular when the number of surfaces is modest. ${ }^{30}$ In this study, low caries prevalence and few dentinal lesions limited the number of surfaces. Including more than six or seven raters has little consequence on the results when a reasonably large sample is examined. ${ }^{9}$ Raters included in this study represented different professional experiences working in different institutions as well as different time lengths of expertise (long, intermediate and short time of experience). Yet, interrater agreement of 10 raters for caries lesion progression indicated substantial agreement (kappa 0.61), which is comparable with the results for 7 raters (kappa 0.70) in the study of caries lesion development by Wenzel et al. $^{7}$ Pairwise interrater agreement varied, but substantial agreement was reached for many pairs of raters. The average intrarater agreement was 0.76 , identical to that presented by Wenzel et $\mathrm{al}^{7}$ for the assessment of caries lesion behaviour. Although difficult to compare, these results indicate higher interrater agreement than for caries detection by bitewing radiography reported in studies of high and medium quality.

The magnitude of agreement is not solely a statistical decision, but also depends on the purpose and consequences of the test results. Even if the guidelines suggested by Landis and $\mathrm{Koch}^{14}$ are widely adopted when reporting rater agreement, there have been problems when interpreting the kappa coefficients. Interpretation against arbitrary standards does not distinguish between different applications of the guidelines; for example, the reliability may be acceptable for use in a research setting but inadequate for clinical decision-making on individual patients. Clinically, there are different courses of action available in caries lesion progression: (i) non-invasive (early) intervention, the aim of which is to prevent further progression, or (ii) operative intervention, i.e. to remove the carious tooth substance and restore it with a filling. Although it is recommended that operative intervention should not be performed unless there is cavitation $^{31,32}$ or if radiographs show definite lesion progression over time ${ }^{8}$ stated treatment thresholds to intervene surgically differ among clinicians. ${ }^{33,34}$ For example, some clinicians would restore lesions restricted within the enamel in high-risk individuals. ${ }^{35}$ It may, however, be dubious to base a decision of operative intervention on caries risk assessment, as evidence concerning the validity of existing methods for identifying individuals at high risk is limited. ${ }^{36,6}$ 
We applied GRRAS, ${ }^{10}$ which outlines important issues to be addressed when studies on rater agreement are designed and reported. To the best of our knowledge, these guidelines have not previously been applied in studies published in dental journals. There is some overlap between GRRAS and the Standards for Reporting of Diagnostic Accuracy, ${ }^{37}$ which contains a checklist of essential elements to be included in diagnostic accuracy studies. In line with GRRAS, we reported agreement both as a percentage of agreement and as kappa statistics with CI to "allow the reader to get a detailed impression of the degree of the reliability and agreement". 10

The study has limitations. The study sample was selected by two of the authors in consensus. Although their assessment of caries lesion progression was not used as the reference standard, the selection of images affected the sample characteristics and thereby the results. ${ }^{38}$ We did not find any report on the frequency of caries lesion progression as assessed in bitewing radiographs in any selected sample or population. Nevertheless, the frequency of surfaces with caries lesion progression may have been higher in the present study than can be expected in a given patient population. On the other hand, the percentage agreement could be higher, when many sound surfaces are included. ${ }^{39}$ As people seem to be more influenced by representativeness

\section{References}

1. Schwendicke F, Tzschoppe M, Paris S. Radiographic caries detection: a systematic review and meta-analysis. J Dent $2015 ; 43$ 924-33. doi: http://dx.doi.org/10.1016/j.jdent.2015.02.009

2. Pliskin JS, Shwartz M, Gröndahl HG, Boffa J. Reliability of coding depth of approximal carious lesions from non-independent interpretation of serial bitewing radiographs. Community Dent Oral Epidemiol 1984; 12: 366-70. doi: http://dx.doi.org/10.111/ Oral Epidemiol 1984; 12: 366
j.1600-0528.1984.tb01473.x

j.1600-0528.1984.tb01473.x
3. Wenzel A. Computer-automated caries detection in digital bitewings: consistency of a program and its influence on observe agreement. Caries Res 2001; 35: 12-20. doi: http://dx.doi.org/ $10.1159 / 000047425$

4. Mejàre I, Axelsson S, Dahlén G, Espelid I, Norlund A, Svensson $\AA$, et al. Caries-diagnosis, risk assessment and non-invasive treatment. A systematic review. SBU-report no. 188. Summary and Conclusions in English. Stockholm, Sweden: Swedish Agency fo health Technology assessment (SBU); 2007. [Updated 2007; cited April 2016.] Available from: http://www.sbu.se/en/publications/sbuApril 2016. Available t

5. Senel B, Kamburoglu K, Uçok O, Yüksel SP, Ozen T, Avsever H. Diagnostic accuracy of different imaging modalities in detection of proximal caries. Dentomaxillofac Radiol 2010; 39: 501-11. doi http://dx.doi.org/10.1259/dmfr/28628723.

6. Senneby A, Mejàre I, Sahlin N-E, Svensäter G, Rohlin M. Diagnostic accuracy of different caries risk assessment methods. A systematic review. J Dent 2015; 43: 1385-93, doi: http://dx.doi. systematic review. J Dent 2015 ;
org/10.1016/j.jdent.2015.10.011

7. Wenzel A, Anthonisen PN, Juul MB. Reproducibility in the assessment of caries lesion behaviour: a comparison between conventional film and subtraction radiography. Caries Res 2000; 34 214-8. doi: http://dx.doi.org/10.1159/000016593

8. Wenzel A. Digital radiography and caries diagnosis. Dentomax illofac Radiol 1998; 27: 3-11. doi: http://dx.doi.org/10.1038/sj. dmfr.4600321 than probability when judging under uncertainty, ${ }^{40}$ the frequency of caries lesion progression may have had a modest effect on the rater assessments and thereby on the results of their agreement.

The raters were prompted to look for caries lesion progression in specific approximal surfaces. Under such a directed search condition, the rater is explicitly asked to rate the likelihood of a specific category at one highinformation region. This did not mimic the clinical situation where "free search" is the case. Furthermore, for the intrarater assessments, the raters were presented with the image pairs in the same order at both occasions. This might have led to them remembering parts of the sample.

\section{Conclusions}

We propose a novel classification system for the assessment of caries lesion progression in bitewing radiography. This system can be used to facilitate reporting and communication of changes of caries lesions and is applicable to clinical practice as well as to research of caries risk assessment and of outcomes of different caries interventions. It demonstrated substantial-to-moderate rater agreement, although further studies of different samples and under different conditions are warranted.

9. Swets JA, Pickett RM. Evaluation of diagnostic systems: methods from signal detection theory. New York, USA: Academic Press; 1982.

10. Kottner J, Audigé L, Brorson S, Donner A, Gajewski BJ, Hróbjartsson A, et al. Guidelines for Reporting Reliability and Agreement Studies (GRRAS) were proposed. Int J Nurs Stud 2011; 48: 661-71. doi: http://dx.doi.org/10.1016/j.ijnurstu.2011.01.016

11. Kottner J, Audigé L, Brorson S, Donner A, Gajeweski BJ, Hróbjartsson A, et al. Guidelines for reporting reliability and agreement studies (GRRAS) were proposed. J Clin Epidemiol 2011; 64: 96-106. doi: http://dx.doi.org/10.1016/j.jclinepi.2010.03.002.

12. Dicom digital imaging and communication in Medicine. Arlington, USA: National Electrical Manufacturers Association (NEMA). [Updated 15 October 2015; cited December 2015.] Available from: http://dicom.nema.org/

13. Assessment of Display Performance for Medical Imaging Systems. Durham, USA: American Association of Physics in Medicine AAPM Task group 18; [Updated 2006; Cited December 2015]. Available from: http://deckard.mc.duke.edu/ samei/tg18 (test images: TG18-CT-1k-01 and TG18-QC-1k-01)

14. Landis JR, Koch GG. The measurement of observer agreement for categorical data. Biometrics 1977; 33: 159-74. doi: http://dx. doi.org/10.2307/2529310

15. Koch G. Effect of sodium fluoride in dentifrice and mouth wash on incidence of dental caries in schoolchildren. Odontol Revy 1967; 18(Suppl. 12): 1-125.

16. Gröndahl HG, Hollender L, Malmcrona E, Sundquist B. Dental caries and restorations in teenagers. III. Social factors and attitudes in relation to dental status and caries increment among urban teenagers in Sweden. Swed Dent J 1977; 1: 107-14.

17. Espelid I, Tveit AB, Mjör IA, Eriksen HM, Fjelltveit A, Oiestad V. Systems for grading occlusal and approximal carious lesions. [In Norwegian.] Nor Tannlaegeforen Tid 1990; 100: 658-63.

18. Rimmer PA, Pitts NB. Effects of diagnostic threshold and overlapped approximal surfaces on reported caries status. Community 
Dent Oral Epidemiol 1991; 19: 205-12. doi: http://dx.doi.org/ 10.1111/j.1600-0528.1991.tb00147.x

19. Amarante E, Raadal M, Espelid I. Impact of diagnostic criteria on the prevalence of dental caries in Norwegian children aged 5 , 12 and 18 years. Community Dent Oral Epidemiol 1998; 26: 87-94 doi: http://dx.doi.org/10.1111/j.1600-0528.1998.tb01933.x

20. Ismail AI, Sohn W, Tellez M, Amaya A, Sen A, Hasson H, et al. The International Caries Detection and Assessment System (ICDAS): an integrated system for measuring dental caries. Community Dent Oral Epidemiol 2007; 35: 170-8. doi: http://dx. doi.org/10.1111/j.1600-0528.2007.00347.x

21. Benn DK. Diagnosis of caries progression from serial bitewings: a comparison between teachers and practitioners. Br Dent J 1993; 175: 26-32. doi: http://dx.doi.org/10.1038/sj.bdj.4808211

22. Pitts NB. Monitoring of caries progression in permanent and primary posterior approximal enamel by bitewing radiography. Community Dent Oral Epidemiol 1983; 11: 228-35. doi: http://dx. doi.org/10.1111/j.1600-0528.1983.tb01883 x

23. Benn DK. Radiographic caries diagnosis and monitoring. Dentomaxillofac Radiol 1994; 23: 69-72. doi: http://dx.doi.org/ $10.1259 / \mathrm{dmfr} .23 .2 .7835505$

24. Ismail AI, Pitts NB, Tellez M; Authors of the International Caries Classification and Management System (ICCMS). The International Caries Classification and Management System (ICCMS ${ }^{\mathrm{TM}}$ ). An example of a caries management pathway. BMC Oral Health 2015; 15 (Suppl. 1): S9. doi: http://dx.doi.org/10.1186/1472-6831-15-S1-S9.

25. Omnell KA. Quantitative roentgenologic studies on changes in mineral content of bone in vivo. Acta Radiol 1957; 48(Suppl. 148): 1-86.

26. Dore KL, Brooks LR, Weaver B, Norman GR. Influence of familiar features on diagnosis: instantiated features in an applied setting. J Exp Psychol Appl 2012; 18: 109-25. doi: http://dx.doi. org/10.1037/a0026539

27. Blesser B, Ozonoff D. A model for the radiologic process. Radiology 1972; 103: 515-21. doi: http://dx.doi.org/10.1148/103.3.515

28. Brehmer A, Brehmer B. What have we learned about human judgment from thirty years of policy capturing. In: Brehmer B, Joyce CRB, eds. Human judgment: the SJT view. Amsterdam, the Netherlands: Elsevier Science Publishers B.V.; 1988. pp. 75-114.

29. Norman GR, Coblentz CL, Brooks LR, Babcook CJ. Expertise in visual diagnosis: a review of the literature. Acad Med 1992; 67 (Suppl. 10): S78-83.
30. Hintze H, Frydenberg M, Wenzel A. Influence of number of surfaces and observers on statistical power in a multiobserver ROC radiographic caries detection study. Caries Res 2003; 37: 200-5, doi: http://dx doi.org/10.1159/000070445

31. Mejàre I, Stenlund $\mathrm{H}$, Zelezny-Holmlund $\mathrm{C}$. Caries incidence and lesion progression from adolescence to young adulthood: a prospective 15-year cohort study in Sweden. Caries Res 2004; 38: $130-41$.

32. Baelum V, Hintze H, Wenzel A, Danielsen B, Nyvad B. Implications of caries diagnostic strategies for clinical management decisions. Community Dent Oral Epidemiol 2012; 40: 257-66. do: http://dx.doi.org/10.1111/j.1600-0528.2011.00655.x

33. Baraba A, Domejean-Orliaguet S, Espelid I, Tveit AB, Miletic I. Survey of Croatian dentists' restorative treatment decisions on approximal caries lesions. Croat Med J 2010; 51: 509-14. doi http://dx.doi.org/10.3325/cmj.2010.51.509

34. Vidnes-Kopperud S, Tveit AB, Espelid I. Changes in the treatment concept for approximal caries from 1983 to 2009 in Norway. Caries Res 2011; 45: 113-20. doi: http://dx.doi.org/10.1159/ Caries Res

35. Kakudate N, Sumida F, Matsumoto Y, Manabe K, Yokoyama $\mathrm{Y}$, Gilbert GH, et al. Restorative treatment thresholds for proximal caries in dental PBRN. J Dent Res 2012; 91: 1202-8. doi: http://dx.doi.org/10.1177/0022034512464778

36. Tellez M, Gomez J, Pretty I, Ellwood R, Ismail AI. Evidence on existing caries risk assessment systems: are they predictive of future caries? Community Dent Oral Epidemiol 2013; 41: 67-78. doi http://dx.doi.org/10.1111/cdoe.12003

37. Bossuyt PM, Reitsma JB, Bruns DE, Gatsonis CA, Glasziou PP, Irwig LM, et al; Standards for Reporting of Diagnostic Accuracy. Towards complete and accurate reporting of studies of diagnostic accuracy: the STARD initiative. Clin Radiol 2003; 58: 575-80. doi: http://dx.doi.org/10.1016/S0009-9260(03)00258-7

38. Viera AJ, Garrett JM. Understanding interobserver agreement: the kappa statistic. Fam Med 2005; 37: 360-3.

39. de Paola PF, Alman J. Assessment of the reliability of radiographic diagnosis in a clinical caries trial. J Dent Res 1972; 51: 1431-7. doi: http://dx.doi.org/10.1177/00220345720510053001

40. Tversky A, Kahneman D. Judgment under uncertainty: heuristics and biases. Science 1974; 185: 1124-31. doi: http://dx.doi.org/ 10.1126/science. 185.4157 .1124 
Supplementary Table 1 GRRAS checklist for reporting of studies of reliability and agreement ${ }^{11}$ with modifications

\begin{tabular}{|c|c|c|c|c|}
\hline Section & Item \# & Checklist Item & Modifications & Comment(s) \\
\hline Title, Abstract & 1. & $\begin{array}{l}\text { Identify in title or abstract that } \\
\text { interrater/intrarater reliability or agreement } \\
\text { was investigated }\end{array}$ & Yes & $\begin{array}{l}\text { The primary aim was to design a } \\
\text { classification system. Thus, rater } \\
\text { agreement is not mentioned in } \\
\text { title but in abstract }\end{array}$ \\
\hline \multirow[t]{4}{*}{ Introduction } & 2. & $\begin{array}{l}\text { Name and describe the diagnostic or } \\
\text { measurement device of interest explicitly }\end{array}$ & - & \\
\hline & 3. & Specify the subject population of interest & - & \\
\hline & 4. & $\begin{array}{l}\text { Specify the rater population of interest (if } \\
\text { applicable) }\end{array}$ & - & \\
\hline & 5. & $\begin{array}{l}\text { Describe what is already known about } \\
\text { reliability and agreement and provide a } \\
\text { rationale for the study (if applicable) }\end{array}$ & - & \\
\hline \multirow[t]{5}{*}{$\begin{array}{l}\text { Material and } \\
\text { Methods }\end{array}$} & 6. & $\begin{array}{l}\text { Explain how the sample size was chosen. } \\
\text { State the determined number of raters, } \\
\text { subjects/objects, and replicate observations }\end{array}$ & Yes & $\begin{array}{l}\text { The choice of sample size is not } \\
\text { explained in Material and } \\
\text { Methods but is elucidated in } \\
\text { Discussion }\end{array}$ \\
\hline & 7. & Describe the sampling method & - & \\
\hline & 8. & $\begin{array}{l}\text { Describe the measurements/rating process } \\
\text { (e.g., time interval between repeated } \\
\text { measurements, availability of clinical } \\
\text { information, blinding) }\end{array}$ & - & \\
\hline & 9. & $\begin{array}{l}\text { State whether measurements/ratings were } \\
\text { conducted independently }\end{array}$ & - & \\
\hline & 10. & Describe the statistical analysis & - & \\
\hline \multirow[t]{4}{*}{ Results } & 11. & $\begin{array}{l}\text { State the actual number of raters and } \\
\text { subjects/objects that were included and the } \\
\text { number of replicate observations that were } \\
\text { conducted }\end{array}$ & Yes & $\begin{array}{l}\text { Number of replicate observations } \\
\text { reported in Material and Methods }\end{array}$ \\
\hline & 12. & $\begin{array}{l}\text { Describe the sample characteristics of raters } \\
\text { and subjects (e.g., training, experience) }\end{array}$ & Yes & $\begin{array}{l}\text { Reported in section Material and } \\
\text { Methods }\end{array}$ \\
\hline & 13. & $\begin{array}{l}\text { Report estimates of reliability and agreement } \\
\text { including measures of statistical uncertainty }\end{array}$ & - & \\
\hline & 14. & Discuss the practical relevance of results & - & \\
\hline $\begin{array}{l}\text { Auxiliary } \\
\text { material }\end{array}$ & 15. & $\begin{array}{l}\text { Provide detailed results if possible (e.g., } \\
\text { online) }\end{array}$ & Yes & $\begin{array}{l}\text { Raw data on raters assessments } \\
\text { not presented online }\end{array}$ \\
\hline
\end{tabular}

GRRAS-checklist available at http://www.equator-network.org/reporting-guidelines/guidelines-for-reporting-

reliability-and-agreement-studies-grras-were-proposed/. 

III 



\title{
Acid Tolerance Properties of Dental Biofilms in vivo
}

\author{
Senneby A $A^{\mathrm{a}}$, Davies JR ${ }^{\mathrm{b}}$, Svensäter $\mathrm{G}^{\mathrm{b}}$, Neilands $\mathrm{J}^{\mathrm{b}}$ \\ a Department of Oral and Maxillofacial Radiology and ${ }^{b}$ Department of Oral Biology, Faculty \\ of Odontology, Malmö University, Malmö, Sweden
}

Correspondence should be addressed to:

Jessica Neilands

Malmö University, Faculty of Odontology, SE-205 06 Malmö, Sweden

Phone number: +46 709163515 or +46 406658494

E-mail: jessica.neilands@mah.se

Declaration of interests: No conflict of interests 


\begin{abstract}
Background: The ecological plaque hypothesis explains caries development as the result of the enrichment of acid tolerant bacteria in dental biofilms in response to prolonged periods of low $\mathrm{pH}$. Acid production by an acid tolerant microflora causes demineralisation of tooth enamel and thus, individuals with a greater proportion of acid tolerant bacteria would be expected to be more prone to caries development. Biofilm acid tolerance could therefore be a possible biomarker for caries prediction. However, little is known about the stability of biofilm acid tolerance over time in vivo or the distribution throughout the oral cavity. Therfore the aim of this study was to assess intra-individual differences in biofilm acid-tolerance between different tooth surfaces and inter-individual variation as well as stability of acid tolerance over time.
\end{abstract}

Methods: Biofilms were collected from approximal surfaces of canines and $1^{\text {st }}$ premolars, $2^{\text {nd }}$ premolar and $1^{\text {st }}$ molars, and between the central incisors in adolescents at baseline, day 3 , after 1-, 6- and 12 months. After exposure of the biofilm cells to $\mathrm{pH} 3.5$ for $2 \mathrm{~h}$ the cells were visualized using LiveDead ${ }^{\circledR}$ BacLight ${ }^{\mathrm{TM}}$ staining and CSLM. The acid tolerance was then assessed as the percentage bacteria that survived this treatment in each sample by comparison of the proportion of live bacteria in the images with a prepared scale.

Results: The majority of the adolescents showed low scores for biofilm acid tolerance. In 14 of 20 individuals no differences were seen between the three sites examined. In the remaining six, acid-tolerance at the premolar site differed from one of the other sites. At 51 of 60 sites, acid-tolerance at baseline was unchanged after one month. However, acid tolerance values changed over a 1 -year period in $50 \%$ of the individuals.

Conclusions: Biofilm acid tolerance showed short-term stability and low variation between different sites in the same individual suggesting that the acid tolerance could be a promising biological biomarker candidate for caries prediction. Further evaluation is however needed and prospective clinical trials are called for to evaluate the diagnostic accuracy.

Key words: Microbiology, Phenotype, Plaque, $\mathrm{pH}$, Observer performance, Acid tolerance 


\section{Introduction}

The oral microbiota plays an important role in health by preventing colonisation of the oral cavity by pathogenic species [1]. The human oral cavity exhibits a high degree of bacterial diversity and more than 700 taxa have been identified in meta-genomic studies [2]. Bacteria grow in complex multi-species biofilms on the hard and soft-oral tissue surfaces with saliva or gingival exudate as the major nutrient sources. Biofilms on the teeth (dental plaque) above the gingival margin are dominated by sacharolytic bacteria, which generate energy by breakdown of carbohydrates from salivary glycoproteins as well as ingested food, through the glycolytic pathway. Pyruvate conversion then results in acidic end-products, including lactic acid, which can rapidly lower the $\mathrm{pH}$ in dental biofilms. If the $\mathrm{pH}$ remains lower than 5.5 for a prolonged period, demineralisation of the enamel will occur $[3,4]$.

As well as causing demineralisation, acids generated from carbohydrate metabolism by members of the biofilm affect the ecology of the biofilm itself. Prolonged periods of low $\mathrm{pH}$ in biofilms favour growth of intrinsically acid-tolerant (aciduric) bacteria such as Lactobacilli and Bifidobacteria, leading to increased proportions of these species [5]. Experiments using the model bacterium Streptococcus mutans have shown that, although not intrinsically aciduric, oral streptococci can survive acid stress through the induction of an acid-tolerance response (ATR) when exposed to a sub-lethal $\mathrm{pH}(\sim \mathrm{pH} 5)[6,7]$. Acid tolerance is the ability of a bacterium to sense and respond to acid stress [8] and is a phenomenon first discovered in Salmonella enterica serovar typhimurium [9]. Later it has been shown to occur in several other species including Escherichia coli, Listeria monocytogenes, Bifidobacterium longum and oral bacteria such as Enterococcus hirae, Streptococcus gordonii, Streptococcus sanguinis and Lactobacillus casei $[7,10,11,12,13]$. The ATR in $S$. mutans involves, for example, changes in cell membrane composition, exclusion and extrusion of protons from the cytoplasm, generation of alkali from arginine-containing proteins and up-regulation of chaperones and nucleases to prevent protein misfolding and maintain DNA integrity respectively. $[6,14,15$, $16,17,18]$. In addition to these survival mechanisms, a shift in the $\mathrm{pH}$ optimum for glucose transport and glycolysis allows acid-tolerant streptococcal cells to continue metabolising carbohydrates and producing acid end-products even in acidic environments [6]. Thus, frequent exposure to low $\mathrm{pH}$ will favour, not only intrinsically aciduric species, but also bacteria capable of eliciting an ATR [1, 19]. Selection of intrinsically and adapted acid-tolerant bacteria eventually results in a highly aciduric plaque that promotes demineralization of enamel [20] 
and development of caries lesions [1, 21, 22, 23, 24]. Consequently, individuals with highly aciduric plaque would be expected to have an increased risk of developing caries compared to those within non-aciduric biofilms.

Caries is one of the most common biofilm-mediated diseases in the world and around $10 \%$ of the population worldwide suffer from severe disease. In order to target preventative measures most effectively, it is essential to be able to identify those individuals with increased risk of developing severe caries. Traditionally, methods for caries risk assessment include previous caries experience, sociodemographic- or socio-economic factors, salivary secretion rate and buffer capacity as well as oral hygiene parameters [25-27]. In addition, microbiological tests that measure the number of mutans streptococci or lactobacilli in saliva or plaque are used. These tests show high specificity but low and scattered sensitivity [25-28]. Thus, individuals who are not at risk of developing caries are effectively identified whereas those with increased risk are not. There are several possible reasons for the low sensitivity seen for tests that rely on the count of specific bacterial species, (e.g. S. mutans). For example, it is well known that dental plaque harbours other streptococci that are capable of exhibiting an acid tolerant phenotype (e.g. S. gordonii and Streptococcus oralis) that would lead to a false negative result with this kind of test [20]. On the contrary, the presence of non-aciduric $S$. mutans in the sample would give rise to false positive results. The low sensitivity of existing tests for caries prediction, clearly illustrates a need for new approaches.

Attempts have been made to develop methods that detect the actual level of acid-tolerance in dental plaque. These include enumeration of bacteria after culturing on low $\mathrm{pH}$ agar, where only aciduric bacteria can grow [29] and measurement of growth in acidified broth in microtiter plates [30]. Another method of distinguishing acid-tolerant bacteria from non-acid-tolerant ones is by exposing an established biofilm (dental plaque sample) to an acid challenge (i.e. a pH known to kill non-acid-tolerant bacteria) and visually assessing the proportion of bacteria that survive by staining with the LIVE/DEAD® BacLight ${ }^{\mathrm{TM}}$ stain [31]. The latter method has been used previously to determine the levels of acid-tolerant bacteria in dental plaque samples from exposed root surfaces in elderly patients [32]. Since the presence of acid tolerant microorganisms in biofilms is intimately related to the demineralisation process of the tooth, the acid challenge method could be a possible test in caries prediction. However in order to be a possible candidate it must not fluctuate over the short term and preferably show low variation within the oral cavity so that sampling can be easy and representative. In this study we have 
used the acid challenge method to investigate biofilm acid tolerance at different tooth sites in the same individual as well as the variation between individuals. In addition, short and longterm changes in acid tolerance have been studied. This work represents the first step in the evaluation process of biofilm acid tolerance in vivo as a possible biomarker in caries prediction.

\section{Material and Methods}

\section{Subjects}

Forty adolescents (aged 12-13 years) visiting a public dental health clinic in Kronoberg County, Sweden for their regular dental check-up appointment were recruited to the study. Ethical approval was obtained from the Regional Ethical Review Board, Lund, Sweden (registration number: 2016/146). Adolescents and parents received written information by post prior to the visit and informed consent was obtained before enrollment in the study. Eligible subjects were healthy individuals with erupted permanent first molars, first premolars and incisors. Individuals who had received antibiotic treatment over the past 3 months were excluded.

\section{Sample Collection}

Plaque biofilm sampling was performed by a dental hygienist, who was trained in the procedure. For half of the adolescents $(n=20)$ biofilms were sampled using Quicksticks (Dab Dental AB, Upplands Väsby, Sweden) from all supragingival approximal surfaces between second premolars and first molars (four sites) and pooled to give one sample for each individual. Samples were taken at baseline (time 0), after 6 and 12 months. In the other individuals ( $\mathrm{n}=$ 20) biofilms were sampled and pooled in the same manner from all approximal surfaces between second premolars and first molars (four sites), between canines and first premolars (four sites) and between the central incisors in both jaws (two sites) resulting in three samples from each individual. This sampling took place at baseline, after three days and after one month. Samples were transferred to sterile microfuge tubes and sent to the laboratory for analysis.

\section{Assessment of Biofilm Acid Tolerance}

Each sample was suspended in $200 \mu 1$ TYE medium (1.7\% tryptone, $0.3 \%$ yeast) containing 40 $\mathrm{mM}$ phosphate/citrate (P/C) buffer ( $\mathrm{pH} 3.5)$ and $20 \mathrm{mM}$ glucose and vortexed with glass beads to disperse the biofilm before incubation at $37^{\circ} \mathrm{C}$ for 2 hours. Cells were then stained using 
LIVE/DEAD ${ }^{\circledR}$ BacLight $^{\mathrm{TM}}$ Fluorescent Stain (Molecular Probes, Eugene, USA) [31] and introduced into Ibidi mini flow cells (IbidiR $\mu$-Slide, Ibidi GmbH, Martinsried, Germany). Each sample was examined using an inverted confocal scanning laser microscope (Nikon Eclipse TE2000, Nikon Corp., Tokyo, Japan) with an Ar laser (488 nm laser excitation). Live (acidtolerant) bacteria appeared green while dead (non acid-tolerant) bacteria appeared red (Figure 1). Assessment of acid-tolerance was based on scoring of ten random images from each sample. Due to differences in bacterial size and cellular morphology, it was not possible to estimate the percentage of green (acid-tolerant) and red (non-acid-tolerant) bacteria using pixel-based methods. Assessment was therefore carried out by an experienced rater (author JN) through comparison of the proportion of green cells in each image with an interval scale (with 5 possible scores or threshold values) presented in Figure 1 [32]. A change in the level of acid-tolerance was defined as an increase or decrease of $\geq 2$ scores according to the interval scale. This corresponded to a difference of at least $20 \%$ in the proportion of acid-tolerant cells in the images. Scoring is more time efficient than counting the cells in each image manually, especially when a large number of images are to be analysed. To test the validity of the interval scale (1-5 scores) for assessment of the proportion of acid-tolerant cells in biofilms, a comparison was made of the score given by the rater with that given by counting the actual numbers of acid-tolerant cells in 10 images ( 2 images representing each score). The intra-rater agreement of acid-tolerance assessments according to the interval scale was determined by comparing the scores given for the same 50 images, presented in random order, on two separate occasions separated by two weeks. The 50 images represented 10 images for each score.

\section{Results}

Validity of interval scale for assessment of acid-tolerance in biofilms

Acid tolerance in this study was defined as the proportion of biofilm cells that were green (i.e. viable following an acid challenge ( $\mathrm{pH} 3.5$ ) for two hours) by visual comparison with the scale shown in Figure 1. For eight images, the score assigned using the scale was exactly the same as that revealed by counting. For two images however, the rater's assessment differed by one score from that obtained by counting. In both images, the actual number of acid-tolerant cells was close to the threshold boundary, for example, an image given score 3 by the rater (corresponding to $41-60 \%$ acid-tolerant cells) actually contained $62 \%$ acid-tolerant cells. 
The intra-rater agreement was $94 \%$ (kappa 0.92 ) as the exact same score was given on the two occasions. In the remaining 3 images, the results differed by one score (defined as no difference). Thus, the intra-rater agreement according to Landis and Koch was almost perfect [33].

\section{Distribution of acid-tolerance in 12-year old individuals}

To determine the range of acid-tolerance scores within the study population, biofilm samples were taken on a single occasion from the approximal surfaces between the second pre-molar and first molar in each quadrant and pooled to give one sample from each individual. This revealed a spread in biofilm acid-tolerance amongst the 40 individuals over the entire range of the evaluation scale (from score $1,0-20 \%$ to score 5, 81-100\%). Most of the individuals (75\%) had a low acid-tolerance (score 1 or 2 ) while less than $10 \%$ had biofilms with a high acidtolerance (score 4 or 5) (Figure 2). Thus, the level of acid tolerance within the study population varied, with the majority of individuals showing low levels of acid-tolerance in their oral biofilms. In images of biofilms from the individuals showing a high acid tolerance, both green cocci and short, long and pleomorphic, green rods could be observed, suggesting that a range of different bacterial species in the biofilms were acid-tolerant (Figure 3).

\section{Comparison of Acid Tolerance between Different Tooth Surfaces in the same Individual}

To investigate whether acid-tolerance varied between tooth surfaces in the same individual, biofilms were sampled on a single occasion from three different approximal sites in 20 subjects. In all individuals, the sample taken from between the first incisors and that taken between the second premolar and the first molar showed exactly the same acid-tolerance score. In fourteen, the approximal site between the canines and the first premolar had the same acid-tolerance score as the incisor and molar sites. However, in the remaining six individuals, the acid-tolerance at the pre-molar site differed from one of the other sites (Table 1).

\section{Reliability of the acid tolerance assessment method}

To investigate the reliability of the assessment method, sampling from between the central incisors, canine and first premolar, and second premolar and first molar sites was repeated after three days and the acid-tolerance values compared with the baseline measurements. This revealed a high degree of short-term consistency, with the same values obtained in $90 \%$ of the individuals at the incisor and molar sites and $85 \%$ of the individuals at the sites between the canines and pre-molars. 


\section{Changes in biofilm acid-tolerance over time}

To investigate the time-frame over which changes in biofilm acid-tolerance occurred, the values at baseline at 60 sites were compared with those obtained after 1 month. At fifty-one of sixty sites, low (score 1 and 2) or moderate (score 3) acid-tolerance values at baseline were unchanged after one month (Table 2). At four sites a substantial increase in acid-tolerance was seen, whereas at five sites a substantial decrease was seen. These data suggest that the proportion of acid-tolerant organisms in biofilms can both increase and decrease over one month.

Since we postulate that caries development would require a high-degree of acid tolerance in the biofilm over a considerable period, fluctuation in biofilm acid-tolerance at one site over the longer term (six months to one year) was also studied. Eighty-five percent of the sites showed a low or moderate acid-tolerance at baseline and the values fluctuated around this level over the one-year period (Table 3). At two sites however, a high acid-tolerance measurement at baseline (score 5) decreased to a low value at 6 months (score 1), which was maintained at the one-year sampling point. In contrast, at 1 site the opposite occurred with a low initial acid tolerance score increasing to a high score after 6 months that then remained stable at one year. These results show that for most individuals in this study, acid tolerance is generally low and stable over the long-term. However, a small proportion of the individuals showed changes between the values obtained on the first and second sampling occasions and that once a change had occurred, the new level of acid tolerance was maintained at the one-year sampling point.

\section{Discussion}

Supragingival plaque biofilms develop through adherence of microorganisms to saliva-coated tooth surfaces. These biofilms show a high degree of diversity but are dominated by Streptococcus and Actinomyces, which express adhesins that can bind adsorbed salivary proteins. Both these genera are saccharolytic and normally acquire nutrients through the cooperative degradation of glycan chains from salivary glycoproteins. Intermittently carbohydrates derived from food are metabolized by microorganisms, giving rise to products such as acetate, lactate, formate and succinate, which lead to rapid acidification of the biofilm [3]. As early as 1944, Stephan [34] showed that the $\mathrm{pH}$ in plaque from caries-active individuals was initially lower and fell to a lower level for a longer period in response to a glucose rinse, 
than in plaque from healthy subjects. The phenomenon has been confirmed in subsequent studies; see for instance Aranibar Quiroz et al. [35], where, in caries-active individuals, biofilm $\mathrm{pH}$ remained below 5.5 for more than 15 minutes after a sucrose rinse. This observation has been interpreted as evidence that biofilms showing sustained $\mathrm{pH}$ drops contain acid-tolerant bacteria, which survive the long-term acidic environment and are able to metabolize and generate acid at low $\mathrm{pH}$ levels [36]. In addition to adapted acid-tolerant species such as members of the genera Streptococcus and Actinomyces acid tolerant bacteria also include the intrinsically acid-tolerant genera such as Lactobacillus [23] and Bifidobacterium [37]. Although S. mutans and Streptococcus sobrinus were the first acid-tolerant streptococcal species identified, today it is known that other streptococci such as S. oralis, Streptococcus mitis, $S$. sanguinis and Streptococcus anginosus also can exhibit acid-tolerance and indeed S. oralis can produce acid at rates exceeding that of some $S$. mutans strains $[7,20,30,38]$. In this study, images of plaque biofilms from individuals with a high acid-tolerance score revealed a range of bacterial morphologies amongst the acid-tolerant cells, indicating that a variety of bacterial species probably contribute to the overall acid-tolerant microbiota of the biofilm.

Irrespective of the specific bacterial composition, a high degree of acid tolerance would enhance the cariogenic potential of the supragingival biofilm and the appearance of an acid-tolerant microbiota would be expected to be an early event in the caries process. Thus, it may be possible to utilize evaluation of the overall level of acid tolerance in oral biofilms as an early marker to predict enamel caries. In this study, the proportion of acid-tolerant microorganisms in biofilms collected from the study population ranged from low to high on the scale (Figure 2), confirming that the method has the capacity to distinguish between individuals with different levels of biofilm acid-tolerance. The majority (92\%) of the subjects showed low levels (scores 1-3) suggesting that they lack the pre-requisite conditions necessary for development of caries and would be expected to have a low risk for disease development. This figure corresponds well to the proportion of caries-free 12-year olds in the Health Authority area [39]. On the other hand, $8 \%$ had a high level of acid-tolerant bacteria (score 4 or 5), suggesting that these individuals may have an increased risk of developing caries.

The oral cavity is known to contain a number of distinct ecological niches influenced by factors such as nutrient supply, $\mathrm{pH}$ and oxygen tension. For instance, the properties of subgingival biofilms are known to differ from those of biofilms above the gingival margin or on the tongue. To investigate whether there are differences between supragingival sites within subjects, acid 
tolerance in approximal plaque from the incisor, pre-molar and molar regions was compared. The acid-tolerance was shown to be largely consistent for the three selected sites within an individual; with complete agreement between the values for the incisor and molar regions. This suggests that supragingival biofilms on different teeth are subjected to the same stress factors and ecological influences of relevance for acid-tolerance development [1].

To investigate the reliability of the assessment method for acid-tolerance of the biofilms, two samples were taken from the same site 3 days apart. In 19 of 20 individuals, biofilm acid tolerance was shown to be low at baseline and the same outcome was seen after 3 days. This confirms that the method is reliable since detectable changes in acid-tolerance are unlikely to occur during this time. Score 4 and 5 represents a dysbiotic state indicating high carbohydrate intake and low $\mathrm{pH}$ over time. A pre-requisite for the development of an acid tolerant microbiota is acid-adaptation and the enrichment of aciduric strains within the biofilm [40] and since bacterial doubling time in dental biofilms in vivo is known to be in excess of 21 hours, it is unlikely that acid-tolerant bacteria would achieve numerical dominance in the biofilm over 3 days. It is therefore also unlikely that any food intake prior to sampling would affect the result. However, to investigate more closely how rapidly changes in biofilm acid tolerance can occur, samples were retaken from the same site after 1 month. At 51 of 60 sites, the acid-tolerance at baseline was low or moderate and no changes were seen between samples taken at baseline and at one month. However, the acid tolerance increased at 4 and decreased at 5 sites over the 1month period suggesting that both acid-adaptation as well as de-adaptation can occur over this time. Acid adaptation has previously been demonstrated to occur over 10 days in in vitro experiments in chemostats using a 9-species-consortium that was pulsed daily with glucose [41].

At present there are no marker-based methods to identify acid tolerant bacteria. However, exposing bacteria to low $\mathrm{pH}$ is commonly used to distinguish acid tolerant from non-acid tolerant ones. This method is based on the ATR concept - where an exposure to sub-lethal $\mathrm{pH}$ values $(\mathrm{pH} 5.5)$ leads to an ATR that enhances survival at $\mathrm{pH}$ values which kill non-adapted cells (killing $\mathrm{pH}$ ) [9]. Bacteria that have been exposed to sub-lethal $\mathrm{pH}$ in the oral cavity due to metabolism of carbohydrates would be acid-adapted and thus when exposed to a killing $\mathrm{pH}(\mathrm{pH}$ 3.5) ex vivo these adapted bacteria as well as intrinsically acid tolerant ones will survive whereas not acid tolerant will not. The killing $\mathrm{pH}$ was chosen based on previous studies on acid tolerance capacity of different oral species [7]. Acid tolerant bacteria (defined as viable cells after the ex 
vivo $\mathrm{pH}$ challenge) were distinguished from non-acid tolerant ones using the LIVE/DEAD ${ }^{\circledR}$ BacLight $^{\mathrm{TM}}$ Viability stain. The LIVE/DEAD stain have been tested on a range of different Gram positive and Gram negative species and have shown good correlation with standard plate counts [42].

In order for enamel caries lesions to develop and progress, extended periods of low $\mathrm{pH}$ within biofilms are required. Studies have shown that during cariogenic challenge caries lesions can develop within 14 days although under normal circumstances caries development and progression is a much slower process [43-45]. Therefore, acid-tolerance in biofilms within the subjects over 1 year was also investigated. As seen in the short-term investigation, in the majority of individuals the acid-tolerance at baseline was low and this was maintained at 6 months and 1 year. However, in a minority of subjects a major difference was seen between the level of acid tolerance and baseline and that at 6 months. In two individuals, the reverse appeared to have occurred where high acid tolerance levels at baseline were found to be low at both 6 months and 1 year. Thus succession towards a healthier microbiota appears to have occurred in these individuals, suggesting that the ecological pressure driving development of acid tolerance has been removed in these subjects. In one individual, a low acid tolerance score at baseline had increased to a high score at six months, which was also seen at 1 year. This suggests that the normal homeostasis within the biofilm in this subject was disrupted leading to extended periods of low $\mathrm{pH}$ and succession of acid-tolerant bacteria. Factors potentially driving this process could be frequent intake of fermentable carbohydrates or reduced buffer capacity in saliva.

The acid tolerance test is an indicator of the acid-producing potential of a biofilm and is thus intimately related to the demineralisation process. As such it represents a promising biological biomarker candidate for caries prediction. However further evaluation of the test is needed and prospective clinical trials are called for to evaluate the diagnostic accuracy.

\section{Acknowledgements}

We would like to thank Maria Hilding and Christina Stebring-Franzon at the Public Dental Health Clinic in Alvesta, Sweden and Dr Ulrika Troedsson for their help with this project. 


\section{Funding}

The work was funded by the Borrow Foundation, UK [Charity No. 1060308] and The Swedish Research Council [2016-01994].

\section{Availability of data and materials}

Not applicable

\section{Author's contribution}

AS and JN performed experiments and the acquisition of the data. AS, JN and GS analyzed the data. AS, JRD, GS, and JN wrote the paper and revised the work for intellectual content. All authors gave a final approval of the work to be published and agree to be accountable for all aspects of the work.

\section{Competing interest}

The authors declare that they have no competing interests.

\section{Consent for publication}

Not applicable

\section{Ethics approval and consent to participate}

Ethical approval was obtained from the Regional Ethical Review Board, Lund, Sweden (registration number: 2016/146). Adolescents and parents received written information by post prior to the visit and informed consent was obtained before enrollment in the study. 


\section{References}

1. Marsh PD. Microbial ecology of dental plaque and its significance in health and disease. Adv Dent Res. 1994;8:263-71.

2. Jenkinson HF. Beyond the oral microbiome. Environ Microbiol. 2011;13:3077-87.

3. Carlsson J, Hamilton IR. Metabolic activities of oral bacteria. In: Thylstrup A, Fejerskov O, editors. Textbook of clinical cariology. 2nd ed. Copenhagen: Munksgaard; 1994. p 71-110.

4. Bowden GHW. Which bacteria are cariogenic in humans? In: N.M. Johnson, editor. Dental Caries Vol. 1: Markers of High and Low Risk Groups and Individuals. Cambridge: Cambridge University Press; 1991. p. 266-86.

5. Marsh PD. Microbial ecology of dental plaque and its significance in health and disease. Adv Dent Res 1994;8:263-71.

6. Hamilton IR, Buckley ND. Adaptation by Streptococcus mutans to acid tolerance. Oral Microbiol Immunol. 1991;6:65-71.

7. Svensäter G, Larsson U-B, Greif ECG, Cvitkovitch DG, Hamilton IR. Acid tolerance response and survival by oral bacteria. Oral Microbiol Immunol. 1997;12:266-73.

8. Foster JW. Microbial responses to acid stress. In: Storz G, Hengge-Aronis R, editors. Bacterial stress responses. Washington DC: ASM Press. 2000. p.99-115.

9. Foster JW, Hall HK. Adaptive acidification tolerance response of Salmonella typhimurium. J Bacteriol. 1990;172:771-8.

10. Foster JW, Moreno M. 1999. Inducible acid tolerance mechanisms in enteric bacteria. Novartis Found Symp. 1999; 221:55-69.

11. Gahan CG, O'Driscoll B, Hill C. Acid adaptation of Listeria monocytogenes can enhance survival in acidic foods and during milk fermentation. Appl Environ Microbiol. 1996;62:312832.

12. Takahashi N, Xiao JZ, Miyaji K, Yaeshiima T, Hiramatsu A, Iwatsuki K, Kokubo $\mathrm{S}$, Hosono A. Selection of acid tolerant bifidobacteria and evidence for a low-pHinducible acid tolerance response in Bifidobacterium longum. J Dairy Res. 2004;71:340-5.

13. Belli WA, Marquis RE. Adaptation of Streptococcus mutans and Enterococcus hirae to acid stress in continuous culture. Appl Environ Microbil. 1991;57:1134-8.

14. Quivey RG, Faustoferri RK Jr, Monahan K, Marquis RE. Shifts in membrane fatty acid profiles associated with acid adaptation of Streptococcus mutans. FEMS Microbiol Lett. 2000;189:89-92. 
15. Fozo EM, Quivey RG Jr. Shifts in the membrane fatty acid profile of Streptococcus mutans enhance survival in acidic environments. Appl Environ Microbiol. 2004;70:929-36.

16. Lemos JA, Abranches J, Burne RA. Responses of cariogenic streptococci to environmental stresses. Curr Issues Mol Biol. 2005;7:95-107.

17. Burne RA, Marquis RE. Alkali production by oral bacteria and protection against dental caries. FEMS Microbiol Lett. 2000;192:1-6.

18. Sturr MG, Marquis RE. Comparative acid tolerances and inhibitor sensitivities of isolated F-ATPases of oral lactic acid bacteria. Appl Environ Microbiol. 1992;58:2287-91.

19. Bradshaw DJ, McKee AS, Marsh PD. Effect of carbohydrate pulses and $\mathrm{pH}$ on population shifts within oral microbial communities in vitro. J Dent Res. 1989;68:1298-1302.

20. de Soet JJ, Nyvad B, Kilian M. Strain-related acid production by oral streptococci. Caries Res. 2000 Nov-Dec;34:486-90.

21. van Houte J. Role of micro-organisms in caries etiology. J Dent Res. 1994;73:672-81.

22. de Soet JJ1, Toors FA, de Graaff J. Acidogenesis by oral streptococci at different $\mathrm{pH}$ values. Caries Res. 1989;23:14-7.

23. Harper DS, Loesche WJ. Growth and acid tolerance of human dental plaque bacteria. Arch Oral Biol. 1984;29:843-8.

24. Featherstone JD, Duncan JF, Cutress TW. A mechanism for dental caries based on chemical processes and diffusion phenomena during in-vitro caries simulation on human tooth enamel. Arch Oral Biol. 1979;24:101-12.

25. Schwendicke F, Tzschoppe M, Paris S. Radiographic caries detection: A systematic review and meta-analysis. J Dent. 2015;43:924-33.

26. Mejàre I, Axelsson S, Dahlén G, Espelid I, Norlund A, Tranæus S, Twetman S. Caries risk assessment. A systematic review. Acta Odontol Scand. 2014;72:81-91.

27. Mejàre I, Axelsson S, Espelid I, Dahlén G, Norlund A, Svensson Å et al. Caries - diagnosis, risk assessment and non-invasive treatment. A systematic review. Summary and Conclusions. Stockholm: The Swedish Council on Technology Assessment in Health Care, report no: 188; 2007. http://www.sbu.se/upload/Publikationer/Content1/1/Caries summary 2008.pdf. (cited 12-22-16).

28. Senneby A, Mejàre I, Sahlin NE, Svensäter G, Rohlin M. Diagnostic accuracy of different caries risk assessment methods. A systematic review. J Dent. 2015;43:1385-93.

29. Svensäter G, Borgström M, Bowden GH, Edwardsson S. The acid-tolerant microbiota associated with plaque from initial caries and healthy tooth surfaces. Caries Res 2003;37:395403. 
30. Brailsford SR, Shah B, Simons D, Gilbert S, Clark D, Ines I, Adams SE, Allison C, Beighton

D. The predominant aciduric microflora of root-caries lesions. J Dent Res. 2001;80:1828-33.

31. Welin-Neilands J, Svensäter G. Acid tolerance of biofilm cells of Streptococcus mutans. Appl Environ Microbiol. 2007;73:5633-38.

32. Neilands J, Petersson LG, Beighton D, Svensäter G. Fluoride-supplemented milk inhibits acid tolerance in root caries biofilms. Caries Res. 2012;46:156-60.

33. Landis JR, Koch GG. The measurement of observer agreement for categorical data. Biometrics 1977;33:159-74..

34. Stephan RM. Intra-oral hydrogen-ion concentration associated with dental caries activity. J Dent Res. 1944;23:257-66.

35. Aranibar Quiroz EM, Alstad T, Campus G, Birkhed D, Lingström P. Relationship between plaque $\mathrm{pH}$ and different caries-associated variables in a group of adolescents with varying caries prevalence. Caries Res. 2014;48:147-53.

36. Bowden GH, Hamilton IR. Survival of oral bacteria. Crit Rev Oral Biol Med. 1998;9:5485.

37. Mantzourani M, Gilbert SC, Sulong HN, Sheehy EC, Tank S, Fenlon M, Beighton D. The isolation of bifidobacteria from occlusal carious lesions in children and adults. Caries Res. 2009;43:308-13.

38. Takahashi N, Yamada T. Acid-induced acid tolerance and acidogenicity of non-mutans streptococci. Oral Microbiol Immunol. 1999;14:43-8.

39. National Board of Health and Welfare. Caries in children and adolescents. https://www.socialstyrelsen.se/Lists/Artikelkatalog/Attachments/19772/2015-3-20.pdf （in Swedish) (cited Dec 27, 2016).

40. Marsh PD. Are dental diseases examples of ecological catastrophes? Microbiology 2003;149:279-94.

41. Bradshaw DJ, Marsh PD. Analysis of pH-driven disruption of oral microbial communities in vitro. Caries Res. 1998;32:456-62.

42. Davies CM. A comparison of fluorochromes for direct viable counts by image analysis. Lett Appl Microbil. 1991;13:58-61.

43. Mejàre I, Källest IC, Stenlund H. Incidence and progression of approximal caries from 11 to 22 years of age in Sweden: A prospective radiographic study. Caries Res 1999;33:93-100.

44. Bille J, Carstens K. Approximal caries progression in 13- to 15-year-old Danish children. Acta Odontol Scand 1989;47:347-354.

45. Holmen L, Thylstrup A, Ogaard B, Kragh F. A scanning electron microscopic study of 
progressive stages of enamel caries in vivo. Caries Res 1985;19:355-67.

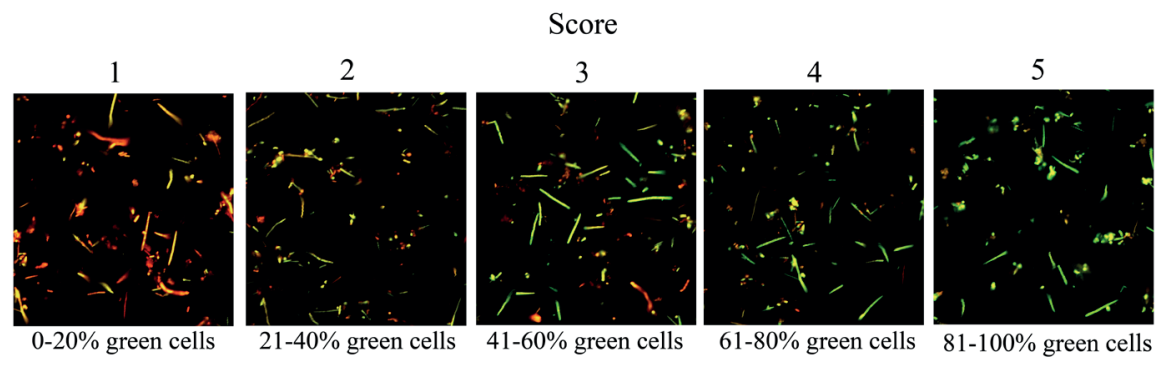

Figure 1. Images representing the different scores. Acid tolerant bacteria appear green and nonacid tolerant bacteria red when being stained with LIVE/DEAD® BacLight ${ }^{\mathrm{TM}}$ Viability stain.

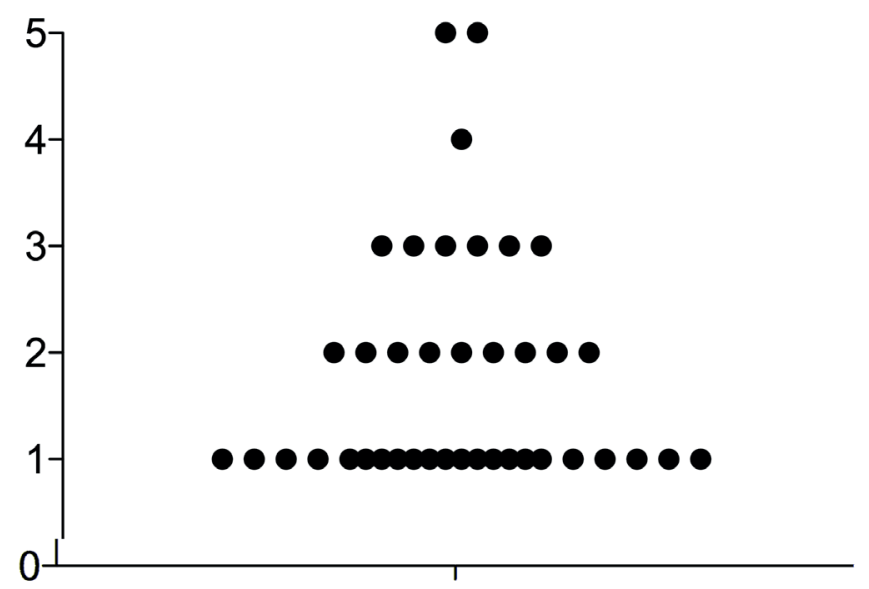

Figure 2. Distribution of acid tolerance scores at baseline. Each dot represents one individual in the study. 


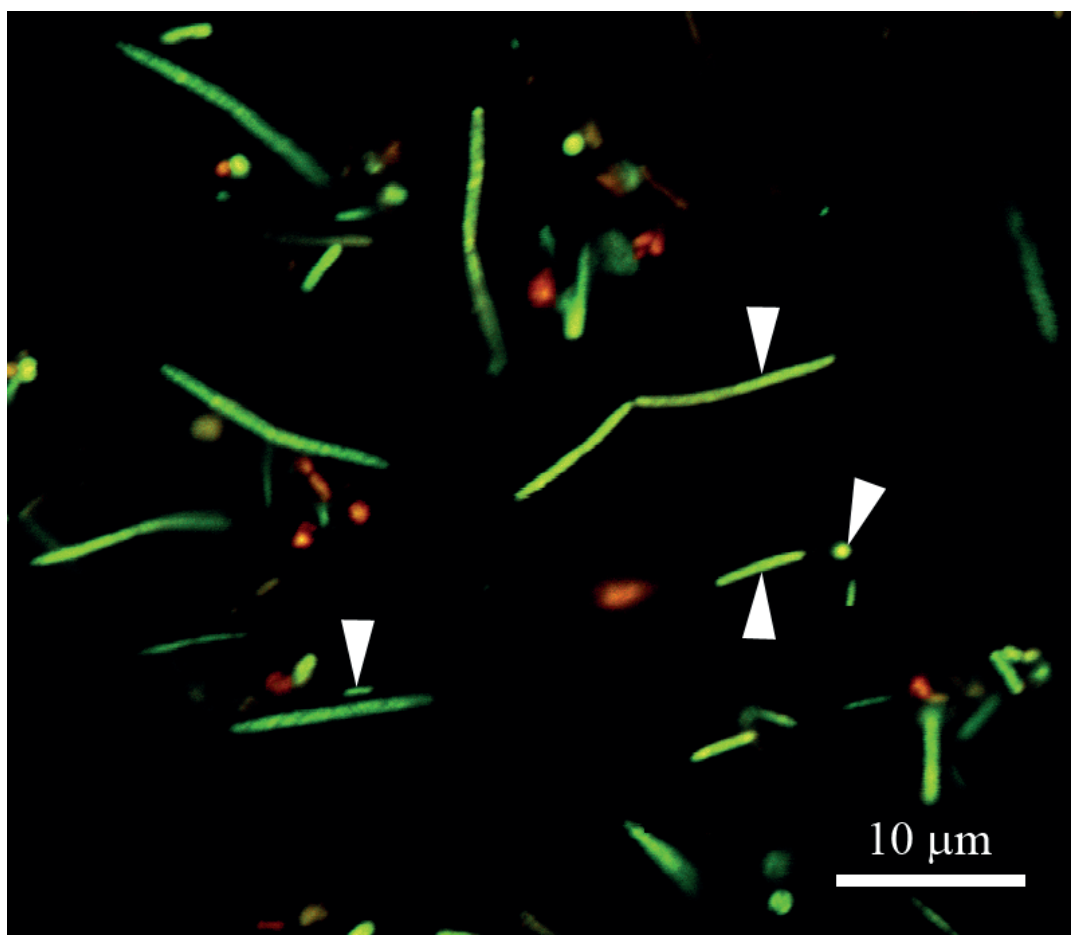

Figure 3. Image of biofilm sample showing acid tolerant bacteria [green cells] with different morphologies. 


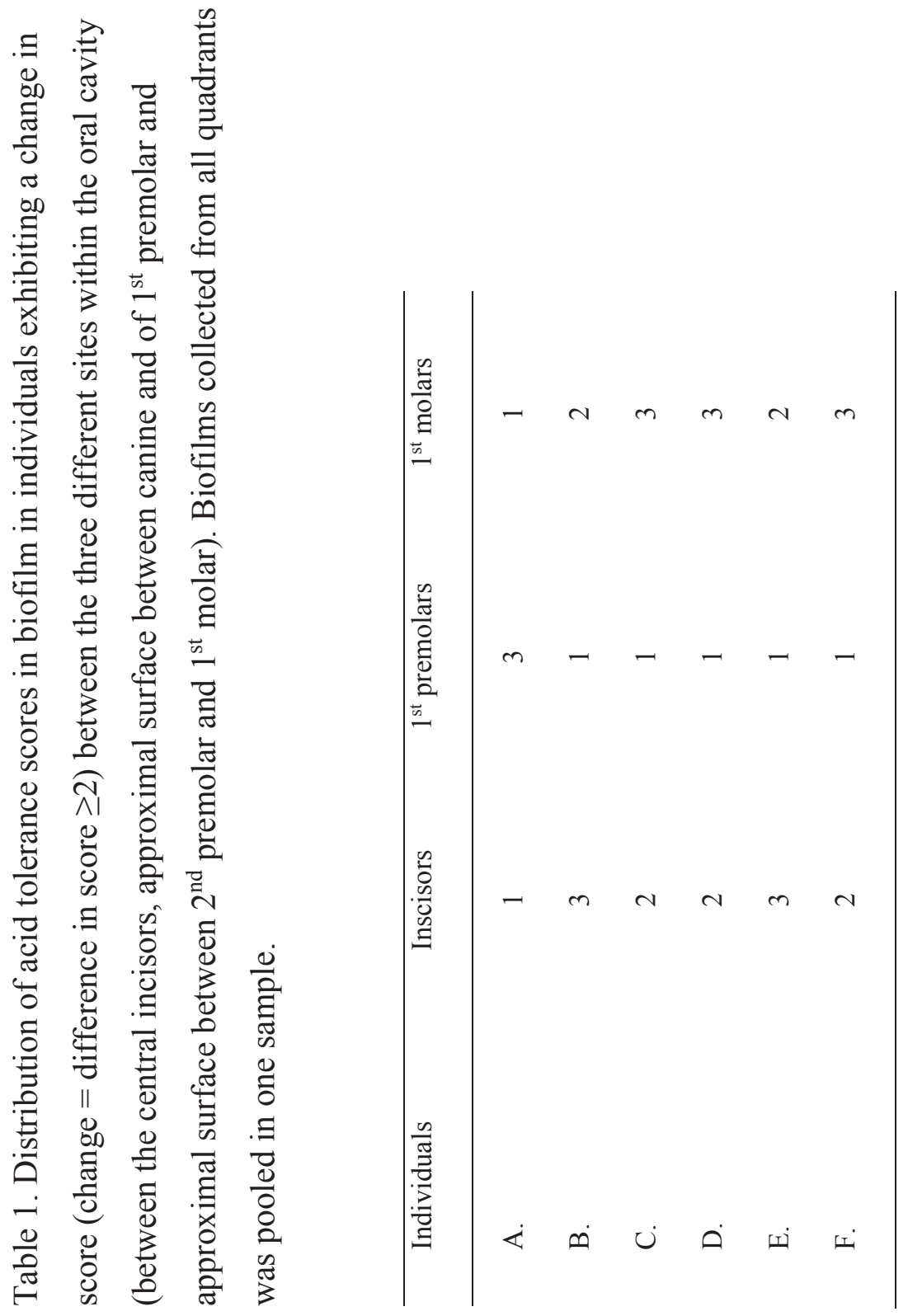




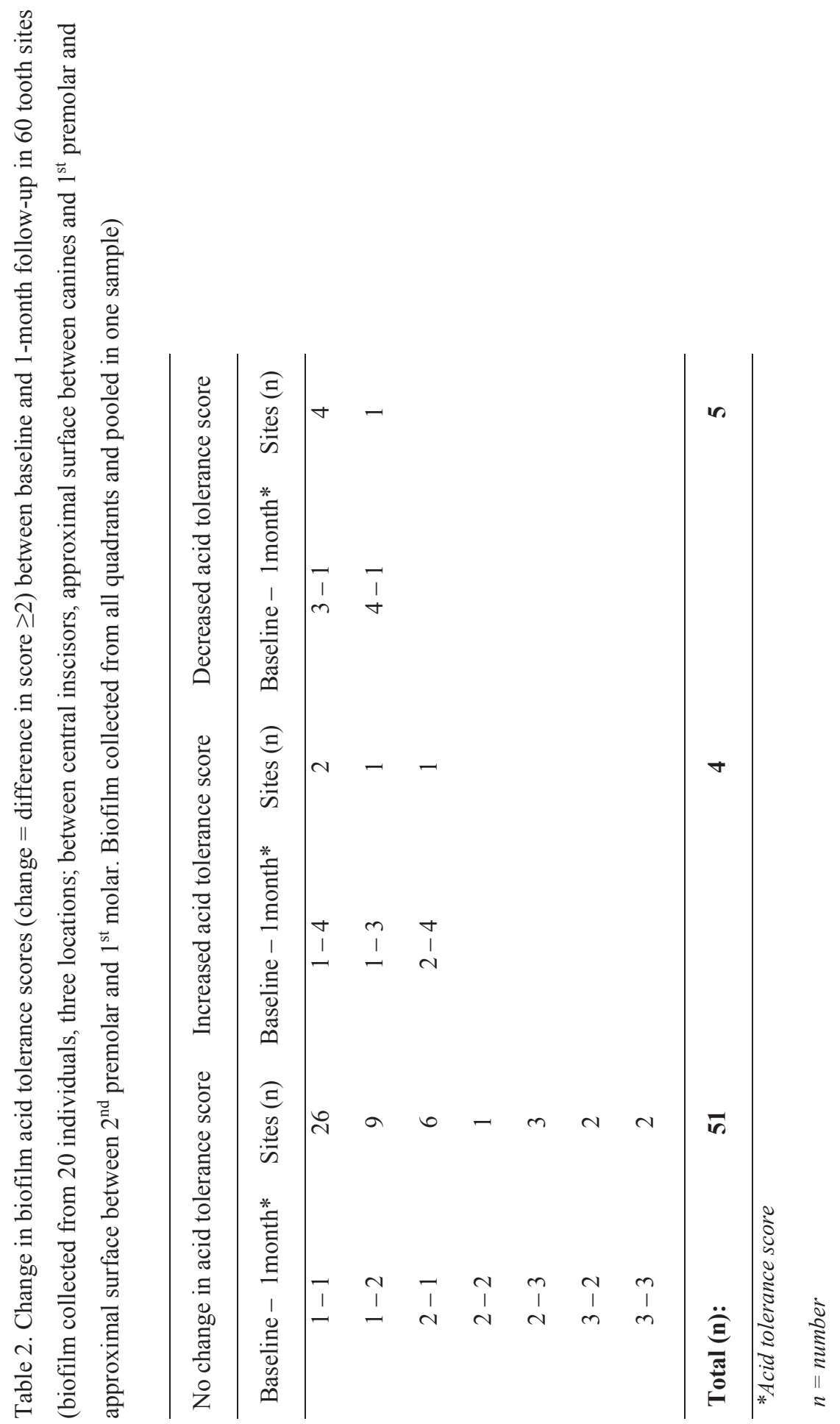


Table 3. Distribution of acid tolerance scores in biofilm collected from approximal surfaces between $2^{\text {nd }}$ premolars and first molars from 20 individuals at baseline, 6-months and 1-year follow-up

\begin{tabular}{|c|c|c|c|}
\hline Individuals & Baseline & 6 months & 1 year \\
\hline 1. & 1 & 1 & 1 \\
\hline 2. & 1 & 1 & 1 \\
\hline 3. & 1 & 1 & 1 \\
\hline 4. & 1 & 1 & 2 \\
\hline 5. & 1 & 2 & 1 \\
\hline 6. & 1 & 2 & 1 \\
\hline 7. & 1 & 3 & 1 \\
\hline 8. & 1 & 3 & 2 \\
\hline 9. & 1 & 3 & 3 \\
\hline 10. & 1 & 5 & 4 \\
\hline 11. & 2 & 1 & 2 \\
\hline 12. & 2 & 1 & 2 \\
\hline 13. & 2 & 2 & 2 \\
\hline 14. & 2 & 3 & 1 \\
\hline 15. & 3 & 1 & 2 \\
\hline 16. & 3 & 2 & 1 \\
\hline 17. & 3 & 2 & 2 \\
\hline 18. & 3 & 2 & 4 \\
\hline 19. & 5 & 1 & 1 \\
\hline 20. & 5 & 1 & 1 \\
\hline
\end{tabular}




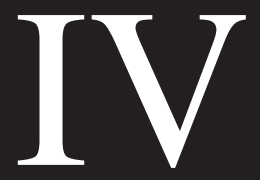





\section{Threshold Values Affect Diagnostic Accuracy of Caries Risk Assessment Methods}

Anna Senneby, Jessica Neilands, Gunnel Svensäter, Björn Axtelius, Madeleine Rohlin

Faculty of Odontology, Malmö University, 20506 Malmö, Sweden

Short title: Methods for Caries Risk Assessment

Correspondence to:

Anna Senneby

Malmö University, Faculty of Odontology, SE-205 06 Malmö, Sweden

Phone number: +46 734298939

E-mail: anna.senneby@mah.se

3 figures, 3 tables and 6 Appendices

Declaration of interests: No conflict of interests 


\section{ABSTRACT}

Objective: To evaluate diagnostic accuracy of methods for caries risk assessment and examine the effect of threshold values on accuracy estimates.

Material and Methods: Adolescents, aged 12, visiting two clinics, were examined by visual/tactile examination and bitewing radiography initially and after one year. Two established methods, previous caries experience and dentists' risk assessments and one candidate method, acid tolerance of dental biofilm, were applied. Reference standard comprised coronal caries lesion progression at one year. Diagnostic accuracy was calculated for different pre-specified thresholds of the methods and reference standard.

Results: Caries lesion progression occurred mainly within enamel approximally. Accuracy estimates changed with different threshold values of the index tests and of the reference standard. Patient spectrum differed between the two clinics, which resulted in different accuracy estimates for the samples of the clinics. Generally, negative predictive values were high, whilst positive ones were low indicating that it is more efficient in finding individuals, who are at low risk of developing caries lesions than those with increased risk.. Most positive likelihood ratios for the methods were $<5$ indicating small increases in the post-test probabilities.

Conclusions: As thresholds and patient spectrum affected diagnostic accuracy it may be difficult to design a universal model for caries risk assessment. Foremost, a model should take clinical questions and decisions that have to be made, based on the risk assessment in the actual setting into consideration.

Key words: Acid tolerance, Caries increment, Likelihood ratios, Microbiology, Predictive values 


\section{INTRODUCTION}

In children and adolescents, caries is one of the most prevalent diseases, though the distribution and severity vary within the same region or country [1]. Hence, there is a skewed distribution of caries disease. Therefore, today's challenge is to identify the individuals at an increased risk of developing caries lesions so that interventions can be tailor-made to them as well as to those not at risk. BY doing this the number of redundant examinations and interventions can be reduced. Thus there is a necessity to identify accurate methods, enabling clinicians to identify individuals at increased risk of caries lesion development.

Contemporary methods used to identify individuals at increased risk of caries lesion development include previous caries experience, tests using genotypes of microbiota, salivary buffering capacity, salivary flow rate, dental plaque amount, dietary habits and sociodemographic/economic variables. The accuracy of these methods, as suggested by the literature, varies considerably. Previous caries experience is considered the most accurate single predictor in school children and adolescents [2]. However, according to a recent systematic review [3], tests using salivary mutans streptococci present a higher diagnostic accuracy expressed as likelihood ratios than previous caries experience in children with primary teeth does. Accuracy estimates were calculated from data presented in a study on previous caries experience [4] and in another study on salivary mutans [5]. It was evident that, besides caries prevalence, different thresholds of the methods changed the diagnostic accuracy estimates. Evidence regarding methods other than previous caries experience and salivary mutans streptococci is lacking [3]. Moreover, systematic reviews on methods used for caries risk assessment have concluded that the quality of studies are uniformly poor due to a lack of clear reporting and that there is a need for validation studies $[2,3,6]$. Previous caries experience and dentists' risk assessments are particularly interesting to analyse, as these methods are feasible and often used in dental practice.

Studies on caries risk assessment of microbiological methods using genotypes, such as mutans streptococci or lactobacilli from saliva, show that the proportion of false negative diagnoses are high [5, 7-11]. Hence, the lack of numerous mutans streptococci does not exclude the possibility of an acid tolerant microflora and an increased risk for caries development. This indicates that other genotypes and/or phenotypic expressions of mutans streptococci are involved in the demineralisation process. At the same time, numerous false positives suggest that some individuals seem to harbour large numbers of mutans streptococci in saliva [5] or in plaque [12] without increased risk of developing caries lesions. This may support the ecological plaque hypothesis, whereby the proportion of acid tolerant microflora 
in dental biofilm, rather than the number of mutans streptococci or lactobacilli, is the underlying cause of caries lesions [13]. According to the ecological plaque hypothesis, presence of carbohydrates results in various acids as end products from microbiota and lower the $\mathrm{pH}$ in the biofilm. The effect is twofold; bacteria able to survive and metabolise at low pH-levels will have a competitive advantage compared to non-adapted bacteria. This selection and enrichment of acid tolerant and aciduric bacteria result in more acid being produced at even faster rates enhancing demineralisation further [13-15]. To the best of our knowledge, the proportion of acid tolerant bacteria in dental biofilm have not previously been used as a method to identify individuals at increased risk of caries lesion development.

The aim of this study was to estimate diagnostic accuracy of three methods (index tests) to identify adolescents with an increased risk of dental coronal caries lesion development: I. previous caries experience, II. dentists' risk assessments, and III. acid tolerance of dental biofilm. Special attention was paid to the effect of different threshold values of the index tests and of the reference standard on diagnostic accuracy estimates. 


\section{MATERIAL AND METHOD}

\section{In general}

In order to achieve an approach that was accurate, thus enabling the reader to assess potential bias, this prospective study was conducted in accordance with "Standards for Reporting of Diagnostic Accuracy", the so-called STARD-statement [16,17] and a modified STARDchecklist was applied (Appendix Table 1). In addition, careful attention was paid to items presented in QUADAS-2 "Quality Assessment of Diagnostic Accuracy Studies" [18] and GRRAS "Guidelines for Reporting Reliability and Agreement Studies" [19]. An overview of study characteristics is presented in Appendix Table 2.

Three methods, from now on referred to as index tests, which may be used to identify adolescents with increased risk of dental coronal caries lesion development, were performed initially during the same visit. We defined risk as the probability of an unwanted event, i.e. the coronal dental caries lesion for an adolescent. The index tests were: I. previous caries experience, II. dentists' risk assessments and III. acid tolerance of dental biofilm. The reference standard was based on the results of clinical and radiographic examinations performed after one year. Different thresholds for the index tests were pre-specified: index test I was based on thresholds used in clinical practice, index test II on a current risk assessment program of the public dental health service of one county, and thresholds of test III on scores used in an ongoing intervention study. Different thresholds were also applied for the reference standard, which comprised caries increment over one year.

\section{Sample}

All adolescents, aged 12, visiting two public dental clinics (clinic $\mathrm{A}$ and clinic $\mathrm{S}$ ) in two Swedish counties, were approached during their regular dental check-up appointment. The recruitment period started in October 2012 and ended in January 2015. Individuals fulfilling the inclusion criteria were consecutively enrolled. The inclusion criteria were as follows: healthy (no systemic disease or oral disease possibly affecting the oral cavity) adolescents with permanent posterior teeth, who together with their parents gave informed written consent to participate, and had received no antibiotic treatment during the last 3 months. The number of recruited adolescents in clinics $\mathrm{A}$ and $\mathrm{S}$ is presented in the flow-chart (Figure 1), designed and modified according to STARD [16].

Some adolescents were randomly allocated to an intervention group (Group I). In addition to their normal oral hygiene routines, the patients drank a dose of fluoride solution ( $0.75 \mathrm{mg}$ for children aged 12 years and $1.0 \mathrm{mg}$ for children $>13$ years) in $200 \mathrm{ml}$ of cow's 
milk on a daily basis. Patients in another group (Group C) consumed $200 \mathrm{ml}$ of milk without fluoride daily. The fluoride/placebo solution was supplied as individually packaged daily doses in $0.5 \mathrm{ml}$ sterile water.

Ethical approval was granted by the Regional Ethical Review Board, Lund, Sweden (diary number: OD62-2012/122).

\section{Index tests}

\section{Previous caries experience}

Previous caries experience was based on the visual and tactile examination of all teeth and on bitewing radiography of the posterior teeth. The visual and tactile examination was performed under lighting with a mirror and a sharp probe (clinic A: Top Dent Instr. Sond EXP 23-12, DAB Dental AB, Sweden; clinic S: 17-23 XSI Explorer, LM-Dental AB, Sweden) by one general dental practitioner (GDP) in each clinic. The GDPs' professional experience was 36 and 28 years, respectively. Teeth were air-dried before examination and each surface examined separately (incisal, mesial, buccal, distal, and lingual surfaces of incisors and cuspids; occlusal, buccal and lingual surfaces of premolars and molars). Surfaces were scored according to The International Caries Detection and Assessment System (ICDAS TM) (Ismail et al., 2007) with a score from 0 to 6 [20]. Results were recorded in pre-designed protocols devised for a clinical trial.

In the same session, one bitewing radiograph was exposed in each side of the mouth. The radiographic equipment, storage, export of images, and the quality control of the equipment and images have previously been described [21]. The radiographic assessment of the approximal surfaces of the posterior teeth (all visible mesial and distal surfaces) was performed by two oral and maxillofacial radiologists in consensus using Synedra ${ }^{\circledR}$ view personal (Planmeca, Finland) and a computer (HP Compaq Elite 8100 SFF, US). The room had dimmed light (ambient light below 20 lux) and the viewing distance to the screen was 30 $40 \mathrm{~cm}$. No image enhancement or altering of image characteristics was performed. Surfaces were assessed at baseline as sound, caries lesion in the outer enamel, caries lesion in the inner enamel (reaching the dento-enamel junction), and caries lesion in the dentine.

The pre-specified thresholds of previous caries experience were defined as: DMFS $\geq 1, \geq 2, \geq 3, \geq 4$ or $\geq 5$ (decay included all dental surfaces and caries lesion severities) Only fillings due to caries were included (i.e. fissure sealants, fillings due to fracture, disturbance in tooth surfaces such as mineralisation disturbances, were excluded).

Dentists' risk assessments 
The GDP in each clinic performed risk assessment on the basis of previous and present clinical and radiographic examinations of primary and permanent teeth. Since all adolescent had attended the clinics for two years or longer, it was possible to acquire the records and assess caries lesion progression during the previous two years.

The pre-specified thresholds for dentists' risk assessments were based on criteria applied in the public dental health service of one county and defined as:

- low risk $=$ no new enamel caries lesion during the previous two years

- high risk $=\geq 1$ dentinal caries lesion (arrested or progressed), or $\geq 1$ new approximal caries lesion in enamel during the previous two years.

Acid tolerance of dental biofilm

Dental biofilm was collected using the same method, at baseline examination and 6 months after baseline. For details regarding sampling and transport, laboratory method and method of assessment of acid tolerance, see Senneby et al. [22]. Oral biofilm was collected with a Quickstick (Dab Dental AB, Upplands Väsby, Sweden) from the mesial surfaces of all first molars and pooled, resulting in one sample per individual. Each sample was suspended in 200 $\mu l$ tryptone-yeast extract broth ( $\mathrm{pH} 3.5$ ) and incubated at $37^{\circ} \mathrm{C}$ for 2 hours. After incubation, microorganisms were stained using LIVE/DEAD ${ }^{\circledR}$ BacLight ${ }^{\mathrm{TM}}$ Fluorescent Stain (Molecular Probes, Eugene, USA) [23] and added to an Ibidi mini flow cell (IbidiR $\mu$-Slide, Ibidi GmbH, Martinsried, Germany). Each biofilm sample was examined using an inverted confocal scanning laser microscope (Nikon Eclipse TE2000, Nikon Corp., Tokyo, Japan). Live bacteria (acid tolerant) appear green, while dead (non-acid tolerant) bacteria appear red. Ten random images for each individual were manually recorded from the biofilm sample. All images were assessed by one rater, an oral biologist (experience $>10$ years), in a university environment, according to an interval scale presented in Appendix Figure 2 with 5 possible scores [24].

The pre-specified thresholds for acid tolerance of dental biolfilm were: score $\geq 1, \geq 2, \geq 3, \geq 4$ or 5

\section{Reference standard}

To determine the reference standard, from now on referred to as caries increment, the visual/tactile examination and bitewing radiography was repeated after one year by the same GDPs, who performed the initial examinations and who had access to records from baseline. In both clinics, the examinations were performed as described for index test "I. Previous caries experience". The records of the baseline examination and examination at one year were compared for occlusal/incisal, buccal and lingual surfaces of all teeth and of the approximal 
surfaces of incisors and cuspids regarding the ICDAS scores. An increase in ICDAS score over the year was interpreted as caries increment for these surfaces.

For approximal surfaces of premolars and molars, the bitewing images were displayed side-by side on a monitor: one image pair from baseline and one from the 1-year follow-up. Two radiologists in consensus performed the assessments without access to results of the clinical examinations. Figure 2 displays the system used for assessment of caries lesion progression of each approximal surface, a system based on the classification system designed to visualize sound, arrested, and progressed caries lesions [21].

The pre-specified thresholds for caries increment were based on thresholds used in clinical practice and defined as:

DMS $\geq 1, \geq 2$ or $\geq 3$ (decay included number of new or progressed caries lesions in all dental surfaces and lesion severities).

\section{Rater agreement}

Assessment of proportion acid tolerant cells

To establish inter- and intra-rater agreement of the assessments of proportion acid tolerant cells, seven raters, all experienced within the field (range 10-25 years), were asked to assess 50 images of samples, all originating from clinic A. The images represented biofilm samples taken from the mesial surface of the first molars and were selected to represent 10 images of each score. For inter-rater agreement, the images were presented to the raters in random order one by one. The raters independently assessed and scored each image with access to Figure 3. Data was collected using a pre-designed form by one of the authors (AS), who was present during the assessments. No calibration exercise or other preparation preceded the sessions. For intra-rater agreement, two of the raters repeated their assessments after 14-21 days under the same conditions. The order of image presentation differed from the first session. Raters were blinded towards previous results.

Assessment of approximal caries lesions in bitewing radiographs

Intra-rater agreement of caries detection and caries lesion progression in posterior bitewing radiographs was based on the assessments of all visible approximal surfaces by the two radiologists in consensus. The radiographs were re-assessed in the same setting after 2 weeks. The radiographs were randomly presented at both occasions. During the second viewing, raters were blinded towards the results of their previous assessments.

For the assessments of caries detection, radiographs of 10 adolescents with different status concerning caries lesions (50\% with caries lesions) were selected. Surfaces were 
assessed as sound, caries lesion in the outer enamel, in the inner enamel (reaching the dentoenamel junction), or in the dentine. For assessment of caries lesion progression, pairs of radiographs of 10 adolescents with different status concerning caries lesion progression $(50 \%$ with caries lesion progression) were selected. Caries lesion progression was assessed using a classification system shown in Figure 2.

Analysis

The analysis was performed with the support of a statistician. The calculations were made with the aid of STATA 10 (StataCorp, LP, USA). Kappa values with 95\% confidence intervals (CI) were generated for inter- (for acid tolerance test) and intra-rater agreement and the percentage of agreement was calculated. The extent to which raters agreed was evaluated by means of kappa. Kappa values, varying from -1 to 1 , were categorised according to the scale suggested by Landis and Koch [25]. According to this scale, values less than zero are considered as poor agreement, 0.00 to 0.20 as slight, 0.21 to 0.40 as fair, 0.41 to 0.60 as moderate, 0.61 to 0.80 as substantial, and values higher than 0.81 as almost perfect agreement.

\section{Flow and timing}

The recruitment process with regard to included individuals and withdrawals as well as individuals undergoing index test and reference standard is presented in Figure 1. Order of test execution and follow-up time interval is presented above under heading Material and Method. Home care recommendations and general guidelines regarding interventions for adolescents during the follow-up period, possibly affecting the condition, is summarized in Appendix Table 3. The GDPs assessed the need for interventions individually and their decision was based on the results of the clinical and radiographic examination at baseline. After 6 month and at the 1-year follow-up, adolescents and caregiver were asked about oral health care habits, general health condition, and medications.

\section{Calculation of diagnostic accuracy}

Diagnostic accuracy of the index tests, previous caries experience, dentists' risk assessments, and acid tolerance of dental biofilm, was calculated through construction of $2 \times 2$ tables crossclassifying number of true positive (TP), false positive (FP), false negative (FN) and true negative (TN) individuals. The sensitivity, specificity, predictive values and likelihood ratios were calculated, along with their corresponding $95 \%$ confidence intervals, for selected 
threshold values using the statistical software Meta-DiSc (version 1.4) [26]. For threshold values presenting zero individuals as TP and FN, diagnostic accuracy was not calculated. 


\section{RESULTS}

\section{Sample}

Figure 1 presents the number of adolescents recruited, withdrawals, and included for final analysis. In Clinic C, 104 adolescents were recruited and 29 withdrew, and the corresponding figures for Clinic S were 71 and 33, respectively. Analysis of two index tests at the 1-year follow-up was based on 113 adolescents and on acid tolerance test of 108 adolescents.

Table 1 presents included adolescents as well as those withdrawn with regard to caries prevalence at baseline and at the 1-year follow-up. There were no teeth missing due to caries. At baseline, caries prevalence of included adolescents (all surfaces and severities) was 39\% (DFS 1.7/DFT 1.5) and 63\% (DFS 2.8/DMFT 2.3) of withdrawals. In clinic A, DFS including enamel lesions of included adolescents was 2.0, whilst the corresponding DFS in clinic S was 1.4. In both clinics, DFS of withdrawals was higher than that of included adolescents (Table 1).

At the 1-year follow-up, caries prevalence was the same when expressed as percentage of adolescents in the clinics (45\%) but different when expressed as DFS (all surfaces and severities) and higher in clinic A (2.5) than in clinic S (1.6) (Table 1). At baseline and at follow-up, almost all caries lesions in clinic $\mathrm{S}$ and half of the lesions in clinic A were approximal (Table 1). A majority of the lesions were enamel lesions. Caries lesion progression occurred mainly as new enamel lesions or within the enamel of the approximal surfaces ( 86 and 78\%, respectively). In clinic A, nine adolescents received 17 new fillings during the follow-up, whilst no filling was performed in adolescents in clinic S.

In Table 2, the frequency distribution of adolescents with caries lesion progression at the 1-year follow-up and the index tests with different thresholds is presented. Caries lesion progression occurred in 25 (33\%) adolescents in clinic A and 8 (21\%) in clinic S. Four adolescents in clinic $\mathrm{A}$ and none in clinic $\mathrm{S}$ presented $\geq 3$ surfaces with caries lesion progression. For previous caries experience, $25 \%$ of adolescents in clinic A and $11 \%$ in clinic S presented the threshold $\geq 3$ caries lesions. Dentists assessed $20 \%$ in clinic A and $24 \%$ in clinic $\mathrm{S}$ as having a high risk of caries. Acid tolerance was scored $\leq 3$ for the majority of adolescents in both clinics (Table 2).

\section{Rater agreement}

Assessment of proportion acid tolerant cells 
All 50 selected images and all seven raters' assessments were included for final analysis. Inter-rater agreement for all scores resulted in moderate agreement (kappa 0.59; CI 0.496 0.691). Pair-wise inter-rater agreement was $50-86 \%$, corresponding to fair to substantial agreement (kappa 0.38-0.83), most values being moderate to substantial agreement.

Implementing thresholds for score 1 vs. 2, 3, 4, 5, 1, 2, vs. 3, 4, 5 or 1, 2, 3 vs. 4, 5, resulted in substantial agreement (kappa $\geq 0.76$ ) for all thresholds, though, threshold 1, 2, 3, 4 vs. 5 showed almost perfect agreement (kappa 0.89; CI 0.758 - 0.973). Intra-rater agreement varied between substantial and almost perfect for all thresholds, with no major difference between raters, being 74\% (kappa 0.67; CI 0.52 - 0.81) for both raters and all scores. The intra-rater agreement for all scores for the rater, who assessed samples included in the present study (author JN), was 94\% (kappa 0.92).

\section{Assessment of Approximal Caries Lesions in Bitewing Radiographs}

For the assessments of caries lesion detection and progression, all visible approximal surfaces in the selected posterior bitewing radiographs and all the assessments were included for final analysis. Intra-rater agreement for assessment of caries lesion detection regarding healthy $v s$. surface with caries lesion (any severity) was $96 \%$, unweighted kappa 0.63 (CI 0.43-0.82), corresponding to substantial agreement. Intra-rater agreement regarding different severities (healthy, caries lesion in the outer enamel, in the inner enamel or in the dentine) was $94 \%$ (weighted kappa 0.53, CI 0.34-0.71). Intra-rater agreement for assessment of caries lesion progression $v s$. no progression resulted in similar values, $96 \%$, unweighted kappa being 0.63 (CI 0.42-0.83), considered as substantial agreement.

\section{Accuracy estimates of the index tests}

In general

In Table 3, representative accuracy estimates for different threshold values of the index tests and of the reference standard are summarised. $\mathrm{LR}+\mathrm{s}$ for previous caries experience increased with an increase of the index test threshold given a reference standard threshold of DS $\geq 1$, in particular for the sample in clinic S. For the acid tolerance test, there was a similar increase for the sample in clinic $S$ when the index test threshold was increased from $\geq 3$ to $\geq 4$ given a reference standard of DS $\geq 1$ (Table 3). An increase of the reference standard threshold value from DS $\geq 1$ to DS $\geq 2$ resulted in a minor decrease of the LR+ and an increase of the negative predictive values for the three index tests. The fact that sample characteristics of the two clinics influenced the accuracy estimates is illustrated by the LR + for the index test previous caries experience threshold values $\geq 2$ and $\geq 3$, given the reference standard $\geq 1$ being higher for 
the sample in clinic $S$ than that in clinic A (Table 3). For acid tolerance test score $\geq 4$, LR+ was somewhat higher and LR- was lower for the sample in clinic S compared to clinic A (Table 3). Thus, the estimates of diagnostic accuracy were affected by the threshold values of (i) the index tests and (ii) the reference standard, as well as of (iii) the sample characteristics. Previous caries experience

Detailed data on accuracy of previous caries experience, with pre-specified thresholds and different thresholds for the reference standard (caries increment at one year), are presented in Appendix Table 3. For the sample of both clinics, sensitivity ranged between 0.30 and 0.86 and specificity between 0.50 and 0.95 . Also, for a sample within the same clinic, the range of the accuracy estimates was wide. Negative predictive values were generally high (range 0.72 1) being $\geq 0.90$ for many thresholds, i.e,. individuals not exhibiting any previous caries experience will be correctly identified as having no caries lesion progression. The low positive predictive values in general indicated that this test performs poorly when used to identify adolescents with increased risk of coronal caries lesion development. For the sample in clinic S, high LR+ (7.5) and low LR- (0.3) was achieved given the index test threshold of $\mathrm{DFS} \geq 2$ and reference standard DS $\geq 1$ (Table 3 ). Even higher $\mathrm{LR}+\mathrm{s}$ were achieved for the index test thresholds DFS $\geq 3$ and reference standard DS $\geq 1$ (Table 3 ). In clinic A, the highest LR+ (6.7) and lowest LR- (0.3) were obtained for the index test threshold DFS $\geq 4$ and reference standard DS $\geq 2$.

\section{Dentists' risk assessments}

Accuracy of dentists' risk assessments, versus pre-specified thresholds of the reference standard, is presented in Appendix Table 4. Sensitivity ranged between 0.33 and 0.75 and specificity between 0.77 and 0.84 . Most negative predictive values were around 0.90 , whilst only two positive predictive values were $\geq 0.50(0.50,0.57)$. In clinic A, the best LRs, LR+ 4.44 and LR- 0.30, were achieved for a high reference standard threshold (DS $\geq 3$ ), whilst in clinic $\mathrm{S}$ the highest $\mathrm{LR}+3$ was achieved for the lowest threshold ( $\mathrm{DS} \geq 1$ ).

\section{Acid tolerance of dental biofilm}

Accuracy of acid tolerance test with pre-specified thresholds, versus thresholds for the reference standard, is presented in Appendix Table 5. For the acid tolerance test, sensitivity was low ranging between 0.04 and 0.75 , whilst specificity was somewhat higher ranging between 0.47 and 0.96 , in particular for score $\geq 4$. Most negative predictive values were high, in particular for the sample in clinic S, whilst positive predictive values were low. For the sample in clinic S, more favourable LRs were achieved than for the sample in clinic $\mathrm{A}$, in particular for score $\geq 4$ given reference standard DS $\geq 1$ (LR+6.2; LR- 0.17) (Table 3). 


\section{DISCUSSION}

\section{Methodological considerations}

Deficiencies in the reporting of methods on diagnostic accuracy have been identified in increasing number of studies [27] as has potential sources of bias [28, 29]. Thus, striving towards transparent and complete reporting, enabling validation and critical appraisal, the STARD-statement [16] and relevant items originating from the QUADAS-2 protocol [18] were implemented. With the limited sample size and low number of adolescents developing caries lesions, it is undoubtedly crucial to focus on methodological quality. Even if the rate of lesion progression is at the highest from the ages of 12 to 16 [30], a 1-year follow-up period is a rather short time period. The sample size may still be acceptable, since results of limited samples could ultimately be combined in systematic reviews and meta-analysis, and thereby contribute to the collective body of evidence [31]. The access to bitewing radiography in this study enhanced the study quality as bitewing radiography adds to the diagnostic yield of visual and tactile examinations $[32,33]$. Following the philosophy of interceptive prevention, the inclusion of enamel caries in the diagnostic criteria is important. Interventional strategies have changed with efforts to arrest lesions within the enamel and to reduce the use of operative treatment for cavitated lesions. As the tendency to postpone operative treatment has continued over the years [34], it is even more important to monitor caries lesion progression before cavitation. In the present study, most caries lesions were enamel lesions. Moreover, the highest number of surfaces with caries lesion progression was approximal ones, which may be difficult to assess without bitewing radiography. It may be reasonable to infer that bitewing radiographs are crucial in studies of caries risk assessment as making a correct diagnosis is a prerequisite for appropriate management [35]. Nevertheless, bitewing radiography was applied only in a few studies of methods on caries risk assessment [3], and in studies including bitewing radiography lesions progression was not assessed by comparing bitewing images over time simultaneously, a common approach in clinical practice.

Eligible adolescents in the catchment regions of the two clinics were not described. The fact that in the catchment area of clinic A there are many adolescents who have recently arrived in Sweden and who are not yet registered with the public dental health system, made any description of an eligible sample difficult. These adolescents would probably not resemble the studied samples. This was the case for withdrawals, who presented higher caries prevalence (63\%) and DFS/DFT (2.8/2.3) at baseline compared to included adolescents (39\%; 1.7/1.5). Thus, a cross section of the target population was not achieved, which contributed to the estimated accuracy of methods being underestimated when compared to the ideal 
circumstances.

The frequencies of pre-specified threshold for the index tests and for the reference standard were presented to facilitate the generation of $2 \times 2$ tables and other calculations of diagnostic accuracy in future studies. With the same intention, diagnostic accuracy was presented as several estimates. Although LRs are considered to be more clinically meaningful than other measures [36], these estimates are probably the least well understood ones. This may reflect a lack of familiarity with these measures rather than suggesting that they are less comprehensible [37]. LRs state how many times more likely particular test results are in patients with disease than in those without disease. They have the advantage of incorporating all four cells of a 2 × 2 table in contrast to sensitivity, specificity and predictive values, which makes use of only two cells $[39,39]$. Therefore, we prioritised LRs when summarizing most outcomes of the index tests.

\section{Discussion of results}

We showed that estimates of diagnostic accuracy were affected by the thresholds of the index tests as shown for medical tests [36]. For caries lesion detection in bitewing radiography, accuracy estimates for the threshold dentine lesions increased compared to any kind of proximal lesions [40]. As far as we know this has previously not been emphasized in studies on caries risk assessment. In most studies, the index test thresholds dmfs or DMFS $>0$ were applied for previous experience, whilst different thresholds, but only one in each study, were studied for microbiota tests [3]. Data on samples of adolescents applying different thresholds for predicting caries increment were, however, presented for two index tests: caries experience [4] and salivary levels of mutans streptococci and lactobacilli [5]. From these data it can be calculated that different thresholds of the index tests influence accuracy estimates given the same reference standard of a sample, this is in line with our results. As the index test thresholds influence accuracy estimates, it is vital that a frequency distribution for several prespecified thresholds of the index tests and of caries increment is presented in future studies of caries risk assessment. This will facilitate comparisons of results from different studies and make meta-analyses possible.

According to reviews of diagnostic test accuracy studies in medicine, there is consistent evidence that test accuracy may vary in different clinical populations and depending on disease prevalence $[28,41]$. With a low prevalence, e.g. in screening, there may be more individuals in whom the condition is less severe and fewer individuals in whom the 
condition is more severe. In the context of caries risk assessment, it has been highlighted in a review that "the predictive validity of caries risk-assessment strategies depended strongly on the caries prevalence and characteristics of a population" [42]. Thus, as displayed by our results, accuracy estimates are "true" only under specific conditions. Although caries prevalence expressed as percentage of individuals with caries of the two clinics was similar, accuracy estimates were different given the same thresholds of the index tests and the reference standard. The characteristics of the two samples were different when it comes to mean DFS at baseline and at follow-up, as well as lesion severity and number of surfaces with caries lesion progression. This calls for a detailed description of the caries status in future studies to make sub-grouping on caries risk assessment possible.

The choice of the reference standard of thresholds also influenced the accuracy estimates, although to a minor degree. According to the systematic review by Senneby et al. [3], only one study [4] applied bitewing radiography together with several reference standard thresholds. There are differences between the studies by David et al. [4] and the present one in follow-up time (6years vs. 1 year), index test thresholds (based on DFS of premolars and second molars $v s$. DFS of all teeth), and reference standard thresholds (based on dentinal lesions vs. lesions in enamel and dentine). Yet, the tendency in both studies was that sensitivity increased and specificity decreased for previous caries experience with increased reference standard threshold. Thus, the method performs better at identifying individuals with increased risk for developing 2 or more caries lesions.

In studies of caries risk assessment, risk is usually not defined although thresholds are set, but not pre-specified [3]. In this study, we defined risk as the probability of an undesirable (negative) outcome/event. Individual risk is the probability that an individual will experience an undesirable outcome [43]. However, when evaluating methods used to identify individuals at an increased risk of caries development, we base the accuracy estimates on the experience of a group. When considering these limitations on implementing described group characteristics in open-ended systems in conjunction with a non-communicable disease, such as caries, it may be more valid to focus on a lower abstraction level where the caries lesion progression is found, i.e. the actual site. The execution of thresholds based on number of sites does take this into account. The thresholds for new caries lesions, which were set to ds or DS $>0$ lesions in most studies [3], can be disputed. Is the progression of one caries lesion within the enamel over a year a high risk? The answer is dependent on the clinical decision following the prediction. Caries risk assessment is based on the assumption that individuals with caries increment receive care aimed at preventing future caries lesions [44]. However, according to a 
recent study "the caries risk assessment process was not accompanied by a corresponding targeted individual preventive care" as the high-risk group received less additional preventive measures than low-risk groups did [45].

The low level of caries prevalence and caries increment made it difficult to demonstrate high diagnostic accuracy. Sensitivity was generally lower than the corresponding specificity for the index tests. When prevalence is low, overall accuracy generally more closely resembles specificity; where prevalence is high; overall accuracy more closely resembles sensitivity [41]. Few accuracy estimates expressed as LR+ were higher than 5, which are deemed to provide a moderate increase in the post-test probability of disease [38]. The results of the present study indicated that $L R+$ decreased for the three index tests with increased reference standard threshold.

Previous caries experience is considered to be an important predictor [2, 44]. Accuracy estimates were somewhat better when compared to those of dentists' risk assessments and the acid tolerance test. In this study, sensitivity ranged between 0.21 and 0.94 and specificity between 0.20 and 1 , and the confidence intervals, i.e. the uncertainties surrounding the results, were wide ranging. The fact that the negative predictive value was close to 1 or 1 indicated that previous caries experience is an effective tool with which to screen out patients, who are not at an increased risk of new caries lesions. This is in line with results of previous studies 47, 48]. Dentists' risk assessments were based on a definition applied in the public dental service of one county. Thus, GDPs were not free to score according to their "clinical feeling". The definition was rather similar to previous caries experience with a threshold $\geq 1$ DS. Accuracy estimates expressed as LRs were comparable with those for previous caries experience. There was a difference between the LRs for the two samples, as LR+ increased and LR- decreased with an increase of the reference standard threshold in one clinic, and the opposite occurred in the other clinic with an decrease of LR+ with an increase of the reference standard threshold.

Acid tolerance test is based on that caries is a biofilm-mediated disease and the aetiology has, in recent years, been ascribed to the ecological plaque hypothesis. This explains the development of disease as the result of a shift towards a more acid tolerant microbiota [13]. In the present study, most individuals presented low scores of biofilm acid tolerance. In parallel with previous caries experience and dentists' risk assessments, the acid tolerance test was more efficient at finding individuals, who are at a low risk of developing caries lesions than at finding those with an increased risk. Caries lesions of all teeth surfaces were recorded, whilst biofilm sampling was restricted to the mesial surfaces of the first molars. This could be 
a limitation since the biofilm at the approximal space might not represent the biofilm at e.g. occlusal sites and thereby give false negative results. Studies with a larger population and extended follow-up time, as disease development often occur over a prolonged period of time, are needed to fully evaluate the acid tolerance as a test for caries risk assessment.

\section{Conclusions}

Negative predictive values of the methods were generally high indicating that it is more efficient at identifying individuals at low risk of developing caries lesions than those with increased risk. As most LR+s was below 5 there will be small increases in the post-test probability of caries lesions progression using the methods. Diagnostic test accuracy varies with threshold values of the index tests and of the reference standard. Furthermore, accuracy estimates vary with clinical variability as referred to as different caries status and caries lesion progression. Therefore, it may difficult to design a universal model for caries risk assessment. Foremost, a model should take clinical questions and decisions that have to be made, based on the risk assessment in the actual setting into consideration 
ACKNOWLEDGEMENTS 


\section{REFERENCES}

1. Petersen PE. Oral health. In: Heggenhougen K, Quah S, editors. International encyclopedia of public health, Vol. 4. San Diego: Academic Press; 2008. p. 677-85.

2. Mejàre I, Axelsson S, Dahlén G, Espelid I, Norlund A, Tranæus S, et al. Caries risk assessment. A systematic review. Acta Odontol Scand 2014;72:81-91.

3. Senneby A, Mejàre I, Sahlin NE, Svensäter G, Rohlin M. Diagnostic accuracy of different caries risk assessment methods. A systematic review. J Dent 2015;43:1385-93.

4. David J, Raadal M, Wang NJ, Strand GV. Caries increment and prediction from 12 to 18 years of age: a follow-up study. Eur Arch Paediatr Dent 2006;7:31-7.

5. Kingman A, Little W, Gomez I, Heifetz SB, Driscoll WS, Sheats R, et al. Salivary levels of Streptococcus mutans and lactobacilli and dental caries experiences in a US adolescent population. Community Dent Oral Epidemiol 1988;16:98-103.

6. Tellez M, Gomez J, Pretty I, Ellwood R, Ismail AI. Evidence on existing caries risk assessment systems: are they predictive of future caries? Community Dent Oral Epidemiol 2013;41:67-78.

7. Bankel M, Robertson A, Köhler B. Carious lesions and caries risk predictors in a group of Swedish children 2 to 3 years of age: one year observation. Eur J Paediatr Dent 2011;12:2159.

8. Petti S, Hausen HW. Caries prediction by multiple salivary mutans streptococcal counts in caries-free children with different levels of fluoride exposure, oral hygiene and sucrose intake. Caries Res 2000;34:380-7.

9. Grindefjord M, Dahllöf G, Modéer T, Caries development in children from 2.5 to 3.5 years of age: a longitudinal study. Caries Res 1995;29:449-54.

10. Demers M, Brodeur JM, Mouton C, Simard PL, Trahan L, Veilleux G. A multivariate model to predict caries increment in Montreal children aged 5 years. Commun Dent Health 1992;9:273-81.

11. Sullivan A, Schröder U. Systematic analysis of gingival state and salivary variables as predictors of caries from 5 to 7 years of age. Scand J Dent Res 1989;97:25-32.

12. Meurman PK, Pienihäkkinen K. Factors associated with caries increment: a longitudinal study from 18 months to 5 years of age. Caries Res 2010;44:519-24.

13. Marsh PD. Are dental diseases examples of ecological catastrophes? Microbiology 2003;149:279-94.

14. Marsh PD. Microbial ecology of dental plaque and its significance in health and disease. Adv Dent Res 1994;8:263-71. 
15. Bradshaw DJ, McKee AS, Marsh PD. Effects of carbohydrate pulses and $\mathrm{pH}$ on population shifts within oral microbial communities in vitro. J Dent Res 1989;68:1298-302. 16. Bossuyt PM, Reitsma JB, Bruns DE, Gatsonis CA, Glasziou PP, Irwig L, et al. STARD 2015: An updated list of essential items for reporting diagnostic accuracy studies. Clin Chem 2015;61:1446-52.

17. Bossuyt PM, Reitsma JB, Bruns DE, Gatsonis CA, Glasziou PP, Irwig LM, et al.: Standards for reporting of diagnostic accuracy: Towards complete and accurate reporting of studies of diagnostic accuracy: the STARD initiative. Standards for Reporting of Diagnostic Accuracy. Clin Chem 2003;49:1-6.

18. Whiting PF, Rutjes AW, Westwood ME, Mallett S, Deeks JJ, Reitsma JB, et al. QUADAS-2: a revised tool for the quality assessment of diagnostic accuracy studies. Ann Intern Med 2011;155:529-36.

19. Kottner J, Audige L, Brorson S, Donner A, Gajewski BJ, Hróbjartsson A, et al. Guidelines for Reporting Reliability and Agreement Studies (GRRAS) were proposed. Int J Nurs Stud 2011;48:661-71.

20. Ismail AI, Sohn W, Tellez M, Amaya A, Sen A, Hasson H, et al. The International Caries Detection and Assessment System (ICDAS): an integrated system for measuring dental caries. Community Dent Oral Epidemiol 2007;35:170-8.

21. Senneby A, Elfvin M, Stebring-Franzon C, Rohlin M. A novel classification system for assessment of approximal caries lesion progression in bitewing radiographs. Dentomaxillofac Radiol 2016;45:20160039. doi: 10.1259/dmfr.20160039.

22. Senneby A, Davies JR, Svensäter G, Neilands J. Acid tolerance of dental biofilms. Submitted to BMC Microbiology 2017.

23. Welin-Neilands J, Svensäter G. Acid tolerance of biofilm cells of Streptococcus mutans. Appl Environ Microbiol 2007;73:5633-8.

24. Neilands J, Petersson LG, Beighton D, Svensäter G. Fluoride-supplemented milk inhibits acid tolerance in root caries biofilms. Caries Res 2012;46:156-60.

25. Landis JR, Koch GG. The measurement of observer agreement for categorical data. Biometrics 1977; 33: 159-174.

26. Zamora J, Abraira V, Muriel A, Khan K, Coomarasamy A. Meta-DiSc: a software for meta-analysis of test accuracy data. BMC Medical Research Methodology 2006;12:31-43. ftp://ftp.hrc.es/pub/programas/metadisc/Metadisc_update.htm. MetaDiSc version 1.4 (Accessed April 26 ${ }^{\text {th }}, 2017$ ). 
27. Korevaar DA, Cohen JF, Hooft L, Bossuyt PM. Literature survey of high-impact journals revealed reporting weaknesses in abstracts of diagnostic accuracy studies. J Clin Epidemiol 2015;68:708-15.

28. Whiting PF, Rutjes AW, Westwood ME, Mallett S; QUADAS-2 Steering Group. A systematic review classifies sources of bias and variation in diagnostic test accuracy studies. $\mathrm{J}$ Clin Epidemiol 2013;66:1093-104.

29. Lijmer JG, Mol BW, Heisterkamp S, Bonsel GJ, Prins MH, van der Meulen JH, et al. Empirical evidence of design-related bias in studies of diagnostic tests. JAMA 1999;282:1061-6.

30. Mejàre I, Stenlund H, Zelezny-Holmlund C. Caries incidence and lesion progression from adolescence to young adulthood: a prospective 15-yearcohort study in Sweden. Caries Res 2004;38:130-41.

31. Guyatt GH, Mills EJ, Elbourne D. In the era of systematic reviews, does the size of an individual trial still matter? PLoS Med 2008;5:e4. doi: 10.1371/journal.pmed.0050004.

32. Wenzel A. Radiographic display of carious lesions and cavitation in approximal surfaces: advantages and drawbacks of conventional and advanced modalities. Acta Odontol Scand 2014;72:251-64.

33. Bader J, Shugars D, Bonito A. Systematic reviews of selected dental caries diagnostic and management methods. J Dent Educ 2001;65:960-8.

34. Vidnes-Kopperud S, Tveit AB, Espelid I. Changes in the treatment concept for approximal caries from 1983 to 2009 in Norway. Caries Res 2011;45:113-20.

35. Kostopoulou O, Oudhoff J, Nath R, Delaney BC, Munro CW, Harries C, et al. Predictors of diagnostic accuracy and safe management in difficult diagnostic problems in family medicine. Med Decis Making 2008;28:668-80.

36. Grimes DA, Schultz KF. Refining clinical diagnosis with likelihood ratios. Lancet 2005;365:1500-5.

37. Whiting PF, Davenport C, Jameson C, Burke M, Sterne JA, Hyde C, et al. How well do health professionals interpret diagnostic information? A systematic review. BMJ Open 2015;5:e08155.

38. Deeks JJ. Systematic reviews of evaluations of diagnostic and screening tests. BMJ 2001;323:157-62.

39. Weinstein MC, Fineberg HV, Elstein AS, Frazier HS, Neuhauser D, Neutra RR, et al. Clinical decision analysis. Philadelphia: WB Saunders Company; 1980. 
40. Schwendicke F, Tzschoppe M, Paris S. Radiographic caries detection: A systematic review and meta-analysis. J Dent 2015;43:924-33.

41. Leeflang MM, Bossuyt PM, Irwig L. Diagnostic test accuracy may vary with prevalence: implications for evidence-based diagnosis. J Clin Epidemiol 2009;62:5-12.

42. Zero D, Fontana M, Lennon AM. Clinical applications and outcomes of using indicators of risk in caries management. J Dent Educ 2001;65:1126-32.

43. Stern RH. Individual risk. J Clin Hypertens (Greenwich) 2012;14:261-4.

44. Batchelor PA, Sheiham A. The distribution of burden of dental caries in schoolchildren: a critique of the high-risk caries prevention strategy for populations. BMC Oral Health 2006;6:3.

45. Hänsel Petersson G, Ericson E, Twetman S. Preventive care delivered within Public Dental Service after caries risk assessment of young adults. Int J Dent Hyg 2016;14:215-9.

46. Caries - diagnosis, risk assessment and non-invasive treatment. A systematic review. Summary and Conclusions in English. Stockholm: Swedish Agency for Health Technology Assessment and Assessment of Social Services (SBU). Report no 188,2007.

http://www.sbu.se/globalassets/publikationer/content1/1/caries_summary_2008.pdf (Accessed April 26 2017). 


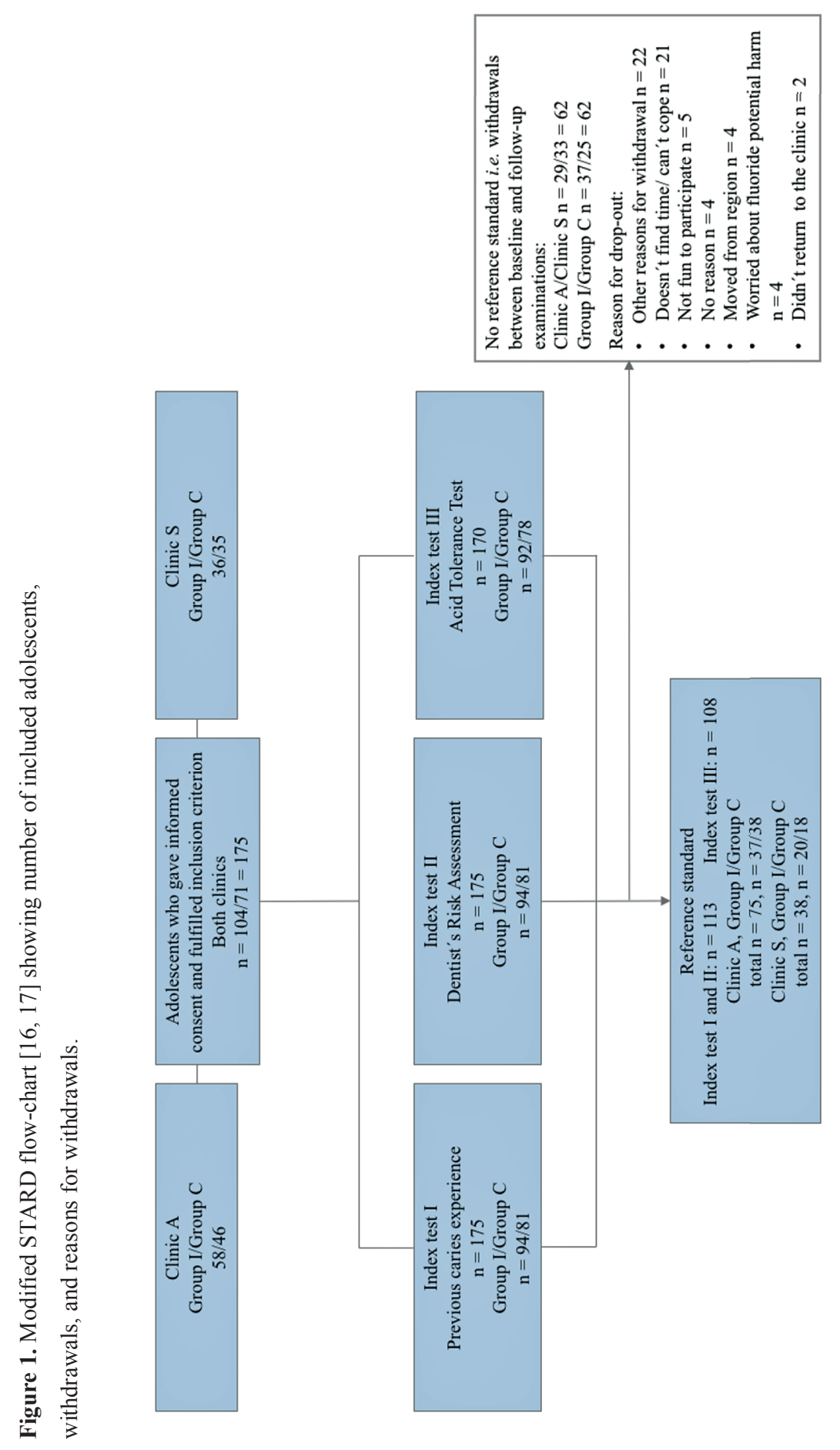




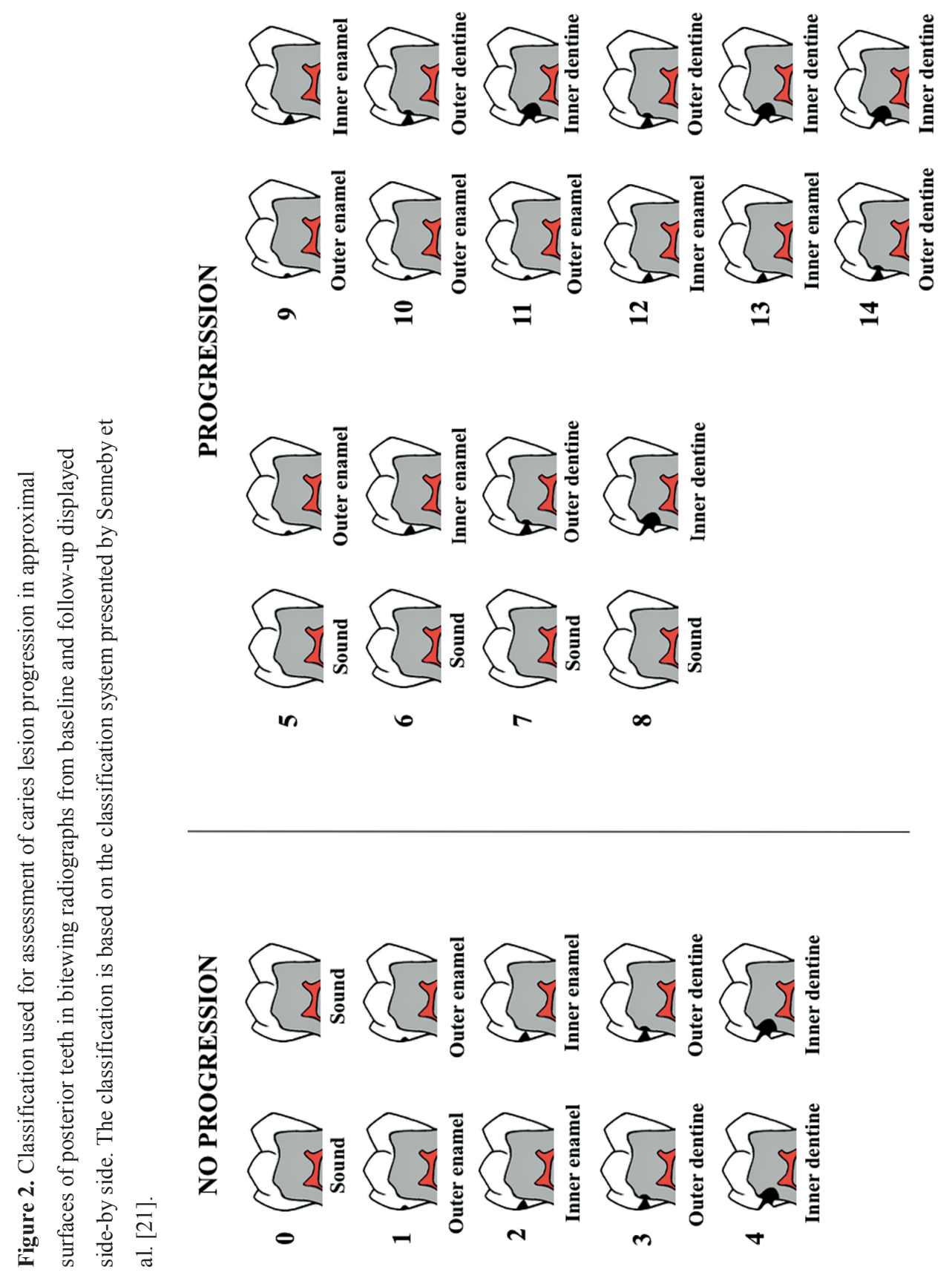




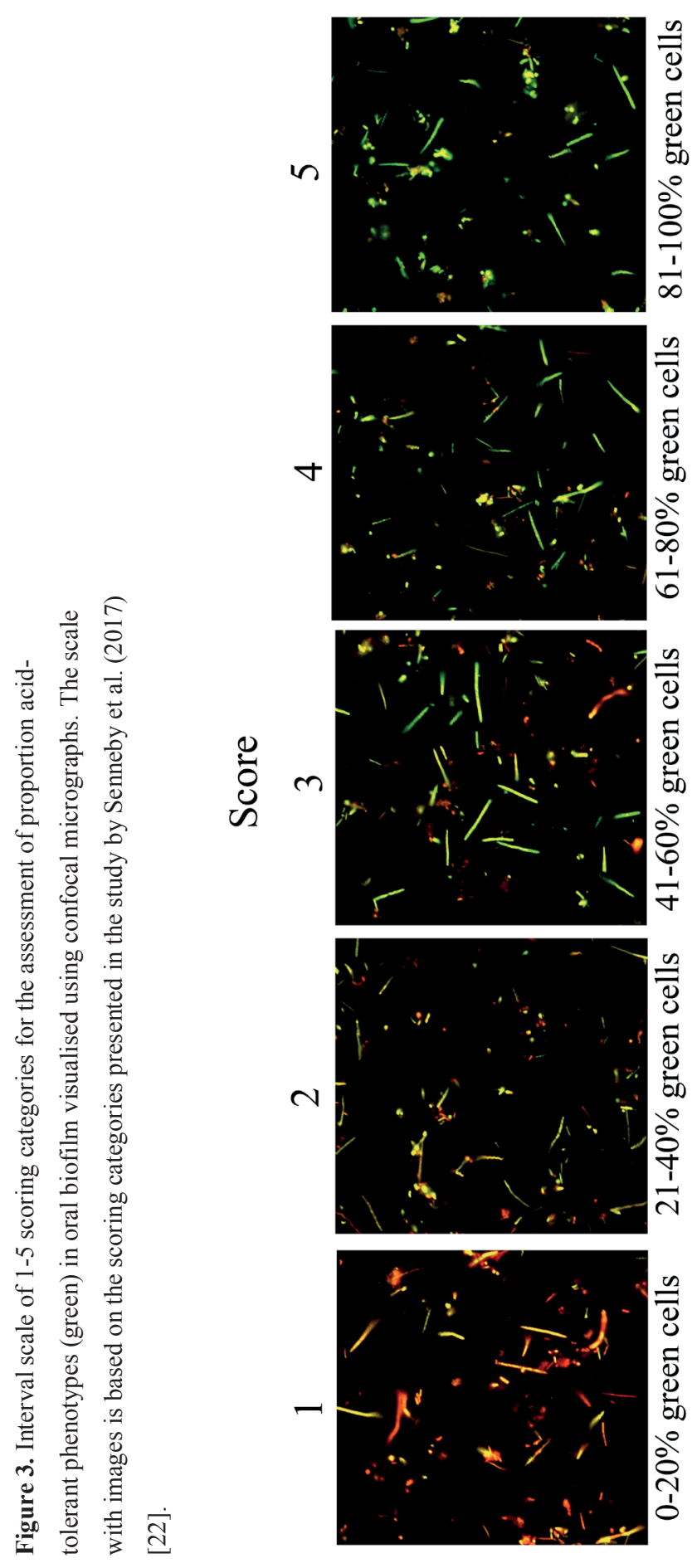




\section{Appendices}

Appendix Table 1. Populated STARD-checklist modified after STARD-statement [16].

Appendix Table 2. Overview of study characteristics.

Appendix Table 3. Overview of interventions in Clinic A and Clinic S during 1-year followup.

Appendix Table 4. Accuracy data of pre-specified thresholds of previous caries experience as index test to identify adolescents with increased risk for developing coronal caries lesions. TP $=$ True Positive $; \mathrm{FP}=$ False Positive $; \mathrm{FN}=$ False Negative $; \mathrm{TN}=$ True Negative $; \mathrm{Se}=$ Sensitivity; $\mathrm{Sp}=$ Specificity PV $=$ Predictive Values; $\mathrm{LR}+=$ Likelihood Ratio of a positive test; LR- = Likelihood Ratio of a negative test.

Appendix Table 5. Accuracy data of pre-specified thresholds of dentists' risk assessments as index test to identify adolescents with increased risk for developing coronal caries lesions. TP $=$ True Positive $; \mathrm{FP}=$ False Positive $; \mathrm{FN}=$ False Negative $; \mathrm{TN}=$ True Negative $; \mathrm{Se}=$ Sensitivity; $\mathrm{Sp}=$ Specificity PV = Predictive Values; LR $+=$ Likelihood Ratio of a positive test; LR- = Likelihood Ratio of a negative test.

Appendix Table 6. Accuracy data of pre-specified thresholds of acid tolerance in dental biofilm as index test to identify adolescents with increased risk for developing coronal caries lesions. $\mathrm{TP}=$ True Positive; FP = False Positive; FN = False Negative; $\mathrm{TN}=$ True Negative; $\mathrm{Se}=$ Sensitivity; $\mathrm{Sp}=$ Specificity PV $=$ Predictive Values; $\mathrm{LR}+=$ Likelihood Ratio of a positive test; LR- = Likelihood Ratio of a negative test. 


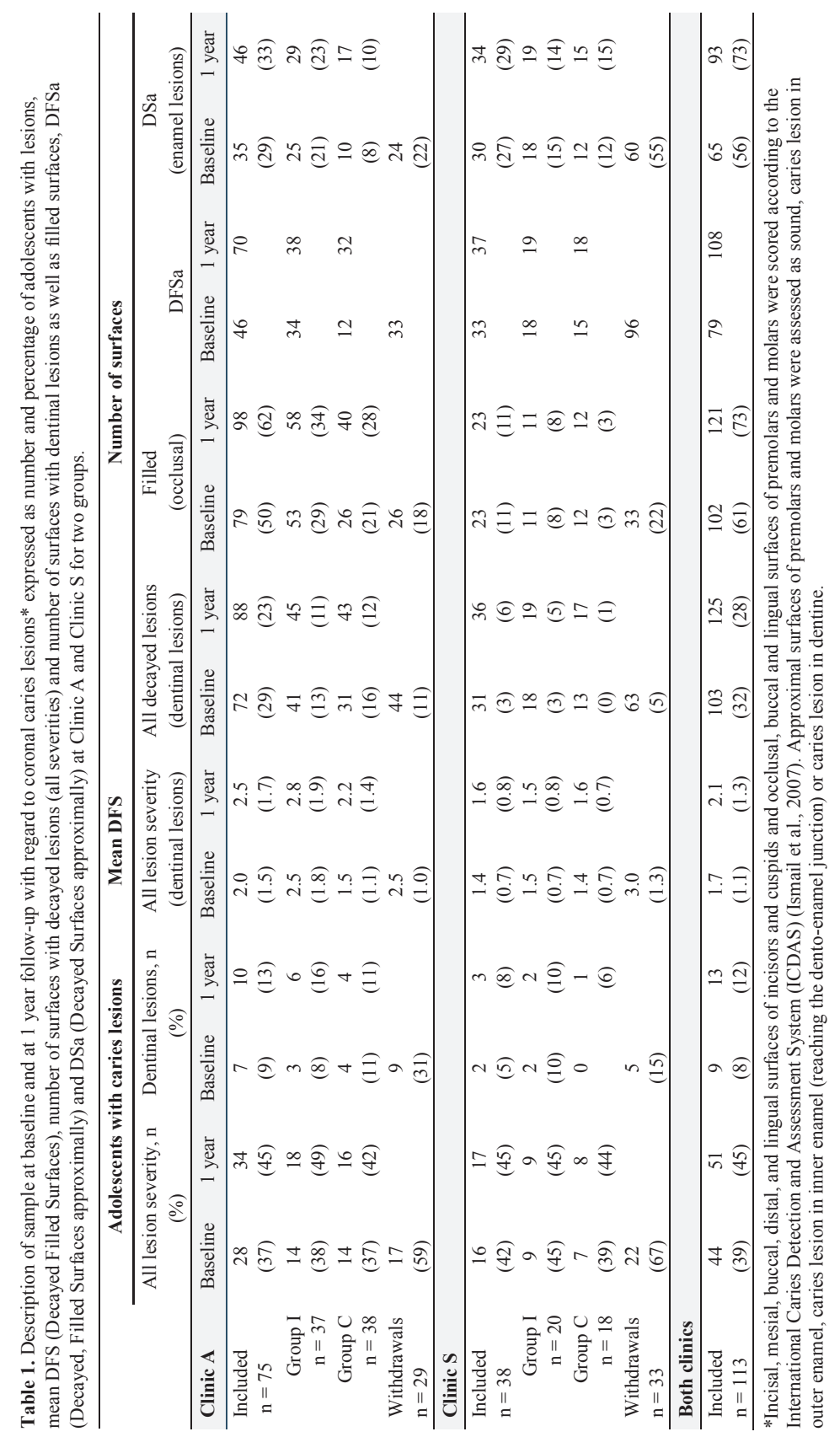




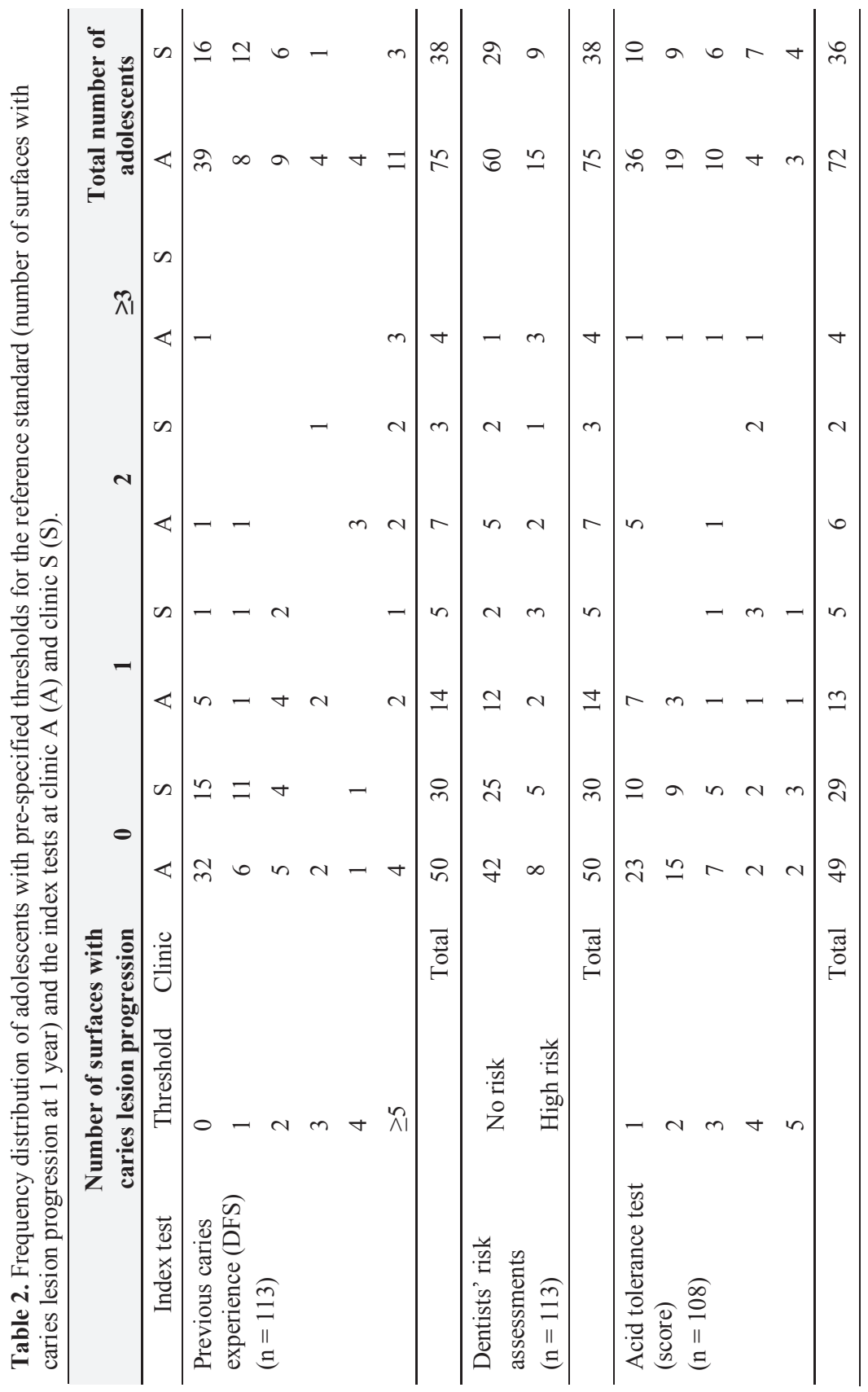




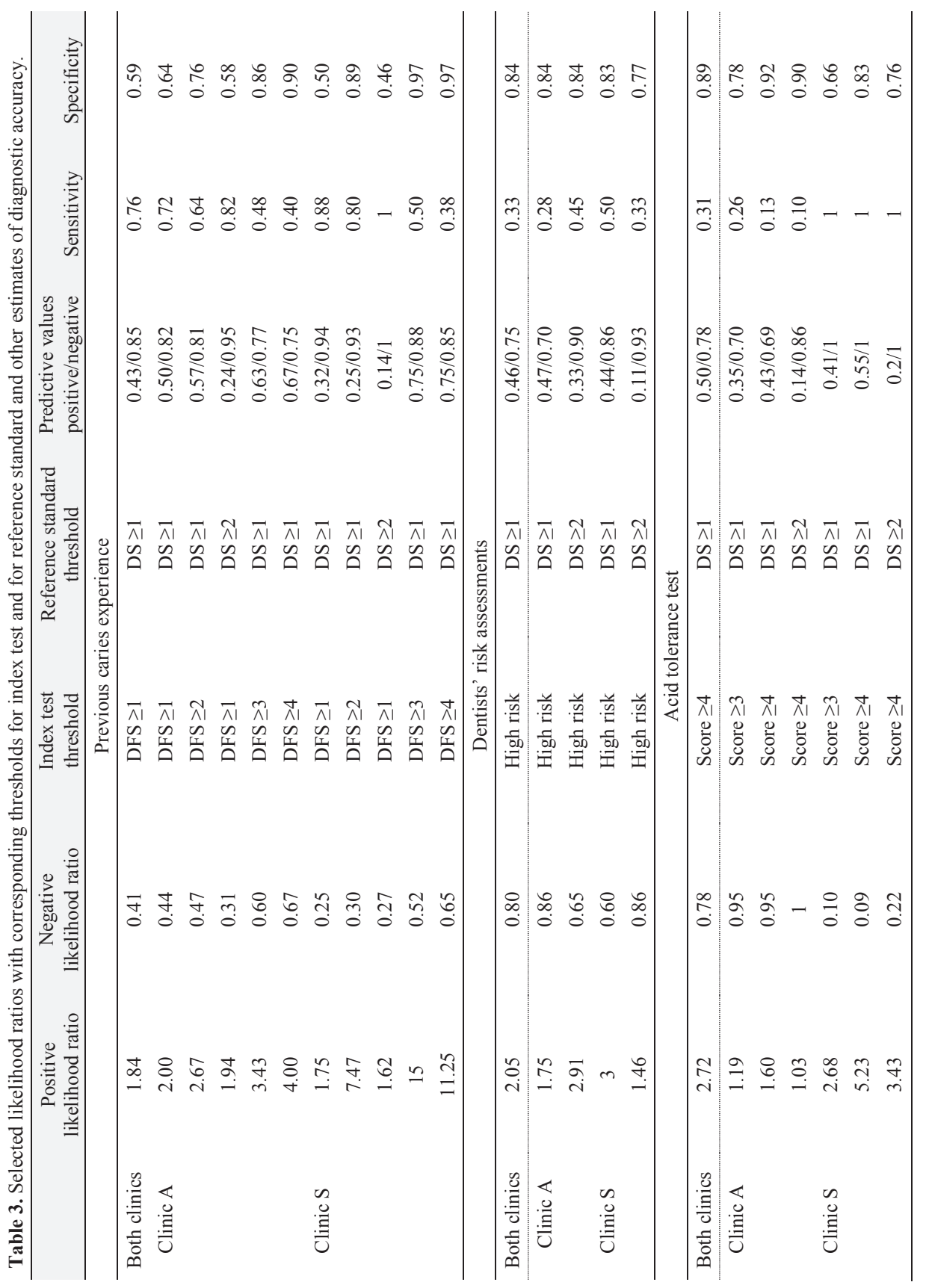


Appendix Table 1. Populated STARD checklist [16].

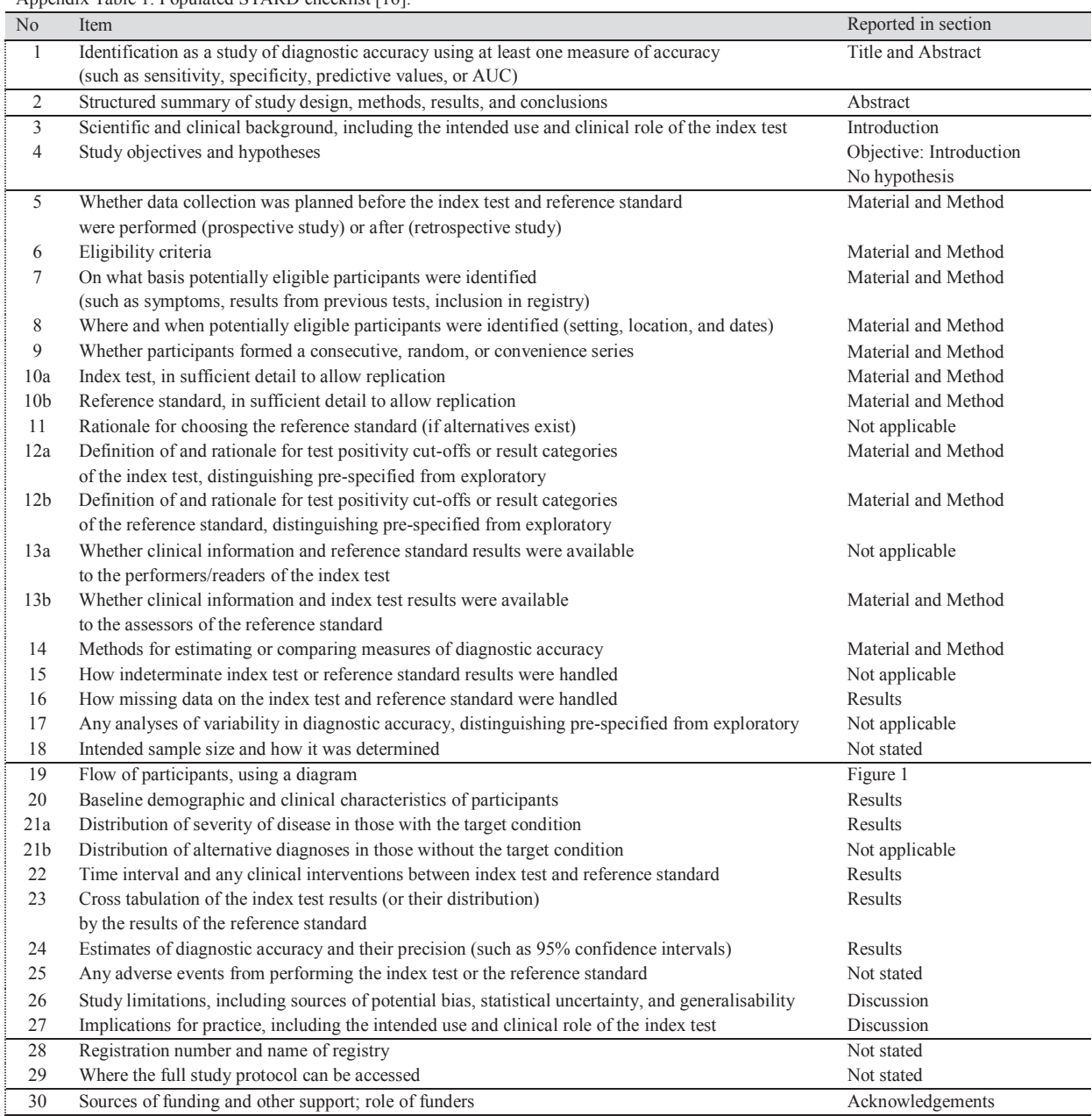




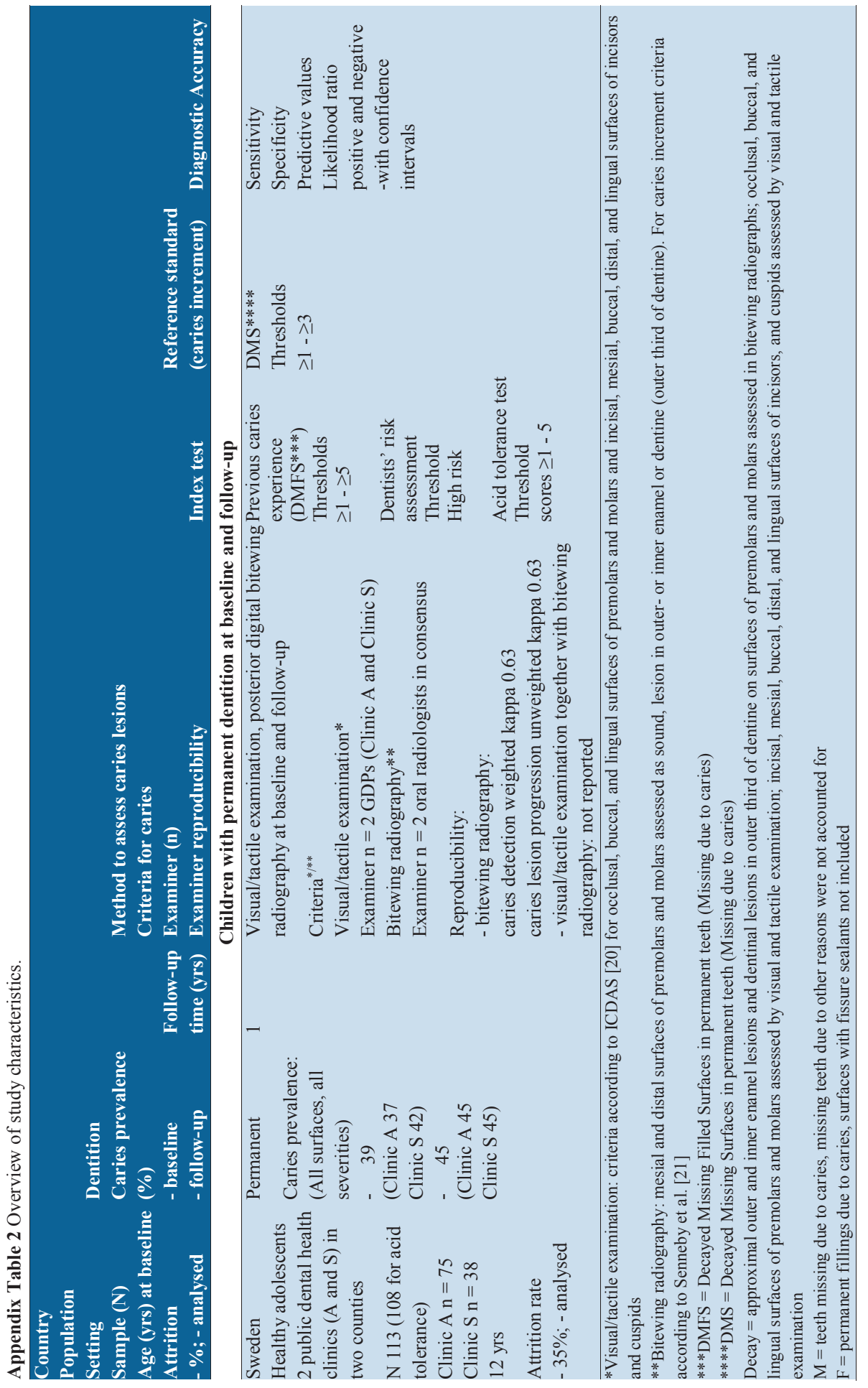




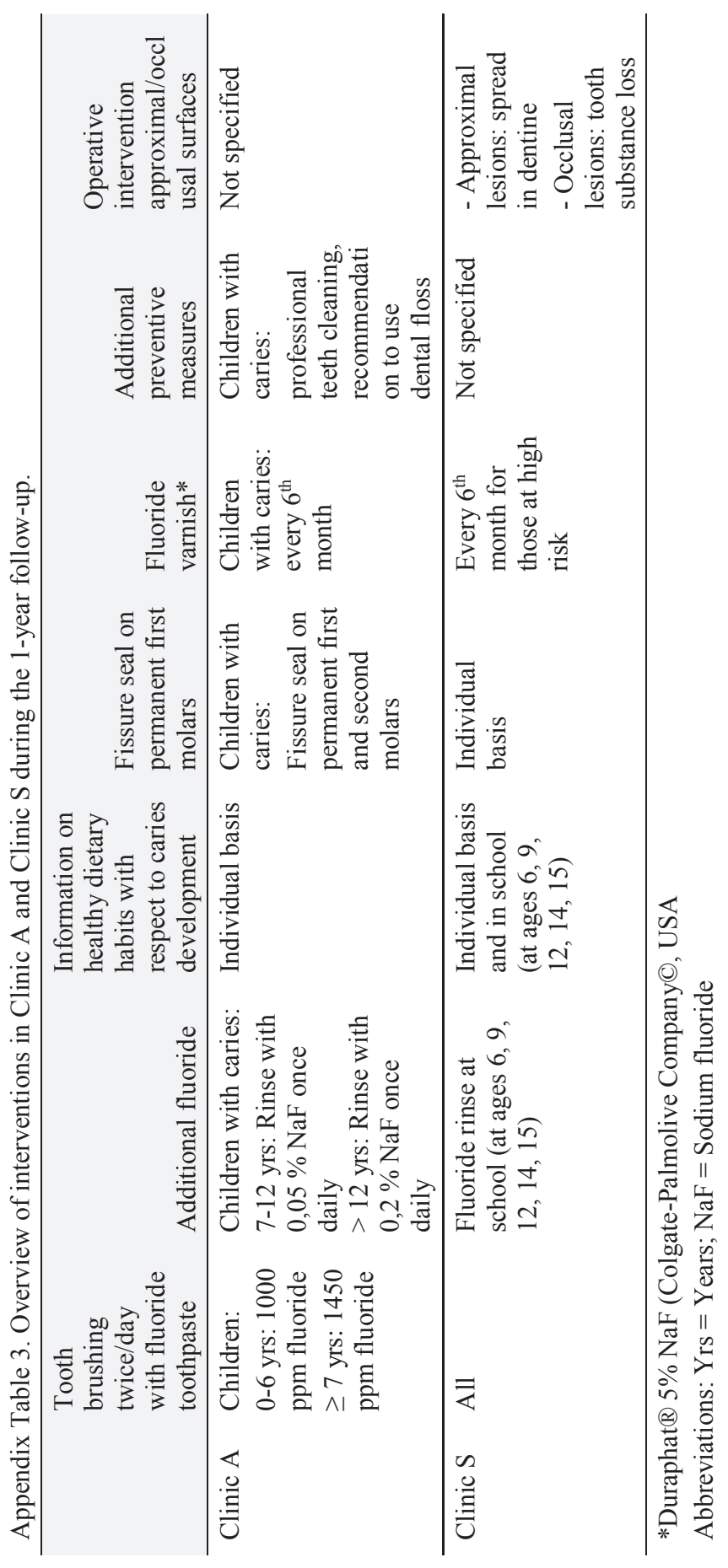




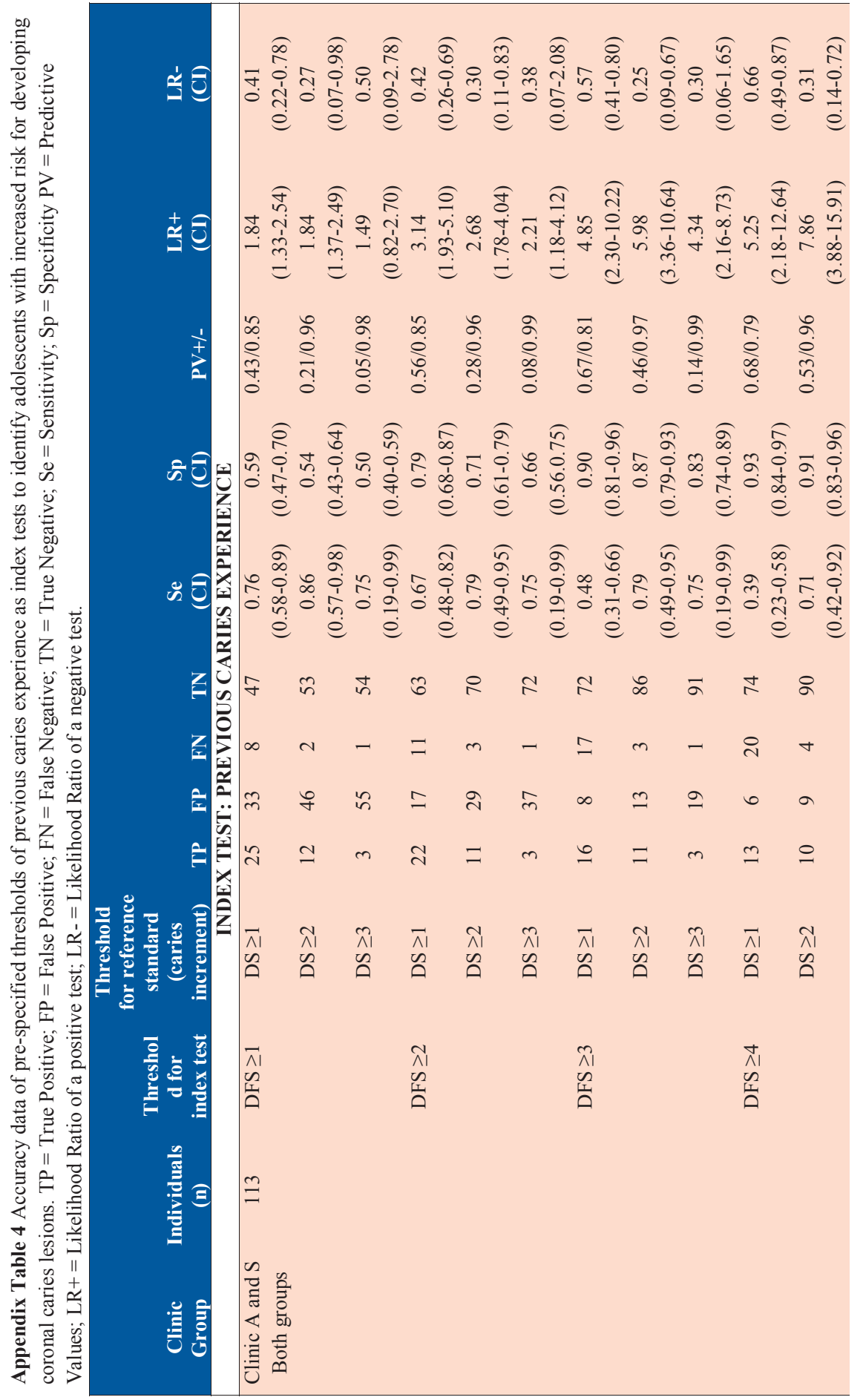




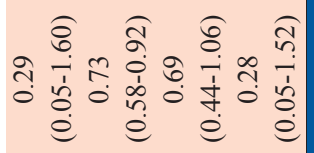

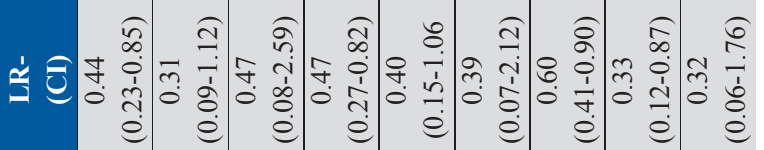

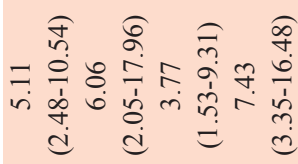

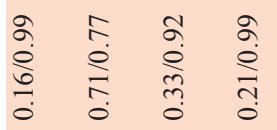

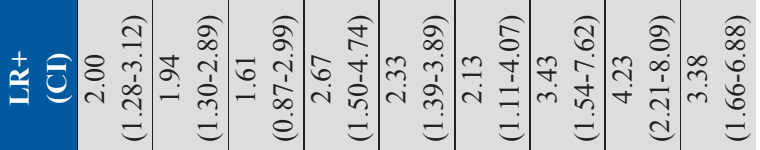

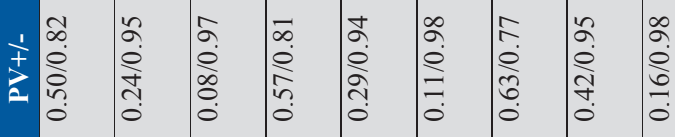

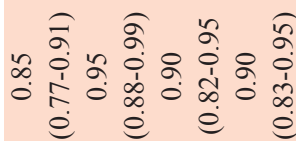

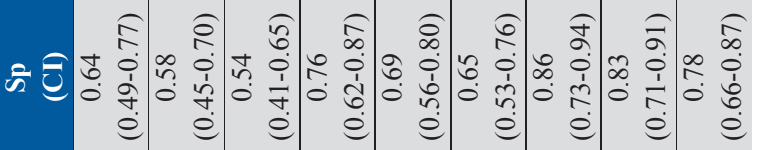

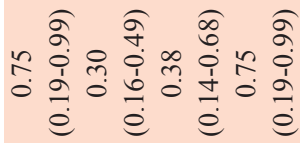

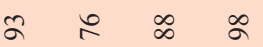

-

$\circ+\circ=$

$m \subseteq n m$

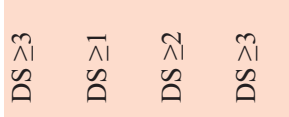

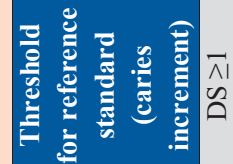

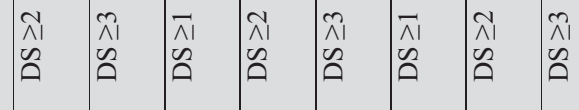

$\overbrace{\substack{n \\ 0}}^{n}$

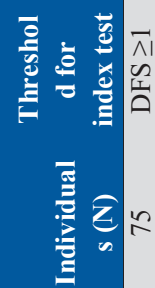

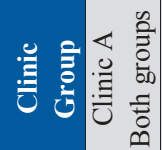




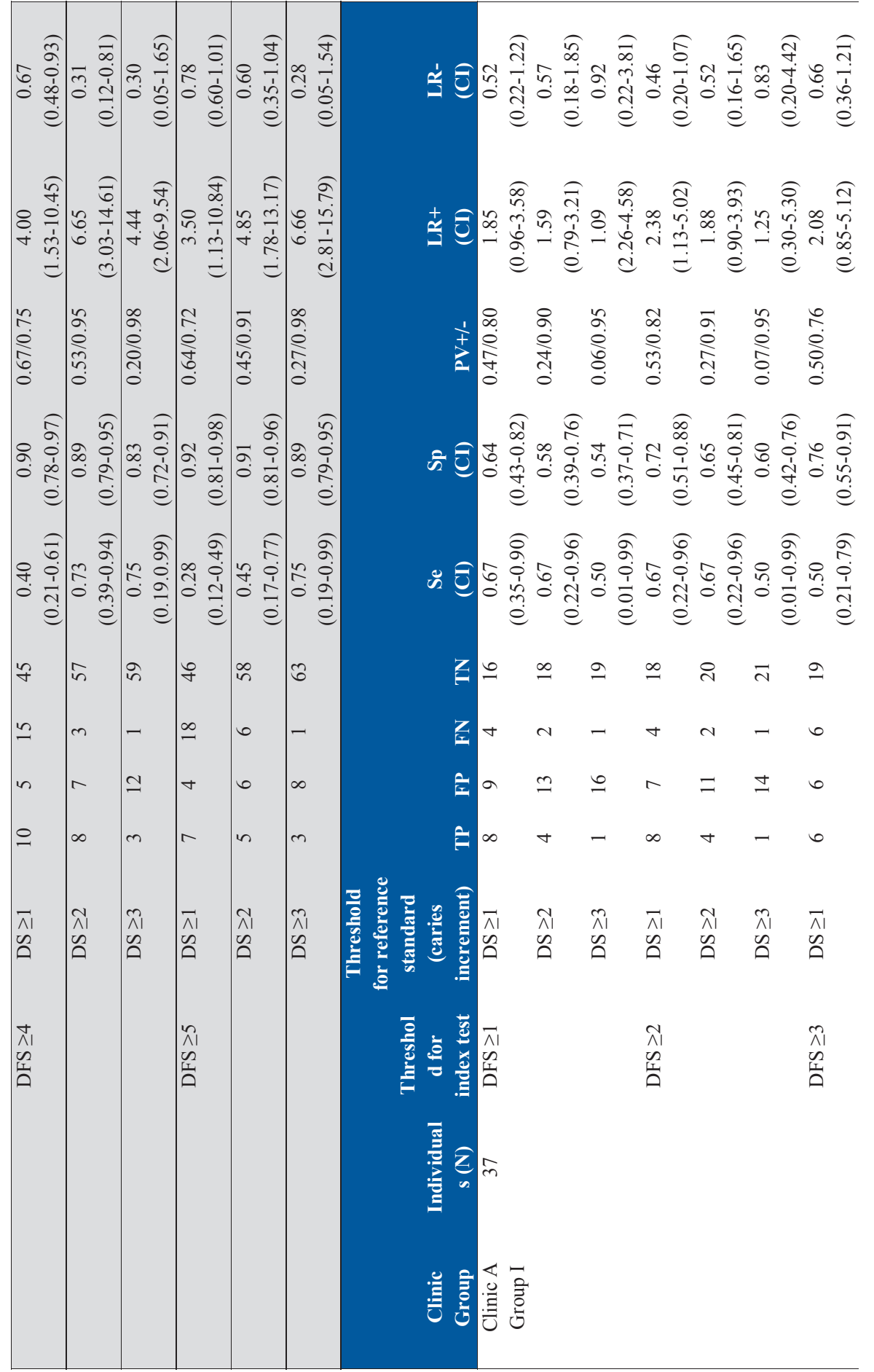




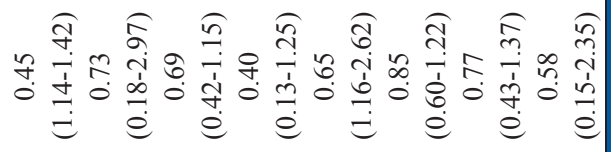

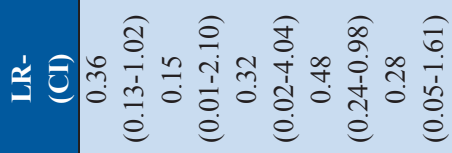

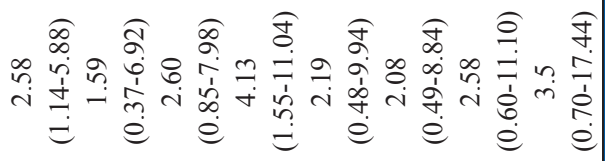

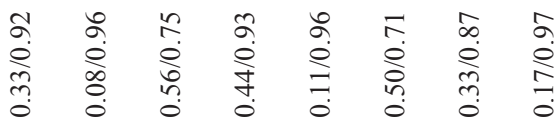

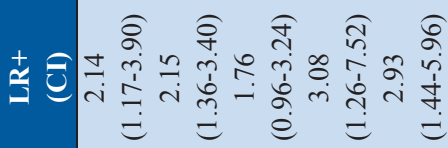

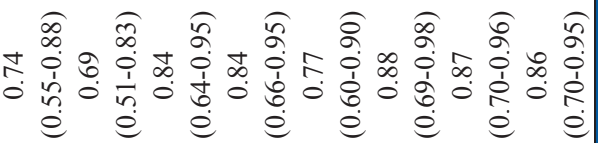

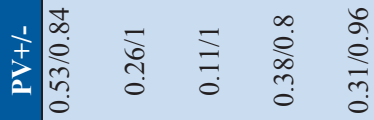

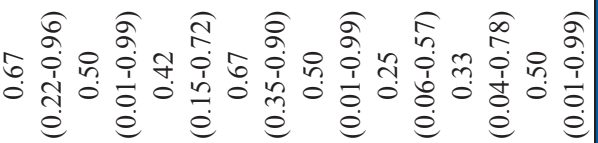

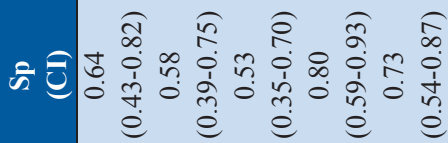

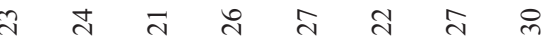

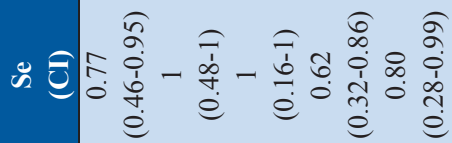

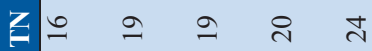

Zn o o n -

教 $\pm \pm n a$

$\hat{F}$ 的 $N$ r

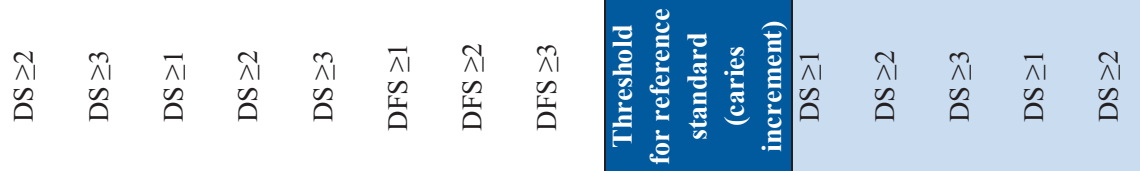

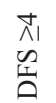

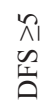

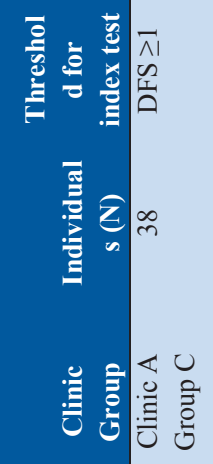




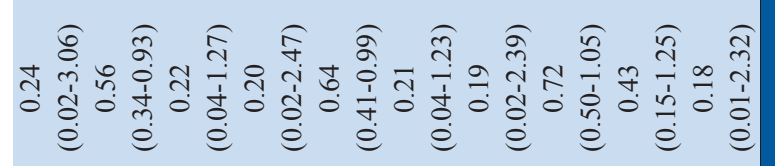

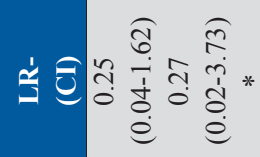

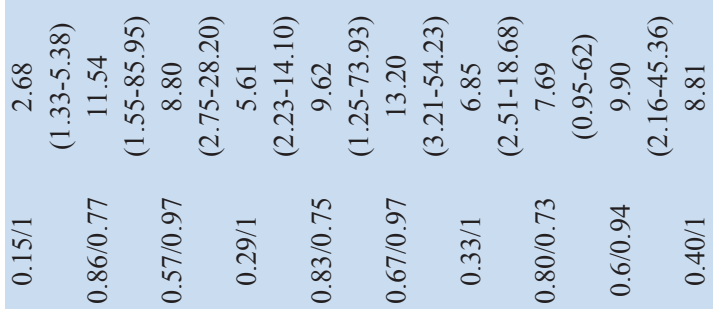

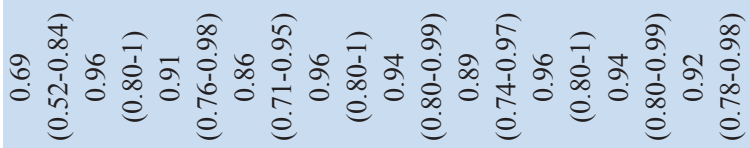

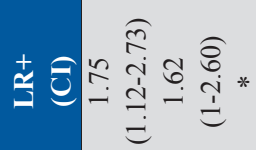

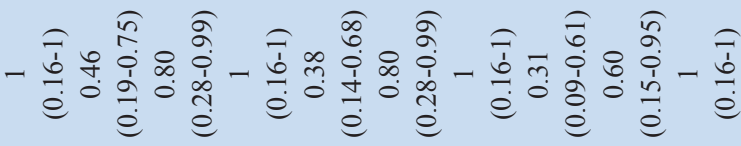

先

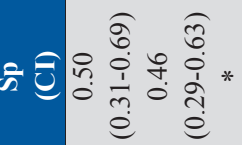

ते ন

$z=0$

ż⿺辶一

ิㅗㄴ

今口m 0

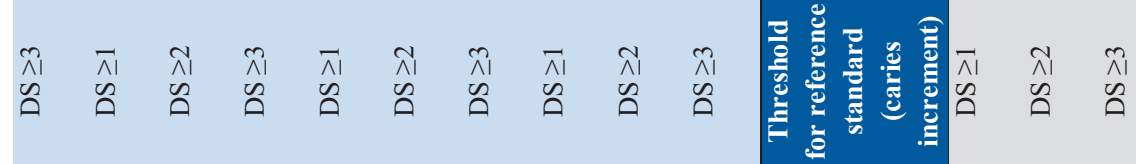
$\hat{\lambda}$
$\hat{n}$
全

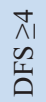

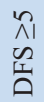

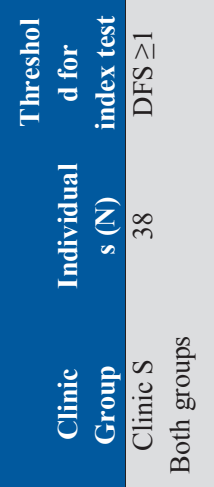




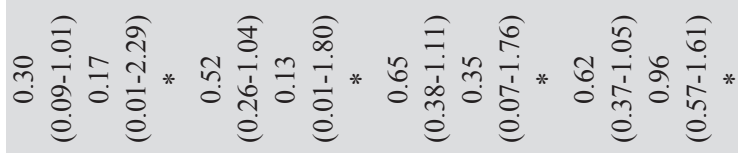

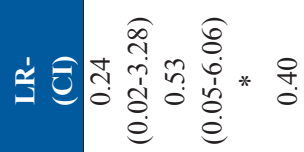

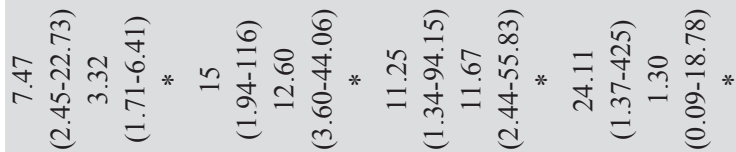

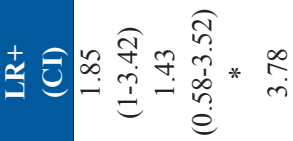

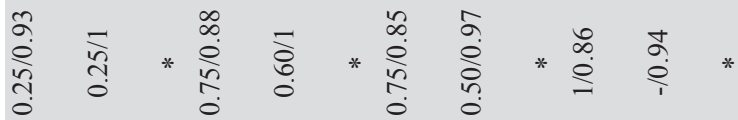

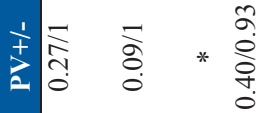

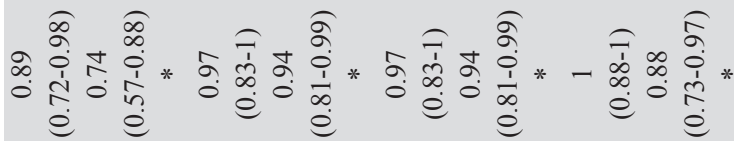

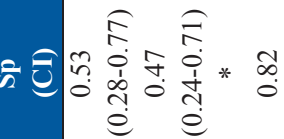

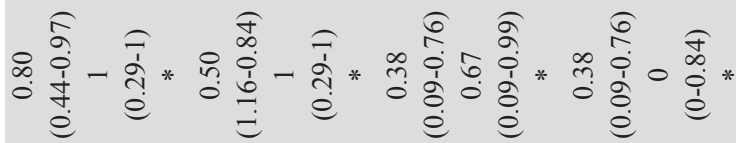

मं

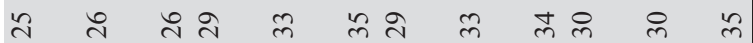

Za $a$ a

$z \circ 00-$

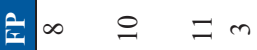

n a $\simeq-4 m-n+0+m$

Aิm- ON

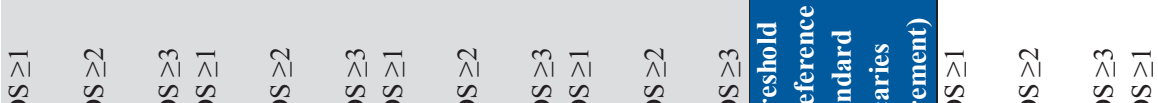
นิ นิ นิน

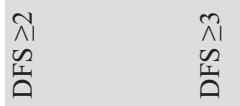

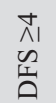

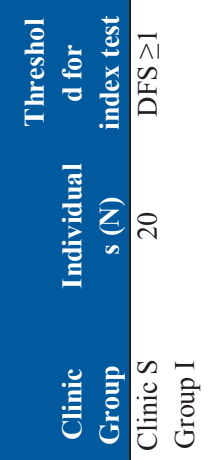




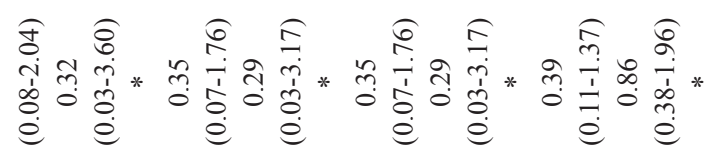

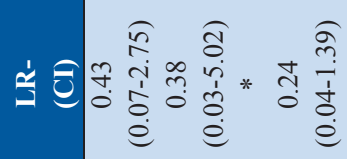

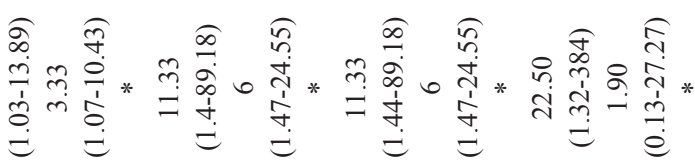

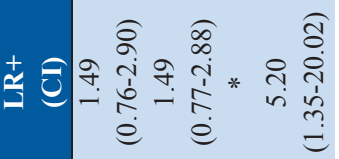

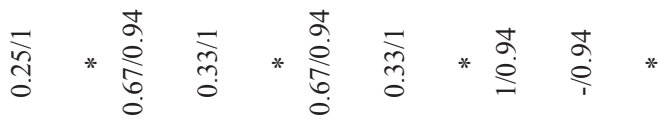

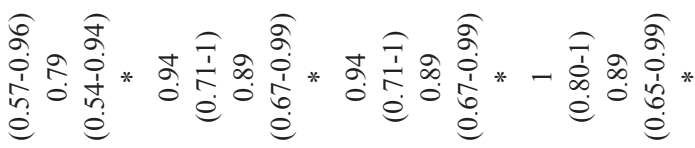

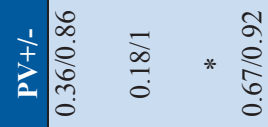

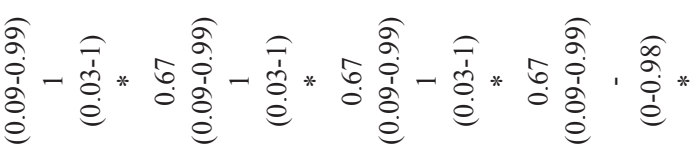

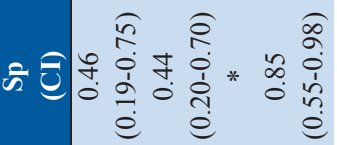

$\because \because \underline{I} \simeq \simeq \simeq \subseteq$

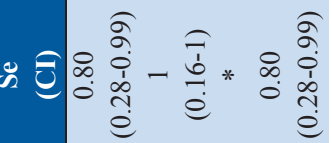

o o- 0 o- 0 o-

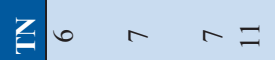

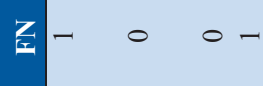

a n-a m-a mo a a

- on - on t on o o

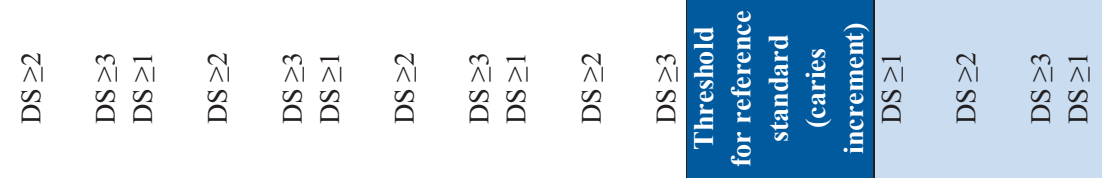

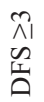

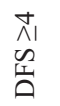

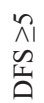

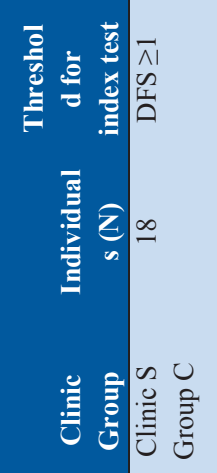

